University of Tennessee Health Science Center

UTHSC Digital Commons

$12-2013$

\title{
Development and Characterization of a Recombinant Vesicular Stomatitis Virus (rVSV) for the Treatment of Glioblastoma
}

\author{
Erika A. Dillard \\ University of Tennessee Health Science Center
}

Follow this and additional works at: https://dc.uthsc.edu/dissertations

Part of the Neoplasms Commons, and the Viruses Commons

\section{Recommended Citation}

Dillard, Erika A. , "Development and Characterization of a Recombinant Vesicular Stomatitis Virus (rVSV) for the Treatment of Glioblastoma" (2013). Theses and Dissertations (ETD). Paper 66. http://dx.doi.org/ 10.21007/etd.cghs.2013.0070.

This Dissertation is brought to you for free and open access by the College of Graduate Health Sciences at UTHSC Digital Commons. It has been accepted for inclusion in Theses and Dissertations (ETD) by an authorized administrator of UTHSC Digital Commons. For more information, please contact jwelch30@uthsc.edu. 


\title{
Development and Characterization of a Recombinant Vesicular Stomatitis Virus (rVSV) for the Treatment of Glioblastoma
}

\author{
Abstract \\ Background: Over the past 30 years, little has changed in the treatment modalities and prognosis of \\ patients suffering from Glioblastoma multiforme (GBM), the most common and by far the most \\ devastating adult primary malignant brain tumor. Conventional therapies provide only a marginal increase \\ in survival of GBM patients, post-diagnosis. Therefore, more novel means of treating GBM are needed to \\ increase long-term survival and quality of life for those affected. Replication competent oncolytic viruses \\ (OVs) have recently emerged as a possible option for treatment of high-grade gliomas. Particularly, \\ recombinant vesicular stomatitis virus (rVSV), an enveloped, negative strand RNA virus, has shown \\ promising results in preclinical studies. Tumor selectivity of VSV is thought to be associated with tumor \\ specific defects in the interferon (IFN) pathway. However, largely due to insufficient attention on the role \\ the immune system plays in efficacy of treatment, potential OVs have been obstructed from moving \\ through the clinical trial pipeline past Phase I/II studies. INCP12.1 is a novel recombinant VSV vector \\ possessing specific mutations in the matrix protein. Thes mutations have been shown to promote viral \\ attenuation in normal cells while maintaining cytotoxicity in a number of tumor cell lines. We aim to \\ characterize and further develop this novel agent for the treatment of GBM. Methods: In order to \\ determine differences between rNCP12.1 and wtVSV and to determine specificity of rNCP12.1 for tumor \\ over normal cells, cell rounding assays, one-step growth curves, and cytotoxicity assays were performed \\ in normal glial and tumor glial cell lines. To understand the basis of this selectivity and whether it \\ correlated with the antiviral responses of IFN, expression levels of IFN and IFN stimulated genes (ISGs) \\ were quantified, production of active IFN was measured, and the ability of cells to inhibit viral infection in \\ response to exogenous IFN was determined. In vivo experiments were designed and carried out to test \\ for oncolytic activity of rNCP12.1 in immunocompetent animal models of intracranial glioma. A single \\ injection of rNCP12.1 was administered into previously implanted F98-GFP tumors. Tumor load and \\ parameters of morbidity were assessed at 15 days following tumor implantation and long term at the \\ time of euthanasia. Viral induced immune responses were assessed by the IFN bioassay and detection of \\ circulating anti-VSV antibodies were achieved by Western blot analysis and a neutralizing antibody assay. \\ Experimental methods of virus administration for treatment of glioma were further tested including \\ multiple injections, injections using different VSV serotypes, continuous infusion of virus using \\ implantable osmotic pumps, and pre-infected autologous carrier cells. These methods were designed to \\ enhance anti-tumor effect by managing the negative effects of the tumor microenvironment and of a \\ functional immune system on viral therapy. Results and Conclusion: rNCP12.1 was shown to be an \\ attenuated strain of VSV that has clear differences in its growth and induction of the IFN response \\ pathway in normal cells. It has a preference for growth in tumor cells as determined by viral titers, cell \\ rounding, and cell viability post infection. This preference varied based on cellular expression of a \\ particular IFN phenotype. The importance of this molecular versus histological cell profile was evident \\ even in the performance of rNCP12.1 on human glioma cell lines that differ in their expression of IFN. In \\ vivo evaluation of rNCP12.1 against a highly IFN resistant rat glioma cell line, F98, demonstrated its ability \\ to decrease tumor size while eliciting a peripheral response to virus that protects normal tissue but also \\ shortens its therapeutic window and the ability to sustain reduction of tumor over time. Several \\ experimental methods in delivery of virus proved to be beneficial, including administering an additional \\ dose of virus using a different serotype to bypass antiviral neutralizing responses and by shielding virus \\ from the immune system through the use of tumor carrier cells. As an additional benefit, the latter was \\ shown to have a unique pattern in eliciting tumor specific antibodies that was different from those \\ increased by therapy with virus alone. This method also increased recovery of virus from brain tissue even \\ after 20 days post treatment.
}


Our data supports the capability of rVSV vectors as treatment for GBM. Specifically rNCP12.1 therapy increased survival while decreasing tumor load, depending on method of administration. When given alone, virus is ultimately immunogenic and prompts anti-viral as well as anti-tumor immune responses. However, when shielded from the immune system, antiviral responses are minimal while anti-tumor responses are sustained. To this end, therapy cannot be fully addressed without addressing the effects the immune system has on therapy and on the host. Future studies, should include not only evaluation of tumor load, morbidity, and side effects of viral therapy but also immune responses especially those that are likely to enhance therapy past the acute stages of disease.

\section{Document Type}

Dissertation

Degree Name

Doctor of Philosophy (PhD)

\section{Program}

Biomedical Sciences

\section{Research Advisor}

Michael A. Whitt, Ph.D.

\section{Keywords}

Glioblastoma Multiforme, Interferon, Matrix Protein, Oncolytic Virus, Vesicular Stomatitis Virus.

\section{Subject Categories}

Diseases | Medicine and Health Sciences | Neoplasms | Viruses 
Development and Characterization of a Recombinant Vesicular Stomatitis Virus

(rVSV) for the Treatment of Glioblastoma

\author{
A Dissertation \\ Presented for \\ The Graduate Studies Council \\ The University of Tennessee \\ Health Science Center
}

\begin{abstract}
In Partial Fulfillment
Of the Requirements for the Degree

Doctor of Philosophy

From The University of Tennessee
\end{abstract}

By

Erika A. Dillard

December 2013 
Copyright (C) 2013 by Erika A. Dillard. All rights reserved. 


\section{DEDICATION}

This dissertation is dedicated to my mother, Rita Rooks Johnson, and grandmother, Bessie Rooks, two of the most influential people in my life who have been perfect examples of selflessness and courage for me throughout the years. Their encouraging words will always be a source of motivation for me now and always. 


\section{ACKNOWLEDGEMENTS}

I would first like to thank God for blessing me with the responsibility to learn the basic and clinical science of human diseases in order to help others. I would like to thank my mentor, Michael A. Whitt, Ph.D., for my graduate training and for affording me the opportunity to work in his lab. His guidance and support has been invaluable. I would also like to thank Dr. Christopher Duntsch for sharing with me his experience in neurooncology and sparking in me the interest for a career in neurosurgery and neurooncological research. Many thanks to my committee members Suzanne Baker, Ph.D., Lawrence Pfeffer, Ph.D., Tayebeh Pourmotabbed, Ph.D., and Charles Russell, Ph.D. for their direction and counsel during my graduate career. I would like to specifically thank Drs. Lawrence Pfeffer and Tayebeh Pourmotabbed for opening their labs to help me master techniques that have been crucial to my project. I would like to thank the members of the Whitt lab, past and present, including Carolyn Matthews, Elizabeth "Lane" Matheny, Ph.D., Himangi Jayakar, Ph.D., and Chad Mire, Ph.D. for their support over the course of my graduate experience, both in and out of the lab. Additionally, I want to thank all of the members of the Molecular Sciences Building, especially to my $6^{\text {th }}$ floor mates. Thanks is due to the members of the former Neurosurgery lab, including Xiofei Wang, Qihong Zhou, M.D., Raya Krutilina Ph.D., Mariya Lane, PharmD, and Zoran Pavicevic, M.D., for being a wonderful resource to me throughout my project.

I must acknowledge the other M.D./Ph.D. students, though few in number, who have been wonderful role models and peer mentors to me over the years. Your guidance and support, throughout this experience have been greatly appreciated. I want to give a special thanks to the administration and staff of the Colleges of Graduate Health Sciences and Medicine including Robert Shreve, Ph.D., Pat Ryan, Ph.D., Susan Brewer, M.D., Elise Moore, Ruby Bland, and the late Janie Van Prooijen for helping me complete this journey.

Last but not least, I want to give a special thanks to the University of Tennessee Health Science Center for its financial support through the Clinical and Translational Science Institute T32 funding and Bridge funding. 


\begin{abstract}
Background: Over the past 30 years, little has changed in the treatment modalities and prognosis of patients suffering from Glioblastoma multiforme (GBM), the most common and by far the most devastating adult primary malignant brain tumor. Conventional therapies provide only a marginal increase in survival of GBM patients, post-diagnosis. Therefore, more novel means of treating GBM are needed to increase long-term survival and quality of life for those affected. Replication competent oncolytic viruses (OVs) have recently emerged as a possible option for treatment of high-grade gliomas. Particularly, recombinant vesicular stomatitis virus (rVSV), an enveloped, negative strand RNA virus, has shown promising results in preclinical studies. Tumor selectivity of VSV is thought to be associated with tumor specific defects in the interferon (IFN) pathway. However, largely due to insufficient attention on the role the immune system plays in efficacy of treatment, potential OVs have been obstructed from moving through the clinical trial pipeline past Phase I/II studies. rNCP12.1 is a novel recombinant VSV vector possessing specific mutations in the matrix protein. Thes mutations have been shown to promote viral attenuation in normal cells while maintaining cytotoxicity in a number of tumor cell lines. We aim to characterize and further develop this novel agent for the treatment of GBM.
\end{abstract}

Methods: In order to determine differences between rNCP12.1 and wtVSV and to determine specificity of rNCP12.1 for tumor over normal cells, cell rounding assays, one-step growth curves, and cytotoxicity assays were performed in normal glial and tumor glial cell lines. To understand the basis of this selectivity and whether it correlated with the antiviral responses of IFN, expression levels of IFN and IFN stimulated genes (ISGs) were quantified, production of active IFN was measured, and the ability of cells to inhibit viral infection in response to exogenous IFN was determined. In vivo experiments were designed and carried out to test for oncolytic activity of rNCP12.1 in immunocompetent animal models of intracranial glioma. A single injection of rNCP12.1 was administered into previously implanted F98-GFP tumors. Tumor load and parameters of morbidity were assessed at 15 days following tumor implantation and long term at the time of euthanasia. Viral induced immune responses were assessed by the IFN bioassay and detection of circulating anti-VSV antibodies were achieved by Western blot analysis and a neutralizing antibody assay. Experimental methods of virus administration for treatment of glioma were further tested including multiple injections, injections using different VSV serotypes, continuous infusion of virus using implantable osmotic pumps, and pre-infected autologous carrier cells. These methods were designed to enhance anti-tumor effect by managing the negative effects of the tumor microenvironment and of a functional immune system on viral therapy.

Results and Conclusion: rNCP12.1 was shown to be an attenuated strain of VSV that has clear differences in its growth and induction of the IFN response pathway in normal cells. It has a preference for growth in tumor cells as determined by viral titers, cell rounding, and cell viability post infection. This preference varied based on cellular expression of a particular IFN phenotype. The importance of this molecular versus 
histological cell profile was evident even in the performance of rNCP12.1 on human glioma cell lines that differ in their expression of IFN.

In vivo evaluation of rNCP12.1 against a highly IFN resistant rat glioma cell line, F98, demonstrated its ability to decrease tumor size while eliciting a peripheral response to virus that protects normal tissue but also shortens its therapeutic window and the ability to sustain reduction of tumor over time. Several experimental methods in delivery of virus proved to be beneficial, including administering an additional dose of virus using a different serotype to bypass antiviral neutralizing responses and by shielding virus from the immune system through the use of tumor carrier cells. As an additional benefit, the latter was shown to have a unique pattern in eliciting tumor specific antibodies that was different from those increased by therapy with virus alone. This method also increased recovery of virus from brain tissue even after 20 days post treatment.

Our data supports the capability of rVSV vectors as treatment for GBM. Specifically rNCP12.1 therapy increased survival while decreasing tumor load, depending on method of administration. When given alone, virus is ultimately immunogenic and prompts anti-viral as well as anti-tumor immune responses. However, when shielded from the immune system, anti-viral responses are minimal while antitumor responses are sustained. To this end, therapy cannot be fully addressed without addressing the effects the immune system has on therapy and on the host. Future studies, should include not only evaluation of tumor load, morbidity, and side effects of viral therapy but also immune responses especially those that are likely to enhance therapy past the acute stages of disease. 


\section{TABLE OF CONTENTS}

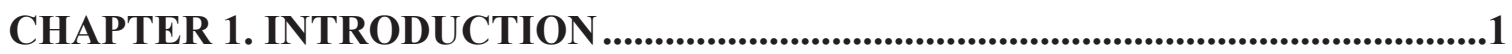

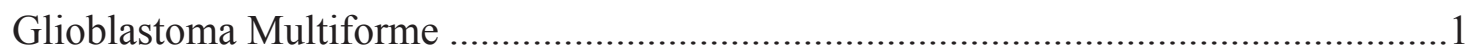

Definition and Classification .......................................................................

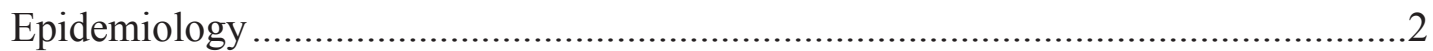

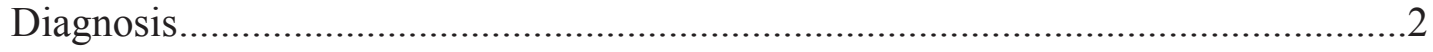

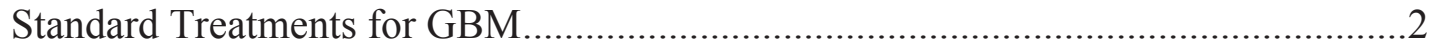

Molecular Subtypes of Glioblastoma................................................................. 5

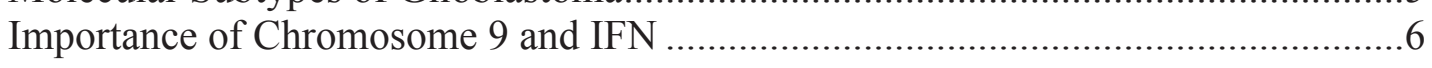

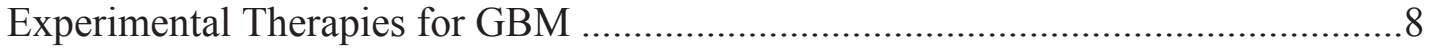

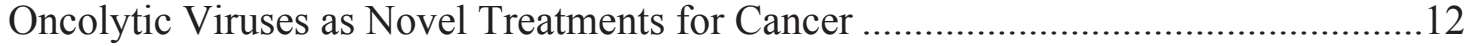

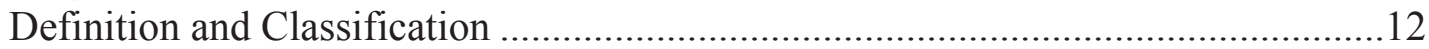

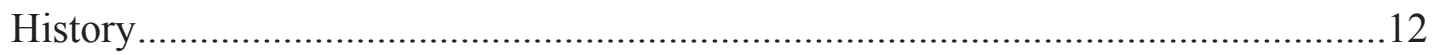

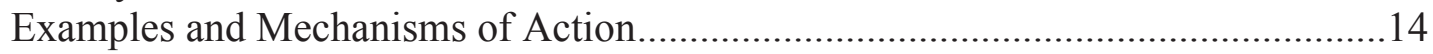

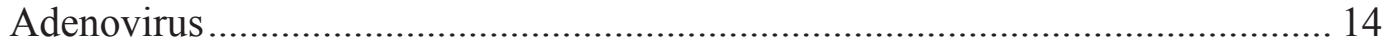

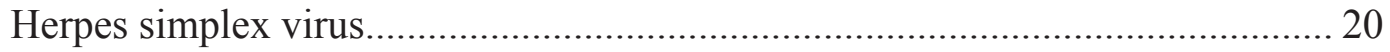

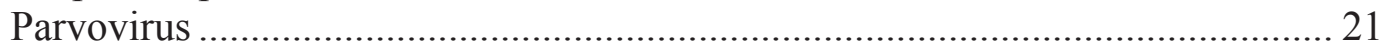

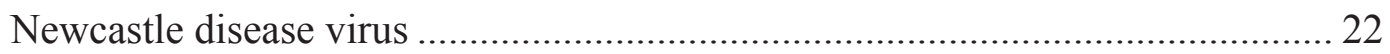

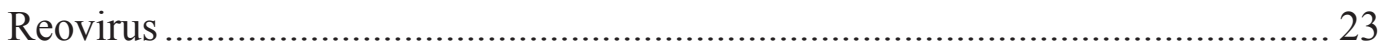

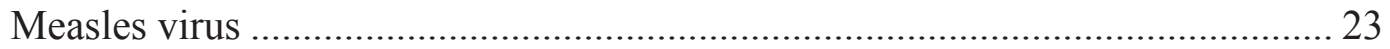

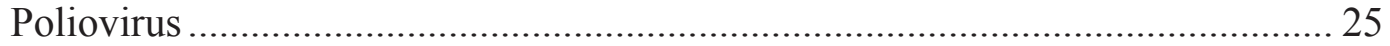

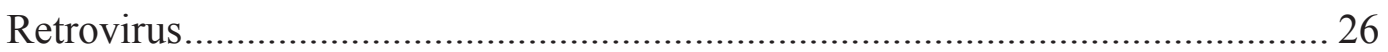

Future of Oncolytic Therapy: Challenges to Overcome ......................................27

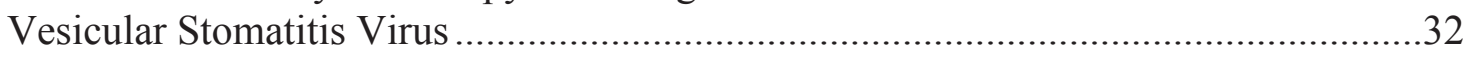

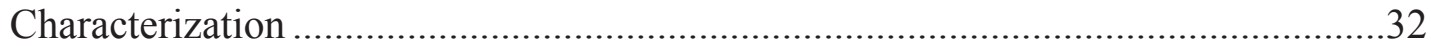

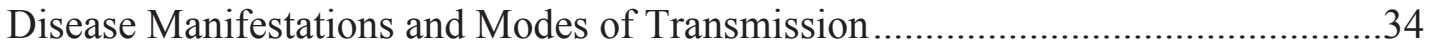

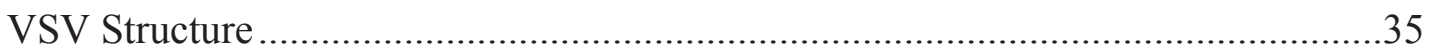

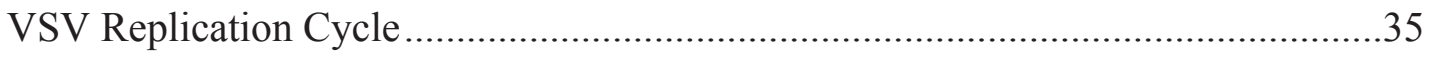

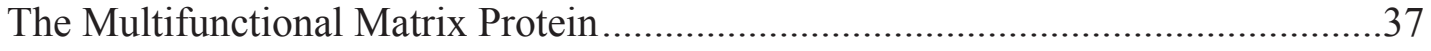

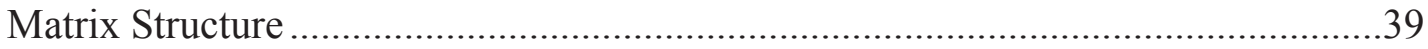

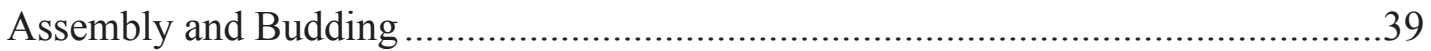

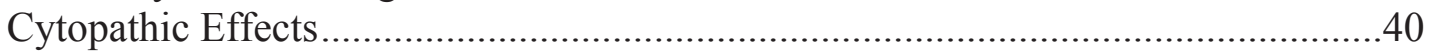

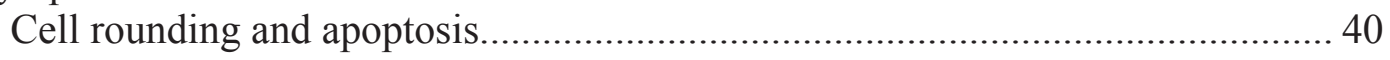

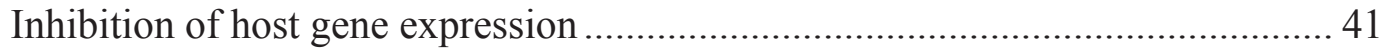

Inhibition of transcription and translation ................................................ 41

Inhibition of nucleocytoplasmic transport (NCT) .................................. 43

Matrix domains important for CPE............................................................. 43

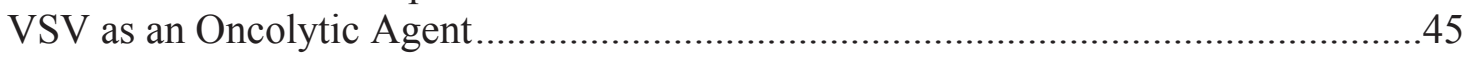

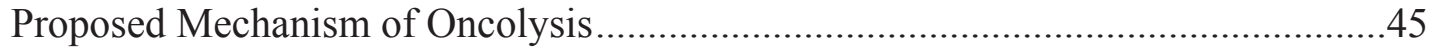

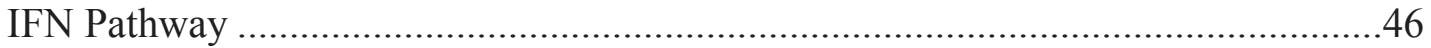

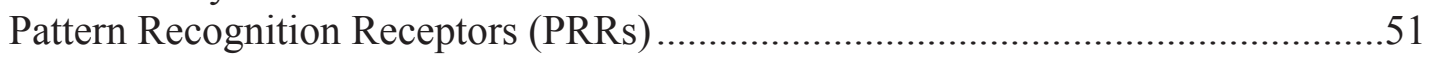

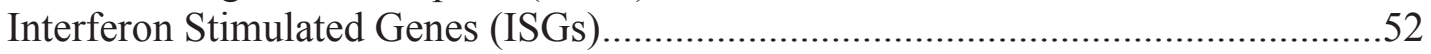

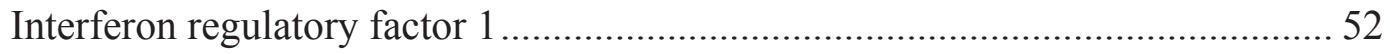

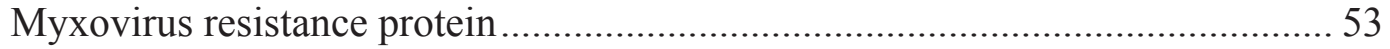




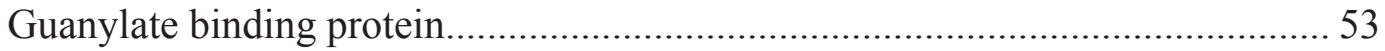

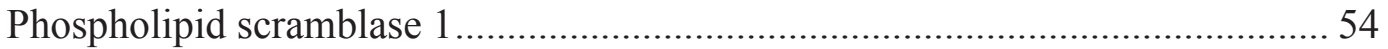

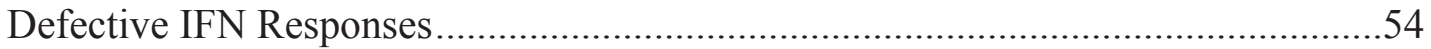

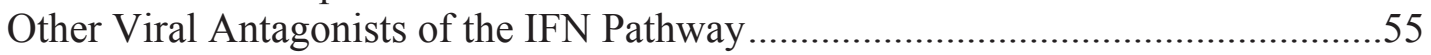

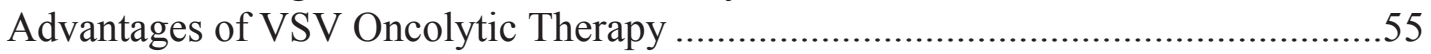

Disadvantages of VSV Oncolytic Therapy ………………...................................56

Examples of VSV-Derived OVs in Preclinical and Clinical Trials ............................57

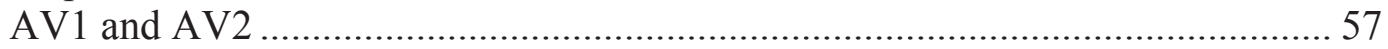

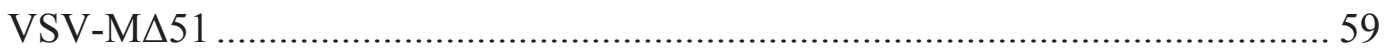

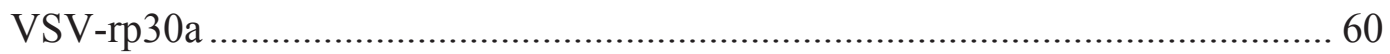

VSV-IFN $\beta$ (mIFN $\beta$, mouse IFN $\beta$; rIFN $\beta$, rat IFN $\beta$; hIFN $\beta$, human IFN $\beta)$....... 60

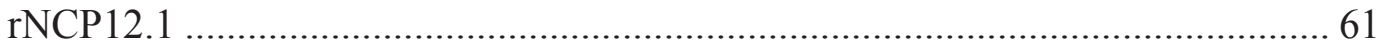

CHAPTER 2. MATERIALS AND METHODS.............................................................65

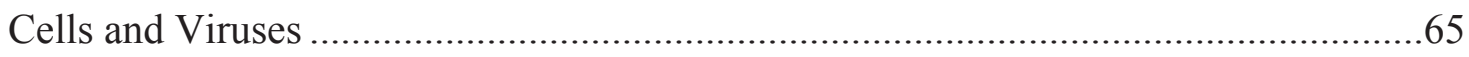

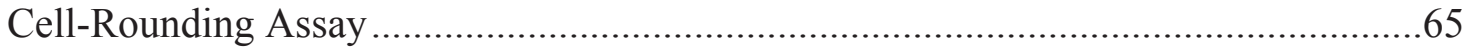

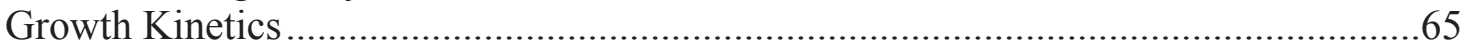

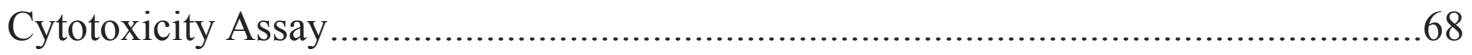

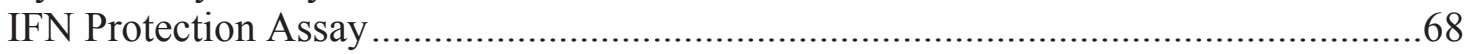

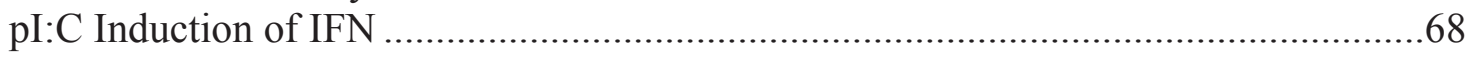

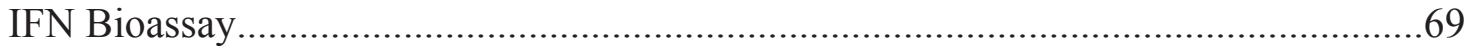

Viral Infection for IFN Bioassay and ISG qRT-PCR ………...................................69

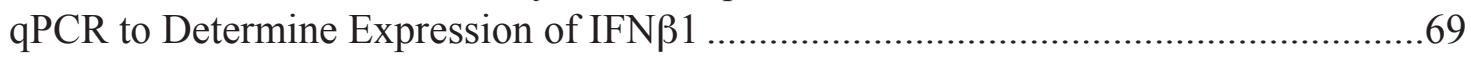

Quantitative RT-PCR (qRT-PCR) to Determine Expression of IFN $\beta 1$ and ISGs ........72

Western Blot Detection of Neutralizing Antibodies......................................................72

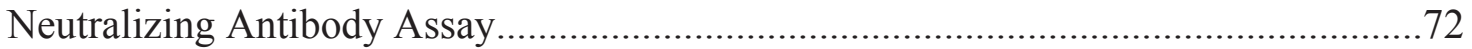

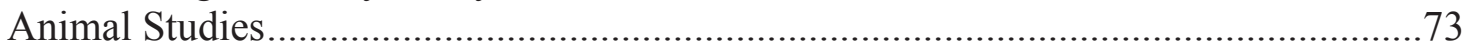

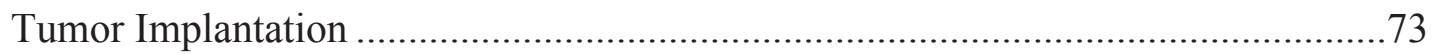

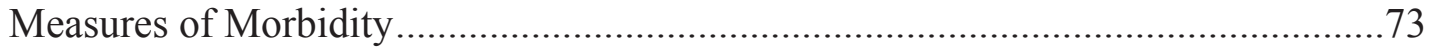

Cerebrospinal Fluid (CSF) Collection .................................................................76

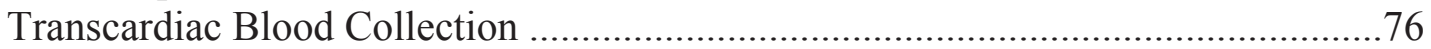

Preparation of Concentrated Virus for Intracranial Injections ....................................76

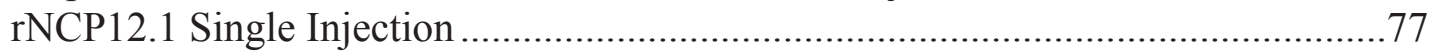

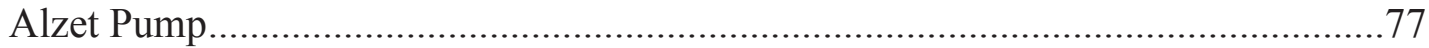

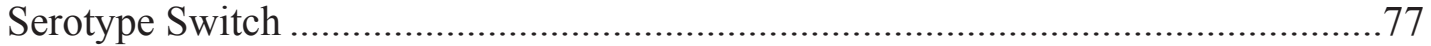

Test for Tumorigenicity of Pre-Infected Tumor Cells .............................................78

Pre-Infected Tumor Cell Carriers in Tumor Bearing Rats.......................................78

Recovery of Virus from PC-Treated Animals .......................................................78

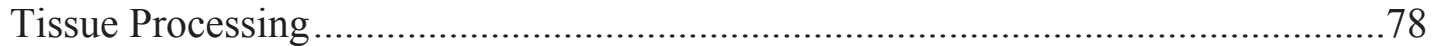

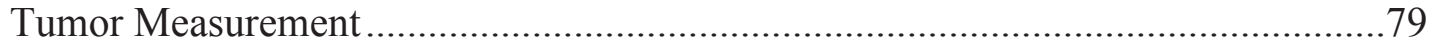

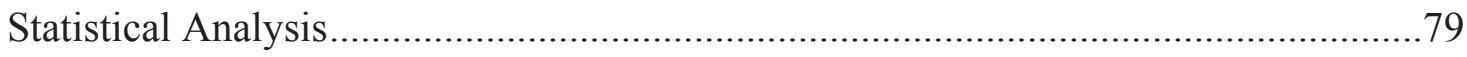

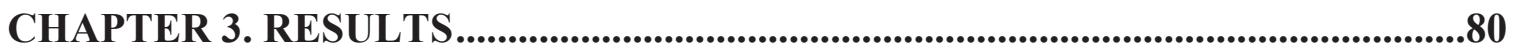

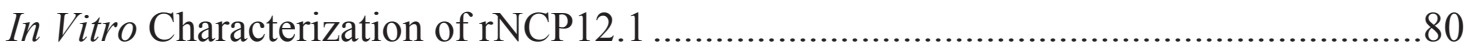

rNCP12.1 Retains Its Non-Cytopathic Phenotype in Normal Brain Cells While Remaining Cytopathic in Most Tumor Cell Lines. 
rNCP12.1 Upregulates IFN $\beta$ Expression and Increases Production of Active IFN over wtVSV in a Cell Specific Manner ....

IFN $\beta$ Gene Expression Does Not Correlate with Production of Active IFN in

Primary Rat Astrocytes

Primary Astrocytes Are Highly Sensitive to the Anti-Viral Effects of IFN While

Glioma Cells Are Not

IFN Activity Following rNCP12.1 Infection Is Associated with Induction of

ISGs in Primary Astrocytes While Glioma Cells Vary in Their Ability to Elicit

Downstream IFN Responses ...................................................................91

rNCP12.1 Alters the Temporal Expression of Ddx-58 (RIG-I) in Glial Cells ..........94

Efficacy of rNCP12.1 in an Immunocompetent Intracranial Model of Glioma ............96

Intracranial Administration of rNCP12.1 Is Safe Relative to wtVSV in Non-

Tumor Bearing Animals .................................................................................96

rNCP12.1 Decreases Tumor Load in a Dose Dependent Manner Without

Causing Morbidity in an Intracranial Model of C6-GFP Glioma ............................96

Comparison of the F98 Glioma/ Fischer Rat Model..............................................97

Intracranial Administration of rNCP12.1 Is Safe but Results in a Non-Durable

Reduction of Tumor Load in the F98 Glioma Model ...........................................100

One Intracranial Dose of rNCP12.1 Induces Both Innate and Adaptive Immune

Responses in F98 Glioma Bearing Rats by 15 Days Post-Infection.......................106

Evaluation of Various Methods of Viral Administration on Safety and Efficacy of

rNCP12.1 as an Oncolytic Treatment for Intracranial Glioma .................................110

Constant Infusion of rNCP12.1 over Time by Infusion Pumps Is Not a More

Effective Method of Viral Delivery

Switching Serotype Enhances Survival and Prolongs Tumor Reduction in F98

Glioma Bearing Animals

Administration of rNCP12.1 by Tumor "Carrier Cells" as a Potential Method of

Delivering Virus for the Treatment of Intracranial Glioma.

Infected tumor carrier cells are unable to form intracranial glioma

Pre-infected tumor cell carriers cause tumor load reduction with moderate morbidity at early time points with later time points inconclusive.

Infectious virus can be recovered from brain tissue of PC-treated animals ....... 126

Innate and adaptive antiviral immune responses were not increased following

carrier cell delivery

CHAPTER 4. DISCUSSION .............................................................................................138

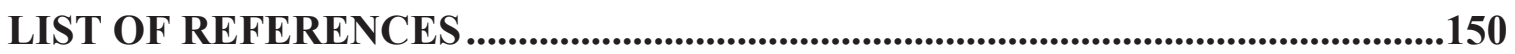

APPENDIX A. SUPPLEMENTARY FIGURES AND TABLES................................173

APPENDIX B. STANDARDIZING THE IFN BIOASSAY AFTER VSV

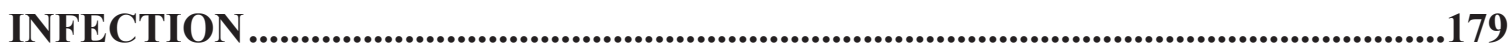

Introduction and Purpose .....................................................................................179

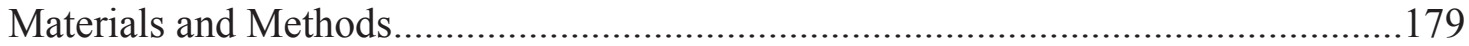

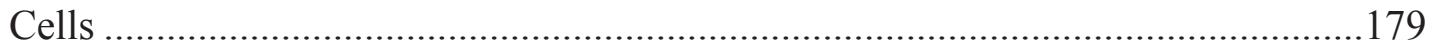




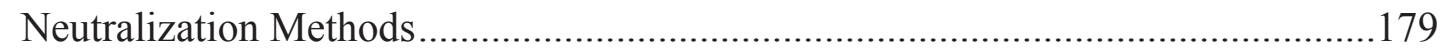

Neutralization by anti-VSV neutralizing antibody (TN-1) ................................ 180

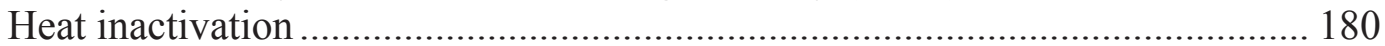

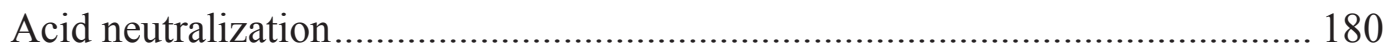

Combination of heat inactivation and acid neutralization ................................. 180

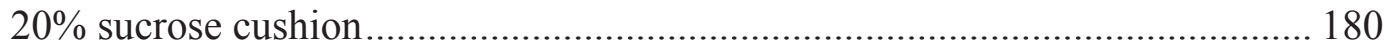

Combination of $20 \%$ sucrose cushion and acid neutralization ........................... 180

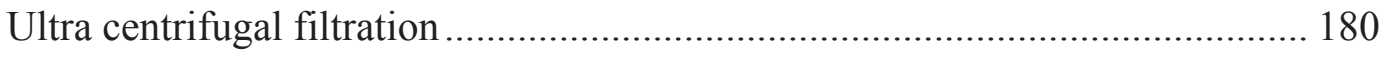

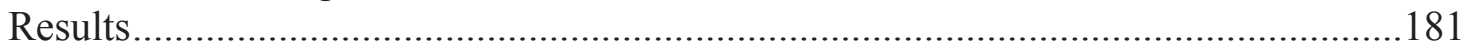

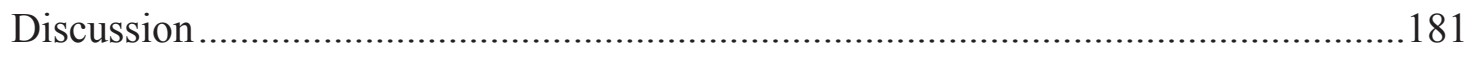

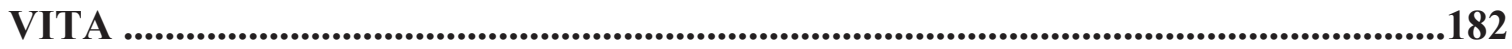




\section{LIST OF TABLES}

Table 1-1. Histological grading of astrocytic tumors as defined by the World Health Organization (WHO) ......................................................................

Table 1-2. Chromosomal aberrations significantly associated with molecular subtypes of glioblastoma multiforme

Table 1-3. Current oncolytic viral therapies in clinical trials for the treatment of GBM.

Table 1-4. Functional domains of VSV matrix protein ............................................44

Table 1-5. VSV-derived oncolytic viruses for the treatment of GBM ..........................58

Table 1-6. Characterization of individual mutations in matrix mutants........................64

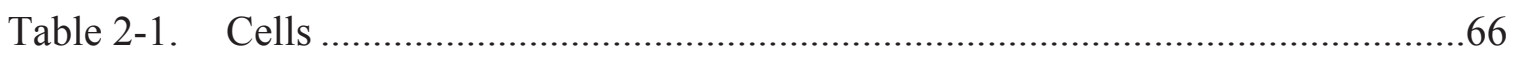

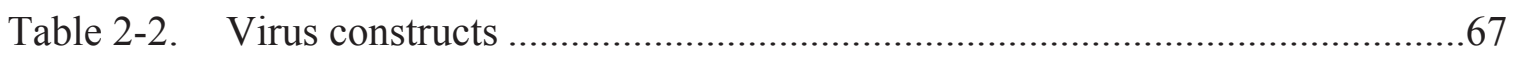

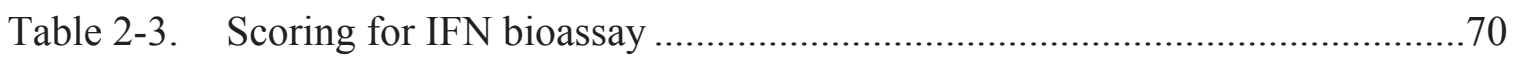

Table 2-4. qRT-PCR UPL primers for interferon stimulated genes............................ 71

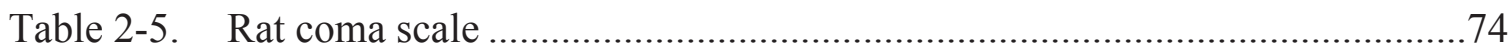

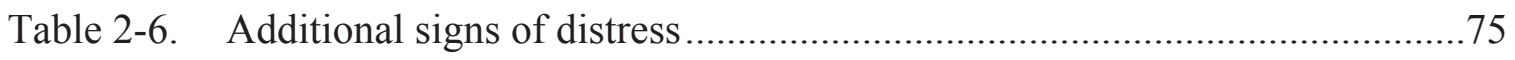

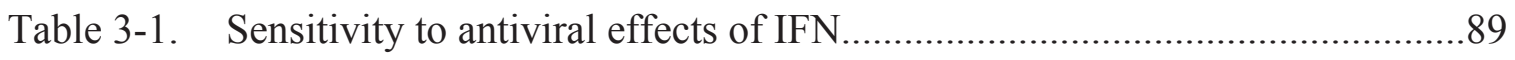

Table 3-2. Description of treatment groups in Alzet pump study ............................113

Table 3-3. Summary of survival for animals treated in Alzet pump study relative to

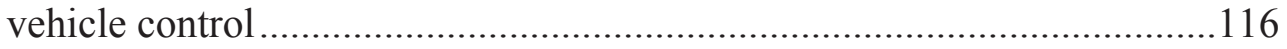

Table 3-4. Description of treatment groups in Indiana/New Jersey double injection

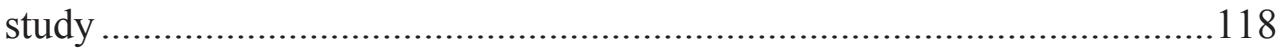

Table 3-5. Description of treatment groups in evaluation of pre-infected tumor cell implantation study.

Table 3-6. Description of treatment groups in rNCP12.1-GFP pre-infected cell study

Table 4-1. Summary of in vitro results of VSV in normal and tumor glial cells 
Table 4-2. Summary of in vivo experiments of rNCP12.1 in the syngeneic F98 glioma/ Fischer rat model......................................................................147

Table A-1. qRT-PCR UPL primers for housekeeping genes ..........................................174 


\section{LIST OF FIGURES}

Figure 1-1. Distribution of primary brain tumors.......................................................

Figure 1-2. Description of the multi-phase drug approval process ..............................

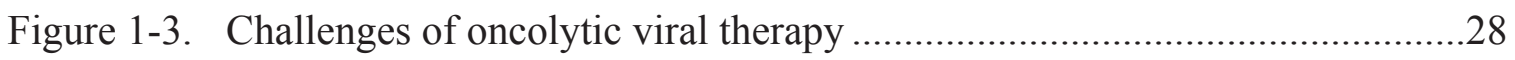

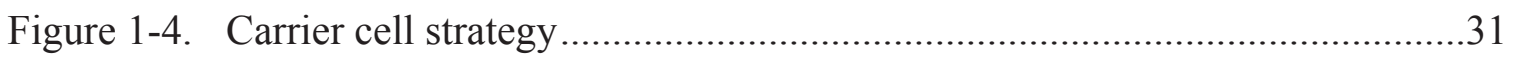

Figure 1-5. Select representatives of the order, Mononegavirales .................................33

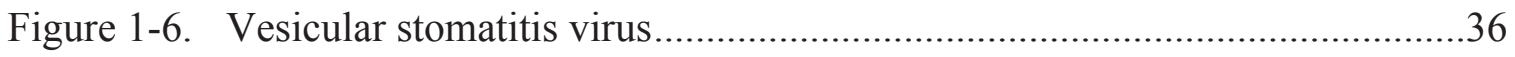

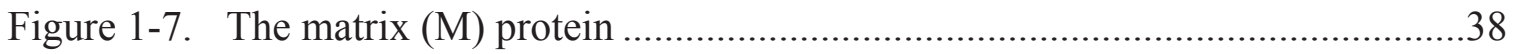

Figure 1-8. Multiple steps in the inhibition of host gene expression by VSV matrix

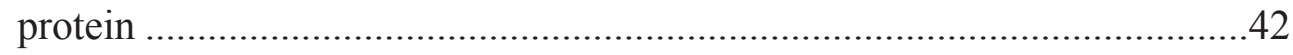

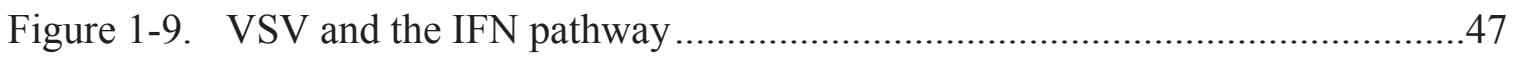

Figure 1-10. Cell rounding phenotype of rNCP12 in different cell types ......................63

Figure 3-1. Cell rounding phenotype of rNCP12.1 in human glioma cells ....................81

Figure 3-2. Characterization of rNCP12.1 in glioma cells ......................................82

Figure 3-3. Cell and viral specific comparisons of IFN responses.............................85

Figure 3-4. Representative fluorescence micrographs showing infection of cells with wtVSV following IFN pre-treatment ...............................................90

Figure 3-5. Differences in interferon stimulated genes (ISGs) following viral infection with wtVSV or rNCP12.1-GFP ...........................................92

Figure 3-6. Heat map representation of IFN stimulated gene (ISG) expression in normal and tumor glial cells following viral infection..............................95

Figure 3-7. Dose-dependent reduction of C6 tumors after rNCP12.1 treatment............98

Figure 3-8. Comparison of the C6 and F98 rat intracranial glioma models.................101

Figure 3-9. Tumor progression of F98-GFP glioma in Fischer rat over time ...............102

Figure 3-10. Timeline for the single rNCP12.1- GFP injection study in Fischer rat ......103

Figure 3-11. Comparisons of tumor properties in animals treated with a single i.t injection of rNCP12.1-GFP. 
Figure 3-12. Measures of morbidity of animals treated with a single i.t. injection of rNCP12.1-GFP

Figure 3-13. Detection of immune responses at day 15 in animals treated with a single injection of rNCP12.1 ......................................................... 108

Figure 3-14. Stability of rNCP12.1 during the use of Alzet infusion pumps .................111

Figure 3-15. Timeline for Alzet pump delivery study ............................................113

Figure 3-16. Comparison of tumor load in animals treated with varying doses of rNCP12.1 delivered by Alzet pump infusion ........................................114

Figure 3-17. Time line of Indiana/New Jersey double injection study.........................118

Figure 3-18. Evaluation of animals treated with Indiana/Indiana versus Indiana/New Jersey double injection

Figure 3-19. Morbidity of animals treated in the Indiana/Indiana versus Indiana/New Jersey double injection study

Figure 3-20. Timeline for evaluation of pre-infected tumor cell implantation study ......123

Figure 3-21. Evaluation of tumor implantation by rNCP12.1-GFP pre-infected F98 glioma.

Figure 3-22. Timeline of rNCP12.1-GFP pre-infected cell study

Figure 3-23. Evaluation of rNCP12.1-GFP pre-infected F98 cells as treatment in rat

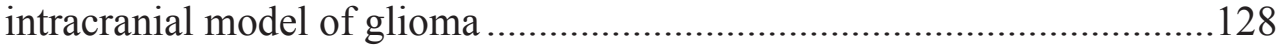

Figure 3-24. Detection of virus in PC- and FV- treated animals

Figure 3-25. Evaluation of innate and adaptive immune responses following treatment with pre-infected tumor cells 135

Figure 4-1. Interferon phenotypes of glial cells in response to rNCP12.1 infection.....149

Figure A-1. Expression of housekeeping genes in FR cells ...................................... 173

Figure A-2. Expression of Cyclophilin B in infected FR cells...................................175

Figure A-3. Test of UPL primer sets for the expression of ISGs in multiple cell lines .176

Figure A-4. BCA protein assay of proteins derived from glial cell lysate. 177

Figure A-5. Detection of circulating anti-tumor antibodies in animals treated with pre-infected cells versus free virus. 


\section{LIST OF ABBREVIATIONS}

\begin{tabular}{|c|c|}
\hline AV & attenuated virus \\
\hline BBB & blood brain barrier \\
\hline CBTRUS & Central Brain Tumor Registry of the United States \\
\hline $\mathrm{CD}$ & cytosine deaminase \\
\hline CEA & carcinoembryonic antigen \\
\hline CED & convection enhanced delivery \\
\hline CNS & central nervous system \\
\hline CPE & cytopathic effects \\
\hline CRAd & conditionally replicating adenovirus \\
\hline CSF & cerebrospinal fluid \\
\hline D5 (D10) & DMEM $+5(10) \%$ fetal bovine serum \\
\hline Ddx-58 & DEAD box polypeptide 58 \\
\hline FR & rat fibroblasts \\
\hline FV & free virus \\
\hline G & glycoprotein \\
\hline GBM & glioblastoma multiforme \\
\hline GBP1 & guanylate binding protein 1 \\
\hline GCV & ganciclovir \\
\hline GFAP & glial fibrillary acidic protein \\
\hline GFP & green fluorescent protein \\
\hline $\mathrm{G}_{\mathrm{I}}$ & Indiana glycoprotein \\
\hline $\mathrm{G}_{\mathrm{NJ}}$ & New Jersey glycoprotein \\
\hline hpi & hours post-infection \\
\hline HSV & herpes simplex virus \\
\hline i.t. & intratumoral \\
\hline i.v. & intravenous \\
\hline IFN & interferon \\
\hline IRF1 & interferon regulatory factor 1 \\
\hline ISG & interferon stimulated genes \\
\hline ISRE & IFN-stimulated response element \\
\hline JAK & janus kinase \\
\hline $\mathrm{L}$ & large RNA polymerase \\
\hline M & matrix \\
\hline $\mathrm{mAb}$ & monoclonal antibody \\
\hline MEF & mouse embryonic fibroblasts \\
\hline MGMT & O6-methylguanine-DNA methyltransferase \\
\hline MOI & multiplicity of infection \\
\hline MV & measles virus \\
\hline Mx & myxovirus resistance \\
\hline $\mathrm{N}$ & nucleoprotein; nucleocapsid protein \\
\hline $\mathrm{nAb}$ & neutralizing antibody \\
\hline NCT & nucleocytoplasmic transport \\
\hline NDV & Newcastle disease virus \\
\hline
\end{tabular}




$\begin{array}{ll}\text { NPC } & \text { nuclear pore complex } \\ \text { OV } & \text { oncolytic virus } \\ \text { P } & \text { phosphoprotein } \\ \text { PBS } & \text { phosphate buffered saline } \\ \text { PC } & \text { pre-infected cells } \\ \text { PFU } & \text { plaque forming units } \\ \text { pI:C } & \text { polyinosinic: polycytidylic acid } \\ \text { PKR } & \text { protein kinase R } \\ \text { Plscr1 } & \text { phospholipid scramblase 1 } \\ \text { PRA } & \text { primary rat astrocytes } \\ \text { PRR } & \text { pattern recognition receptor } \\ \text { PV } & \text { poliovirus } \\ \text { qRT-PCR } & \text { quantitative reverse transcriptase- polymerase chain reaction } \\ \text { Rabv } & \text { rabies virus } \\ \text { RCS } & \text { rat coma scale } \\ \text { RIG-I } & \text { retinoic acid-inducible gene-I } \\ \text { rNCP12.1 } & \text { recombinant non-cytopathic VSV, isolate 12.1 } \\ \text { RNP } & \text { ribonucleoprotein } \\ \text { RVV } & \text { retroviral replicating vector } \\ \text { SF } & \text { serum free } \\ \text { STAT } & \text { signal transducers and activators of transcription } \\ \text { TAA } & \text { tumor associated antigen } \\ \text { TK } & \text { thymidine kinase } \\ \text { TLR } & \text { Toll-like receptor } \\ \text { TMZ } & \text { temozolamide } \\ \text { TN } & \text { tris-saline buffer } \\ \text { VCV } & \text { valaciclovir } \\ \text { VSV } & \text { vesicular stomatitis virus } \\ \text { WHO } & \text { World Health Organization } \\ \text { wt } & \text { wildtype } \\ & \end{array}$




\title{
CHAPTER 1. INTRODUCTION
}

\author{
Glioblastoma Multiforme
}

\section{Definition and Classification}

Gliomas are both benign and malignant neuroepithelial tumors originating from the supporting glial cells of the central nervous system (CNS) [1]. The accepted standard for defining tumors of the CNS has been established by The World Health Organization (WHO) histological grading system, which subdivides tumors based on the glial cell from which they most likely derive including cells of astrocytic, oligodendroglial, mixed oligoastrocytic, or ependymal nature. Particularly, astrocytic tumors can be classified into four distinct histological groups corresponding to their level of malignancy (See Table 1-1). Grade I tumors such as pilocytic astrocytomas have low proliferative potential and are the least malignant. Grade II neoplasms are generally of low proliferative potential yet have a higher capacity to infiltrate surrounding normal tissue. For this reason, Grade II lesions often recur as high-grade tumors following initial treatment. Grade III and IV tumors are considered high grade, and, particularly, WHO grade III tumors are anaplastic lesions that are histologically malignant, displaying nuclear atypia and high mitotic activity. Lastly, WHO grade IV describes highly malignant tumors with similar histological features of grade III tumors but with enhanced proliferation of microvasculature and necrotic centers often surrounded by hypercellular areas called pseudopalisades which are thought to be cells migrating away from hypoxic areas of tumor [2]. Included in the Grade IV category are glioblastomas, formerly known as glioblastoma multiforme or GBM, the most detrimental of high-grade gliomas [3].

Table 1-1. Histological grading of astrocytic tumors as defined by the World Health Organization (WHO)

\begin{tabular}{ll}
\hline \multicolumn{1}{c}{ Histological Grade } & \multicolumn{1}{c}{ Tumor (s) } \\
\hline I & $\bullet$ Subependymal Giant Cell Astrocytoma \\
II & $\bullet$ Pilocytic Astrocytoma \\
& $\bullet$ Pilomyxoid Astrocytoma \\
& - Diffuse Astrocytoma \\
III & - Pleomorphic Xanthoastrocytoma \\
IV & - Anaplastic Astrocytoma \\
& $\bullet$ Giablastoma (Glioblastoma Multiforme) \\
& $\bullet$ Gliosarcoma \\
\hline
\end{tabular}




\section{Epidemiology}

According to the Central Brain Tumor Registry of the United States (CBTRUS), there were 311,202 primary brain and CNS tumors diagnosed during 2005-2009; 201,507 of which were malignant. Gliomas comprise $30 \%$ of all CNS tumors and $80 \%$ of all malignant tumors. Of gliomas, GBM makes up over 50\% and is the second most commonly diagnosed $(16 \%)$ primary tumor second only to the nonmalignant meningioma (35\%) (Figure 1-1). Of CNS malignancies, GBM ranks first. Males are typically affected more than females at a ratio of 1.58 , and whites are twice as likely to be diagnosed with GBM over African Americans. The median age of diagnosis for GBM is 64 with highest rates seen in those over the age of 75 making it a disease of advanced age. As the most malignant and notorious of brain tumors, mortality is high following diagnosis. One-year survival is estimated at $35.7 \%$ dropping down to $5 \%$ at 5 years $[4,5]$.

Cause(es) of glioma are largely unknown. Because gliomas are a disease seen more prevalently in industrialized countries, many studies have attempted to find a relationship between environmental factors such as cell phone usage. Unfortunately, none have been convincing other than demonstrating an increased risk with past history of radiotherapy to the head and/or neck. There are known hereditary syndromes associated with increased risk of glioma, but these are rare. Examples of these include LiFraumeni syndrome, familial adenomatous polyposis syndrome (FAPS), Lynch syndrome, and Neurofibromatosis (NF) 1, each of which have been associated with single gene mutations [6].

\section{Diagnosis}

Signs and symptoms are important in the diagnosis of CNS tumors. Those that raise suspicion include constitutional symptoms such as fatigue and headache as well as dizziness, confusion, memory loss, and behavioral changes. Focal neurological symptoms may be present and include changes in vision, speech, and motor or sensation, which vary based on tumor location. Additionally, symptoms indicative of increased intracranial pressure from mass effect of tumor may be present and include headache associated with vomiting most noticeable upon awakening. Radiological studies are extremely important to the diagnosis of GBM and aid in confirming tumor malignancy. These include computed tomography (CT) or magnetic resonance imaging (MRI), the latter being the study of choice as it is more sensitive to specific features of malignancy such as peritumoral edema and areas of abnormal intensity that correspond to foci of tumor cell infiltration. Often areas of central necrosis are visualized making high grade tumors a more likely diagnosis than low grade [7].

\section{Standard Treatments for GBM}

Regardless of etiology, prognosis for GBM patients is grim with survival ranging from 3-15 months, depending on available treatment options. Negative prognostic factors 


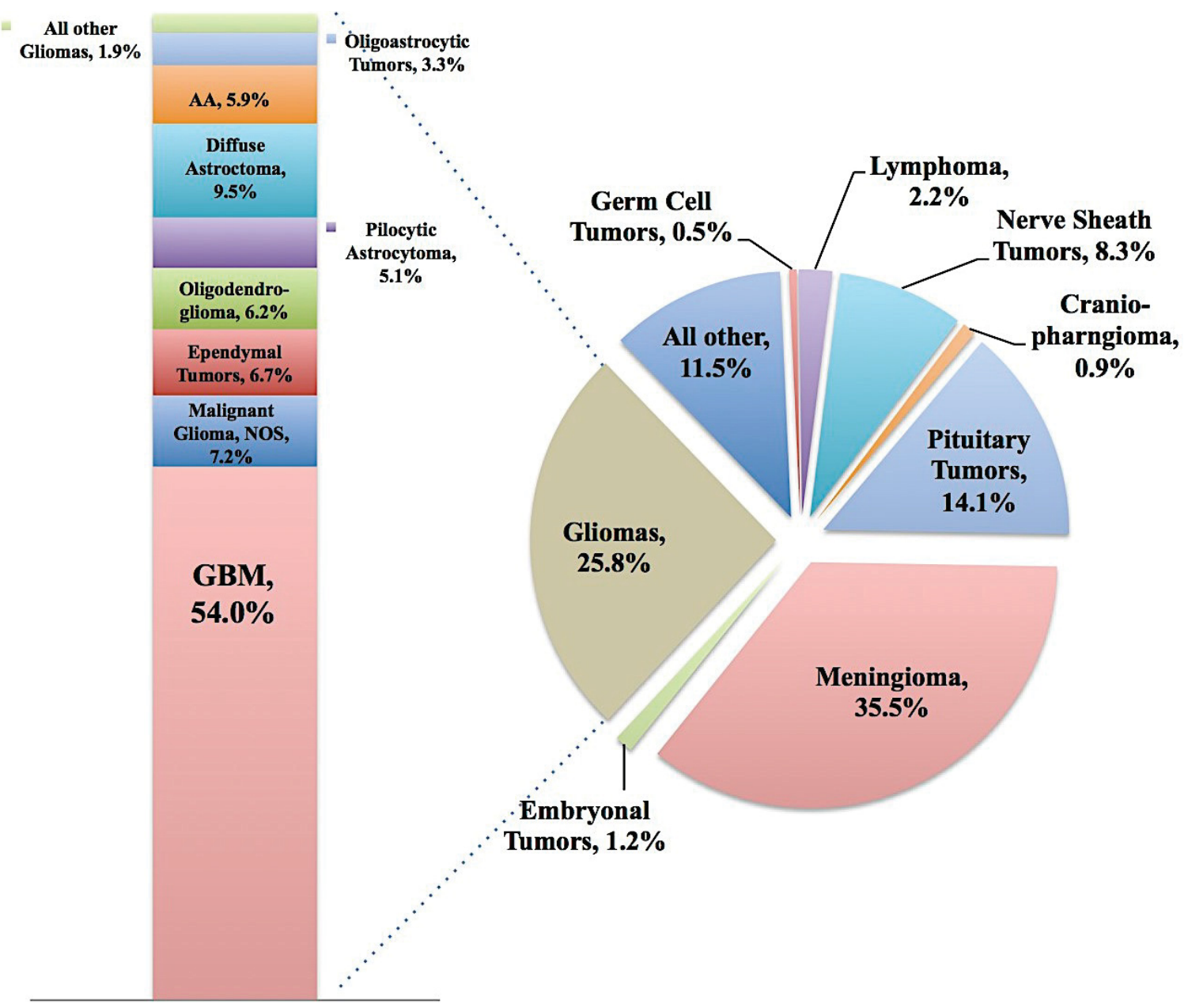

\section{Figure 1-1. Distribution of primary brain tumors}

The distribution of primary brain tumors based on histology are depicted in the pie graph. The bar graph illustrates distribution of glial specific tumors.

Source: Dolecek T, J. Propp, N. Stroup, and C. Kruchko (2012). CBTRUS Statistical Report: Primary Brain and Central Nervous System Tumors Diagnosed in the United States in 2005-2009. Neuro-oncology, 14:1-49 [4]. 
include advanced age, infiltrative histological features, poor performance status (Karnofsky scale), and inability to surgically debulk tumor. For low-grade tumors, prognosis is best with 5yr survival of Grade I tumors averaging $94 \%$ as tumors are usually cured by surgery alone. On the other hand, surgery is often not feasible for Grade IV tumors due to infiltration especially into eloquent areas of the brain. Other factors affecting ability to operate include advanced age and comorbidities. Regardless, the standard treatment for newly diagnosed GBM includes surgical resection, radiotherapy, and adjuvant chemotherapy.

Surgical management not only provides tissue for diagnosis as well as relief from symptoms caused by mass effect, but the extent of resection (EOR) has been shown to directly influence overall survival. In a retrospective review of GBM patients who underwent 2 or more craniotomies for tumor resection, outcomes based on gross total resection (GTR) versus subtotal resection (STR) at time of diagnosis and recurrence were compared and a significant improvement in survival was associated with debulking of at least $78 \%$ of tumor mass. These numbers improved with increased percentage of resection [8]. Current studies are focusing on improving both surgical techniques and imaging strategies in order to enhance this positive effect on survival for patients who qualify for resection [9]. Even further, the combination of surgery and radiation was found to increase survival [10]. Conventional radiotherapy regimens consist of $60 \mathrm{~Gy}$ of partial-field external- beam irradiation delivered 5 days per week in fractions of 1.8 to 2.0 Gy [7]. Since the implementation of chemotherapy as part of the standard of care, survival has increased from 12 to 15 months. Until 2005, there were a number of drugs evaluated and often experimentally used in the clinical setting as chemotherapy agents for GBM. These included agents such as procarbazine, a DNA alkylating agent, and the nitrosourea chemotherapies such as carmustine (BCNU) or lomustine (CCNU). In 2005 following a groundbreaking study by the European Organisation for Research and Treatment of Cancer (EORTC) and the National Cancer Institute of Canada (NCIC) on treatment of newly diagnosed GBM, another chemotherapy agent, Temozolomide (TMZ) became the agent of choice as this study showed an increase in survival of 3 months when TMZ was added to radiation versus radiation alone. TMZ is an oral prodrug agent that is metabolized into its active form, which then methylates DNA at a number of sites causing DNA damage and subsequent cellular apoptosis. As with many chemotherapies, GBMs become resistant to TMZ therapy by several mechanisms that involve dysregulation of DNA repair systems. Unfortunately, the ability to treat with TMZ is largely limited by its myelosuppressive effects [11].

Despite the most aggressive conventional therapeutic regimen, a majority of tumors eventually recur within 7 months $[7,12]$. These tumors are typically more aggressive due to newly acquired genetic changes in the tumor cell population promoting resistance to radio- and chemotherapies [13]. 


\section{Molecular Subtypes of Glioblastoma}

In accordance with the WHO system, histological characteristics are evaluated to classify gliomas based on glial origin and malignant potential. This grading system often falls short in its ability to predict prognosis and response to treatments, as many histologically similar gliomas respond very different to treatment. This may be due to technical error as histological grade is dependent upon interpretation by neuropathologists and borderline histological features often interfere with this interpretation [1]. On the other hand, underlying genetic profiles of histologically similar tumors may cause them to behave very differently in response to treatment and therefore affect long-term survival. The observation that certain Grade IV gliomas have increased susceptibility to TMZ based on the presence or absence of mutations in a DNA repair enzyme promoter is an example of this genetic influence. O6-methylguanine-DNA methyltransferase (MGMT) is a DNA repair enzyme that works by inhibiting cross linking of dsDNA which prevents the action of alkylating chemotherapeutic agents and is often responsible for resistance of tumors to these agents. When methylated, the MGMT promoter is silenced therefore increasing the tumor's susceptibility to TMZ. Studies have shown that survival with TMZ therapy is enhanced to 21.7 months versus 12 months in patients with unmethylated MGMT [7].

It is understood that GBMs are composed of a heterogeneic population of cells, and therefore several genetic subtypes are currently recognized [13]. Primary GBM makes up $90 \%$ of total GBMs diagnosed and arises de novo without a history of a lower grade precursor lesion. Primary tumors exhibit a number of frequent genetic alterations including EGFR amplification, homozygous deletion of the CDKN2A gene coding for both p16INK4a and/or p14ARF, and chromosome 10 or PTEN mutations. In contrast, secondary GBMs, comprising only $10 \%$ of total GBMs, arise from a lower-grade precursor lesion and usually carry TP53 and IDH1 mutations in more than two thirds of cases. Other common genetic alterations of secondary GBMs include allelic losses on $19 \mathrm{q}$ and $13 \mathrm{q}$, promoter hypermethylation of the $R B 1$ gene, and overexpression of $P D G F R-A$ (reviewed in [6]). Taken together, these data clearly indicate that primary and secondary GBMs represent genetically different diseases yet both share similar histological feature [14] and while this characterization has not been shown to have an association with prognosis, it has been exploited in developing more targeted therapies [13].

This discovery of genetically associated subtypes for GBM has prompted even more sophisticated analyses that have uncovered more altered genes and pathways likely contributing to tumor behavior. Of note is The Cancer Genome Atlas (TCGA) Research Network established in 2006 to generate a comprehensive database of genomic abnormalities in various cancers. Studies derived from information in this database, have exposed other associations leading to the proposal of additional genetic classes of GBM. One particular study revealed four well-defined genetic subtypes of GBM: Proneural, Neural, Classical, and Mesenchymal. These subtypes are characterized based on the following genetic profiles: Classical is associated with EGFR overexpression, PTEN loss, and to a lesser extent chromosome 9p21 deletions targeting p16INK4a and p14ARF; 
Mesenchymal with NF1; Proneural with PDGFRA, IDH-1, and TP53 mutations; Neural with the presence of neuronal markers such as NEFL, GABRA1, SYT1, and SLC12A5

(Table 1-2). Other defining features of these subtypes include differences in responses to standard therapies with the greatest response seen in Classical GBM and no benefit seen with Proneural subtype. On the other hand, the classical subtype is usually associated with increased age, a known negative prognostic factor while Proneural tumors are associated with younger age [15].

This and subsequent studies prove that gene expression-based grouping of tumors can be a more powerful prognostic and predictive factor than histologic grade or age by revealing unrecognized heterogeneity of histologically similar tumors. Even newer class subtypes are being uncovered that not only seek to determine differences in expression of individual gene mutations, but also, groups of genes to identify pathways that may be dysregulated in a specific tumor type. One such study has shown the enrichment of gene subsets that merge onto targets such as NFkB1 and EGFR [16-18].

\section{Importance of Chromosome 9 and IFN}

One pathway that has been studied for its role in tumorigenesis is the IFN pathway. In the early 1990s the hunt for tumor suppressor genes increased as many searched to uncover genes associated with deleted chromosomal material in a number of tumors. One particular deletion occurred on chromosome 9 and was found to be associated with multiple primary tumors and cell lines such as malignant human $\mathrm{T}$ cell lines, ALL, and melanoma [19-21]. Early on, the genetic material lost or affected was thought to be the interferon (IFN) gene cluster as it is located on the commonly deleted region of the short arm of chromosome 9,9p21. One of the first studies looking at the relationship between cancer and chromosome 9 deletions included a study by Collins et al 1991 that revealed deletions of 9p were found most likely in high grade tumors and further mapping revealed the locus to involve IFN $\alpha / \beta$ [22]. This was confirmed by subsequent studies that showed a prevalence of IFN gene deletions and/or sequence rearrangements in human glioma cell lines and high-grade primary and recurrent tumors $[23,24]$.

Others proposed that the IFN gene clusters may be affected due to their proximity to another gene whose function could be important in tumorigenesis. After the discovery of $\mathrm{p} 16 / \mathrm{ink} 4 \mathrm{a}$ an inhibitor of $\mathrm{pRb}$ phosphorylation through negative regulation of cyclin $\mathrm{D} / \mathrm{cdk} 4$ [25] and its location on chromosome 9 [26] the focus shifted from IFN to p16/ink4a as a culprit of malignancy in a number of GBM tumors [27-30]. In fact, more recent studies based on TCGA database confirm that the classical subtype of GBM which harbors mostly EGFR mutations also expresses deletions of 9p21 targeting p16/INK4a and p14ARF [15]. These two mutations occur simultaneously in 94\% of GBMs tested.

Despite p16's correlation with chromosome 9 deletions, we still know that IFN is affected and that defects in the IFN pathway provide a survival advantage over normal cells. Many therapies have been developed exploiting either the growth inhibitory 
Table 1-2. Chromosomal aberrations significantly associated with molecular subtypes of glioblastoma multiforme

\begin{tabular}{|c|c|c|c|c|c|c|}
\hline \multirow{2}{*}{\multicolumn{2}{|c|}{$\begin{array}{c}\text { Chromosomal } \\
\text { Aberration* }\end{array}$}} & \multirow{3}{*}{$\begin{array}{c}\begin{array}{c}\text { Gene } \\
\text { Affected }\end{array} \\
\text { EGFR }\end{array}$} & \multicolumn{4}{|c|}{ GBM Subtype } \\
\hline & & & Classical & Mesenchymal & Neural $^{\S}$ & Proneural \\
\hline $7 \mathrm{p} 11.2$ & $\uparrow$ & & & & & $\checkmark$ \\
\hline $7 \mathrm{q} 21.2$ & $\uparrow$ & CDK6 & & & & $\checkmark$ \\
\hline $7 \mathrm{q} 31.2$ & $\uparrow$ & MET & & & & $\checkmark$ \\
\hline $7 \mathrm{q} 34$ & $\uparrow$ & - & & & & $\checkmark$ \\
\hline $7 \mathrm{p} 11.2$ & 个个 & EGFR & $\checkmark$ & & & $\checkmark$ \\
\hline $4 q 12$ & 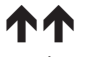 & PDGFRA & & & & $\checkmark$ \\
\hline $17 \mathrm{q} 11.2$ & 1 & NF1 & & $\checkmark$ & & \\
\hline $10 \mathrm{q} 23$ & 1 & PTEN & & & & $\checkmark$ \\
\hline $9 \mathrm{p} 21.3$ & I & CDKN2A/ & $\checkmark$ & & & \\
\hline & & CDKN2B & & & & \\
\hline $13 q 14$ & 1 & RB1 & $\alpha$ & & & \\
\hline $9 \mathrm{p} 21.3$ & $\times$ & CDKN2A/ & $\checkmark$ & & & \\
\hline & & CDKN2B & & & & \\
\hline
\end{tabular}

*ヘ $=$ Low level amplification; $\boldsymbol{\uparrow}=$ High level amplification; /= Hemizygous deletion;

$X=$ Homozygous deletion

${ }^{\S}$ Neural subtype is significantly associated with presence of neuronal markers, not specific chromosomal aberrations.

Source: Verhaak, R, K. Hoadley, E. Purdom, V. Wang (2010). Integrated genomic analysis identifies clinically relevant subtypes of glioblastoma characterized by abnormalities in PDGFRA, IDH1, EGFR, and NF1. Cancer Cell. 17: 98-110 [15]. 
actions of IFN on tumor cells or the inability of tumor cells to produce protective IFN responses. Heymann et al demonstrated a correlation between changes in genetic material and IFN bioactivity in malignant $\mathrm{T}$ cell lines showing that several cell lines with normal dosage of IFN genes had deficiencies in producing IFN or in IFN sensitivity [20].

\section{Experimental Therapies for GBM}

Though cure is the ultimate goal, with a median survival of only 12-15 months post-diagnosis, therapies for GBM that are able to prolong progression free survival (PFS) with a high quality of life are warranted. There are a number of studies occurring at various stages of the drug approval process that are aimed at doing just this (Figure 1-2). These not only involve experimental therapies that exploit newly defined genetic characteristics of GBM, but they also involve the search for more efficient methods of administering existing chemotherapeutic agents. Because GBM is a "local" disease in that there have been very few confirmed instances of extra-axial metastases, possibly due to short survival times post-diagnosis, improved methods of local administration are being developed [31-33]. Gliadel, a carmustine (BCNU)-containing implantable degradable polymer wafer, is FDA approved as an adjuvant treatment with surgery and radiation of newly diagnosed GBM as well as an adjuvant to surgery in patients with recurrent disease. Its safety was proven in phase I trials and, later, phase III trials demonstrated an increase in survival by 8 weeks versus placebo wafers. Advantages of the system include the ability to bypass administering toxic levels of drug needed to cross the blood brain barrier when carmustine is given systemically therefore preventing side effects such as myelosupression. On the other hand, wafers have been associated with increased intracranial pressure caused by malignant edema, seizures, and post implantation infections [34]. Another example of a local delivery system for GBM includes Convection-Enhanced [intratumoral] Delivery (CED). This system involves stereotactically implanted catheters that deliver therapy by high flow infusion over a specified period of time. Advantages of this system are its ability to pump drug uniformly through the extracellular matrix over large areas of brain. Catheters can be placed either within tumors or in the tumor bed after surgical resection. Unfortunately, CED has been limited to small supratentorial tumors away from the sagittal sinus located in the midline [35]. Also, in theory, drug distribution should be uniform however distribution has often been found to be variable and unpredictable with CED delivery [36]. Nevertheless, a number of chemotherapy agents as well as targeted drugs such as cytotoxins have been tested using this mode of delivery [37].

Though chemotherapy is an accepted part of the therapeutic regimen for GBM, the agents are broadly acting and function by damaging DNA in both tumor and normal cells. On the other hand, targeted therapies work by selectively killing tumor cells according to differences in expression of genetic pathways and markers. For example, we know that EGFR overexpression is found in $~ 50 \%$ of GBMs and the receptors are constitutively active in $1 / 2$ of these. This path has been shown to be the major genetic aberration in the classical genetic subtype of GBM and is the major genetic feature of primary GBM tumors. Therapies that target EGFR have been studied in several Phase 


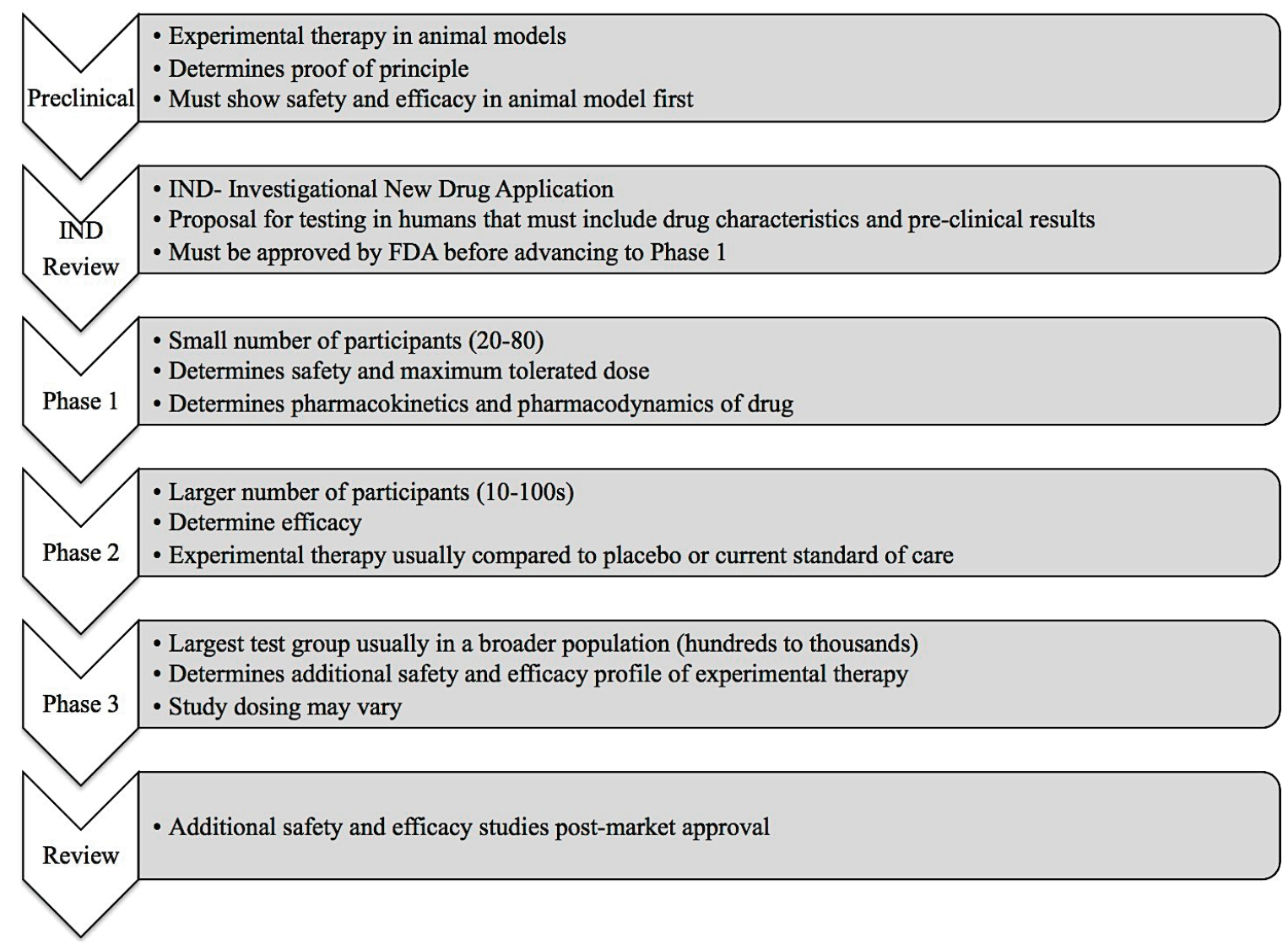

Figure 1-2. Description of the multi-phase drug approval process

Source: Food and Drug Administration

(http://www.fda.gov/drugs/resourcesforyou/consumers/ucm143534.htm) 
I/II trials and include the tyrosine kinase inhibitor, gefitinib, as monotherapy and as combination with other chemotherapeutic agents [38-40]. These drugs are considered antitumor therapies as they specifically inhibit the growth of tumor cells through inhibiting their mitogen-signaling cascade. Unfortunately, results of clinical trials to date have not shown an increase in PFS or overall survival (OS) in patients treated with EGFR inhibitors for glioma but they have shown promising results for non-small cell lung cancer (NSCLC) in Phase III trials and these inhibitors are currently being marketed as first line of therapy in patients with specific EGFR activating mutations [41, 42]. There are a number of ways in which this pathway can become overactive in cancer other than at the receptor binding step therefore, other inhibitors directed against more downstream signaling factors such as PI3k/akt/mTOR have been developed and are subjects of current clinical trials including combination therapy with direct inhibitors of EGFR [43, 44].

Immunotherapy, another novel therapeutic strategy for treating GBM, functions by using the body's immune cells and signals such as cytokines as cancer fighting agents. There are two types of immunotherapy, passive immunotherapy and active immunotherapy. Passive immunotherapy is the application of immunological agents such as antibodies or cytokines that act against tumor cells without directly activating the immune system. For instance monoclonal antibodies (mAb) directed against TAAs can be used as immunotherapy and one such agent has been FDA approved for the treatment of glioblastoma. Bevacizumab is a human $\mathrm{mAb}$ directed against the VEGF ligand, a major player in the dysregulation of angiogenesis of glioblastomas. This antibody sequesters VEGF and prevents binding to its receptor on the endothelium. Another example of passive immunity is the adoptive transfer of immune cells such as lymphocyte activated killer (LAK) cells or cytotoxic T lymphocytes (CTLs) that are activated, ex vivo, and then reintroduced to the patient either systemically, intratumorally, or into the tumor bed after surgical resection. As is the case of LAKs, these cells undergo cytokine-dependent activation, which is usually non-specific since cells are never exposed directly to tumor-associated antigens (TAAs). Alternatively, cells can be activated by exposure to TAAs as occurs with adoptive immunity involving CTLs which are then re-administered to the patient causing direct cytotoxicity upon contact with tumor (reviewed in [45]).

Alternatively, active immunotherapy works by stimulating the immune system against tumor by injecting sources of TAAs such as short peptides, tumor lysates, or whole tumor cells, or by injecting immune cells such as dendritic cells (DCs) that have been coupled to and/or activated by TAAs. In a phase I study, DCs pulsed or co-cultured with autologous tumor lysate were used as an adjuvant vaccine therapy in patients with newly diagnosed and recurrent glioblastoma after surgery and radiation but during TMZ treatment. Results showed that median overall survival of vaccinated patients was 31.4 months from time of diagnosis and three year survival rate, $47 \%$ compared to the US average of 14 months and $7.8 \%$, respectively. These results were even more pronounced in patients with tumors of the mesenchymal subtype, a group of tumors shown to be less responsive to conventional therapies [46]. An ongoing randomized, multicenter phase III to be completed in June of 2013 will determine whether this immunotherapy (DCVaxBrain) is effective for newly diagnosed glioblastoma [47]. 
Viruses have also been utilized as targeted therapies for GBM. They can work by introducing genes specifically in tumor cells usually by non-replicating viral vectors; however, more recent studies have used replicating viruses as vehicles to augment gene therapy. In non-replicating vectors, genes that are crucial to viral replication are substituted by a therapeutic gene of interest [48]. Whether by replicating or nonreplicating vectors, possible genes that can be incorporated into the viral genome include suicide genes, immunomodulators, or tumor suppressors.

A suicide gene is one that codes for an enzyme necessary in the metabolism of a non-toxic pro-drug to its active, cytotoxic form [49]. One of the most studied examples is the combination of the Herpes Simplex Virus (HSV) enzyme thymidine kinase (TK) and the pro-drug form of nucleoside analog antiviral agents such as ganciclovir (GCV) or valaciclovir (VCV). These pro-drugs are phosphorylated by TK to their toxic form allowing them to target and kill actively proliferating cells. An example of this therapeutic regimen includes the conditionally replicative adenovirus AdV-TK with the antiviral VCV currently undergoing phase II trials in combination with radiation for the treatment of resectable glioblastoma [47, 48]. Another example of a suicide gene/prodrug combination includes the delivery of a yeast enzyme cytosine deaminase (CD) by viral vector. This enzyme converts 5-fluorocytosine (5-FC) pro-drug into its active metabolite, 5-fluorouracil (5-FU) [49]. A therapy using this suicide gene/prodrug combination is currently being studied in Phase I/II trials for recurrent glioblastoma using the Toca 511 retroviral replicating vector (RVV) expressing CD [48, 50].

Delivery of immune stimulatory genes has proven to be of importance considering the notion that the CNS is immune "privileged". This privilege is based on the absence of lymph vessels and nodes within the CNS, few antigen presenting cells and circulating $\mathrm{T}$ lymphocytes, as well as presence of the blood brain barrier (BBB), the gatekeeper of all that moves into and out of the CNS. Specialized immune cells however do exist within the CNS and include microglia that when active, serve as the brain's major APC $[45,51]$. Other antigen presenting cells such as dendritic cells are found hovering around areas associated with breaches in the BBB as in the sub-ventricular zone (SVZ) until they are activated. Naïve T cells are not found in the CNS [52-54] but are able to enter when activated peripherally. In GBM patients, the immune system is often suppressed not only because of therapeutic corticosteroids that aid in minimizing the risk of mass-effect caused by malignancy-associated edema but also because of higher levels of immune suppressive cells such as regulatory T cells (T-regs), known to be involved in self tolerance, and higher levels of immunosuppressive cytokines such as prostaglandin E2 (PGE-2), TGF- $\beta$, and IL-10 (reviewed in [55]) [56]. An example of a novel therapy that promotes anti-tumor responses includes the viral vector, M032, an HSV construct carrying the IL-12 gene. This cytokine has been shown to trigger T-helper 1 (Th1) responses that aid in the recruitment of antitumor immune cells such as CTLs and NKs $[57,58]$. Results in preclinical studies demonstrated inhibition of human xenograft brain tumors and treated animals experience prolonged survival, even when virus was administered in the hemisphere opposite the implanted tumor. Memory of antitumor immunity was confirmed by the inability of re-challenge with tumor cells to establish tumors [58]. A phase I trial is currently underway for the treatment of patients with 
recurrent GBM [59]. Lastly, tumor suppressors, genes that are often muted in tumor cells on the road to becoming malignant, can be delivered by viral vectors. For instance, the tumor suppressor p53 is found to be down-regulated in over half of secondary GBMs [60]. This has been shown to have a negative effect on susceptibility to radiation in these tumors. Ad-p53 vector is a replication-defective adenovirus vector with a deleted E1 replication gene that is substituted by the p53 tumor suppressor gene. This vector was shown to increase radiosensitivity of GBM cells in vitro [61]. In a dose-escalation, multicenter Phase I trial of intratumorally injected Ad-p53, functional p53 protein was found to be expressed in tumor tissue however transduction of tumor cells was limited, reaching only to within $5 \mathrm{~mm}$ of the injection site. This revealed an obstacle encountered by many of the non-replicating vectors, the issue of widespread distribution [62].

The push for more effective agents with a capacity to propagate through surrounding tissue turned the attention toward replication competent viruses, many of which have a natural affinity for replication in tumor cells. As I will discuss, these tumor selective viruses are broken down into two major categories, those that are naturally occurring and those that have been genetically engineered to grow preferentially in tumor cells [63].

\section{Oncolytic Viruses as Novel Treatments for Cancer}

\section{Definition and Classification}

Oncolytic viruses (OVs) are viruses that selectively replicate in and kill tumor cells. As a result of this selectivity, normal cells are typically unharmed as infection to these cells should be minimal. This selectivity is based on various characteristics of both tumor and virus. Viruses, either by genetically engineered alterations or by natural ability, are able to exploit cellular defects that promote tumorigenesis in cancer cells. While these defects offer growth enhancing and survival advantages, they, inadvertently, weaken defense mechanisms permitting the replication and take over of viruses [64]. As the virus hijacks the cell's transcriptional and translational machinery, tumor cells are left unable to maintain its own growth and eventually die by either apopotosis or tissue necrosis [65-68].

\section{History}

The idea that viruses could be used as therapy for cancer was observed and documented at the turn of the 20th century when a number of patients were found to have spontaneously regressing tumors after experiencing acute viral infections [69]. The most encountered cancer displaying this phenomenon were those of blood lineage however cases of regression were observed in solid tumors such as cervical carcinoma as well [69]. Possibly the first recorded account of a case involving viral-induced tumor regression was discussed during an oral presentation by Dr. George Dock, a Hematology 
professor from the University of Michigan who described a patient with acute leukemia that was discovered to have experienced a complete, though temporary, remission after a flu-like illness [70, 71]. Another popular case came from observations made by Dr. de Pace who presented at the International Cancer Congress in Paris in 1910 his findings involving a patient with cervical carcinoma who received an attenuated rabies vaccine after a dog bite and subsequently experienced regression of her tumor $[63,72]$. Building on these observations, a research duo at Sloan-Kettering in New York showed the potential of oncolytic viruses in vivo first with animal models then in human patients with the first clinical trial using Egypt 101, a virus thought to be West Nile virus isolated from sera of infected patients. The trial included patients with cancers ranging from melanoma to pancreatic adenocarcinoma and results showed that of 34 patients, 4 displayed tumor regression and 27 demonstrated systemic infection, 14 of which viral replication was observed in biopsied tumor samples [73]. Keeping with the momentum of these findings, another trial several years later involved 30 patients with epidermoid cervical carcinomas who received an intratumoral injection of another potential OV, adenovirus (known at that time as adenoidal-pharyngeal-conjunctival virus, APC). Results were surprising as $65 \%$ of treated tumors had areas of necrosis, with tumor shedding so extensive that some patients experienced severe hemorrhage (reviewed in [69]) [74]. Unfortunately, these results were short lived and over half of those treated died from cancer within a few months of treatment $[75,76]$.

Other studies were carried out using viruses such as mumps virus (1974) and hepatitis virus (1949) [77, 78]. Unfortunately, many of these studies brought negative attention to the field as safety concerns became paramount. Several viruses used, like hepatitis B virus and West Nile virus, are extremely virulent in humans in their nonattenuated forms. The means by which these viruses were obtained would have been considered unethical under our current standards. Often, samples for therapy were derived from infectious body fluids or tissues from patients with active viral infections (reviewed in [69]). As a result, not only was there a need for more defined standards for these trials but there was also a need for viral agents that were less toxic in humans. The 1990s brought with it a new age of discovery in molecular techniques such as recombinant DNA technology while interest in more novel cancer therapies in the research community grew. A renewed focus developed in the field of oncolytic virology leading to the use of engineered viral vectors for treatment of cancer. Replicationdefective viruses, including engineered adenovirus and retrovirus vectors, were evaluated. Unfortunately, these agents carried with them a number of problems including inadequate delivery and distribution within the tumor mass, and consequently, a lack of long-term efficacy. A push for effective agents with less toxic profiles has led to studies using more attenuated forms of replication competent viruses, such as Herpes Simplex Virus (HSV), Conditionally Replicating Adenoviruses (CRAds), and paramyxoviruses like Newcastle Disease virus and measles viruses (reviewed in [69]) [70]. 


\section{Examples and Mechanisms of Action}

Advantages of OV therapy include the ability to genetically modify or create "designer viruses" that have enhanced specificity for binding to and replicating in tumor cells. A unique property of replicating viruses is their individual growth kinetics. Their ability to replicate and produce viral progeny without re-administering additional virus is a kinetic advantage not possessed by conventional chemotherapy or by non-replicating viral vectors [70].

There are a number of viruses that have been and are currently being tested in both preclinical and clinical studies ranging from DNA to RNA viruses, animal to human viruses, and naturally occurring to genetically engineered viruses. Each have there own advantages and disadvantages. For instance, though sequelae of infection by human viruses such as HSV are well-recognized, the effects on therapy due to pre-existing immunity can potentially diminish efficacy of treatment. On the other hand, many animal viruses have come to the forefront as potential therapies and while it is less likely that humans have come into contact with these viruses, the concern, though unlikely, of species adaptation causing pandemics as has been seen with swine influenza remains a consideration [79]. Concerning glioma, viruses from all categories are currently being considered as potential therapies. As can be expected, the viruses with a longer history of use in cancer therapy such as HSV and AdV are further along in the clinical trial pipeline than others, however viruses such as VSV are beginning to emerge as plausible contenders. A search on clinicaltrials.gov in January 2013 revealed at least 10 ongoing or completed phase I and II clinical trials of oncolytic virotherapy for GBM using a number of viruses from HSV to retrovirus (Table 1-3).

\section{Adenovirus}

Adenovirus (AdV) is a non-enveloped linear dsDNA virus belonging to the Mastadenovirus genus of the family, Adenoviridae. AdV is commonly encountered in the human population especially in children under four years of age, and usually causes self-limited infections involving the upper respiratory tract, conjunctiva, and, less often, the gastrointestinal tract [80]. There are over 50 serotypes of AdV with serotype 5 being the most used as viral based therapies for cancer [81]. AdV is one of the more studied and understood oncolytic viruses and its ease of recovery and ability to alter virally coded proteins for tumor targeting serve as advantages of their therapeutic use [82, 83]. CRAds are engineered adenoviruses that harbor partial or complete deletion of viral genes unessential for replication in tumor cells.

Onyx-15 is the first adenoviral vector in clinical trials for the treatment of glioma [84]. This vector lacks E1B-55kD gene, a gene thought initially to function by degrading cellular p53 therefore making its replication specific for transformed cells that lack p53 activity [85]. However subsequent studies demonstrated that the most likely explanation for its oncolytic mechanism involves inhibition of intracellular RNA transport [86, 87]. A number of clinical trials for glioma have been carried out with AdVs. Phase I studies 
Table 1-3. Current oncolytic viral therapies in clinical trials for the treatment of GBM

\begin{tabular}{|c|c|c|c|c|c|c|c|}
\hline $\begin{array}{c}\text { Virus } \\
\text { (Vector) }\end{array}$ & Characteristics & $\begin{array}{c}\text { Oncolytic } \\
\text { Mechanism: } \\
\text { Viral Factor/ } \\
\text { Host Target }\end{array}$ & $\begin{array}{c}\text { Clinical } \\
\text { Trials.gov } \\
\text { ID }^{\mathbf{a}}\end{array}$ & $\begin{array}{l}\text { Delivery } \\
\text { Method }^{b}\end{array}$ & $\begin{array}{c}\text { Phase Trial- } \\
\text { Status }\end{array}$ & Outcomes $^{c}$ & References \\
\hline $\begin{array}{l}\text { Adenovirus } \\
(\Delta 24-R G D)\end{array}$ & $\begin{array}{l}\text { Family: } \\
\text { Adenoviridae } \\
\text { Structure: Non- } \\
\text { enveloped } \\
\text { linear dsDNA }\end{array}$ & $\begin{array}{l}\text { E1A deletion/ } \\
\text { pRB dys- } \\
\text { regulation } \\
\text { Fiber knob } \rightarrow \text { RGD } \\
\text { motif/ CAR } \\
\text { under-expression }\end{array}$ & $\begin{array}{c}\text { NCT- } \\
01582516\end{array}$ & CED & $\begin{array}{l}\text { Phase I- } \\
\text { recruiting }\end{array}$ & $\begin{array}{l}\text { Safety of } \\
\text { intervention in } \\
\text { recurrent GBM; } \\
\text { PFS, OS at } 6 \\
\text { months }\end{array}$ & [88-90] \\
\hline $\begin{array}{l}\text { Adenovirus } \\
\text { (Onyx-015) }\end{array}$ & $\begin{array}{l}\text { Family: } \\
\text { Adenoviridae } \\
\text { Structure: Non- } \\
\text { enveloped } \\
\text { linear dsDNA }\end{array}$ & $\begin{array}{l}\text { E1B-55kDa } \\
\text { deletion/p53 dys- } \\
\text { regulation and/or } \\
\text { RNA transport }\end{array}$ & $\mathrm{N} / \mathrm{A}$ & i.t. & $\begin{array}{l}\text { Phase I- } \\
\text { complete }\end{array}$ & $\begin{array}{l}\text { Intervention } \\
\text { safe at MTD; } \\
\text { Mild antitumor } \\
\text { effect; } \\
4.9 \text { mo median } \\
\text { survival }\end{array}$ & {$[84-87,91]$} \\
\hline $\begin{array}{l}\text { Herpes } \\
\text { simplex } \\
\text { virus } \\
\text { (HSV1716) }\end{array}$ & $\begin{array}{l}\text { Family: } \\
\text { Herpesviridae } \\
\text { Structure: } \\
\text { Enveloped } \\
\text { linear dsDNA }\end{array}$ & $\begin{array}{l}\text { Deletion in } \\
\text { ICP34.5 gene/ } \\
\text { eIF2a regulated } \\
\text { protein synthesis }\end{array}$ & N/A & $\begin{array}{l}\text { i.c. post- } \\
\text { resection }\end{array}$ & $\begin{array}{l}\text { Phase I- } \\
\text { complete } \\
\text { Phase II- } \\
\text { recruiting }\end{array}$ & $\begin{array}{l}\text { Phase I: } \\
\text { Intervention } \\
\text { safe at MTD; } \\
3 \text { patients with } \\
\text { PFS of } 3 \text { years; } \\
\text { recovered virus } \\
\text { from tumor } \\
\text { Phase II: } \\
\text { Efficacy in adult } \\
\text { with recurrent } \\
\text { GBM }\end{array}$ & [92-95] \\
\hline
\end{tabular}


Table 1-3. Continued

\begin{tabular}{|c|c|c|c|c|c|c|c|}
\hline $\begin{array}{c}\text { Virus } \\
\text { (Vector) }\end{array}$ & Characteristics & $\begin{array}{c}\text { Oncolytic } \\
\text { Mechanism: } \\
\text { Viral Factor/ } \\
\text { Host Target }\end{array}$ & $\begin{array}{c}\text { Clinical } \\
\text { Trials.gov } \\
\text { ID }^{\mathrm{a}}\end{array}$ & $\begin{array}{l}\text { Delivery } \\
\text { Method }^{\text {b }}\end{array}$ & $\begin{array}{c}\text { Phase Trial- } \\
\text { Status }\end{array}$ & Outcomes $^{c}$ & References \\
\hline $\begin{array}{l}\text { Herpes } \\
\text { simplex } \\
\text { virus } \\
(\mathrm{G} 207)\end{array}$ & $\begin{array}{l}\text { Family: } \\
\text { Herpesviridae } \\
\text { Structure: } \\
\text { enveloped } \\
\text { linear dsDNA }\end{array}$ & $\begin{array}{l}\text { ICP34.5 deletion/ } \\
\text { eIF2 } \alpha \text { regulated } \\
\text { protein synthesis; } \\
\text { UL39 replaced } \\
\text { with lacZ reporter/ } \\
\text { decreased neuro- } \\
\text { toxicity and } \\
\text { increased } \\
\text { sensitivity to } \\
\text { antivirals }\end{array}$ & $\begin{array}{c}\text { NCT- } \\
00028158 \\
\text { NCT- } \\
00157703 \\
\text { (with } \\
\text { radiation) }\end{array}$ & $\begin{array}{l}\text { i.t and i.c } \\
\text { post- } \\
\text { resection }\end{array}$ & $\begin{array}{l}\text { Phase I/II- } \\
\text { complete }\end{array}$ & $\begin{array}{l}\text { Phase I: } \\
\text { Intervention } \\
\text { was safe at } \\
\text { MTD; modest } \\
\text { increase in OS, } \\
\text { PFS; virus } \\
\text { recovered from } \\
\text { tumor } \\
\text { Phase I/II: } \\
\text { Safety and } \\
\text { effectiveness of } \\
\text { intervention } \\
\text { with radiation }\end{array}$ & [96-101] \\
\hline $\begin{array}{l}\text { Parvovirus } \\
\text { (ParvOryx } \\
\text { or H-1PV) }\end{array}$ & $\begin{array}{l}\text { Family: } \\
\text { Parvoviridae } \\
\text { Structure: non- } \\
\text { enveloped, } \\
\text { ssDNA }\end{array}$ & $\begin{array}{l}\text { Naturally } \\
\text { occurring/ IFN } \\
\text { defects }\end{array}$ & $\begin{array}{c}\text { NCT- } \\
01301430\end{array}$ & $\begin{array}{l}\text { i.t }+ \text { i.c. post- } \\
\text { resection } \\
\text { versus i.v }+ \\
\text { i.c. post- } \\
\text { resection }\end{array}$ & $\begin{array}{c}\text { Phase I/IIa- } \\
\text { recruiting }\end{array}$ & $\begin{array}{l}\text { Safety and } \\
\text { efficacy of } \\
\text { intervention in } \\
\text { progressive } \\
\text { primary or } \\
\text { recurrent GBM; } \\
\text { PFS, OS, and } \\
\text { viral replication } \\
\text { in tumor }\end{array}$ & [102-107] \\
\hline
\end{tabular}


Table 1-3. Continued

\begin{tabular}{|c|c|c|c|c|c|c|c|}
\hline $\begin{array}{c}\text { Virus } \\
\text { (Vector) }\end{array}$ & Characteristics & $\begin{array}{c}\text { Oncolytic } \\
\text { Mechanism: } \\
\text { Viral Factor/ } \\
\text { Host Target }\end{array}$ & $\begin{array}{c}\text { Clinical } \\
\text { Trials.gov } \\
\text { ID }^{\mathrm{a}}\end{array}$ & $\begin{array}{l}\text { Delivery } \\
\text { Method }^{b}\end{array}$ & $\begin{array}{c}\text { Phase Trial- } \\
\text { Status }\end{array}$ & Outcomes $^{c}$ & References \\
\hline $\begin{array}{l}\text { Newcastle } \\
\text { disease virus } \\
(\mathrm{NDV}-\mathrm{HUJ})\end{array}$ & $\begin{array}{l}\text { Family: } \\
\text { Paramyxo- } \\
\text { viridae } \\
\text { Structure: } \\
\text { Enveloped } \\
\text { negative ssRNA }\end{array}$ & $\begin{array}{l}\text { Naturally } \\
\text { occurring/ } \\
\text { IFN defects; } \\
\text { PKR and } \\
\text { MxA } \\
\text { deficiency }\end{array}$ & $\begin{array}{c}\text { NCT- } \\
01174537\end{array}$ & i.v. & $\begin{array}{c}\text { Phase I/II- Not } \\
\text { yet recruiting }\end{array}$ & $\begin{array}{l}\text { Phase I: } \\
\text { Intervention } \\
\text { was safe at } \\
\text { MTD in } \\
\text { recurrent } \\
\text { GBM; 1 } \\
\text { permanent } \\
\text { regression } \\
\text { and } 2 \text { long } \\
\text { term } \\
\text { survivors } \\
\text { Phase II: PFS } \\
\text { in GBM }\end{array}$ & [108-111] \\
\hline $\begin{array}{l}\text { Reovirus } \\
\text { (Reolysin) }\end{array}$ & $\begin{array}{l}\text { Family: } \\
\text { Reoviridae } \\
\text { Structure: } \\
\text { Non-enveloped } \\
\text { segmented } \\
\text { dsRNA }\end{array}$ & $\begin{array}{l}\text { Naturally } \\
\text { occurring/ } \\
\text { overactive } \\
\text { Ras pathway }\end{array}$ & $\begin{array}{c}\text { NCT- } \\
00528684\end{array}$ & i.t. & $\begin{array}{l}\text { Phase I/II- } \\
\text { complete }\end{array}$ & $\begin{array}{l}\text { Phase I: } \\
\text { Intervention } \\
\text { was safe at } \\
\text { MTD in } \\
\text { refractory } \\
\text { GBM; } 1 \text { long } \\
\text { term survivor } \\
\text { at } 6 \text { years } \\
\text { Phase II: } \\
\text { results } \\
\text { pending }\end{array}$ & [112-117] \\
\hline
\end{tabular}


Table 1-3. Continued

\begin{tabular}{|c|c|c|c|c|c|c|c|}
\hline $\begin{array}{c}\text { Virus } \\
\text { (Vector) }\end{array}$ & Characteristics & $\begin{array}{c}\text { Oncolytic } \\
\text { Mechanism: } \\
\text { Viral Factor/ } \\
\text { Host Target }\end{array}$ & $\begin{array}{c}\text { Clinical } \\
\text { Trials.gov } \\
\text { ID }^{\mathbf{a}}\end{array}$ & $\begin{array}{l}\text { Delivery } \\
\text { Method }^{\mathrm{b}}\end{array}$ & $\begin{array}{c}\text { Phase Trial- } \\
\text { Status }\end{array}$ & Outcomes $^{c}$ & References \\
\hline $\begin{array}{l}\text { Measles virus } \\
\text { (MV-CEA) }\end{array}$ & $\begin{array}{l}\text { Family: } \\
\text { Paramyxo- } \\
\text { viridae } \\
\text { Structure: } \\
\text { Enveloped } \\
\text { negative ssRNA }\end{array}$ & $\begin{array}{l}\text { H protein of } \\
\text { vaccine strain/ } \\
\text { CD46 over- } \\
\text { expression } \\
\text { P and V } \\
\text { proteins/ } \\
\text { Defective IFN } \\
\text { pathway }\end{array}$ & $\begin{array}{c}\text { NCT- } \\
00390299\end{array}$ & $\begin{array}{l}\text { i.c post- } \\
\text { resection only } \\
\text { versus i.t }+ \text { i.c } \\
\text { post-resection }\end{array}$ & $\begin{array}{l}\text { Phase I/II- } \\
\text { complete }\end{array}$ & $\begin{array}{l}\text { Safety, PFS, } \\
\text { and antiviral } \\
\text { responses in } \\
\text { recurrent } \\
\text { glioma }\end{array}$ & [118-123] \\
\hline $\begin{array}{l}\text { Poliovirus } \\
\text { (PVSRIPO) }\end{array}$ & $\begin{array}{l}\text { Family: } \\
\text { Picornaviridae } \\
\text { Structure: Non- } \\
\text { enveloped } \\
\text { positive ssRNA }\end{array}$ & $\begin{array}{l}\text { Polio IRES } \rightarrow \\
\text { rhinovirus } \\
\text { IRES/ } \\
\text { decreased } \\
\text { neuro-toxicity } \\
\text { Necl-5 } \\
\text { (CD155) over- } \\
\text { expression }\end{array}$ & $\begin{array}{c}\text { NCT- } \\
01491893\end{array}$ & CED & $\begin{array}{l}\text { Phase I- } \\
\text { recruiting }\end{array}$ & $\begin{array}{l}\text { Safety at } \\
\text { MTD in } \\
\text { recurrent } \\
\text { GBM; PFS } \\
\text { and OS }\end{array}$ & [124-131] \\
\hline
\end{tabular}


Table 1-3. Continued

\begin{tabular}{|c|c|c|c|c|c|c|c|}
\hline $\begin{array}{c}\text { Virus } \\
\text { (Vector) }\end{array}$ & Characteristics & $\begin{array}{c}\text { Oncolytic } \\
\text { Mechanism: } \\
\text { Viral Factor/ } \\
\text { Host Target }\end{array}$ & $\begin{array}{c}\text { Clinical } \\
\text { Trials.gov } \\
\text { ID }^{\mathrm{a}}\end{array}$ & $\begin{array}{l}\text { Delivery } \\
\text { Method }^{b}\end{array}$ & $\begin{array}{c}\text { Phase Trial- } \\
\text { Status }\end{array}$ & Outcomes $^{\mathrm{c}}$ & References \\
\hline $\begin{array}{l}\text { Retrovirus } \\
\text { (TOCA 511) }\end{array}$ & $\begin{array}{l}\text { Family: } \\
\text { Retroviridae } \\
\text { Structure: } \\
\text { enveloped } \\
\text { ssRNA }\end{array}$ & $\begin{array}{l}\text { Naturally } \\
\text { occurring/ } \\
\text { antiviral } \\
\text { defects } \\
\text { Cytosine } \\
\text { deaminase } \\
\text { insertion } \\
(5 \mathrm{FC} \rightarrow 5 \mathrm{FU})\end{array}$ & $\begin{array}{c}\text { NCT- } \\
01156584 \\
\text { NCT- } \\
01470794\end{array}$ & $\begin{array}{l}\text { i.t. versus i.c. } \\
\text { post-resection }\end{array}$ & $\begin{array}{c}\text { Phase I- } \\
\text { recruiting }\end{array}$ & $\begin{array}{l}\text { Safety at } \\
\text { MTD and } \\
\text { efficacy in } \\
\text { recurrent } \\
\text { GBM; PFS at } \\
6 \text { months }\end{array}$ & $\begin{array}{c}{[132-136][50,} \\
137]\end{array}$ \\
\hline
\end{tabular}

\footnotetext{
${ }^{a}$ Keyword search on clinicaltrials.gov included glioma, glioblastoma, brain cancer, virus, oncolytic virus

${ }^{\mathbf{b}} \mathrm{CED}$, convection enhanced delivery; i.t, intratumoral; i.v, intravenous; i.c, intracranial

'PFS, progression free survival; OS, overall survival; MTD, maximum tolerated dose
} 
with Onyx-15 as adjuvant treatment for recurrent glioblastoma reported the virus to be well tolerated and safe at the maximum dose, however very little antitumor effect was observed with a median survival time of 4.9 months similar to the median survival of untreated patients (reviewed in [138] and [65]) [91]. There have been no additional clinical trials scheduled using Onyx-015 for GBM. However a modified version of the Onyx-015, H101, has completed Phase III trials and was approved in 2005 as an adjuvant therapy for the treatment of head and neck cancers in China. H101, therefore, is the first oncolytic virus to be marketed for clinical use worldwide [139].

$\triangle 24-\mathrm{RGD}$ is another CRAd being studied as a potential therapy for GBM. It is a genetically engineered vector that harbors a partial deletion of 24 base pairs in its E1A gene. This deletion decreases its ability to bind to $\mathrm{pRb}$ so replication is permitted in cells with $\mathrm{pRb}$ defects. Coxsackie adenovirus receptor (CAR), is a well known cellular receptor of many AdV serotypes and its expression has been found to be very low on tumor cells including glioma [140-142]. In order to bypass the need to interact with $\mathrm{CAR}$, an alternative binding strategy used by a number of adenoviruses was inserted into the $\Delta 24-$ RGD. The addition of the Arg-Gly-Asp (RGD) motif, known to interact with cellular $\alpha_{v}$ integrins, into the adenovirus fiber knob allowed this CrAd to maintain tropism in glioma cells in a CAR-independent fashion [88, 143]. In vivo testing of human glioma xenografts treated with $\Delta 24$-RGD showed complete tumor regression in 9 of 10 treated mice and increased survival in all treated mice. These results were even more pronounced when viral treatment was preceded by tumor irradiation [89]. A phase I trial studying $\triangle 24-R G D$ in patients with recurrent glioma using convection enhanced delivery is ongoing [90].

\section{Herpes simplex virus}

HSV is an enveloped, linear dsDNA virus of the family, Herpesviridae. Primary infection with HSV ranges from asymptomatic to gingivostomatitis. It is known that HSV possesses a neurotropic phenotype, therefore, after initial infection, virus migrates through nerve endings to cranial ganglia where it remains latent. Recurrent infections can manifest in a number of ways from perioral "fever" blisters, conjunctivitis, and Bell's palsy, to more serious infections such as encephalitis. HSV prevalence is high, with $\sim 90 \%$ of the population demonstrating seroconversion [144].

There are several advantages to using HSV as an oncolytic therapy. It has been shown that HSV genes associated with neurovirulence are different from those having oncolytic properties which provides a means of genetic manipulation to create more oncolytic but less neurotropic viral vectors. In addition, because of its sensitivity to antiviral drugs such as VCV and GCV, a naturally built-in safety mechanism can be used as precaution for uncontrolled HSV infection. Several disadvantages of HSV oncolysis include low production of viral progeny per cell as well as the high prevalence of previous infection in the adult population (reviewed in [83]).

The first example of an HSV oncolytic virus is HSV1716. It is a null mutant of 
the gene coding for $\gamma 34.5$ (ICP34.5), a virulence factor whose absence results in selective replication in dividing cells [92]. During viral infection, products of gene replication are sensed by double-stranded RNA- dependent protein kinase R (PKR) that, in turn, phosphorylates eIF2 into its active form, serving as an off switch of cellular protein synthesis. ICP34.5 deactivates eIF2 by encouraging its dephosphorylation therefore allowing protein synthesis to continue. Without ICP34.5, normal cells can stop protein synthesis in the presence of viral infection. Cells harboring defects in PKR or PKR related signals, such as the case with ras deregulated tumor cells, have unopposed protein synthesis in the presence of virus, allowing tumor selective replication [93, 145]. Several clinical trials have been carried out using HSV1716 including those looking at safety of intratumoral (i.t.) injection pre-resection and intracranial injection post resection. All trials demonstrated safety at the maximum dose without signs of neurotoxicity, a major concern with HSV related therapies. Additionally, three patients experienced over 3 years PFS and biological endpoints confirmed replication in tumor samples post-injection [94]. Phase II studies are currently being prepared in Europe using HSV1716 (Seprehvir) however no results have been reported to date [95].

Another HSV-based OV currently being evaluated in clinical trials is G207. This virus harbors two separate genetic alterations, one being a deletion in the ICP34.5 coding gene, RL1. The other alteration includes the silencing of the UL39 gene by insertion of LacZ. UL39 is a gene that encodes a subunit of the viral ribonucleotide reductase (ICP6). Inactivation of this gene not only increases HSV susceptibility to antivirals such as GCV and VCV but also dampens its neurotropic phenotype, resulting in a virus unable to cause encephalitis. Alterations in this gene, therefore, improve the safety profile of G207 over wildtype [96-98]. Previous phase I trials tested G207 by administering therapy in 2 stages, $13 \%$ of dose was given by stereotactic injection and the remainder several days later at time of resection. Results confirmed G207 to be safe with no signs of neurotoxicity at maximum dose. Median time to progression was 3 months, with median overall survival of 23 months post-diagnosis, approximately 7 months after viral inoculation. One patient experienced more than 5 years PFS, and this patient was found to have the highest viral load present in tumor samples collected at resection [99, 100, 146]. Another Phase I trial was completed to determine synergistic effects of intratumoral G207 with either focal radiation or gamma knife surgery. No results have been published to date [101].

\section{Parvovirus}

Another DNA virus, newer in the clinical trial network for GBM, is parvovirus (Parvo), a small non-enveloped single-stranded DNA rodent virus surrounded by an icosahedral capsid. It is a member of the Parvoviridae family and represents a group of autologous parvoviruses that are able to replicate in and lyse tumor cells by several mechanisms. First, they are able to replicate more efficiently in transformed cells as these cells, with their high proliferative rate, provide active factors important in DNA synthesis and transcriptional machinery. These factors are crucial in the processing of Parvo replicative forms $(\mathrm{RF})$, which are dsDNA formed during self primed DNA 
synthesis and contain both parental and newly synthesized DNA strands [102, 147]. Parvo is also able to inhibit IFN responses of transformed cells even after exposure to IFN inducers such as polyI:C by preventing the activation of specific pattern recognition receptors [103]. Advantages of oncolytic parvo include its nonpathogenic phenotype in humans, usually only causing asymptomatic infection, unlike its relative, parvovirus B19. It has been shown to elicit viral replication in brain tumors following systemic administration, and replication is restricted to proliferating tissues. On the other hand, difficulty of producing large quantities of parvovirus by co-transfection in helper-free virus-producing cells along with its immaturity in clinical trials threaten to limit its oncolytic potential (reviewed in [148]).

The most studied parvovirus for treatment of glioma includes the naturally occurring virus, H-1PV (ParvOryx). Preclinical studies have demonstrated its ability to infect glioma cells with strong cytotoxic effects $[105,149]$. In vivo experiments using a rat glioma model, following either a single i.t. injection or multiple i.v. injections of ParvOryx, results showed tumor regression, a significant increase in survival, and evidence of viral presence in brain tumors confirming its ability to cross the BBB after systemic administration [106]. An ongoing Phase I/II trial of ParvOryx in patients with progressive primary or recurrent tumors is coming to a close and has been focused on determining safety and MTD in groups receiving either an initial i.t. or i.v. injection both followed by injection to post-resection tumor bed. These results will be the first of any trial evaluating a parvovirus in patients with recurrent GBM [107].

\section{Newcastle disease virus}

In addition to DNA viruses, there are a number of RNA viruses that are being considered as treatment for GBM and are currently in varying stages of clinical trials. These include both naturally occurring and genetically engineered RNA viruses. Newcastle Disease virus (NDV) is an avian paramyxovirus of the order, Mononegavirales, therefore it characteristically contains a negative-sense ssRNA genome which codes for pleiomorphic viral particles. Though NDV is primarily a disease-causing agent of fowl, it has a broad host range and has been shown to infect cells of other species including murine and human cells [150]. Two naturally occurring, nonengineered strains of NDV have been studied in Phase I clinical trials for glioma, NDVHUJ which is non-pathogenic in avian hosts (lentogenic), and the moderately pathogenic (mesogenic) MTH68/H strain. Their mechanism of oncolysis has been attributed to the ability to exploit interferon defects in tumor cells (reviewed in [63]). Several studies have been geared toward improving their oncolytic activity through genetic manipulation of NDVs anti-IFN genes allowing for robust IFN production in normal cells leaving IFN defective tumor cells vulnerable to viral infection [108].

Advantages of NDV oncolytic therapy include its safety profile when given intravenously. Because its natural host is avian, there is no pre-established immunity to virus in humans. NDV is a naturally occurring oncolytic virus and produces large numbers of progeny per infected cell. Disadvantages to therapy include the risk of species 
adaptation and its high mutation rate, a characteristic of all RNA viruses [83]. Phase I trials have been completed using the NDV-HUJ strain in patients with recurrent GBM. NDV-HUJ is a gradient purified virus derived from an attenuated vaccine strain [109]. Patients received daily i.v. injections, 5 days/week until one year of tumor progression was observed. Results showed virus to be well-tolerated with no signs of toxicity other than transient fever associated with infection. One patient displayed complete but not permanent regression while this patient and 2 others experienced long term survival, living approximately 15 months with recurrent disease after viral treatment $[109,138]$. A phase II trial of NDV-HUJ in recurrent glioma is ongoing and has extended the use of oncolytic treatment to sarcoma and neuroblastoma patients as well [111].

\section{Reovirus}

Reovirus is a non-enveloped virus with a segmented, dsRNA genome. It is of the family, Reoviridae, along with its relative, rotavirus, an important enteric virus causing substantial disease of the gastrointestinal tract in humans. Reovirus, on the other hand, causes milder GI symptoms, which usually resolve without intervention [151]. It is a naturally occurring oncolytic virus whose oncolytic activity stems from its ability to exploit cells with overactive ras or ras- signaling $[112,113]$. In many cancer cells, upregulation of ras confers a resistance to cell death; however, this results in inhibition of active PKR, responsible for cellular protein shut off during viral infection [64, 112]. Advantages of reoviral therapy include its safe profile when administered in the brain [114], its naturally occurring anti tumor activity through cell specific mutations to ras signaling, its high progeny: cell ratio, and the fact that all genes in the viral genome have known functions. Disadvantages to therapy include pre-immunity in the human population and its high rate of mutation during replication cycles (reviewed in [83]) [115]).

In preclinical studies, reovirus was found to inhibit growth of ipsilateral tumors in immunocompetent glioma rat models despite the presence of pre-existing immunity. Virus safety was also confirmed in non-human primates by the lack of significant toxicity following intracranial inoculation [114]. Reolysin, the non-attenuated Type 3 Dearing strain of reovirus, has been tested as an adjuvant therapy in phase I trials at doses ranging from $10^{7}-10^{10}$ in patients with refractory GBM. Results showed treatment to be well tolerated and safe at the maximum dose delivered. Median survival was 21 weeks with one patient still living at six years post treatment according to latest updates [116, 138]. Phase II of this series has been completed using the highest dose reached from phase I however no results have been published to date [117].

\section{Measles virus}

Measles virus (MV) is an enveloped, negative strand RNA virus belonging to the Morbillivirus genus of the family, Paramyxoviridae. It is known to be a highly infectious human pathogen transmitted by respiratory droplets from infected individuals. Flu-like 
symptoms usually occur within 10 days and progress to the characteristic maculopapular rash. Small white lesions called Koplik's spots can often be seen on the buccal mucosa before the development of rash [152]. With the advent of vaccination programs for prevention of the spread of measles, endemic transmission has been eradicated from the Americas since 2002 though outbreaks continue to occur worldwide [153, 154].

The replication cycle of measles involves binding of viral haemagglutinin $(\mathrm{H})$ protein to a predominantly B and T lymphocyte receptor, SLAM (signaling lymphocyticactivation molecule) [118]. Attenuated forms of MV such as that derived from the Edmonston B vaccine, predominantly bind to CD46, a complement-regulatory protein [119] important in the protection of cells against complement-mediated lysis. Cells independent of SLAM and CD46 signaling, specifically those of epithelial origin, have been shown to use Nectin4 for receptor activity during MV infection [155, 156]. As such, both CD46 and Nectin4 are often overexpressed in tumors, with CD46 shown to be overexpressed in glioma [157-161]. By targeting tumor specific overexpression of MV specific receptors, measles virus can be considered a naturally occurring oncolytic virus. MV-CEA is an example of an attenuated strain, which has been genetically engineered to express human carcinoembryonic antigen (CEA). CEA is a tumor marker highly expressed in cancers ranging from colorectal to pancreatic carcinomas, however CEA overexpression has not been observed in gliomas. For this reason, its presence in sera of patients infected with the MV-CEA construct serves as a monitor of viral gene expression [120]. Advantages of measles therapy include its wide host range and ability of attenuated virus to selectively replicate in tumor cells based on naturally acquired mutations in the viral attachment protein [115]. Attenuated strains are considered to have preferred safety profiles based on the extensive history of their use in vaccination programs that have improved morbidity and mortality worldwide [83]. On the other hand, despite altered attachment proteins, virus may still be able to bind to CD46 and SLAM on normal cells. This serves as a disadvantage in using measles virus as a cancer therapy since SLAM signaling during measles infection has been shown to facilitate transient immunosuppression [162]. In addition, much of the population has been vaccinated against the virus therefore strong immune responses directed against the $\mathrm{H}$ protein may be experienced [152]. Lastly, as with replication of most RNA viruses, measles has a high mutation rate due to the error-prone RNA-dependent RNA polymerase that could potentially alter selectivity during treatment.

In vitro experiments with MV-CEA have demonstrated strong antitumor effects against glioma cells, which have translated into antitumor activity in subcutaneous and orthotopic U87 glioma animal models. Further in vivo studies of MV-CEA in an orthotopic glioma model in macaque monkeys found the vector to be safe, causing no neurotoxicity when given intracranially [122]. An ongoing phase I trial is evaluating safety and MTD of MV-CEA following i.t. injection versus i.t. injection administered post-resection in patients with recurrent glioblastoma. There have been no reported preliminary results however the study will tentatively be completed by June 2013 [123]. 


\section{Poliovirus}

Another RNA virus being considered as therapy for GBM is poliovirus, a pathogen notorious for causing disease worldwide before the launch of a global initiative in the 1980's resulted in its eradication from the Americas in 2002 [163]. Poliovirus (polio) is a non-enveloped +ssRNA enterovirus belonging to the family, Picornaviridae [164]. Polio is transmitted by fecal-oral route and usually causes asymptomatic infection in the majority of cases. In instances when virus is not cleared, polio can spread through the blood to other sites including the CNS. Because polio is naturally neurovirulent, destruction of motor neurons can cause poliomyelitis manifesting as flaccid paralysis that may lead to death if control of respiratory function is affected [124]. As an RNA virus, the replication cycle occurs exclusively in the cytoplasm of host cells. Polio genome characteristically includes an important segment of RNA known as the internal ribosome entry site (IRES) from where one open reading frame initiates coding of a large precursor polypeptide that is further cleaved into the virus's eleven proteins $[115,164]$.

There are several important components to polio's oncolytic activity, the first involving its cellular binding receptor, CD155 (or Necl-5) [124]. Necl-5 has been shown to be over-expressed in a number of tumor cells including cancers of glial, breast, and hepatocellular origin $[125,165]$. The other component of polio's oncolytic activity involves the manner in which the virus is able to recruit host cell machinery for its benefit. Polio uses a 5'cap independent protein translation mechanism so during its replication cycle, the virus must seize host cell machinery in order to form new viral progeny. This is done through the actions of 2A protease (2Apro), which is able to cleave the scaffold (eIF4G) connecting eukaryotic mRNA 5'cap to ribosomes. These cleaved scaffold fragments are then free to aid in linking the polio IRES to cellular ribosomes for its own translational benefit. This takeover subsequently leads to inhibition of translational dependent antiviral responses and rapid cytopathic effects can be detected as soon as 2-3 hours post-infection [126].

Many cancers with their increased need for protein synthesis in order to maintain their uninhibited growth have been shown to have cap-independent translation mechanisms [166]. Along with this independence, some tumor cells appear to have less control over recruitment of ribosomes to viral RNA as a number of cellular proteins involved in seizing viral genomes are mislocated to the nucleus and not in the cytoplasm where viral replication actually occurs [167]. In addition, several studies have shown that inhibitors of many deregulated mitogen pathways in cancers such as EGFR, which are known to be interconnected with eIF4G, decrease poliovirus translation and cytotoxicity in GBM cells [168]. This serves as another example of how deregulation of pathways during tumorigenesis affects susceptibility to viral infection.

The construct used in preclinical and clinical studies is the PVSRIPO. In order to minimize neurovirulence in normal CNS, the poliovirus IRES is exchanged for the IRES present in human rhinovirus type 2 (HRV2), a known pathogen of the human respiratory tract. The resulting construct is a polio/rhinovirus chimera known as RIPO. PVSRIPO was derived from using an attenuated Serotype 1 Sabin vaccine poliovirus instead of 
wildtype virus $[128,169]$. Advantages to poliovirus cancer therapy include its ability to be easily engineered especially in manipulating genes known to contribute to neurovirulence. The neuro-attenuated construct, PVSRIPO, quickly kills cells with accelerated growth rates such as transformed cells [129]. Disadvantages include the extensive patient population that have already been immunized against polio however preclinical studies have revealed that oncolysis is not decreased by pre-existing immunity in animal models $[124,130]$. Also, its error-prone RNA-dependent RNA polymerase has been shown to cause revertants implicated in poliomyelitis following vaccination. By using the PVSRIPO construct with replacement of polio IRES, the known target for mutation in revertants, the chances of this occurring is unlikely [170]. A phase I trial is currently evaluating the use of PVSRIPO administered by CED in patients with recurrent glioma. Outcomes to be determined include safety, maximum tolerated dose, progression-free survival and overall survival [131].

\section{Retrovirus}

The last virus being considered in clinical trials for the treatment of GBM are retroviruses, which are enveloped ssRNA virus of the family Retroviridae. This family of viruses has until recently been a less popular alternative for replicating viral therapy against brain tumors as it defies several important characteristics of other OVs. First, an important feature of retroviruses, particularly replicating retroviral vectors (RVVs) is their unique replication cycle. In order for replication of the RNA genome to occur, the retrovirus carries with it a reverse transcriptase that synthesizes a DNA replication intermediate from its RNA genome. The intermediate is integrated into the host cell genome for subsequent transcription and translation using the host cell machinery. A property of oncolytic viruses, as the name implies, is their ability to lyse tumor cells. RVVs however are non-lytic, a direct result of their need to integrate into the host genome (reviewed in [83]). Integration is also known to be an important feature of retroviral-induced tumorigenesis.

Approximately $11 \%$ of human cancers are caused by viral infections. Malignancies, such as lymphoma and sarcoma, have been associated specifically with retroviral infection [171]. Insertional mutagenesis has also proven to be an issue in previous gene therapy trials using replication defective retroviral vectors. For instance, clinical trials for several X-linked immune diseases such as Severe Combined Immunodeficiency (SCID) and Wiskott-Aldrich Syndrome (WAS) resulted in development of acute T lymphoblastic leukemia (T-ALL) after DNA intermediates integrated into the host genome in close proximity to proto-oncogenes [132, 172-174]. Therefore, the use of these viruses to treat cancer may appear to be more of a risk than a benefit.

As we know, however, there are a number of advantages to retroviral therapy. First, RVV constructs, particularly those being considered for treatment of glioma, have two mechanisms of targeting tumor cells; they are selective for dividing cells as they do not contain nuclear localization signals necessary for active nuclear uptake in quiescent 
cells $[133,175]$ and they selectively replicate in cells harboring defects in their antiviral responses. RVVs are less immunogenic in the CNS where virus-infected cells have been shown to escape antiviral responses [176]. As a consequence of integration into the host genome, RVVs maintain a persistent infection in tumor cells, which act as a storage for virus that can be called upon to replicate at later times $[83,134,175]$.

The most studied retrovirus for treatment of cancer is the simple gammaretrovirus, amphotropic murine leukemia virus (A-MLV). Derived from this prototypical retrovirus is the construct Toca511, which has been genetically engineered to express the yeast enzyme, cytosine deaminase (CD) that converts the anticancer pro-drug 5-fluorocytosine (5-FC) to its active form, 5-fluorouracil (5-FU). Toca511 is the most used construct in preclinical and clinical trials for GBM and is a great example of an $\mathrm{OV}$ that functions by a suicide gene/pro-drug combination $[56,135]$. Preclinical studies using an orthotopic mouse GBM xenograft model demonstrated survival of $90 \%$ of treated mice at $\sim 100$ days post-infection versus 40 days in controls [133]. In two different syngeneic immunocompetent mouse GBM models (CT26 in BALB/c mice and Tu-2449 in B6C3F1 mice), survival was significantly increased in BALB/c mice from 30.5 days in controls to $>90$ days and from 33 days to $>180$ days in B6C3F1 mice. Subsequent rounds of 5-FC pro-drug prolonged survival even without having to administer more retrovirus proving its stability after incorporation into the host genome. Even before the last cycle of pro-drug, tumors shrank until they were no longer detected microscopically [134]. Until recently, only one phase trial has been performed using a retrovirus for treatment of GBM. This phase III trial, unfortunately, was unsuccessful most likely due to the use of a replication defective construct [136]. There are two ongoing phase I trials evaluating replication competent Toca 511 for the treatment of recurrent high-grade glioma using either intratumoral injection followed by administration of 5-FC [50] or post-resection injection followed by 5-FC [137]. The study is planned to complete in December of 2013.

\section{Future of Oncolytic Therapy: Challenges to Overcome}

As more oncolytic viruses are considered for use in cancer therapy, several important challenges in achieving optimal efficacy will need to be addressed as they have proven to be a barrier in moving therapies through the clinical trial pipeline (Figure 1-3). The first challenge is that of finding the most effective delivery of treatment in order to maintain tumor cytotoxicity. This challenge is not necessarily unique to OV therapy, as a number of problems exist with the administration of chemotherapeutic agents, which have led to more experimental methods for administering treatment such as CED or polymer wafers. One method of delivery for OV therapy includes single or multiple intratumoral injections. Tissue analysis following injection often shows very little spread of virus within the tumor mass with most virus concentrated near the injection site [177]. Aside from high interstitial pressure exerted by tumor mass [178], the unique tumor microenvironment often plays a significant role in impeding the spread of virus. This microenvironment is composed not only of surrounding tumor, normal brain, and patrolling inflammatory cells but also players in promoting angiogenesis, growth factors, 


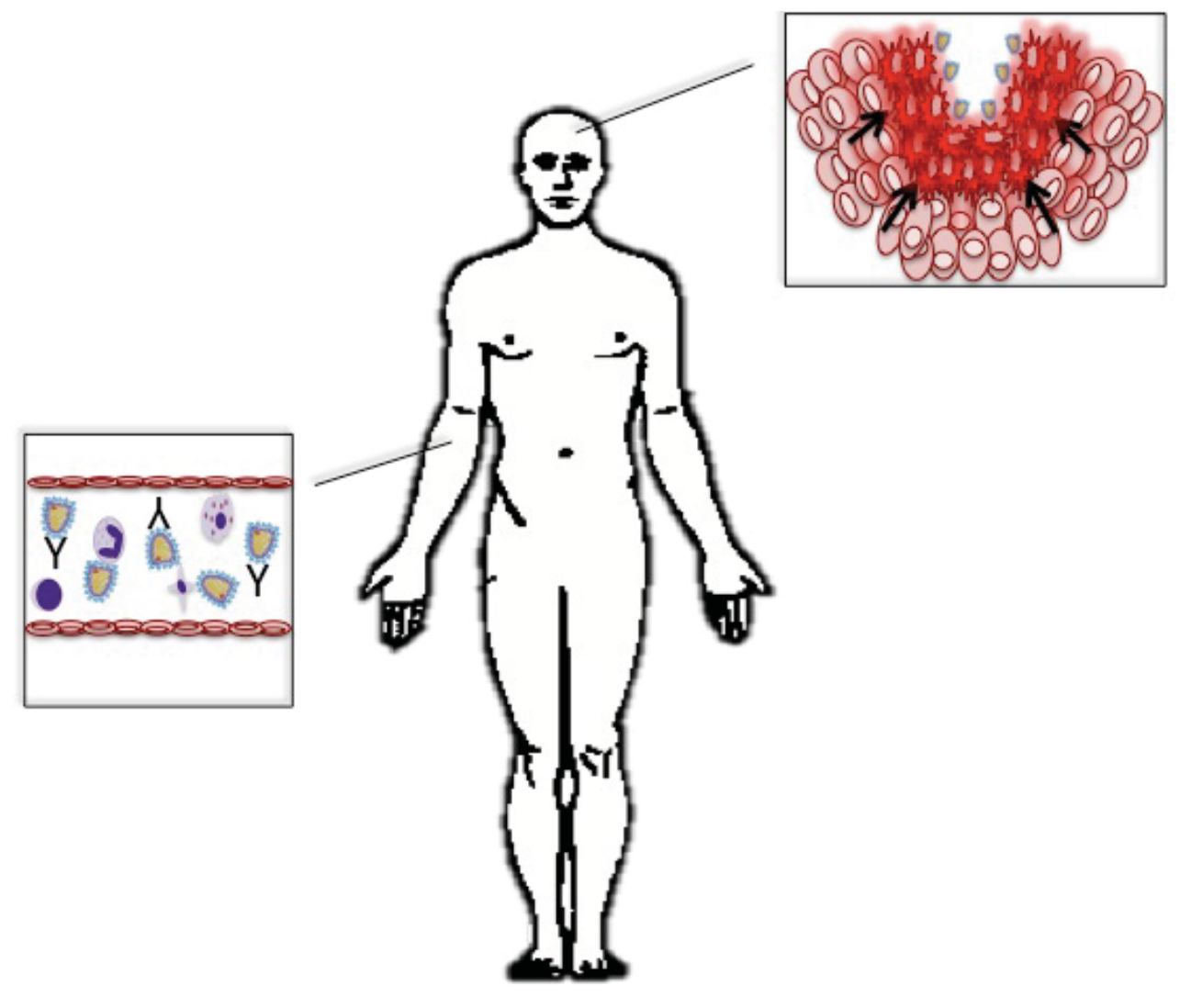

Figure 1-3. Challenges of oncolytic viral therapy

Oncolytic viruses encounter a number of obstacles in achieving therapeutic efficacy. These include uptake and ultimately clearance by immune mediated cells in the systemic circulation as well as neutralization by viral specific antibodies. The tumor microenvironment can also serve as a hindrance to viral oncolysis. For instance, increased pressure secondary to tumor mass and associated inflammation impede the intratumoral infusion of virus. Elements of the extracellular matrix such as collagen may also prevent migration by sequestering virus before it reaches tumor. 
and components of the extracellular matrix (ECM). Collagen, a major component of the ECM, forms protein networks that act as physical barriers to viral penetration often sequestering virions before they can reach less superficial tumor cells. Several OVs have been developed that express genes specific for combating this barrier such as a replication restricted adenovirus expressing collagen cleaving proteins, matrix metalloproteinases (MMPs) [179]. Co-treatment of OV with collagenase has been shown to be effective as well [180].

Just as intravenous administration of chemotherapies result in suboptimal delivery due to the blood brain barrier along with drug metabolism and clearance (reviewed in [181]), OV therapy can be hindered also by the same factors (reviewed in $[182,183]$ ). Intravenous administration exposes virus to circulating cells and factors of systemic host immunity that further inhibit therapy. Though an extremely convenient method as it does not require an invasive procedure for administration, i.v. delivery often causes premature neutralization of virus even before virus is able to reach its target $[184,185]$. These antiviral responses are also associated with morbidity in patients as seen by fever, altered mental status, and other signs and symptoms encountered in a number of OV trials $[65$, 184, 185]. Exposure to the immune system is one of the most vexing challenges faced by viral therapy as it can be both a benefit and impairment to treatment. Unfortunately, preclinical studies have not always been designed in a way to completely understand the involvement of the immune system in OV therapy. Many preclinical studies have utilized immunocompromised animal models to show proof of principle of viral therapy in human-derived tumors $[86,186]$. As we have seen, though antitumor effect is significant in these models, this often does not translate into tumor shrinkage in human trials. Though the immune system can be suppressed in glioma patients, immunity still has an effect on therapeutic efficacy of virus when given intratumorally and systemically. In a preclinical study using an immunocompetent animal model of metastatic lung cancer to evaluate vesicular stomatitis virus (VSV) as cancer therapy, neutralization of i.v. administered virus by circulating antibodies was shown to eliminate delivery of unshielded "naked" virions to tumors $[65,187]$. Several studies have tested a number of methods for circumventing the immune system. One method uses a "cloaking device" which coats virus with chemical conjugates preventing binding of complement and/or uptake by immune cells [188]. Temporarily ablating the immune system is another option and several studies have looked at simultaneous administration of immunosuppressing agents. Cyclophosphamide (CPA) is a multifunctional nitrogen mustard alkylating agent used as an anticancer drug and in the prevention of graft versus host disease. Its immunosuppressive activity has been shown to be caused by the ability to deplete proliferating lymphocytes [189-192]. Rapamycin is another immunosuppressant that works by inhibiting mTORC1, a pro-tumor signaling pathway found to stimulate innate immune responses through type I IFN. It has been shown in a number of preclinical trials to increase viral replication and positive outcomes when given in combination with OV therapies such as VSV, myxoma virus (Myxv), and vaccinia virus (JX-594) [177, 193, 194].

An even more novel approach involves shielding virus from the immune system through the use of cellular vehicles. Many different cell types have been used as cell 
carriers and include $\mathrm{T}$ lymphocytes, mesenchymal progenitor cells, and neural stem cells all showing an affinity for growth within tumor or for the tumor microenvironment. These cells are usually infected, ex vivo, and readministered systemically for viral transport. Upon infection, viruses can be transported by adhering to the surface of carrier cells, as has been shown with retroviral vectors. Adherence to the surface, however, eliminates the ability of the cell carrier to amplify viral dose through active viral replication [195]. Virus can also be transported to sites of tumor by using cells permissive to viral replication. This allows the cell to serve as a viral factory increasing the amount of virus while en route [187, 196-199]. Autologous tumor cells are currently being evaluated for their use as viral carriers. Host derived tumor cells, the actual targets of OVs, instinctively home to sites of pre-existing cancer. They are highly permissive to viral replication and are able to increase original dose of virus by the time they reach the tumor site (Figure 1-4). In a syngeneic model of lung cancer in immunocompetent animals, i.v. administration of $10^{6}$ tumor cells infected with VSV at a multiplicity of infection (MOI) of 10 were able to amplify viral dose by a $10^{5}$ at 24 hours post infection versus $10^{2.5}$ with naked virions [187]. Though this method has sparked some controversy based on existing safety standards and the possibility of seeding tumor in other locations, preclinical results have been promising and in comparison to naked viral therapy in the face of pre-existing immunity, virus-carrying cells were shown to substantially improve survival in animals with established lung tumors, with majority of animals surviving $>100$ days post-treatment $[65,187]$.

Another method for increasing tumor cytotoxicity includes increasing immune responses that are directed against tumor antigens. Gliomas are known to be immunogenic as they possess a number of tumor-associated antigens (TAAs) that can elicit immune responses triggering the influx of various immune cells within and surrounding tumors. Just as we have seen with viruses, tumors often develop ways to evade immune surveillance and responses either by secreting immunosuppressive molecules that dampen immunity (i.e. TGF $\beta$ which has been found to upregulate regulatory T cells) [200-202], by shielding TAAs from patrolling immunity, or by the dysfunction in mechanisms involved in immunological activation [203].

Oncolytic viruses can ignite otherwise weak antitumor immune responses. A phase I trial of the HSV construct, G207, for recurrent GBM demonstrated the ability of this OV to increase glioma-infiltrating immunocytes (GIIs) in treated patients [99, 138]. Viruses can also be used to modify tumor cells making them more detectable to the immune system. By using autologous tumor cells as vehicles, the same method that provides an advantage of shielding virus from adaptive immune responses, virallymodified tumor or viral oncolysate have been shown to also induce an immune response against unmodified tumor cells from which they are derived. Several tumor models have tested this feature using a variety of viruses ranging from influenza, vaccinia, and NDV [204] for treatment of a number of cancers such as melanoma and glioma. A phase II study initiated in 1975 using NDV oncolysate as a post-surgery adjuvant vaccine in patients with AJCC stage III melanoma has shown a $>55 \%$ survival rate at 15 years from vaccine administration. By analyzing the immune repertoire of surviving patients, an increased CD8 $+\mathrm{T}$ cell subset representing terminally differentiated effector cells based 
1. Isolate tumor cells from primary tumor and infect ex vivo with

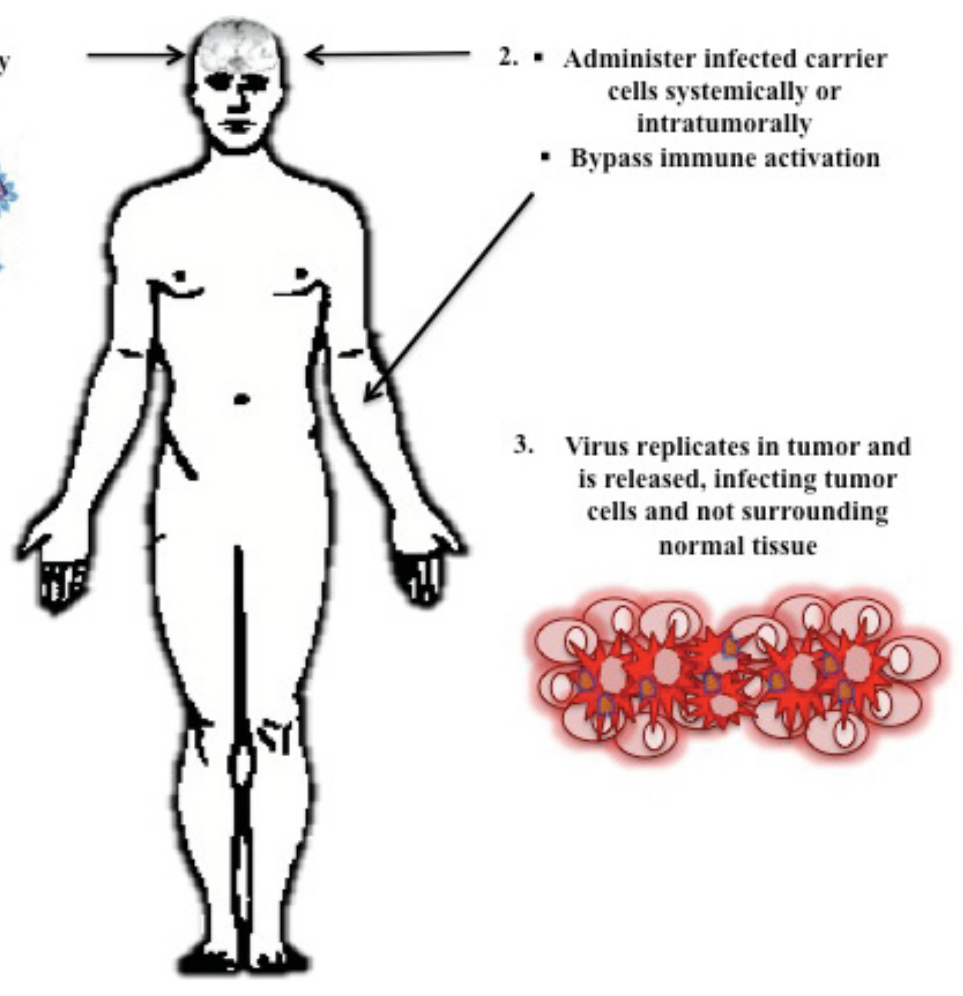

Figure 1-4. Carrier cell strategy

Outlined procedure involved in isolating autologous tumor cells from primary tumor mass and using cells as infected vehicles for the delivery of oncolytic viruses 
on their heightened ability to produce TNF- $\alpha$ and IFN- $\gamma$ were associated with antitumor effect and survival [205]. A more recent Phase I study in 2004 tested the use of irradiated autologous tumor cells as carriers for recombinant Newcastle disease virus (rNDV) as treatment for GBM. Results showed OS of 100 weeks versus 49 weeks in controls with $39 \%$ two year survival versus $11 \%$ in untreated. Increased immune activity directed against tumor was observed in vaccinated patients by an increase in CD8+ tumor infiltrating lymphocytes (TIL) and memory T cell reactivity (demonstrated by IFN- $\gamma$ ELISPOT assay) [206]. As demonstrated, these key inflammatory responses are crucial to long-term survival and anti-tumor activity even after virus has been cleared [45]. More studies will be needed to further evaluate this method of delivery however preliminary results are promising.

\section{Vesicular Stomatitis Virus}

\section{Characterization}

In addition to the previously mentioned viruses that have been or are currently being evaluated in clinical trials for GBM, there are a number of up and coming viral agents that have shown promise in preclinical animal studies as well as in clinical trials for a number of other cancers. One such agent is vesicular stomatitis virus (VSV). VSV belongs to the order, Mononegavirales, an order of characteristically enveloped viruses possessing non-segmented negative sense, single stranded RNA (NNS RNA) genomes. Included in this order are the families Paramyxoviridae, Bornaviridae, Filoviridae, and Rhabdoviridae (Figure 1-5).

A number of important human pathogens belong to these families and include respiratory syncytial virus (RSV) of the Paramyxoviridae family, Ebolavirus of the Filoviridae family, and Borna disease virus of the Bornaviridae family [207, 208]. VSV is a member of the Rhabdoviridae family, the simplest of Mononegavirales viruses. Much of what is known about replication and transcription of these viruses has been determined through studies of Rhabdoviruses particularly, VSV. The two major genera of rhabdoviruses known to infect mammals are vesiculovirus, of which VSV is the prototype, and lyssavirus, of which rabies virus (RABV) serves as the prototype [207]. Lyssavirus genus is made up of not only RABV but also rabies like viruses which are all zoonotic pathogens causing usually fatal encephalitis in animals [209]. Though RABV and VSV share very similar structural and genetic makeup as members of the same viral family, disease sequelae from their respective natural infections are very different, with RABV causing a slow and almost uniformly fatal and destructive disease and VSV causing an acute and generally mild disease with rare neurological symptoms [210].

Rhabdoviruses infect a wide range of hosts from vertebrates and invertebrates to plants. Aside from a few species including rabies that primarily infect vertebrates, most other rhabdoviruses, including VSV, are considered arboviruses, requiring transmission via arthropod hosts such as blackflies, sandflies, and mosquitoes. There are several VSV 


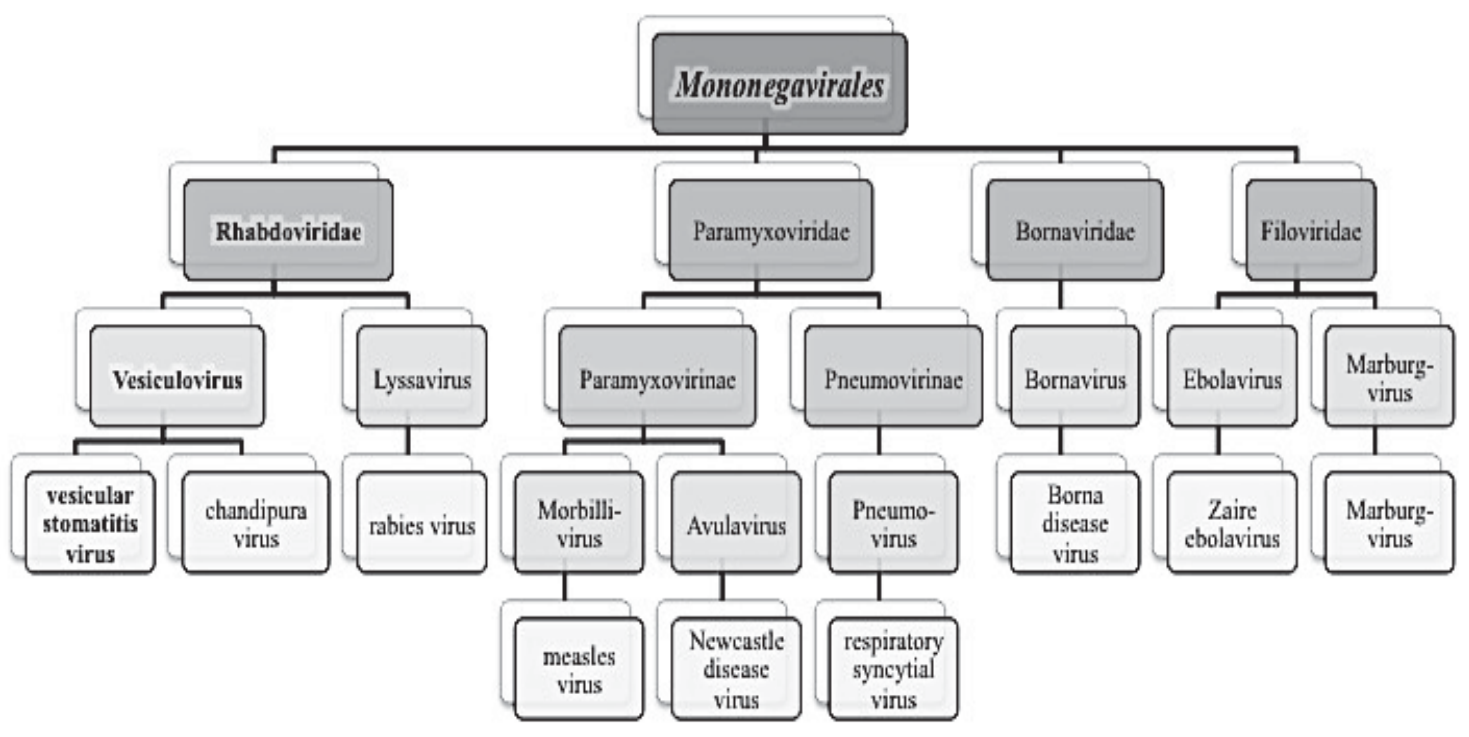

Figure 1-5. Select representatives of the order, Mononegavirales

Source: Ictvonline.org- International Committee on Taxonomy for Viruses 
serotypes found worldwide; however, two distinct serotypes are predominantly found within the Americas, which include New Jersey (VSV-NJ) and Indiana (VSV-IND). The most prevalent serotype is VSV-NJ, accounting for $\sim 80 \%$ of natural infections [211, 212]. These serotypes share approximately $50 \%$ amino acid sequence homology and infection of one serotype does not confer protection against future infection of the other $[207,213]$.

\section{Disease Manifestations and Modes of Transmission}

Vesicular stomatitis (VS) is the disease caused by VSV infection and presents as an acute, nonfatal, but debilitating disease in cattle, swine, and horses. Signs of infection in animals include formation of fluid-filled vesicular lesions of the mouth, tongue, hooves, and teats [214], which often coalesce and rupture leaving areas of painful ulcerations that may cause infected animals to refuse food and water. Eventually, animals experience severe weight loss with lowered milk production and productivity [215]. Manifestations of VS are often confused with foot and mouth disease, another infection of livestock caused by the picornavirus, foot and mouth virus. This disease is known to be more debilitating therefore establishing the correct diagnosis is paramount as it can have unfavorable agricultural and economical consequences if spread of infection is not controlled. Geographically, VS is more prevalent in areas surrounding the borders of Central and South America with outbreaks occurring seasonally, during warmer temperatures [212]. In the United States, outbreaks typically occur every 10 years however, within the last decade, they have been more consistent with bursts of infection observed almost annually since 2005 [216].

As noted, VSV is an arbovirus, therefore, natural infection of vertebrate hosts follows the bite of an infected insect. Additional modes of transmission may occur and include aerosolization or contact with skin lesions of the infected. Experimental inoculation of livestock can cause clinically relevant disease that is dose dependent and varies based on route of administration. Viremia in experimentally inoculated livestock is rare but possible [217]. On the other hand, experimental inoculation of mice results in viremia and often death due to encephalitis depending on age of animal (adult versus nestling) and route of administration [212, 218-220].

VSV is not considered a major human pathogen, unlike its close relative, rabv, or other vesiculoviruses found worldwide such as Chandipura virus and Isfahan virus [221, 222]. Resulting from its broad tropism, the virus can infect humans, however, infection is usually asymptomatic or may manifest as mild flu-like symptoms. Seroconversion is rarely detected in the general population and those with positive serology usually have either a history of exposure to infected cattle or have handled virus in a lab setting [207]. 


\section{VSV Structure}

Due to its simplicity, VSV has been the most extensively studied virus in its order and these studies have provided much of what is presently known about the life cycle of Mononegavirales as a whole. Other attributes of VSV that deem it the prototype of Rhabdoviruses aside from its simple genome include its broad cell tropism and high yields in cell culture [207]. The VSV genome consists of 11.2 kilobases $(\mathrm{kb})$, which serially transcribes 5 monocistronic mRNAs used to translate the 5 major viral proteins: the nucleoprotein $(\mathrm{N})$, the phosphoprotein $(\mathrm{P})$, the matrix protein $(\mathrm{M})$, the glycoprotein $(\mathrm{G})$, and the large RNA polymerase (L) (Figure 1-6).

As most Rhabdoviruses, excluding some bacillus shaped plant Rhabdoviruses, mature VSV virions have a characteristic bullet shape measuring 180nm x $75 \mathrm{~nm}$. These virions acquire an envelope composed of the host cell membrane from which they bud. Embedded in and protruding from the envelope are $\mathrm{G}$ proteins arranged in trimeric spikes. Surrounded by the envelope is a core ribonucleoprotein (RNP) composed of the NNS RNA genome encapsidated by $\mathrm{N}$ proteins keeping the genome compact hence resistant to cellular RNase. Molecules of viral $\mathrm{M}$ protein are attached to the inside leaflet of the viral envelope between the membrane and the nucleocapsid core keeping the RNP in a rigid helical formation [207]. The RNA-dependent RNA polymerase (RdRp) is a complex formed by the $\mathrm{P}$ and $\mathrm{L}$ proteins (L-P3 complex) during transcription and $\mathrm{L}, \mathrm{N}$, and $\mathrm{P}$ (L-N-P complex) proteins during replication. The active RdRp is also responsible for additional processing of transcripts which include the addition and methylation of a $5^{\prime}$-cap and addition of a 3'-poly(A) tail (reviewed in [207, 223].

\section{VSV Replication Cycle}

As with most RNA viruses, the replication cycle of VSV occurs exclusively in the cytoplasm. Each VSV structural protein plays an important role in these events beginning with adsorption of virus at the cell membrane, followed by entry and uncoating, transcription, replication, assembly, and finally culminating with budding of infectious particles from the host cell.

Specifically, binding of virions to the host cell surface occurs between viral glycoprotein and a universally expressed host cell receptor [207]. Phosphatidylserine (PS) was previously considered as the possible cellular receptor, however newer data challenge this proposal with one study suggesting the role of the endoplasmic reticulum chaperone protein gp96 as a facilitator of viral attachment with host cells [224-227]. Following adsorption, virus is endocytosed through a clathrin-dependent receptormediated pathway. As virus is exposed to decreasing $\mathrm{pH}$ during endocytosis, $\mathrm{G}$ protein undergoes a conformational change resulting in fusion of the viral envelope and endosomal membranes. As virus traffics through endosomal compartments, $\mathrm{M}$ releases its rigid hold on the RNP while remaining bound to the endosomal membrane allowing the RNP core to then be released into the cytoplasm [228]. This uncoating step leaves the RNP available for transcription by the pre-packaged RdRp in order for synthesis of new 


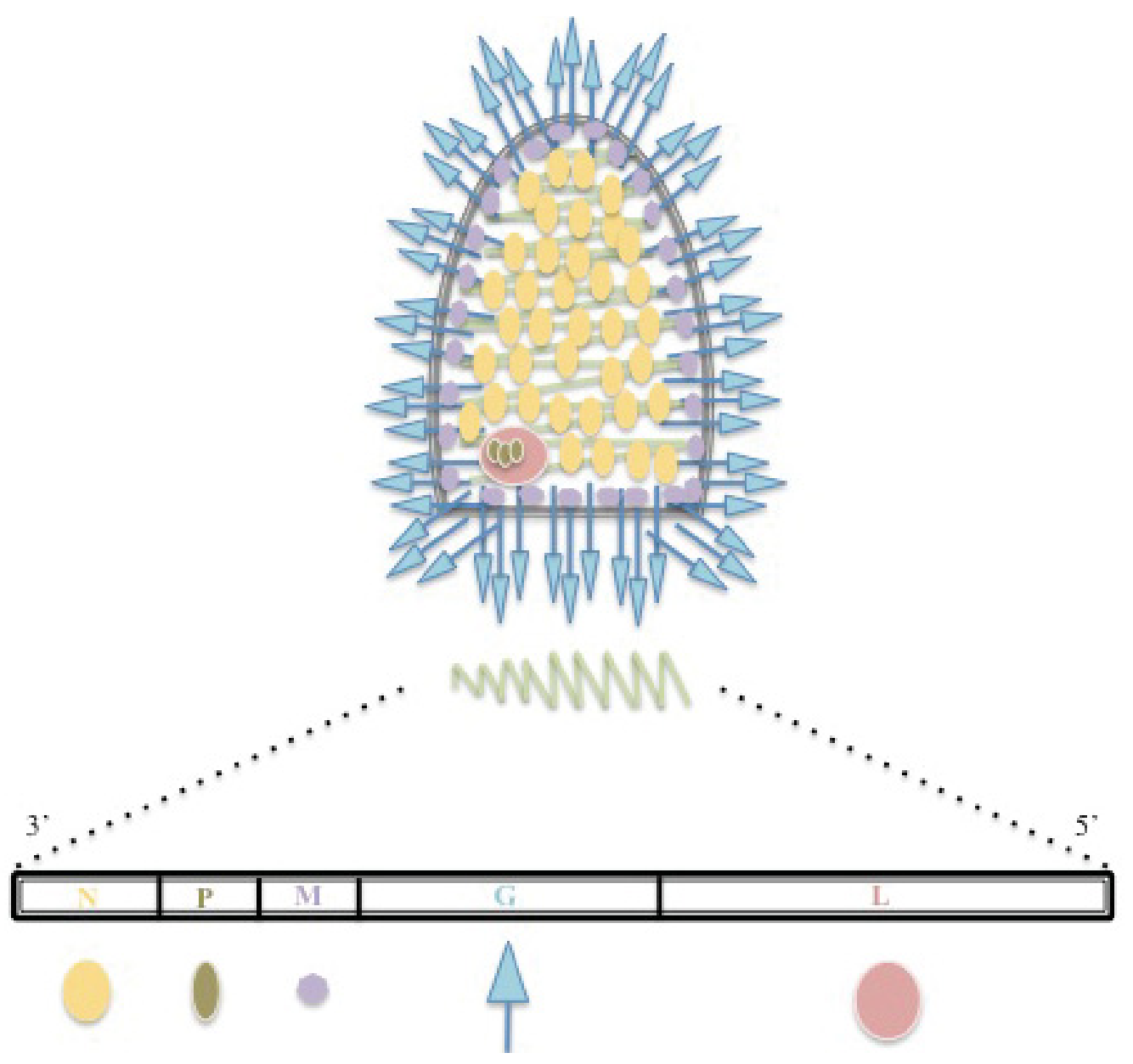

Figure 1-6. Vesicular stomatitis virus

Schematic of the characteristic bullet-shaped vesicular stomatitis virion, depicting the layout of the 11.2 kilobase negative single stranded RNA viral genome from 3' to 5'. $\mathrm{N}$, nucleocapsid; P, phosphoprotein; M, matrix; G, glycoprotein; L, large RNA polymerase 
viral proteins needed for subsequent rounds of transcription and ultimately replication. Directionality of transcription of the negative sense RNA genome by the active polymerase occurs uninterrupted from 3' to 5 ' in the specific order of 3'( $\ell$ )-N-P-M-G-L(t) 5 ' with the first event being synthesis of the 47 nucleotide RNA leader sequence, $\ell$, located in the 3' untranslated region (UTR)of the genome. This sequence is responsible for encouraging transcription of viral genes. The last event involves transcription of the trailer sequence, $t$, located in the 5' UTR. As mentioned, each mRNA contains a 5' cap and terminate with a poly(A) tail. Transcriptional attenuation occurs with an approximately $20 \%$ to $30 \%$ decrease of transcript synthesis at each contiguous gene junction. This results in a gradient of mRNA from the more abundant 3' located $\mathrm{N}$ transcript to the least abundant 5' located L transcript (reviewed in [207].

In addition to its role in transcription, $\mathrm{RdRp}$ is responsible for making new genome that can be used for amplifying transcription and for packaging into new virions. Therefore, a transitional product involving a positive-sense, antigenome replication intermediate (RI) is made by the RdRp for this purpose. Once message is made and newly synthesized $\mathrm{N}$ and $\mathrm{P}$ accumulate, then genome replication can occur [207]. Next assembly of new virions ensues as newly synthesized genomes are encapsidated and then condensed into a skeleton form. The recent 3D constructed image by cryo-electron microscopy of the VSV virion has revealed that assembly is initiated at the tip of the bullet shaped virion with $M$ serving as a facilitator through its multiple interactions with neighboring M proteins, overlying plasma membrane, and the underlying RNP [229].

The ultimate step in the life cycle is budding of newly made, mature virions preferentially from the basolateral surface of infected epithelial cells [230, 231]. These areas have been found to harbor $\mathrm{G}$ protein containing lipid domains [232] along with higher concentrations of membrane bound M protein [233] and cellular budding machinery $[231,234]$. As these new virions are released from the cell, they attain a new envelope derived from the host cell membrane [207].

\section{The Multifunctional Matrix Protein}

In order to promote quick and efficient replication, a number of viruses encode for proteins that have multiple functions in the viral life cycle $[46,235,236]$. One such example for VSV is the matrix protein (Figure 1-7). M is comprised of only 229 amino acids making it the smallest of virally encoded proteins weighing in at $26 \mathrm{kDa}$. Though it is the smallest, it is also the most abundant protein in the VS virion and participates in many aspects of the viral life cycle from assembly to budding [207]. In addition to the full-length matrix protein, M1, VSV expresses two additional shorter matrix polypeptides known as M2 and M3 (Figure 1-7C) [237]. These shorter proteins are synthesized from the same open reading frame, but from downstream AUG codons therefore exhibiting Nterminal truncations compared to full length M1. M2 starts with methionine at position 33 and M3 at Methionine 51. These proteins are incorporated into virions at very low levels and, as will be discussed, have been found to play a role in the virus's characteristic cytopathic effects during infection [237]. 
A.

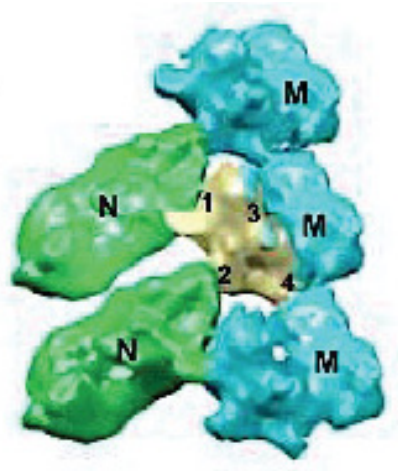

B.

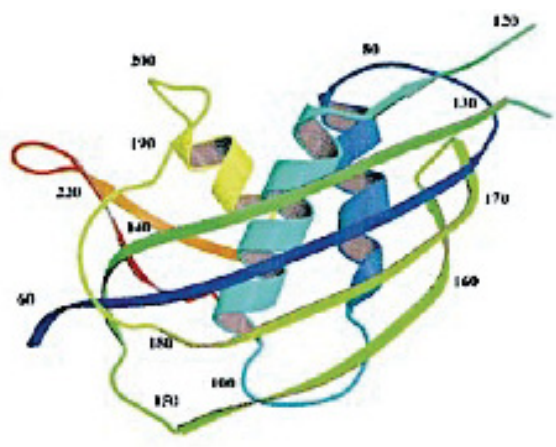

C.

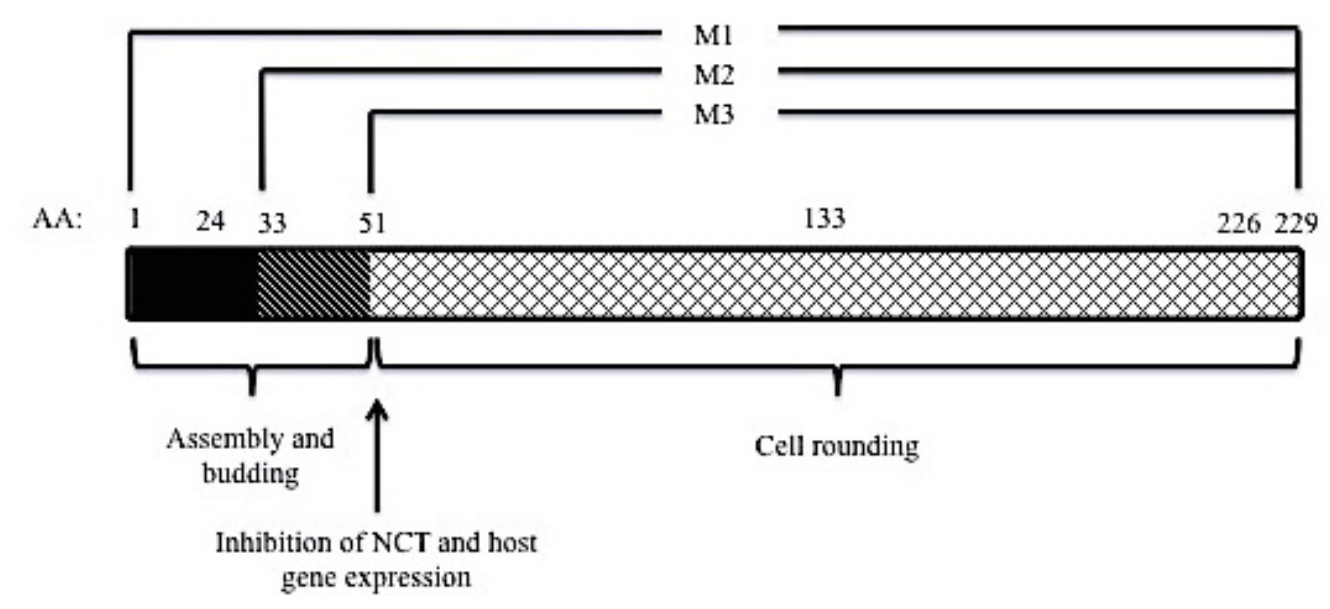

Figure 1-7. The matrix (M) protein

(A) Reconstruction of cryo-EM images illustrating the four interactions of the $\mathrm{N}$-terminus of matrix with adjacent matrix proteins and with adjacent nucleocapsid proteins. (B) Crystal structure of the thermolysin cleaved globular core of the matrix protein revealing 3 alpha helices surrounded by 7 beta sheets. (C) Schematic of the full-length matrix protein depicting important functional domains including the amino acid start sites for matrix variants, M2 and M3. (A) reprinted with permission from Peng G, J. Tsao, S. Schein, T. Green, M. Luo, and Z. Hong Zhou (2010.) CryoEM model of the bulletshaped vesicular stomatitis virus. Science 327 (5966): 689-93 [229] and (B) Gaudier, M., Y. Gaudin, and M. Knossow (2002). Crystal structure of vesicular stomatitis virus matrix protein. Embo J 21:2886-92 [238]. Cryo-EM, Cryo-electron microscopy; AA, amino acid; NCT, nucleocytoplasmic transport 


\section{Matrix Structure}

The crystal structure of the M protein was solved in 2002 by Gaudier et al and was shown to be similar in structure to proteins of other NNS RNA viruses important in budding and viral assembly [238-241] (Figure 1-7B). In order to achieve crystallization, matrix was treated with thermolysin, cleaving the protein at the $\mathrm{N}$ terminus and at the hydrophobic loop known to be involved in M polymerization. Resultant thermolysin cleaved $\mathrm{M}$ monomers $\left(\mathrm{M}_{\mathrm{th}}\right)$ corresponded to amino acids 48-121 and 122-229 and these two fragments were held together by a hydrophobic, globular core [238]. A number of domains have been found to be responsible for the multi-functionality of matrix and a more recent 3D structure created from cryo-electron microscopic studies has shed more light on the topography of these domains and how they interact with other proteins in the virion. It was shown that the $\mathrm{N}$ and $\mathrm{M}$ layers in the virion are both made up of a separate single helix and the $\mathrm{N}$ terminus of $\mathrm{M}$ (M-hub) has 4 contact points, important in maintaining the virion's rigid bullet structure (Figure 1-7A). Contact point 1 joins Mhub to an $\mathrm{N}$ in the upper helical turn, while $\mathrm{M}$-hub contact-point 2 connects $\mathrm{M}$-hub to an $\mathrm{N}$ subunit in the lower helical turn, confirming previous data that the N-terminus (AA421) of $\mathrm{M}$ is important in binding to the RNP core [242]. Contact-point 3 binds laterally to the $\mathrm{C}$-terminus of $\mathrm{M}\left(\mathrm{M}_{\mathrm{CTD}}\right)$ of the trailing $\mathrm{M}$ subunit in the same helical turn while contact-point 4 binds to $\mathrm{M}_{\mathrm{CTD}}$ of the $\mathrm{M}$ in an upper helical turn [229]. This further validates what is known about the protease sensitive loop of matrix[243, 244].

The functional domains of matrix have been shown to be genetically separable from each other with domains involved in assembly and budding being different from those involved in CPE [242, 245] (Figure 1-7C). In addition, these functions are carried out in very different parts of the infected cell reflected by the approximately $10 \%$ of matrix associated with the inner leaflet of the plasma membrane, where assembly and budding is known to occur, with the remaining matrix located either in the cytoplasm or, to a lesser degree, in the host cell nucleus. With so many roles being carried out in different cellular compartments by one agent, it is not surprising that separate matrix populations created by genetic truncations could provide the virus with several options for carrying out its functions simultaneously.

\section{Assembly and Budding}

During VSV assembly, as the RNP begins to form a helical structure, M subunits attach to the outer surface of the nucleocapsid and rigidify the forming RNP skeleton starting from the tip to the trunk or base of the forming bullet shaped structure. The exposed surface of M not bound by neighboring $\mathrm{M}$ or the RNP creates an area for binding $\mathrm{G}$ trimers associated with the soon to be acquired envelope membrane [229]. The importance of $\mathrm{M}$ to assembly has been demonstrated by deletion or mutation of $\mathrm{M}$ especially seen with temperature-sensitive mutants of VSV that produce very low levels of spherical or pleomorphic-shaped particles at non-permissive temperatures.

Complementation of these mutant viruses with wildtype matrix restores the classical bullet shape of the virions [246]. 
The multiple interactions of M with other VSV structural proteins also help promote viral budding from infected host cells [229, 231]. Significance of M in budding was demonstrated in studies showing the transient expression of $\mathrm{M}$ alone causes evagination of host cell membrane and ultimately release of membrane-enclosed vesicles [247]. Point mutations in the proline rich motif, ${ }_{24} \mathrm{PPxY}_{27}$, located in the $\mathrm{N}$ terminus of $\mathrm{M}$ (late-budding or L-domain) greatly diminishes vesicular budding and the infection with viruses possessing mutations in the L domain show a decrease in budding by $70 \%$ of wildtype infection. This motif has been shown to facilitate budding through interactions with host cell E3 ubiquitin ligase, Nedd4, via its WW-domains [241, 248].

\section{Cytopathic Effects}

In addition to its role in assembly and budding, $\mathrm{M}$ is responsible for the phenotypic cytopathic effects (CPE) observed during VSV infection. CPE manifests as cell rounding, activation of apoptosis, and inhibition of host gene expression. In baby hamster kidney cells (BHK), a major cell model used in studies of VSV, CPE can be observed as early as 1 to 2 hours post-infection at high multiplicities of infection (MOIs) or 4-5 hours post-infection at lower MOIs [241]. M's importance in CPE stemmed from a number of studies including those using temperature sensitive mutant viruses. For instance, tsO82, a mutant found to have a mutation in matrix, was non-cytopathic in chick embryo fibroblasts [249] and this defect correlated with an inability to shutoff host gene expression [245]. The fact that expression of $\mathrm{M}$ alone, in the absence of other viral proteins, is able to elicit cell rounding and apoptosis emphasizes its role in CPE [242, 250-254].

\section{Cell rounding and apoptosis}

Cell rounding and induction of apoptosis are well-known characteristics of VSV infection. This change in cell morphology is an effect of cytoskeletal dysfunction specifically due to matrix interaction with tubulin as co-immunoprecipitation revealed interaction between the acidic C-terminus of tubulin and the basic $\mathrm{N}$-terminus of $\mathrm{M}$ [251]. More recent studies, however, revealed that matrix may not associate with actin or tubulin for at least 1 hour post-infection [228]; however, interaction between $\mathrm{M}$ and dynamin [255] through a domain located in the N-terminus of matrix may indirectly affect actin components of the cytoskeletal network [256]. In support of M's role in cell rounding, a temperature sensitive mutant, tsG33, known to harbor mutations in the matrix protein, was shown to be deficient in cell rounding in BHK-21 cells at non-permissive temperatures. In this same study, $\mathrm{M}$ was able to cause cell rounding in the absence of other viral proteins and blocking $\mathrm{M}$ activity by microinjection of complimentary antisense oligonucleotides inhibited cell rounding [250].

Induction of apoptosis is also a feature of VSV-induced CPE. Apoptosis was first verified by the presence of DNA laddering and nuclear fragmentation that occurred simultaneously with, but was not dependent on, viral replication $[257,258]$. The 
expression of $\mathrm{M}$, alone, is able to elicit apoptosis and is evident by activation of caspase3 in HeLa and BHK cells [259]. M's ability to activate apoptosis leads to cell rounding and in cells where $\mathrm{M}$-induced apoptotic pathways are blocked, cell rounding is also inhibited [252].

\section{Inhibition of host gene expression}

Another feature of viral-induced CPE includes the inhibition of host gene expression or host cell shutoff. There are several stages of gene expression that can be inhibited and these not only include transcription and translation of the host genome but also transport of message from the nucleus into the cytoplasm as well (e.g. nucleocytoplasmic transport or NCT) (Figure 1-8). As previously mentioned, leader RNA ( $\ell$ ), located in the 3' untranslated region plays a significant role in encouraging viral transcription [207]. Along with this role, $\ell$ has been shown to be involved in inhibition of host cell gene expression as it migrates to the nucleus early in infection and inhibits initiation of RNA polymerase II and III dependent transcription [260-262]. However, $\ell$ is not sufficient to shutoff host gene expression on its own and another viral protein was identified as playing a major role in this process. That protein was found to be M [263, 264]. Not only is matrix implicated as the viral protein involved in host shutoff, Lyles et al showed that its ability to do this leads to induction of apoptosis and cell rounding [253, 254].

\section{Inhibition of transcription and translation}

Evidence of M's role in host shutoff include its ability to localize to the nucleus of infected cells [265] along with studies showing the temperature sensitive matrix mutant, tsO82, expresses defects in host shutoff during infection [245]. Expression of M, alone, inhibits not only host directed transcription but also its own transcription when expressed from plasmids transcribed by host RNA polymerase II, a property not possessed by other viral proteins $[242,254]$. This inhibition was shown to be independent of promoter type (hIFN $\beta$ cellular promoter versus SV40 viral promoter) [266]. Activity of RNA polyermase (RNAP) I, II, and III are effected with RNAP II being the most sensitive to viral inhibition $[267,268]$ due to inactivation of transcription factor, TFIID [269, 270].

VSV host shutoff also involves inhibition during translation through alterations of eIF4F initiation complex $[271,272]$. Inhibition at this level favors mRNAs already existing in host cells prior to infection while new mRNAs or mRNAs present after infection are not inhibited. This along with the fact that VSV inhibits host transcription helps promote translation of viral mRNA as increases in viral translation occurs simultaneously with decreases in host mRNA translation [273]. 


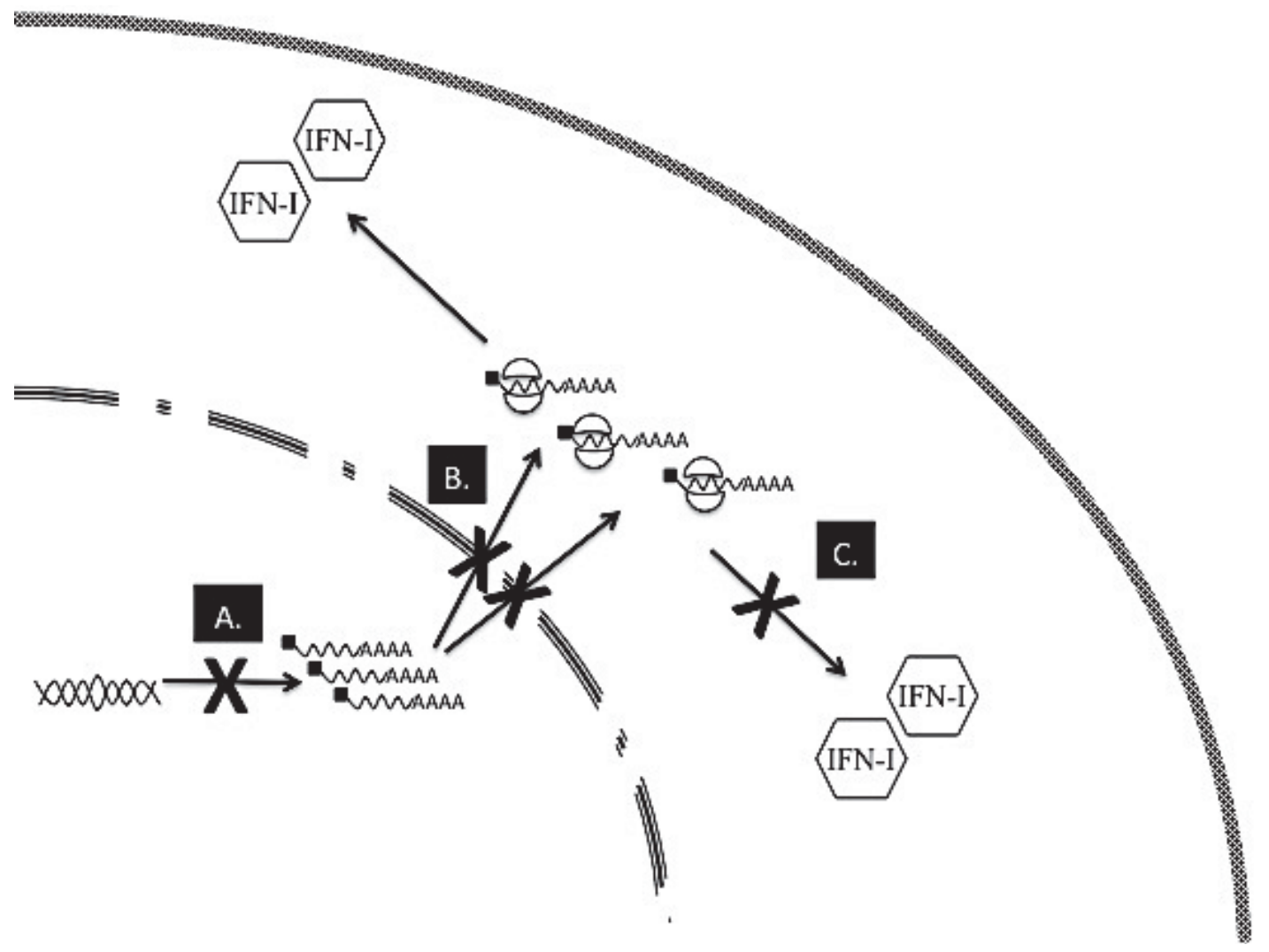

Figure 1-8. Multiple steps in the inhibition of host gene expression by VSV matrix protein

There are multiple steps in the process of host gene expression that may be targeted by VSV in order to promote the viral life cycle. (A) Inhibition of transcription particularly of genes transcribed by host RNA polymerase II (B) inhibition of NCT by binding at the nuclear pore complex and (C) inhibition of translation caused by viral induced alterations of the eIF4F. 


\section{Inhibition of nucleocytoplasmic transport (NCT)}

Lastly, inhibition of NCT is a major feature of VSV-induced CPE specifically in inhibition of host gene expression. Inhibiting movement of mRNA into the cytoplasm would theoretically prevent expression by decreasing the availability of mRNA to the translation apparatus. As stated, there is a population of matrix found in the nucleus unlike other viral proteins [265] and movement of M into the nucleus is supported by the presence of nuclear localization signals (NLS) [274]. NCT inhibition occurs when matrix is bound to the nuclear envelope and this activity is reversed in the presence of a mAb directed against $\mathrm{M}(\alpha \mathrm{M})$ [275]. At the nuclear envelope, $\mathrm{M}$ specifically binds to the nuclear pore complex (NPC) where it alters NCT activity mediated by the nucleoporin, Nup98, and shuttling mRNA export factor, Rae1. Mutant viruses unable to bind at the NPC are able to migrate to the nucleus but are defective in inhibition of transcription andNCT seen by an increase in the amount of nuclear mRNA [276-278].

More recent studies question the actual role of Matrix and Nup98/Rae1 interactions in host cell shutoff during VSV infection. Rae1 knockout cells were shown to have no effect on the accumulation of nuclear mRNA after wildtype infection however there was a decrease in the inhibition of host transcription. In addition, without Rae 1, M is unable to bind to Nup98 although $\mathrm{M}$ is able to bind Rae1 in the absence of Nup98. These data implicate the importance of Rae1 as a bridge for interaction between matrix and other host factors such as Nup98 and that these connections are important in CPE observed during VSV infection. This also suggests a more vital role for these complexes in the inhibition of transcription [279].

\section{Matrix domains important for CPE}

In addition to the aforementioned domains important to viral assembly and budding, genetically separable domains have also been identified as being important in viral induced CPE [242, 252]. These domains are summarized in Table 1-4. PSAP domain located between amino acids $37-40\left({ }_{37} \mathrm{PSAP}_{40}\right)$ at the N-terminus of matrix was initially studied for its possible role in viral budding mechanisms. It was believed to act as an L domain considering this motif is highly conserved in other vesiculoviruses [222, $280]$ and has been proven to function as an L domain in a number of other viruses including hepatitis E virus and Ebola virus [281,282]. In addition, another highly conserved region in the matrix protein much like PSAP, the ${ }_{24} \mathrm{PPxY}_{27}$ motif, has been confirmed as an L-domain whose function is vital to VSV budding [241]. Studies eventually showed that this is not the case and that mutations in ${ }_{37} \mathrm{PSAP}_{40}$ alone have no effect on budding; however, in combination with PY mutations, ${ }_{37} \mathrm{PSAP}_{40}$ may help rescue the defective budding phenotype of PY mutants [283, 284]. Recent studies have implicated PSAP as being more vital to viral CPE especially as it relates to cell rounding, caspase activation, and ultimately, viral yield. Substitution of 4 alanines for PSAP (PS $>$ A4) resulted in a highly attenuated virus in vitro and in an in vivo mouse model. On the other hand, $\mathrm{PS}>\mathrm{A} 4$ resulted in the opposite phenotype in arthropod cell lines, with enhanced CPE seen by increased cell rounding, detachment, and activation of caspase 3 
Table 1-4. Functional domains of VSV matrix protein

\begin{tabular}{|c|c|c|c|}
\hline Role of Matrix & $\begin{array}{c}\text { Amino Acid } \\
\text { Domain Location }\end{array}$ & Specific Motif & References \\
\hline Dynamin Binding & $1-10$ & $N S$ & {$[255]$} \\
\hline RNP association & $1-24$ & $N S$ & [229] \\
\hline \multicolumn{4}{|l|}{ Budding } \\
\hline $\begin{array}{l}\text { 1. Binds to WW } \\
\text { motifs on cellular } \\
\text { proteins (Nedd4) }\end{array}$ & $24-27$ & PPPY & $\begin{array}{c}{[229,231,241,246-} \\
248]\end{array}$ \\
\hline 2. Binds tSG101 & $37-40$ & PSAP & {$[282,283]$} \\
\hline $\begin{array}{l}\text { Mitochondrial } \\
\text { targeting }\end{array}$ & $33-67$ & $N S$ & {$[285]$} \\
\hline \multicolumn{4}{|l|}{$\mathrm{CPE}$} \\
\hline $\begin{array}{l}\text { 1. Cell rounding } \\
\text { and apoptosis }\end{array}$ & $\begin{array}{l}\text { a. } 33-51 \\
\text { b. } 37-40\end{array}$ & $\begin{array}{l}\text { a. } N S \\
\text { b. PSAP }\end{array}$ & $\begin{array}{ll}\text { a. } & {[237,242,252,} \\
& 259,265] \\
\text { b. } & {[284,286,287]}\end{array}$ \\
\hline $\begin{array}{l}\text { 2. Inhibition of host } \\
\text { gene expression }\end{array}$ & $51-59$ & $N S$ & $\begin{array}{c}{[245,275,277-279} \\
288]\end{array}$ \\
\hline Assembly & $123-125$ & LXD & {$[231,238,243,244]$} \\
\hline Self association & $120-129$ & PAVLA & {$[229,238,243,244]$} \\
\hline
\end{tabular}

Note: NS, not specified 
$[286,287]$.

Another domain important to viral CPE was addressed in the previously mentioned studies using temperature sensitive mutant, tsO82 [249]. This mutant expresses a mutation in matrix in which a methionine at position 51 is replaced with an arginine (M51R). This substitution renders it defective in host gene expression inhibition, and through complementation with assembly mutants, a genetic distinction between these functional domains was further confirmed [245]. Studies using M51R expressing plasmids have shown that while wildtype matrix co-localizes with components of the nuclear pore complex at the nuclear rim, M51R mutants do not suggesting NPC-matrix interactions are determined by this domain and are important for the inhibition of host gene expression [277, 288]. In addition, another genetically modified matrix mutant, $\mathrm{M}(\mathrm{D})$, which possesses alanine substitutions at positions 52-54 show that these mutations do not inhibit the ability of matrix to migrate into the nucleus, however by their inability to interact with the NPC, inhibitory activity on gene expression is diminished [275].

Jayakar and Whitt eventually demonstrated a major influence of M51 mutations in the expression of the matrix protein. Mutations introducing an alanine at this position in combination with the same mutation at position 33 (M33, 51A) prevented the synthesis of the shorter matrix protein products, M3 and M2, respectively, and resulted in synthesis of only the full-length matrix protein, M1. These proteins were shown to be important in eliciting viral induced $\mathrm{CPE}$ and virions that do not express these proteins have a delay in cell rounding that can be recovered by co-expression of M2 and M3. Surprisingly in these mutants, virus yield is not affected and inhibition of host gene expression determined by protein visualization of $\left[{ }^{35} \mathrm{~S}\right]$ Met labeled infected cell extracts showed no significant differences from wild-type infection [237].

In all, viruses harboring mutations corresponding to position 51 are attenuated likely due to their inability to inhibit the expression of specific genes important in antiviral responses such as Type I interferons (IFN $\alpha / \beta)$ [266, 289-291]. It is the M51 mutation specifically that has been the main focus of studies geared toward developing VSV as an oncolytic virus. Furthermore, this mutation is thought to be responsible for the virus's preferential replication in tumor cells lacking a functional IFN response pathway [292-294].

\section{VSV as an Oncolytic Agent}

\section{Proposed Mechanism of Oncolysis}

As described, the matrix protein's role in VSV cytopathic effects are illustrated through its ability to prevent the expression of host genes, including those crucial to regulating viral infection. Previous studies have not only demonstrated inhibition of IFN expression by wildtype matrix, but also that viruses expressing a host shutoff defective 
matrix protein are actually inducers of IFN [292]. Many tumors harbor defects in the interferon pathway giving them a survival advantage over normal cells while leaving them susceptible to infection by numerous viruses including VSV. To this end, it should be expected that VSV would have a growth advantage in transformed cells based on their inability to fight off infection. This expectation has been proven true in a number of tumor cells from different origins including prostate, breast, and colon. Furthermore, pretreating cells with IFN is able to protect normal cells from infection while tumor cells, with very little ability to respond to IFN, remain sensitive to viral infection [291].

By using matrix mutant, with the inability to shut off host gene and protein expression, the oncolytic potential of VSV vectors is improved. These mutants create a similar environment much like the one observed with IFN pretreatment. During infection, accumulating IFN forms a "cytokine cloud" around surrounding cells that stimulates downstream signals protecting IFN responsive cells from further infection while the IFN-resistant tumor cells are left vulnerable (Figure 1-9) (reviewed in [289]).

\section{IFN Pathway}

In understanding the mechanism of VSV oncolysis, it is important to have a general understanding of normal antiviral responses. Innate immune responses are crucial in the survival of cells against VSV infection as the virus is extremely sensitive to the antiviral actions of the IFN pathway. In animals with defective innate responses, such as IFN receptor (IFNAR)- deficient mice, VSV rapidly replicates to high levels before the adaptive immune response can neutralize virus [295]. Interferons (IFNs) are specific to vertebrates [296] and virtually all nucleated cells maintain the capacity to produce and respond to IFN [297].

There are two main families of IFN, designated type I $(\alpha / \beta)$ and type II $(\gamma)$. The 26 genes coding for Type I IFNs are found on human chromosome 9 and include 13 IFN $\alpha$ genes, 11 of which are functional, a single IFN $\beta$, and 11 IFN pseudogenes (reviewed in [297]). More recently added type I IFNs include IFN $-\delta,-\varepsilon,-\kappa,-\tau$, and $-\omega$ (reviewed in [298]). Type I IFNs have multiple antiviral effects including the ability to activate immune effector cells such as natural killer (NK) cells, macrophages and dendritic cells (DCs) $[296,299]$. Though IFNs are considered major players in innate immunity, their ability to influence DC maturation indicates a role in bridging the innate and adaptive immune systems. IFNs also have antitumor effects, demonstrated by their connection to the tumor suppressor, p53 pathway, which is dysregulated in cancers of all types [300]. The IFN-I receptor, IFNAR, is found in the plasma membrane and is composed of two major subunits, IFNAR1 and IFNAR2. Separately, each subunit serves as a docking site for intracellular signaling molecules. Once bound to IFN, these subunits become a heterodimer triggering an elaborate downstream antiviral signaling cascade that will be discussed in more detail (Figure 1-9B). All Type I IFNs bind to this receptor, however, depending on the bound IFN, distinct downstream signaling can be observed [301]. 
Figure 1-9. VSV and the IFN pathway

(A) PRRs such as RIG-I and TLRs recognize pathogens, setting off an alarm in the form of a downstream signaling cascade that leads to the translocation of IRF3/7 and NFKB to the nucleus where they initiate the expression of IFN-I genes. (B) IFN protein is translated and secreted by cells into the extracellular environment where it binds to its receptor. IFN works in an autocrine and paracrine fashion to increase the translocation of Stat 1 and 2 to the nucleus where they upregulate a host of IFN stimulated genes (ISGs). (C) During wtVSV infection, the matrix protein is able to block multiple stages of host gene expression including genes involved in creating antiviral responses. This enables viral replication and subsequent infection of surrounding cells. (D) During infection with VSV matrix mutants, $\mathrm{M}$ is unable to block host gene expression, therefore, allowing IFN gene expression to continue. Tumor cells, with defects in host gene expression are unable to create an IFN induced antiviral state, which allows uncontrolled spread of virus. (E) Cells having an intact IFN response, are able to amplify IFN signal by downstream ISGs, protecting other IFN responsive cells from viral infection at multiple stages of the viral life cycle. 


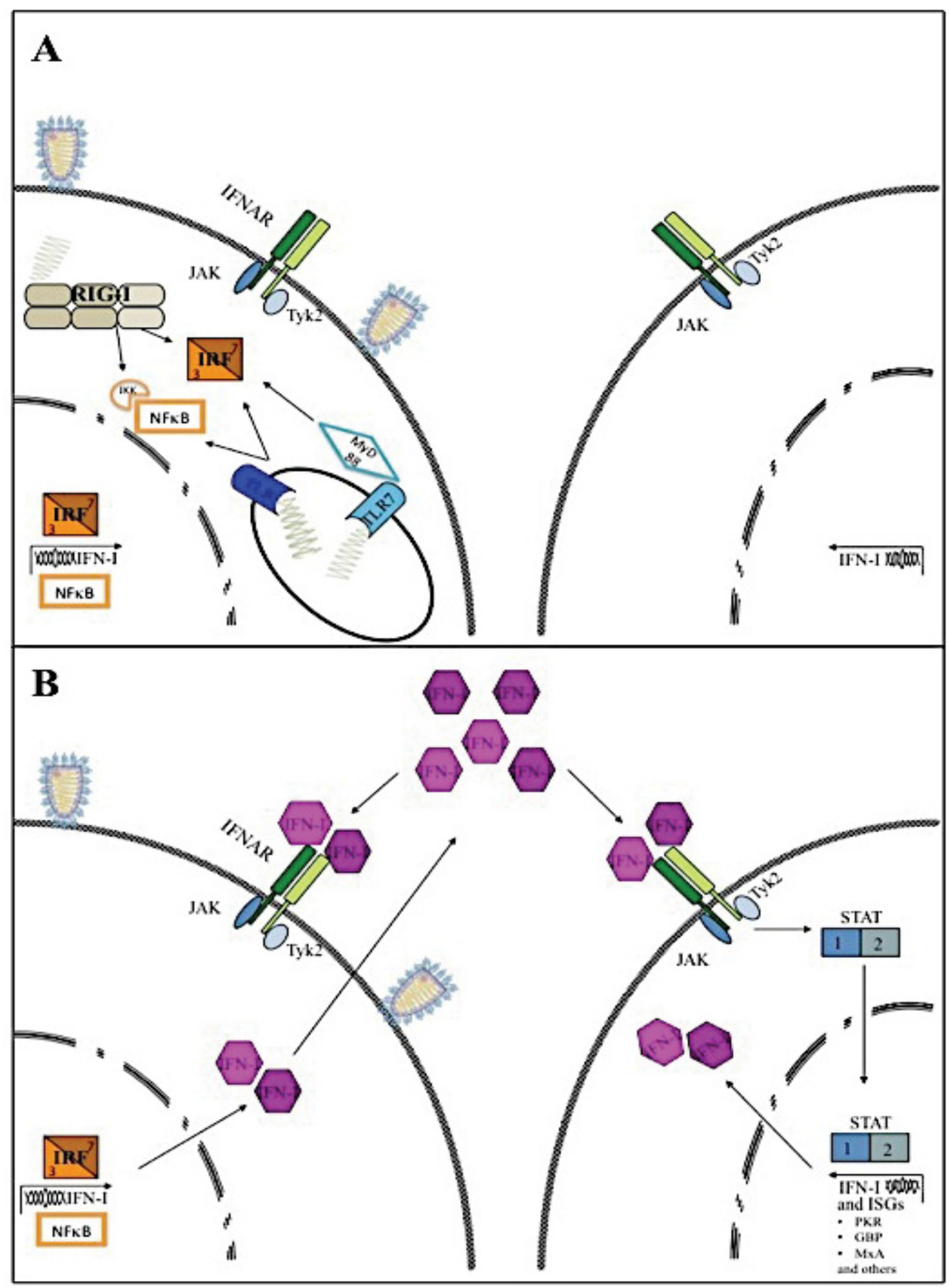




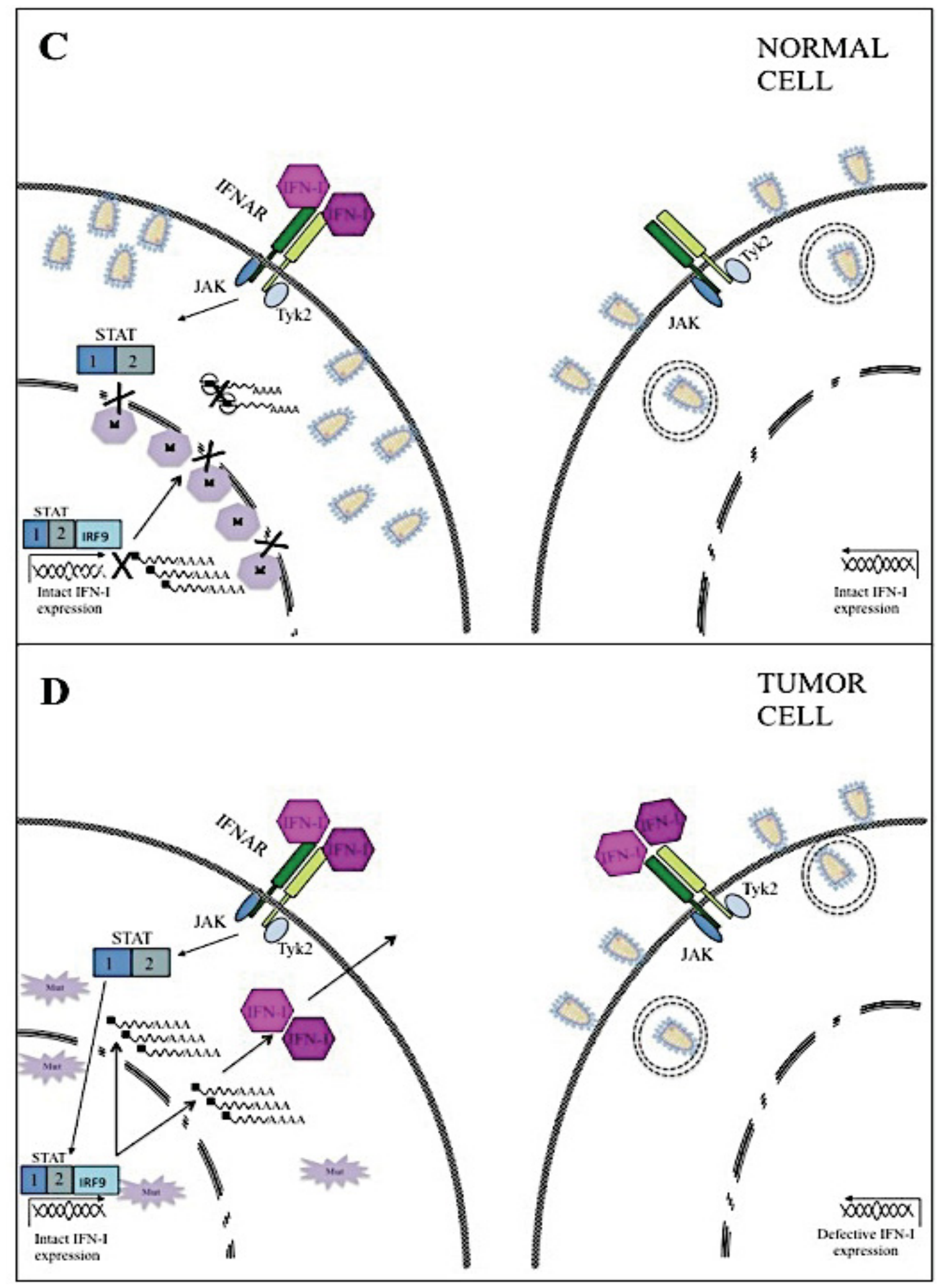

Figure 1-9. Continued 


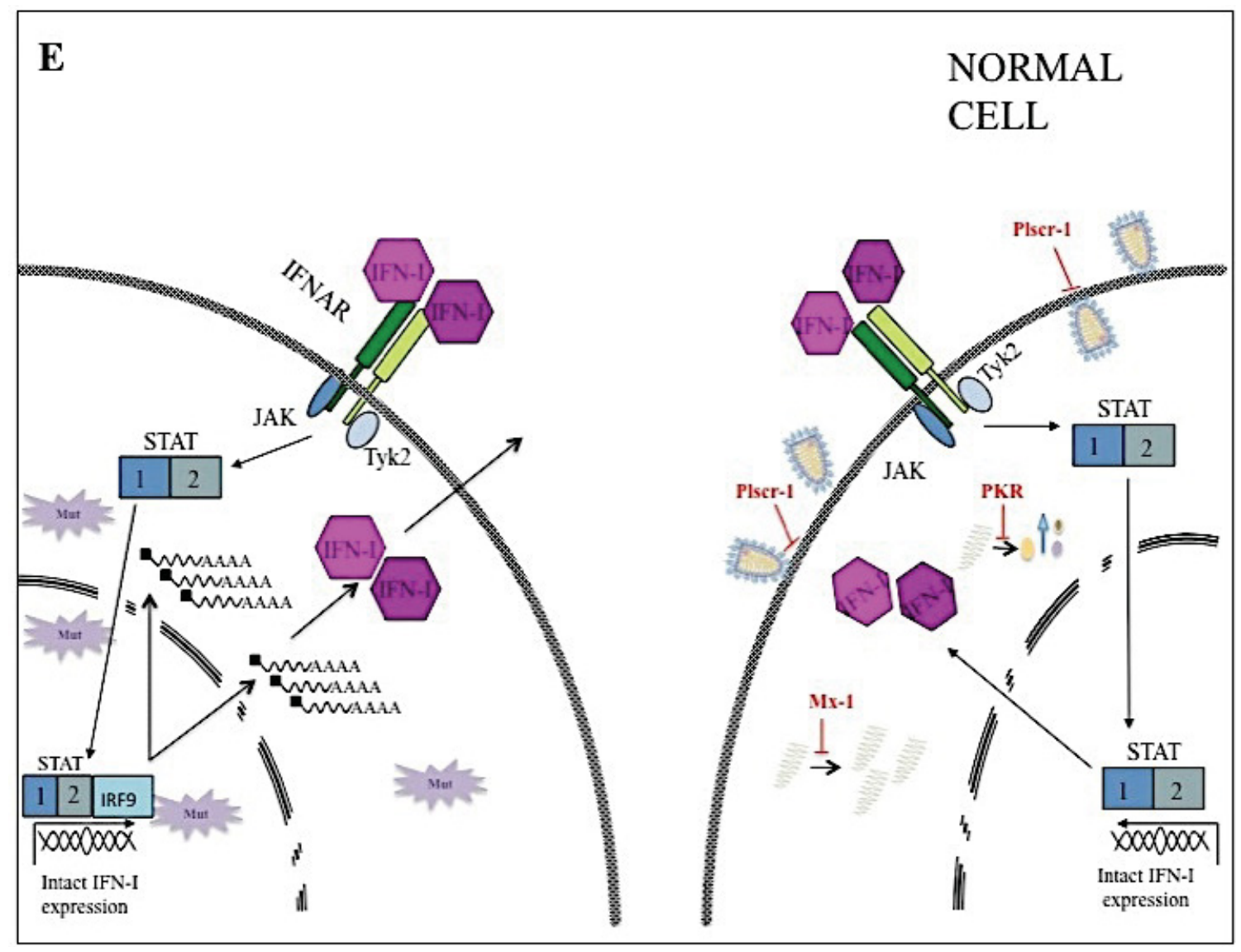

Figure 1-9. Continued 


\section{Pattern Recognition Receptors (PRRs)}

There are multiple ways in which viruses elicit downstream IFN expression. Initial responses involve recognition of pathogen associated molecular patterns (PAMPS) by pattern recognition receptors (PRRs) located in various compartments of the host cell. Membrane associated PRRs include Toll-like receptors (TLRs) while cytosolic PRRs include retinoic acid-inducible gene (RIG)-I-like receptors (RLRs), and the nucleotidebinding domain, leucine-rich repeat-containing (NBD-LRR) proteins (NLRs) (reviewed in [302]). Several PRRs are activated in response to VSV infection. These include TLR3, TLR7, and RIG-I (Figure 1-9A).

Upon binding of dsRNA replication intermediates, the endosome bound TLR-3 recruits adaptor molecule TIR-domain containing adaptor inducing IFN- $\beta$ (TRIF). TRIF activates downstream signals by two different cascades. The first works through phosphorylation of interferon regulatory factor 3 (IRF-3) and IFN regulatory factor 7 (IRF7), which promotes formation of an IRF3/7 heterodimer that migrates to the nucleus where it is able to bind to IRF-binding elements in the IFN- $\alpha / \beta$ promoter. In the second cascade, TRIF activates Inhibitor of kappa B kinase (IKK) complex, which leads to the dissociation of NF- $\mathrm{KB}$ from its inhibitor allowing its nuclear translocation, where it binds to and increases expression of IFN-I genes. Both, IRF3/7 and NF- $\kappa \mathrm{B}$ are required for the robust transcriptional activation of type I IFN during viral infection (reviewed in [302]). However, it has been shown in the case of VSV, that cells with silenced TLR3 still resist infection proving that there must be other vital pathways involved in anti-VSV responses [303]. For instance, TLR-7 is another endosome associated PRR found mostly in dendritic cells, especially plasmacytoid DCs (pDCs). TLR-7 predominantly binds ssRNA, which sparks recruitment of the adaptor protein myeloid differentiation factor 88 (MyD88). MyD88 activates IRF-7 downstream, which, again, forms a heterodimer with IRF3, promoting IFN expression in the nucleus. In comparison to TLR3, TLR7 is extremely important in antiviral signaling of VSV infection. Bone marrow cells derived from both MyD88 and TLR7 deficient mice expressed diminished levels of IFN $\alpha$ in response to VSV infection. This decrease in antiviral response was also observed in vivo as well [304].

Cytoplasmic PRRs have particular importance in eliciting IFN related antiviral

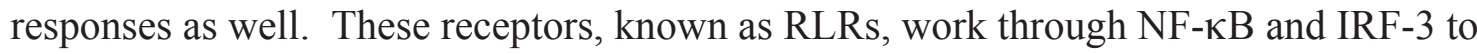
promote robust IFN-I responses. Retinoic acid-inducible gene-I (RIG-I) and melanoma differentiation-associated gene-5 (Mda-5) are two recognized RLRs important in RNA virus signaling however RIG-I is more important to VSV infection. RIG-I (also known as Ddx-58) recognizes 5'-triphosphates on viral RNAs, such as those encoded by negative- stranded viruses [305]. After detection, RIG-I activates the mitochondriabound interferon beta promoter stimulator-1 (IPS-1), which phosphorylates kinases, TANK-binding kinase 1 (TBK1) and IKKi that then activate IRF-3 and NF-кB, respectively (reviewed in [302]). The importance of RIG-I in host responses against VSV has been shown through experiments in which RIG-I knockout MEFs were unable to upregulate IFN- $\beta$ activity causing $10^{1.5}$ higher viral titers than in normal cells [306]. Alternatively, overexpression of RIG-1 inhibits replication of VSV in L929 cells by 
decreasing titers $10^{2-3}$ versus control cells [303].

\section{Interferon Stimulated Genes (ISGs)}

Following the initial induction of IFN-I genes, newly synthesized IFN act through paracrine and autocrine signaling to augment antiviral responses in a positive feedback loop (reviewed in [303]). Upon binding to its receptor, IFN ignites a phosphorylation cascade that results in activation of tyrosine kinases, Tyk2 and Janus kinase 1 (JAK1), both bound to the intracellular portions of IFNAR. Signal transducers and activators of transcription, known as STAT 1 and STAT2, are subsequently phosphorylated which promotes formation of a STAT $1 / 2$ heterodimer. The heterodimer is transported to the nucleus where it binds to IRF9, forming the IFN-stimulated gene factor 3 (ISGF3) complex that binds to the IFN-stimulated response element (ISRE) in the promoter of a multitude of interferon stimulated genes (ISGs) [296]. ISGs are the major operatives of the IFN pathway and over 300 ISGs are known to exist though most of their functions are not yet understood [307, 308]. Several have well-known IFN-mediated antiviral activities. These include the 2', 5'-oligoadenylate synthetase (OAS)/RNaseL pathway. OAS is triggered by dsRNA to activate RNase $\mathrm{L}$, which degrades both host and viral RNA. Another well-known ISG is the dsRNA-activated protein kinase (PKR), which responds to dsRNA by phosphorylating translation initiator factor eIF- $2 \alpha$ promoting cessation of protein synthesis. Lastly, the Myxovirus resistance (Mx) proteins, which will be discussed in more detail, are GTPases that prevent virus infection mostly at the transcriptional level (reviewed in [307]). Though important, OAS, PKR, and Mx are not the only ISGs involved in protection of cells against VSV. Following IFN-I pretreatment, triple (OAS, PKR, Mx) knockout MEFs still mounted an antiviral response against VSV infection [309] confirming that other ISG effectors are obviously playing some role in anti-VSV responses and may include broadly acting effectors such as IRFlor more specific effectors such as guanylate binding proteins (GBPs).

\section{Interferon regulatory factor 1}

Interferon regulatory factors (IRFs) are a family of transcription factors made up of nine members in humans and mice [310]. The first of these identified was IRF-1, initially shown to activate IFN-I in the absence of viral infection. Though IFN-inducible, IRF-1 is constitutively active in a number of cells with levels dependent upon the cell cycle, which are increased during cell cycle arrest [311]. Serving as a transcription factor is IRF-1's main mechanism of action and inactivating the ability to bind to DNA eliminates its activity [312]. IRF-1 protein is mainly located in the nucleus where it is known to not only increase expression of IFN but other ISGs as well including OAS, PKR, and GBP. It specifically recognizes a sequence in the promoter region very similar to that recognized by the ISG3 complex therefore, it is thought that these two activators of IFN also activate a similar subset of ISGs [310]. Because IRF-1 is itself induced by viral infection, de novo synthesis of IRF-1 is believed to be important in eliciting IFN responses [313]. Though IFN-I can cause upregulation of IRF-1, its strongest inducer is 
IFN- $\gamma$ therefore a number of additional stimulated genes involved in other pathways including apoptosis and anti-proliferation is also activated by IRF-1 [310].

Aside from its role in antiviral responses, other roles of IRF-1 include antitumor activity [314] and modulation of the immune system, including adaptive responses involving sculpting the antigen specificity of CD8+ T cells [315]. Studies involving IRF1 knockout MEFs demonstrated IFN-I continued to be upregulated following infection with Newcastle disease virus though upregulation with polyI:C was impaired (reviewed in [310]).

\section{Myxovirus resistance protein}

Myxovirus resistance $(\mathrm{Mx})$ proteins are members of the dynamin-like GTPase family of interferon stimulated genes [316]. There are two Mx proteins encoded by two separate genes in humans while there are 3 separate Mx genes in rats. Human MxA is a cytoplasmic protein active against a number of RNA viruses including VSV. Cells that constitutively express MxA experience a decrease in viral transcription by 50 fold [317]. Alternatively, MxB has not been shown to have any antiviral activity [316]. In rat, Mx1 is found in the nucleus where it inhibits nuclear replicating viruses such as influenza, while the cytoplasmic Mx2 is more effective against viruses that replicate in the cytosol such as VSV. Mx3, like human MxB, has no known antiviral activity [318]. Mx protein activity is thought to attributed to the ability to sense nucleocapsid like structures and prevent them from participating in replication in the nucleus, in the case of Mx1, or in the cytoplasm, in the case of Mx2 [318].

\section{Guanylate binding protein}

Guanylate binding proteins or GBPs are highly conserved proteins belong to the dynamin-like family of GTPases, like Mx proteins. There are seven members of the human GBP family [319] and 11 known murine GBPs [320]. GBPs are cytoplasmic proteins. Specifically, mGBP-2 has been shown to localize to intracellular vesicle-like structures although the importance of this localization on function has not yet been described [321]. GBP promoters contain several ISRE's that are regulated by other ISGs such as IRF1 [322]. GBPs demonstrate antiviral activity, though weak in comparison to the antiviral responses of Mx proteins [322]. Particularly, HeLa cells stably transfected with hGBP-1 exhibited resistance to VSV-induced CPE, making less virus than control

cells. Cells with silenced expression of hGBP1 that were treated with IFN $\alpha$ produced the same amount of virus as cells with normal hGBP1 levels however these same cells pretreated with IFN- $\gamma$ had increased viral replication and less antiviral responses than parent cells. This demonstrates that though GBP is responsive to IFN-I, upregulation is more important in IFN-II signaling [323].

Outside of its role in antiviral responses, GBPs are implicated in having an antitumor effect. Adding to the above results, the overexpression of mGBP2 enhances 
proliferation in NIH3T3 cells similar to the effects of IFN- $\gamma$ while the opposite holds true for hGBP1 and IFN- $\gamma$ in HeLa cells which have anti-proliferative effects in this cell line [324]. Further, hGBP1 and hGBP2 were recently shown to be upregulated in EGFR activated cells, causing a downstream induction of matrix metalloproteinase-1 (MMP-1) driving invasion of GBM cells making it a novel target in treatments of GBM [325].

\section{Phospholipid scramblase 1}

Phospholipid scramblase 1 (Plscr1) is another ISG that has been identified as a possible IFN-induced antiviral component in VSV infection [326]. Plscr1 is a calcium binding protein whose location is dependent on its state of palmitoylation. It can either be found in the nucleus bound to DNA or in lipid-raft-associated plasma membrane, promoting calcium induced bidirectional movement of phospholipids [327]. The N terminal cytoplasmic domain is proline-rich and likely serves as a binding motif for other proteins containing SH3 and WW domains [328]. Based on location, plscr1 antiviral activity is believed to involve inhibition of viral adsorption and enhancement of IFN signaling at the plasma membrane. Cells with decreased Plscrl activity are insensitive to the antiviral effects of IFNs and are more sensitive to VSV infection, confirmed by elevated viral titers relative to wildtype cells. Plscr1 is known to upregulate the expression of a number of other ISGs including GBPs, OAS, RNase L, and PKR [329].

\section{Defective IFN Responses}

There are a number of ways that tumor cells can bypass the anti-proliferative effects of IFN. These include acquired defects in the ability to make IFN (i.e. chromosomal aberrations) and to respond to IFN (i.e. alterations in signaling cascade). As has been described, many tumors have deletions in chromosome 9 in the location of the IFN-I gene cluster. Chromosome 9 aberrations are commonly encountered in GBM, and allow cells to escape growth arrest and induction of apoptosis [330]. The variable nature of IFN resistance was acknowledged in a comparison between two GBM cell lines, one known to have homozygous deletions in chromosome 9 and another without chromosomal defects [23]. Both were equally resistant to the IFN $\alpha$-induced apoptosis. This raises the importance of non-chromosome 9 abnormalities that may lead to IFN resistance including dysregulation of IFN regulatory factors and other interferon inducible genes [331]. In an evaluation of STAT1 expression in GBM tumors, it was shown that tumors expressing STAT more likely expressed an inactive form located in the cytoplasm versus the active, nuclear form [332]. Other IFN pathway constituents that have been implicated in tumorigenesis include IKK complex, important in activation of NF-kB and the interferon regulatory factor, IRF1 [333, 334]. On a more global scale, downstream IFN effects on p53 signaling have been implicated in disruption of antiviral responses in tumor cells. IFN can activate expression of p53 through an ISRE found in its promoter and as we know, p53 is a regulator of cell cycle and one of the most common targets of mutation in cancer [300]. 


\section{Other Viral Antagonists of the IFN Pathway}

Tumor selective targeting of the IFN pathway is not unique to VSV. In addition to blocking host gene expression, other viral mechanisms used to escape immune responses include sequestering IFN or IFN related responders and by cleaving or even degrading antiviral products of the IFN pathway. There are over 170 virus-encoded IFNantagonists known in 93 different viruses, some highly conserved among various RNA families (reviewed in [335]). An example is the conserved V proteins of the Paramyxoviridae family in the order Mononegavirales. Particularly, V proteins prevent dimerization of the cytosolic PRR, MDA-5, and also target STAT for proteasomal degradation [336, 337]. The highly conserved Z proteins of New World Arenaviruses antagonize antiviral responses by binding to and inhibiting RIG-I activity [338]. Comparable to VSV matrix, the non-structural NS1 proteins of influenza virus are multifunctional proteins with multiple mechanisms for antagonizing the IFN pathway. Just as matrix, NS1 can inhibit host gene expression by blocking RNA export. NS1 can also bind to dsRNA which prevents activation of the OAS/RNase L pathway and inhibits transcription of antiviral genes by preventing dsRNA-dependent transcription factors from being activated [339]. Coronaviruses use its non-structural protein, Nsp1, to degrade cellular mRNA, a nonspecific mechanism for blocking antiviral gene expression [340]. Specifically, SARS coronavirus utilizes the accessory protein, ORF6 to inhibit transport of STAT1 to the nucleus by blocking nuclear transport protein, preventing the feedback loop vital for augmenting IFN responses [341]. Lastly, rabies virus, another member of the Rhabdoviridae family, is also able to inhibit antiviral responses during infection. Unlike VSV, however, rabies uses its phosphoprotein (P) to prevent phosphorylation of the regulatory factor IRF-3 as well as inhibit STAT1 signaling. Rabies takes a targeted multistep approach to antagonizing IFN whereas VSV inhibition is more global [342].

\section{Advantages of VSV Oncolytic Therapy}

As with most OVs, there are both advantages and disadvantages of using VSV as an oncolytic vector. The first advantage is that VSV is a naturally tumor selective virus, having a replication advantage in tumor cells that harbor defects in the IFN pathway [294]. Even more, VSV can be easily manipulated using reverse genetic techniques to enhance tumor selectivity as demonstrated by the genetically engineered matrix mutants. Virions can also be engineered to express foreign genes, accommodating up to $4.5 \mathrm{~kb}$ of foreign RNA without significantly altering replication and viral gene expression [207]. A more recent study reported the insertion of the hepatitis C NS protein measuring approximately $5.9 \mathrm{~kb}$ without affecting replication [343]. Other examples include VSV vectors expressing immune enhancing molecules such as IFN $\beta$ or tumor associated antigens that boost antitumor immunity [344, 345]. For instance, a therapeutic regimen using a VSV vector expressing the surrogate tumor antigen, ovalbumin, utilized a virolytic and immunotherapeutic approach for the treatment of ova expressing B16 melanomas in a mouse model. Results showed enhancement of immune activation against ova by increasing tumor antigen specific CD8+ T cells, which aided in 
enhancement of viral oncolysis as well [346].

The fact that VSV is not considered a human pathogen is also a benefit of VSV oncolytic therapy. Seroconversion is rarely seen in the human population so the risk of pre-immunity is extremely low [207]. Even in cases of pre-immunity, as has been observed in cattle after natural VSV infection, immunity is not durable and animals may be re-infected with the same VSV strain in subsequent years despite high serum neutralizing antibody titers [213]. Whereas DNA and lentiviral vectors run the risk of insertional mutagenesis due to integration into the host genome, the use of RNA viruses such as VSV makes insertional mutagenesis highly unlikely. Lastly, the quick replication cycle makes it easier to produce large amounts of virus and its broad host range facilitates its therapeutic use in a multitude of tumors [291]. Viral yields in baby hamster kidney cells (BHK-21) are about 100,000 particles per cell, of which about 10\% can be infectious [207]. Other cell types that have been tested include kidney, brain, and heart with titers equaling $10^{7}, 10^{6}$, and $10^{5}$ respectively [346].

\section{Disadvantages of VSV Oncolytic Therapy}

On the other hand, there exists a number of disadvantages to VSV oncolytic therapy. First, as with other RNA viruses, VSV has a high rate of mutation, with RdRp errors occurring every 1:10,000 nucleotides or approximately 1 mutation/virion [347]. As a result, the risk of reversion to wildtype will continue to be a factor as VSV moves from bench to bedside. Previous studies using one of the more popular VSV mutants, $\mathrm{M} \Delta 51$, has shown that the possibility of reversion, though present is extremely rare in this case. It is proposed that when using this particular IFN inducing vector, the likelihood of a wildtype revertant rising to dominance would be virtually impossible [289].

VSV along with several other viruses such as rabies, HSV, and measles, are known to be neurotoxic [348-350]. Evidence for VSV neurotoxicity stemmed from studies in which mice were experimentally challenged with virus given either intranasally or intracranially. Understanding the neurotoxic phenotype of VSV is important specifically in developing VSV as an oncolytic treatment for brain tumors as protection of normal cells is crucial [219, 351].

One study initially aimed at determining ways of lessening neurotoxicity resulted in uncovering what can be considered a disadvantage, though a necessary one, of VSV therapy, the adaptive immune response. As discussed, with natural infection, preimmunity does not protect animals, long term, against subsequent infection with the same serotype [213]. However, studies using experimentally infected animals revealed that long lasting immunity is attainable following i.v. challenge with either wtVSV or attenuated viruses (VSV-M51-CT9, VSV-CT1; CT mutants are defined as having truncated cytoplasmic domains of the VSV glycoprotein) [220]. These differences may be attributed to variations in routes of administration and species of infected animals, however adaptive immunity still serves as a hurdle to efficient delivery of viral therapy, therefore, understanding the natural progression of adaptive immunity is important. 
The timeline leading to the activation of adaptive immune responses has been determined using a mouse model. Within one week of infection, neutralizing immunoglobulin M (IgM) antibody appears first followed by appearance of neutralizing IgG antibodies (nAb). Strong adaptive responses including antibody and cytotoxic Tlymphocytes are directed against $\mathrm{N}$ and $\mathrm{G}$ proteins [352] however, nAbs are specifically directed towards the $\mathrm{G}$ protein. Further, passive immunity with $\alpha \mathrm{G}$ antibodies, but not antigen specific T cells, protect against systemic infection (reviewed in [207]). The importance of adaptive immune effectors was revealed in studies using immunodeficient animals. B-cell-deficient mice are highly sensitive to VSV and usually succumb to infection within 9 days. On the other hand, T cells contribute to long-term survival as Tcell-deficient mice succumb to neurotoxicity closer to 30 days post- infection [352]. In creating a safe and effective VSV therapy that can be moved from preclinical to clinical studies for the treatment of GBM, a balance must be met between lessening neurotoxic effects of viral infection and achieving optimal delivery with long lasting therapeutic effects.

\section{Examples of VSV-Derived OVs in Preclinical and Clinical Trials}

Several VSV-derived OVs have been well characterized with studies confirming increased safety and efficacy over wildtype virus [289] (reviewed in [68]) (Table 1-5). Vectors of interest possess mutations ranging from the phosphoprotein (VSV-rp30a) [353], glycoprotein (VSV $\Delta \mathrm{G}$ ) [354], and matrix protein (M51) (reviewed in [355]). I will discuss several mutants that have been more heavily studied in preclinical and more recently, in clinical trials, though not for GBM.

\section{AV1 and AV2}

AV1 (attenuated virus 1) and AV2 (attenuated virus 2) are interferon-inducing VSV mutants originally identified by their small plaque size in non-IFN defective cells and large plaques in IFN defective cells [356]. These constructs are based on mutations found in two temperature sensitive mutants, T1026R and TP3, respectively [357]. AV1 contains the classical M51R matrix mutation where AV2 contains two additional mutations V221F and S226R. Just as previous studies have shown that, M51R mutants are defective specifically in their ability to inhibit NCT of IFN message, AV viral mutants were also found to have the same phenotype, with IFN- $\beta$ mRNA found in both the nuclear and cytoplasmic pool of mRNAs in culture media from AV-infected cells [289]. A panel of tumor cells ranging from breast cancer to colon cancer were tested and found to be highly susceptible to infection with these attenuated viruses. To specifically test attenuation in animals, AV's were compared to wildtype virus in an immunocompromised PKR-/- mouse model. AV's were tolerated up to a $10^{7} \mathrm{pfu}$ (plaque forming units) dose while only 10 pfu of wildtype caused death in these animals. Several

other studies have been carried out in tumor bearing animals, specifically using the single matrix mutation found in AV1. In animals harboring lung tumors, systemic and intranasal administration of AVs resulted in an increase in survival relative to UV- 
Table 1-5. VSV-derived oncolytic viruses for the treatment of GBM

\begin{tabular}{|c|c|c|c|}
\hline VSV Vector & $\begin{array}{l}\text { Oncolytic } \\
\text { Mechanism }\end{array}$ & $\begin{array}{l}\text { Viral Protein/ } \\
\text { Mutation(s) }\end{array}$ & References \\
\hline AV1 & $\begin{array}{l}\text { Defects in IFN } \\
\text { pathway }\end{array}$ & Matrix/M51R & {$[289,356-359]$} \\
\hline AV2 & $\begin{array}{l}\text { Defects in IFN } \\
\text { pathway }\end{array}$ & $\begin{array}{l}\text { Matrix/ M51R, } \\
\text { V221F, S226R }\end{array}$ & {$[289,356,357]$} \\
\hline VSV-M $\Delta 51(\mathrm{AV} 3)$ & $\begin{array}{l}\text { Defects in IFN } \\
\text { pathway }\end{array}$ & $\begin{array}{l}\text { Matrix/ M51 } \\
\text { deletion }\end{array}$ & {$[187,193,285,289]$} \\
\hline VSV-rp30a & $\begin{array}{l}\text { Selective adaptation } \\
\text { in glioma cells }\end{array}$ & $\begin{array}{l}\text { Phosphoprotein/ } \\
\text { not reported } \\
\text { Large } \\
\text { polymerase/ } \\
\text { not reported }\end{array}$ & {$[353,355,360]$} \\
\hline VSV-IFN $\beta$ & $\begin{array}{l}\text { Defects in IFN } \\
\text { pathway }\end{array}$ & Insertion of IFN $\beta$ & {$[344,361-363]$} \\
\hline
\end{tabular}


inactivated virus. Tumor load was also reduced as tumors treated with UV-inactivated virus reached a volume of $750 \mathrm{~mm}^{3}$ by 10 days post-implantation whereas AV treated tumors never surpassed approximately $200 \mathrm{~mm}^{3}$ throughout the study [289]. Another study demonstrated the variability in response of tumors of the same type to VSV oncolysis in vivo. Animals harboring subcutaneous prostate tumors were either treated intra-tumorally or systemically with wt-VSV or M51 mutant. In one prostate tumor ( $\mathrm{LNCaP})$, both viruses caused tumor reduction however in the other prostate tumor (PC3), cells remained resistant and grew at the same rate as untreated tumors [358]. Lastly, an evaluation of M51R mutant treatment against human gliomas was carried out in a xenograft mouse model of subcutaneously implanted U87 tumors. This study revealed that M51R mutant completely eliminated tumors within 21 days post-infection without viral induced morbidity whereas untreated animals reached euthanasia criteria as a result of tumor load by 1 week [359]. To date, no clinical studies have been carried out for either of these attenuated viruses.

\section{VSV-M $\Delta 51$}

VSV-M $\Delta 51$, also known as AV3 when expressing a fluorescent reporter gene, contains a single amino acid deletion in matrix at position 51. Initial in vitro studies tested the susceptibility of primary gliomas and glioma cell lines to infection, which were all found to be highly susceptible to oncolysis by the mutant vector [289]. In safety and efficacy studies using xenograft models of U87 and U118 human malignant glioma in nude mice, intratumorally and intravenously administered virus markedly reduced tumors compared to UV inactivated treated animals. Intravenous administration of VSVAM51 was found to significantly prolong survival in mice with unilateral U87 tumors (median survival of 113 versus 46 days) and bilateral U87 tumors ( 73 versus 46 days). No toxicity was observed in this study. Therefore, VSVAM51given systemically was able to migrate to intracranial tumor and decrease tumor load while prolonging survival in this model [285].

A subsequent study using the GFP expressing VSV $\triangle$ M51 construct, AV3, in an immunocompetent model of lung cancer sought to determine if the adaptive immune responses which have been shown to attenuate VSV oncolysis could be circumvented by using cell carriers, a novel mode of systemic administration. AV3 infected cells were able to shield viral antigen during transport to tumor site where they then released virus, infecting malignant cells and not surrounding normal tissue. Repeat administration of infected cells improved therapeutic efficacy when compared to naked virion injection with durable viral replication observed up to 6 days post-treatment. Two cell carriers, tumor derived cells and leukemic cells, were tested for their ability to migrate to tumor following i.v. administration. Tumor carriers were found to localize in the lungs whereas leukemic carriers migrated to the lungs and throughout the body [187].

Whereas most studies are based on the premise that VSV selectively targets tumors due to IFN defects, not all tumors fall into this category, as was described with VSV-resistant prostate tumors. There are also groups of malignant gliomas that have 
been found to have active IFN pathways resulting in less responsiveness to VSV therapy. Some of these tumors harbor defects in mTORC1 pathway, a pathway that signals IFN activation $[364,365]$. A recent study tested the use mTORC1 inhibitor, rapamycin, as an adjuvant to VSVDM51 in vitro and in an immunocompetent intracranial rat glioma model to determine enhancement of antitumor effect. By reducing IFN activity, combination therapy significantly increased survival while specifically targeting and reducing intracranial tumors [193]. As with the other AV constructs, there are no ongoing or previous clinical trials for VSVAM51.

\section{VSV-rp30a}

Unlike most VSV therapies, VSV-rp30a is a naturally occurring mutant derived through repetitive passage in glioma cells whose evolutionary pressure led to tumor specific mutations that enhance viral fitness in glioma cells [353]. Its increased fitness is attributed to two confirmed mutations, one in the $\mathrm{P}$ protein and the other in the $\mathrm{L}$ protein of VSV [355] however the exact mutational changes in these proteins have not been

revealed. In vitro studies comparing the wildtype virus to the glioma-adapted VSV-rp30a demonstrated a replication advantage of both viruses in human glioblastoma cell lines over non tumor cells marked by increased cell rounding and decreased cell viability. As expected, VSV-rp30a displayed increased growth ability in tumor cells relative to wildtype at earlier time points. Following IFN- $\alpha$ or polyI:C pre-treatment, some protection was observed at early time points in glioma cells with complete protection in normal cells. These results corresponded to MxA expression levels following either viral infection or pre-treatments, with a boost in MxA observed in all normal cell types and varying levels in glioma cells ranging from little to no expression. This suggests the variable nature of IFN defects in GBM cells which may explain differences in susceptibility to viral oncolysis in different tumors [353].

In an immunodeficient, xenograft mouse model of human intracranial and metastatic glioma, VSV-rp30a was shown to not only target intracranial tumors but also migrated to sites of extra-cranial tumors as well. Though longer time points were not included in this study in order to determine differences in survival and tumor load reduction, it was shown that virus effectively replicated in transplanted tumors at up to 72 hours post-infection and that several glioma tumors possessing different genetic aberrations in the p53 pathway were equally susceptible $[63,360]$. As of yet, no clinical trials are planned for VSV-rp30a.

\section{VSV-IFN $\beta$ (mIFN $\beta$, mouse IFN $\beta$; $\operatorname{rIFN} \beta$, rat IFN $\beta$; hIFN $\beta$, human IFN $\beta$ )}

Since the IFN pathway is the most accepted proposed mechanism for selective replication of VSV in tumor cells leading to oncolysis, a VSV construct expressing IFN $\beta$ (VSV- IFN $\beta$ ) was developed. It was originally tested for treatment of hepatocellular carcinoma (HCC) but has since been evaluated as treatment for multiple myeloma (MM). In vitro, VSV-hIFN $\beta$ has been shown to have a non-lytic phenotype in normal human 
cells while remaining oncolytic in malignant cells [344]. To verify safety in animals, intravenous and intranasal VSV- mIFN $\beta$ was administered in immunocompetent mice and was shown to be significantly attenuated compared to wild-type VSV [344], however subsequent studies demonstrated neurotoxicity when given intravenously causing fatal encephalitis in inoculated animals [361]. Additional studies have evaluated VSVIFN $\beta$ for treatment of HCC and MM. To first determine safety and MTD, immunocompetent Sprague Dawley rats and rhesus macaques were administered intrahepatic VSV- hIFN $\beta$ with no adverse events appreciated. However, in an orthotopic syngeneic model of HCC in Buffalo rat, intratumoral administration caused neurotoxicity with VSV- hIFN $\beta$ construct that lessened by using species-specific rVSV-rIFN $\beta$. No results of tumor load reduction were determined in this study [361]. In studying VSVhIFN $\beta$ as a treatment for multiple myeloma, significantly prolonged survival and antitumor activity was demonstrated in an immunocompetent mouse model of subcutaneous and disseminated myeloma. Species-dependent responses were also observed in this study, as mIFN $\beta$ construct prolonged time to progression in the mouse model over the use VSV-hIFN $\beta$ [362]. A phase I trial at Mayo clinic testing VSV- hIFN $\beta$ for treatment of adult primary HCC or recurrent primary liver cancer is in progress and planned to be complete by June 2013 [363].

As with OVs derived from different viruses, there can be differences in the oncolytic profile of alternate constructs derived from the same virus. Each may possess their own advantages and disadvantages, therefore, an understanding of underlying tumor biology of the cancer type being treated is important. Van den Pol and associates compared several VSV constructs in their potential as a treatment for GBM. Of those tested, VSV-M51, VSV- CT9-M51 (CT9- truncation of G protein cytoplasmic tail), VSV-p1-GFP (insertion of GFP at the 3' end of the genome), and VSV-p1-RFP (insertion of red fluorescent protein (RFP) at the 3 ' end of the genome) were safest and most effective at destroying malignant glioma while other constructs including VSV- $\Delta \mathrm{G}$ and VSV-rp30a were least effective [355]. These may prove to be the best vectors to consider for phase trials in the treatment of GBM as newer more tumor selective constructs become available.

\section{rNCP12.1}

rNCP12.1 (recombinant Non-CytoPathic isolate 12.1) is a novel attenuated replication competent VSV vector developed by Whitt and associates due to its noncytopathic effects in a number of cell lines [237]. It was originally isolated after establishment of a persistent infection in BHK-21 cells with another M mutant, M33, 51A. As discussed, due to the mutations at positions 33 and 51 in the matrix protein, this mutant is unable to express the truncated matrix products, M2 and M3. In effect, M33, 51A mutant is defective in cell rounding especially in BHK-21 cells while maintaining wildtype cytotoxicity in several other cell types, particularly HeLa and 293 cells. No defects in host cell shutoff were appreciated for this mutant [237]. Because M33, 51A causes only $\sim 40-50 \%$ of cells to round following infection [237], cells that remained flat were further cultured to maintain a persistent infection as confirmed by viral expression 
of an inserted green fluorescent protein and from viral titers. Resultant virus from this persistent infection was found to have two mutations in the matrix protein in addition to M33,51A. These additional mutations are located in the C-terminus of matrix and correspond to T133A and S226G. The matrix of the original non-cytopathic variant, NCP12, was subcloned into a wt background and this recombinant vector was designated rNCP12.1 [237].

Whereas M33, 51A causes approximately half of BHK21 cells to round following infection, rNCP12.1 cell rounding is negligible in BHKs. However, in other transformed cell types such as HeLa and HEK293 cells, cell rounding is present but variable [366]

(Figure 1-10). Therefore it was implied that the cell rounding phenotype of rNCP12.1 is cell type specific and may depend on the presence of some host factor. Further characterization revealed that, like M33, 51 A, host cell shutoff was similar to wtVSV at 8hpi. However, unlike wtVSV, viral gene expression was decreased especially for M and G proteins, which could be due to an overall increase in antiviral host response [366]. In keeping with other studies that support the genetic separation of domains involved in budding and assembly from those involved in CPE [242] rNCP12.1 budding, though slightly decreased from wtVSV, maintained efficiency. Viral yields were approximately 10 fold less than wt at 18 hours post infection (hpi). Of the four mutations in the matrix of rNCP12.1, position M51 is the most important in its overall phenotype. M33A and T133A alone displayed CPE similar to wt whereas S226G expressed intermediate cell rounding but yielded virus similar to wt levels. M51A alone made 10 fold less virus than wt. More than likely, the overall phenotype of NCP12.1 is due to an additive effect of the combination of these mutations (Table 1-6).

This phenotype has been exploited in our lab in several ways. First, because of its decreased cytotoxicity in normal cells coupled with its near normal assembly and budding function, rNCP12.1 was used to recover a recombinant VSV vector lacking the $\mathrm{M}$ gene ( $\triangle \mathrm{M}-\mathrm{PLF})$. Until this time, recovery of VSV vectors lacking $\mathrm{M}$ were impossible due to the inhibition of gene expression caused by expression of wt $\mathrm{M}$ needed for complementation [366]. In addition, its use has been important in developing an oncolytic virus for the treatment of glioblastoma. To this end, preliminary results have been promising and will be described in further detail in this dissertation. 


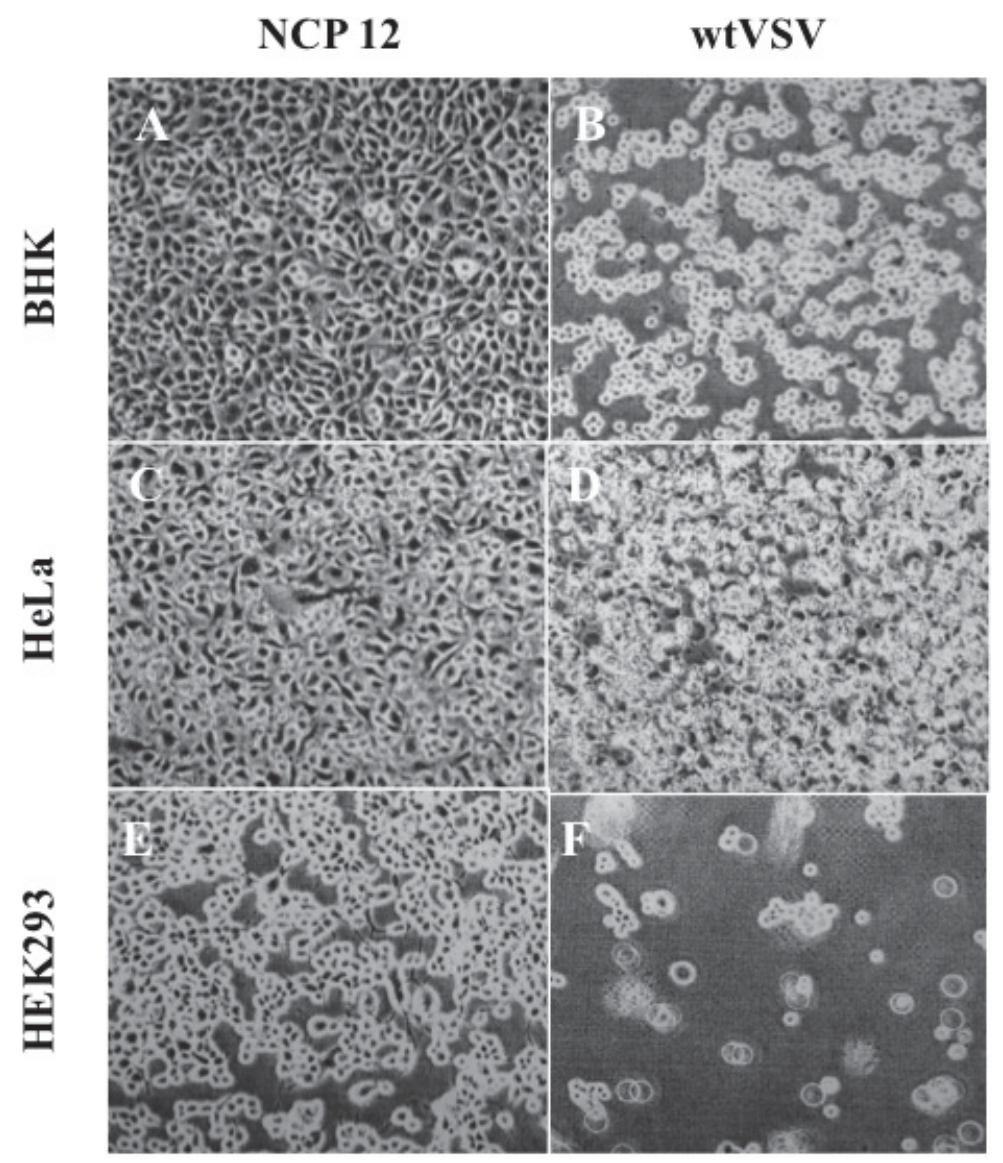

Figure 1-10. Cell rounding phenotype of rNCP12 in different cell types

rNCP12 is the parent virus of rNCP12.1. Phase contrast micrographs, taken at $10 \mathrm{X}$ magnification, show differences of cell rounding by NCP12 and wtVSV infection in (A, B) BHK cells, (C, D) HeLa cells, and (E, F) HEK 293 cells at 24hpi.

Reprinted with permission: H.R. Jayakar, VSV M protein domains involved in assembly and cytopathogenesis, 2001 [366]. 
Table 1-6. Characterization of individual mutations in matrix mutants

\begin{tabular}{|c|c|c|c|c|}
\hline Virus & Mutation & Virus Yield $^{\mathrm{a}}$ & $\begin{array}{c}\text { Cell } \\
\text { Rounding }^{b}\end{array}$ & Host Shut Off \\
\hline Wild-type & None & +++ & +++ & +++ \\
\hline M33A & $\mathrm{M} 33 \rightarrow \mathrm{A}$ & +++ & +++ & ND \\
\hline M51A & $\mathrm{M} 51 \rightarrow \mathrm{A}$ & + & + & ND \\
\hline M33,51A & $\begin{array}{l}\mathrm{M} 33 \rightarrow \mathrm{A} \\
\mathrm{M} 51 \rightarrow \mathrm{A}\end{array}$ & +++ & + & +++ \\
\hline T133A & $\mathrm{T} 133 \rightarrow \mathrm{A}$ & +++ & ++++ & ND \\
\hline S225G & $\mathrm{S} 225 \rightarrow \mathrm{G}$ & +++ & ++ & ND \\
\hline rNCP12.1 & $\begin{array}{l}M 33 \rightarrow \mathrm{A} \\
\mathrm{M} 51 \rightarrow \mathrm{A} \\
\mathrm{T} 133 \rightarrow \mathrm{A} \\
\mathrm{S} 225 \rightarrow \mathrm{G}\end{array}$ & + & - & + \\
\hline
\end{tabular}

a +++ indicates wildtype levels of virus titers $(\sim 5 \times 108-1 \times 109)$, and + indicates atleast 10 fold reduction in yield

${ }^{b}+++$ indicates $>90 \%$ of cell rounding in BHK21 cells by $24 \mathrm{hpi}$, and + indicates $<50 \%$ rounded cells; - indicates no cell rounding

${ }^{\mathrm{c}}+++$ indicates $>50 \%$ host cell shutoff by $8 \mathrm{hpi}$; + indicates $<50 \%$ host cell shutoff $\mathrm{ND}$, not done 


\section{CHAPTER 2. MATERIALS AND METHODS}

\section{Cells and Viruses}

All cells were maintained as a monolayer culture at $37^{\circ} \mathrm{C}, 6 \% \mathrm{CO} 2$. All medium was supplemented with 100U/mL of penicillin and streptomycin (Table 2-1). The rat F98 and C6 glioma and human U87 glioma cell lines were previously transduced with the pFB retrovirus (pFB-GFP) (Stratagene, La Jolla, CA) expressing GFP to allow visualization of tumor load in brain sections. Cells stably transduced with GFP were sorted using flow cytometry to generate a cell population homogeneously expressing high levels of GFP.

All recombinant VSV constructs were made in the Lab of Michael A. Whitt, $\mathrm{PhD}$ and contained genes of the Indiana serotype with the exception of the glycoprotein of the New Jersey serotype $\left(\mathrm{G}_{\mathrm{NJ}}\right)$ for rNCP12.1 $1_{\mathrm{NJ}} \mathrm{GFP}$ (Table 2-2).

\section{Cell-Rounding Assay}

Rat glioma (C6, F98), human glioma (U87, A172, and T98G), and PRAs were plated in 35-mm-diameter dishes and infected with rNCP12.1-GFP (NCP12.1-dsRed in U87, C6, F98) at an MOI of 10 (rat glioma) and MOI of 1 (human glioma) for one hour at $31^{\circ} \mathrm{C}$. Inoculum was removed, and the cells were washed once with serum-free medium and then incubated at $37^{\circ} \mathrm{C}$ for varying times. At $24 \mathrm{hpi}$, medium was removed, and cells were washed twice with phosphate- buffered saline (PBS) and fixed with 3\% paraformaldehyde at room temperature for $20 \mathrm{~min}$, followed by two washes with PBS containing $20 \mathrm{mM}$ glycine. Cells were observed by bright field and fluorescence microscopy (Zeiss, Axiovision) to determine presence of viral and tumor cell fluorescent markers.

\section{Growth Kinetics}

Rat glioma cells and PRAs were seeded into 96 well plates for 24 hours then mock infected by incubation in serum free media or infected with either wt-VSV or rNCP12.1-GFP for one hour at $31^{\circ} \mathrm{C}$ at an MOI of 10. Inoculum was removed and cells were washed with serum free (SF) DMEM three times. Fresh D10 was added to all cell lines and incubated further at $37^{\circ} \mathrm{C}$. At various time points $(\mathrm{T}=6,12,24,8,72 \mathrm{hpi}$; 1wpi) supernatant was removed and infectious virus was determined using a plaque assay on BHK cells. 
Table 2-1. Cells

\begin{tabular}{|c|c|c|}
\hline Cell Type & Obtained from & Culture Medium \\
\hline BHK21 cells & Lab of Dr. Michael Whitt & $\mathrm{DMEM}^{\mathrm{a}}+5 \%$ FBS (D5) \\
\hline Mouse Embryonic & ATCC (Manassas, VA) & DMEM + 10\% FBS (D10) \\
\hline \multicolumn{3}{|l|}{ Fibroblasts (MEF) } \\
\hline L929 mouse fibroblasts & $\begin{array}{l}\text { Courtesy of Dr. Lawrence } \\
\text { Pfeffer (Pathology, } \\
\text { UTHSC) }\end{array}$ & $\mathrm{DMEM}+10 \% \mathrm{FBS}$ \\
\hline Rat Fibroblasts (FR) & ATCC (Manassas, VA) & $\mathrm{DMEM}+10 \%$ FBS $(\mathrm{E} 10)$ \\
\hline $\begin{array}{l}\text { Primary Rat Astrocytes } \\
\text { (PRAs) }\end{array}$ & Special Protocol ${ }^{\mathrm{b}}$ & $\mathrm{DMEM}+10 \% \mathrm{FBS}$ \\
\hline F98 (GFP) ${ }^{\mathrm{c}}$ Rat Glioma & ATCC (Manassas, VA) & $\mathrm{DMEM}+10 \% \mathrm{FBS}$ \\
\hline C6 (GFP) Rat Glioma & ATCC (Manassas, VA) & DMEM + 10\% FBS \\
\hline U87 (GFP) human glioma & $\begin{array}{l}\text { Courtesy of the Lab of } \\
\text { Neurosurgery (Christopher } \\
\text { Duntsch, MD, PhD; } \\
\text { UTHSC) }\end{array}$ & $\mathrm{EMEM}^{\mathrm{d}}+10 \% \mathrm{FBS}$ \\
\hline T98G human glioma & $\begin{array}{l}\text { Courtesy of the Lab of } \\
\text { Neurosurgery (Christopher } \\
\text { Duntsch, MD, PhD; } \\
\text { UTHSC) }\end{array}$ & $\mathrm{EMEM}+10 \% \mathrm{FBS}$ \\
\hline A172 human glioma & $\begin{array}{l}\text { Courtesy of the Lab of } \\
\text { Neurosurgery (Christopher } \\
\text { Duntsch, MD, PhD; } \\
\text { UTHSC) }\end{array}$ & $\mathrm{EMEM}+10 \% \mathrm{FBS}$ \\
\hline
\end{tabular}

aDulbecco's Minimum Essential Medium supplemented with 5\% Fetal Bovine Serum ${ }^{\mathrm{b}}$ Primary rat astrocyte cultures were obtained by dissecting and removing striata from E17 rat embryos. Cells were dissociated, plated, and allowed to grow to confluency for approximately 10 days.

${ }^{c}$ Cells were purchased and later transduced to express GFP. Protocol described separately

'Eagle's Minimum Essential medium 
Table 2-2. Virus constructs

\begin{tabular}{|c|c|c|}
\hline Virus & Serotype (Strain) & Constructed by \\
\hline wtVSV-GFP & $\begin{array}{l}\text { Indiana (N, P, M, G } \\
\text { genes- San Juan; L gene- } \\
\text { Mudd Summers) }\end{array}$ & Michael A. Whitt, PhD \\
\hline rNCP12.1 $1_{\text {I-GFP }}$ & $\begin{array}{l}\text { Indiana (N, P, M, G } \\
\text { genes- San Juan; L gene- } \\
\text { Mudd Summers) }\end{array}$ & Himangi Jayakar, PhD \\
\hline $\mathrm{rNCP} 12.1_{\mathrm{NJ}}-\mathrm{GFP}$ & $\begin{array}{l}\text { New Jersey (N, P, M, G } \\
\text { genes- San Juan; L gene- } \\
\text { Mudd Summers, G gene- } \\
\text { Ogden) }\end{array}$ & Himangi Jayakar, $\mathrm{PhD}$ \\
\hline rNCP12.1 1 -dsRed & $\begin{array}{l}\text { Indiana (N, P, M, G } \\
\text { genes- San Juan; L gene- } \\
\text { Mudd Summers) }\end{array}$ & Himangi Jayakar, PhD \\
\hline
\end{tabular}




\section{Cytotoxicity Assay}

Rat glioma cells and PRAs were seeded into 96 well plates then mock infected with serum free media or infected with either wt-VSV or rNCP12.1 at an MOI of 10 at $31^{\circ} \mathrm{C}$ for 1 hour. Inoculum was removed and replaced with D10 and cells were incubated at $37^{\circ} \mathrm{C}$. At various times post infection $(\mathrm{T}=6,12,24,8,72 \mathrm{hpi} ; 1 \mathrm{wpi})$, supernatants were harvested and used to determine growth kinetics as described. Cell viability at the same time points was determined using the CellTiter 96Aqueous One Solution Cell Proliferation Assay (Promega) according to the manufacturer's protocol.

\section{IFN Protection Assay}

Rat glioma cells and PRAs were plated in 96 well plates and 24 well plates respectively. Cells were counted after plating using a hemocytometer to confirm a normalized cell count. Culture media was removed from cells and rat IFN $\beta$ (rIFN $\beta$; provided by Dr. Lawrence Pfeffer, Department of Pathology, UTHSC) was added starting with $1000 \mathrm{U}$ (high dose experiment) and 100U (low dose experiment) of IFN at 2 fold serial dilutions. Cells were incubated overnight at $37^{\circ} \mathrm{C}$. At 24 hours post-treatment, IFN was removed and cells were infected with wtVSV at an MOI of 3 for $1-2$ hours at $31^{\circ} \mathrm{C}$. Inoculum was then removed, fresh D5 was added, and cells were incubated at $37^{\circ} \mathrm{C}$ overnight. At 24hpi, D5 was removed and cells were washed three times for 5 minutes each with 1 XPBS. Cells were then fixed with $3 \%$ paraformaldehyde for 20 minutes then treated with $1 \%$ TritonX-100 at room temperature for 5 minutes to permeabilize the cell membrane. The cells were stained with an $\alpha$-VSV N specific monoclonal antibody (10G4) conjugated to Rhodamine. Cell rounding and presence of virus was determined by fluorescence microscopy (Zeiss, AxioVision). Protection was defined as the amount (U) of IFN at which less than $50 \%$ cells were infected.

\section{pI:C Induction of IFN}

Cells were plated in $60 \mathrm{~mm}$ plates and cultured until $70 \%$ confluent. Cells were treated for 4 hours with 25, 50, or $100 \mu \mathrm{g} \mathrm{pI}$ :C in SF-DMEM or transfected with $0.1,1$, or $10 \mu \mathrm{g} \mathrm{pI}: \mathrm{C}$ in a ratio of 1:1 with Lipofectamine 2000. pI:C treatment mixture was removed, D10 was added, and cells were incubated at $37^{\circ} \mathrm{C}$. Supernatants were collected at 24 hours post-treatment and IFN activity was determined using the IFN bioassay described. Cell lysates were harvested and stored at $-20^{\circ} \mathrm{C}$ or used directly for qRT-PCR studies. In a subsequent time course experiment, cells plated in $60 \mathrm{~mm}$ plates were treated with $10 \mu \mathrm{g}$ of transfected $\mathrm{pI}: \mathrm{C}$ only, and supernatants were collected at $\mathrm{t}=2.5,4,6,12,18$, 24 hours post-transfection. Supernatants were used to determine presence of active IFN using the IFN bioassay and remaining cells were lysed with $1 \mathrm{ml}$ Trizol reagent (Invitrogen), harvested, and stored at $-20^{\circ} \mathrm{C}$ or used directly in qRT-PCR studies. 


\section{IFN Bioassay}

Supernatants from immune-stimulated cells (pI:C pretreatment or viral infection) were collected at various time points following treatment and applied at 2 fold serial dilutions on L929 mouse fibroblasts pre-plated in 96 well plates to reach $70 \%$ confluency at the time of the experiment. After a 24 hour incubation, the supernatant was removed and L929 cells were infected with wtVSV at an MOI of 0.5 for $1 \mathrm{hr}$ at $31^{\circ} \mathrm{C}$. Inoculum was removed, fresh media was added, and cells were incubated at $37^{\circ} \mathrm{C}$. Rat IFN $\beta$ (rIFN $\beta$ ) was used as the standard for scoring of the IFN bioassay. Units for rIFN $\beta$ were determined with respect to the international reference standard for mouse interferon alpha/beta, mouse IFN- $\alpha / \beta(\mathrm{NIH})$ and stored in aliquots having a known titer of $10^{6} \mathrm{U} / \mathrm{mL}$. At 36-48hpi, media was removed and the assay was scored as determined by the dilution factor at which 50\% inhibition of CPE was observed [367]. This dilution was then compared to the known concentration at which the standard inhibited 50\% CPE. Based on the data in Table 2-3, the highest dilution at which rIFN standard inhibited CPE is $1.5625 \mathrm{U} / 125 \mu \mathrm{L}=0.0125 \mathrm{U} / \mu \mathrm{L}=12.5 \mathrm{U} / \mathrm{mL}$. From this information, we can determine the original amount of IFN in each sample prior to dilution (Example: CPE in sample \#5 started at $25 \mathrm{U} / 125 \mu \mathrm{L}$ therefore titer of the original sample would be $200 \mathrm{U} / \mathrm{mL}$. Sample 2 showed no CPE therefore an additional study evaluating higher dilutions would be necessary. Sample \#4 showed no protection, therefore IFN titer of this sample is undetectable).

\section{Viral Infection for IFN Bioassay and ISG qRT-PCR}

Cells were plated in $60 \mathrm{~mm}$ plates and grown to $80 \%$ confluency. Cells were then mock-infected (SF media) or infected with either wt-VSV or rNCP12.1 at an MOI of 10, for 1 hour at $31^{\circ} \mathrm{C}$. Inoculum was removed and cells were washed twice with SFDMEM, then incubated overnight at $37^{\circ} \mathrm{C}$ in $2 \mathrm{~mL}$ of D-10. At time points, $\mathrm{T}=6,12,18$, and 24hpi supernatants were harvested and centrifuged to remove cellular debris. Remaining cells were lysed in $1 \mathrm{ml}$ Trizol reagent (Invitrogen), harvested, and stored at $20^{\circ} \mathrm{C}$ or used directly in qRT-PCR studies. In order to be used for IFN bioassays, virus was separated from supernatant of infected cells using Millipore Amicon Ultra-4 centrifugal filters with Ultracel membrane of 100,000 NMWL cutoff. Samples were centrifuged twice at 5000rpm for 10 minutes at $4^{\circ} \mathrm{C}$, using a new filter for the second spin cycle. Standardization of this IFN bioassay using virus-containing samples will be explained in Appendix B. A small aliquot of ultra-filtered supernatant was used to ensure all virus was removed using a standard plaque assay on BHK21 cells.

\section{qPCR to Determine Expression of IFN $\beta 1$}

Semi-quantitative RTPCR was carried out to detect expression of IFN $\beta 1$ in virally treated FR and C6 rat glioma cells at 6 and 12hpi. Infected cells were lysed in $1 \mathrm{~mL}$ Trizol and total RNA was extracted. A One step RT-PCR (Qiagen) was carried out according to manufacturer protocol using IFN $\beta 1$ specific primers (Table 2-4). DNA 
Table 2-3. Scoring for IFN bioassay

\begin{tabular}{lcccccccc}
\hline & \multicolumn{7}{c}{ Initial Concentration $(U / 125 \mu L) \rightarrow$ Final Concentration $(U / m L)$} \\
\cline { 2 - 9 } Sample & $\mathbf{1 0 0 \rightarrow}$ & $\mathbf{5 0 \rightarrow}$ & $\mathbf{2 5} \rightarrow$ & $\mathbf{1 2 . 5 \rightarrow}$ & $\mathbf{6 . 2 5} \rightarrow$ & $\mathbf{3 . 1 2 5} \rightarrow$ & $\mathbf{1 . 5 6 2 5} \rightarrow$ & $\mathbf{0 . 7 8 1 2 5 \rightarrow}$ \\
& $\mathbf{8 0 0}$ & $\mathbf{4 0 0}$ & $\mathbf{2 0 0}$ & $\mathbf{1 0 0}$ & $\mathbf{5 0}$ & $\mathbf{2 5}$ & $\mathbf{1 2 . 5}$ & $\mathbf{6 . 2 5}$ \\
\hline IFN std & - & - & - & - & - & - & $-/+$ & + \\
SF only & + & + & + & + & + & + & + & + \\
Sample 1 & - & - & - & - & $-/+$ & + & + & + \\
Sample 2 & - & - & - & - & - & - & - & - \\
Sample 4 & + & + & + & + & + & + & + & + \\
Sample 5 & - & - & $-/+$ & + & + & + & + & + \\
Sample 6 & - & $-/+$ & + & + & + & + & + & + \\
\hline
\end{tabular}


Table 2-4. qRT-PCR UPL primers for interferon stimulated genes

\begin{tabular}{|c|c|c|c|}
\hline Gene & Left Primer & Right Primer & $\begin{array}{c}\text { UPL } \\
\text { Probe \# }\end{array}$ \\
\hline $\begin{array}{l}\text { IFN } \beta 1 \\
\text { (interferon beta } 1 \\
\text { fibroblast) }\end{array}$ & ggtggaccctccacattg & tagtcgatggagagggcagt & 18 \\
\hline $\begin{array}{l}\text { IFNa4 } \\
\text { (interferon } \\
\text { alpha 4) }\end{array}$ & cagcagctcagtgacctcaa & taggggaggttcttgcattc & 62 \\
\hline $\begin{array}{l}\text { Mx1 (myxovirus/ } \\
\text { influenza virus } \\
\text { resistance 1) }\end{array}$ & ccagcacctgaatgcctac & tggagtactggatgatcagagg & 94 \\
\hline $\begin{array}{l}\text { GBP1 } \\
\text { (guanylate } \\
\text { binding } \\
\text { protein 1) }\end{array}$ & cagaaaaggaaaaggagattgaag & ttctgtgtttcctccaacagc & 66 \\
\hline $\begin{array}{l}\text { Plscr1 } \\
\text { (phospholipid } \\
\text { scramblase 1) }\end{array}$ & tcagattctggttcatcagcag & cgtatcttccaccgcaaagt & 113 \\
\hline $\begin{array}{l}\text { RIG-I } \\
\text { (Ddx } 58 \text {, DEAD } \\
\text { box polypeptide } \\
58 \text { ) }\end{array}$ & gaagattctggaccccacct & tgaatgcactgcacctcatc & 73 \\
\hline $\begin{array}{l}\text { IRF1 } \\
\text { (interferon } \\
\text { regulatory } \\
\text { factor 1) }\end{array}$ & aagggaagttacctgaggacatc & gctgaagtctccatagacagtagagag & 92 \\
\hline Cyclophilin B & acgtggttttcggcaaagt & cttggtgttctccaccttcc & 97 \\
\hline
\end{tabular}


fragments were separated by $2 \%$ agarose gel and visualized under UV light following treatment with ethidium bromide.

\section{Quantitative RT-PCR (qRT-PCR) to Determine Expression of IFN $\beta 1$ and ISGs}

After Trizol extraction of total RNA, RNA concentration in each sample was determined by spectrophotometer at $\mathrm{A}_{260}$. One microgram of RNA per reaction, along with M-MLV reverse transcriptase (Invitrogen) and random hexamers were used to synthesize cDNA. Next, quantitative PCR was performed on the LightCycler 480 (Molecular Resource Center, UTHSC) using Universal Primary Library (UPL) protocol for rat gene expression (Roche) (Table 2-4). Universal probes are specific for the detection of the rat transcriptome and are labeled with fluorescein at the 5 ' end and a dark quencher dye at the 3 'end that allow detection by standard SYBR Green I filters. The LightCycler 480 Taqman90 protocol was used which is pre-programmed to perform the following cycles: activation of DNA Polymerase for 5 minutes at $95^{\circ} \mathrm{C}$; amplification of cDNA for 40 cycles which includes 10 seconds at $95^{\circ} \mathrm{C}$ denature, 20 seconds at $60^{\circ} \mathrm{C}$ annealing, and 10 seconds at $72^{\circ} \mathrm{C}$ extension; cool down for 30 seconds at $40^{\circ} \mathrm{C}$.

\section{Western Blot Detection of Neutralizing Antibodies}

To detect the presence of $\alpha$-VSV antibodies in the sera of treated animals, blood was collected at the time of sacrifice by transcardiac method (described below). The cellular components were separated from blood by centrifugation and serum only was stored at $-20^{\circ} \mathrm{C}$ until used. $10^{4}$ pfu rNCP12.1 were prepared for electrophoresis in reducing sample buffer LSB +BME. Proteins were separated using 9\% acrylamide 10\% SDS gel electrophoresis then transferred to polyvinylidene fluoride (PVDF) microporous membrane. Membranes were blocked using 5\% Non-fat dry milk/TTBS for two hours and viral proteins probed with rat sera from treated or control animals diluted to 1:100 or with positive control, R6-F, at 1:5000 (R6-F is a rabbit polyclonal antibody directed against detergent treated wtVSV). Blots were incubated for two hours at room temperature or overnight at $4^{\circ} \mathrm{C}$ followed by treatment with goat $\alpha$-rat secondary antibody conjugated to Horse Radish Peroxidase (HRP) (Jackson ImmunoResearch Laboratories) at a dilution of 1:5000 for two hours at room temperature. Signal was visualized using chemiluminescence (SuperSignal West Dura, Pierce) followed by exposure to and development of X-ray film.

\section{Neutralizing Antibody Assay}

In 96 well plate, BHKs were plated and cultured overnight in D5. Serum samples were treated at $56^{\circ} \mathrm{C}$ for 35 minutes in order to neutralize any residual virus. $200 \mathrm{pfu}$ of wtVSV were added to two fold serial dilutions (1:2 to 1:1000) of test sera. Negative control included FBS only and positive control included TN-1, a polyclonal antibody developed in rabbit against i.v. injection of UV-inactivated intact wtVSV. Serum or 
$\mathrm{TN}-1$ plus virus was incubated at $37^{\circ} \mathrm{C}$ for $35-45$ minutes then added to cells overnight at $37^{\circ} \mathrm{C}$. Neutralizing antibody titer was determined by the inverse of the dilution required for $50 \%$ inhibition of infection at 24 hours post-treatment (e.g. 1:1000 dilution corresponds with a neutralizing titer of 1000).

\section{Animal Studies}

\section{Tumor Implantation}

All animal experiments were conducted under the guidelines of the UTHSC IACUC (Institutional Animal Care and Use Committee). Both Wistar and Fischer 344 rats were obtained from Charles River. To establish intracranial gliomas, six week old 250-300 gram male adult rats were anesthetized by administration of ketamine/xylazine i.p. at a dosage of $87 \mathrm{mg} / 13 \mathrm{~kg}$ body weight $(0.1 \mathrm{~mL} / 100 \mathrm{~g}$ body weight). Animal was placed in a stereotaxic frame and skin was prepped using iodine. By sterile technique, a $1.0 \mathrm{~cm}$ incision was made in the midline of the skull along the sagittal suture starting just behind the plane connecting the eyes. The cranium was exposed and a Burr hole $3.0 \mathrm{~mm}$ lateral to the bregma on the right of the bregmatic suture was created without compromising the dura. 1 × $10^{5}$ rat glioma cells (C6-GFP; F98-GFP) were injected intracranially using a $25 \mu \mathrm{L}$ Hamilton Syringe. Injections were given slowly over 30 seconds at a depth of $3 \mathrm{~mm}$ below the dura. Following administration of cells, the needle was left in place for approximately 2 minutes then retracted slowly over 30 seconds. Following implantation, the Burr hole was sealed with bone wax and the skin incision was sutured using 4.0 monofilament. Throughout the procedure, animals were kept on a warming blanket in order to maintain body temperature. Post-op, animals were treated with oxygen to aid in recovery from anesthesia.

\section{Measures of Morbidity}

Morbidity was determined based on weight trends and the rat coma scale (RCS) (Table 2-5). The RCS was developed by Christopher Duntsch, MD, PhD (UTHSC Department of Neurosurgery) as an adaptation of the human Glasgow coma scale (GCS), a widely used assessment tool for neurological function especially following traumatic brain injury. Components of the RCS involve level of consciousness, motor function, and spontaneous eye movement. Additional manifestations of distress are not accounted for in the RCS therefore we created a supplemental assessment to help determine the need for euthanasia which include rat-specific signs and symptoms such as chattering and lack of grooming (Table 2-6). Certain signs are more severe in nature and would call for euthanasia when they are present as animals are less likely to recover from these. 
Table 2-5. Rat coma scale

\begin{tabular}{|c|c|c|c|c|}
\hline \multirow{2}{*}{$\begin{array}{c}\text { Neurological } \\
\text { Response }\end{array}$} & \multicolumn{4}{|c|}{ Score* } \\
\hline & 4 & 3 & 2 & 1 \\
\hline $\begin{array}{l}\text { Level of } \\
\text { Consciousness } \\
\text { (LOC) }\end{array}$ & $\begin{array}{l}\text { Moves } \\
\text { spontaneously } \\
\text { without } \\
\text { stimulation }\end{array}$ & $\begin{array}{l}\text { Moves } \\
\text { spontaneously } \\
\text { when picked up } \\
\text { for } 3 \text { seconds } \\
\text { by tail and put } \\
\text { back down }\end{array}$ & $\begin{array}{l}\text { Moves with } \\
\text { pain stimulus }\end{array}$ & $\begin{array}{l}\text { Does not } \\
\text { move with } \\
\text { pain stimulus }\end{array}$ \\
\hline Motor & $\mathrm{N} / \mathrm{A}$ & $\begin{array}{l}\text { No motor } \\
\text { deficits }\end{array}$ & $\begin{array}{l}\text { Paresis (hemi/ } \\
\text { quadri/ } \\
\text { forelimb/ } \\
\text { hindlimb } \\
\text { involvement) }\end{array}$ & $\begin{array}{l}\text { Plegia (hemi/ } \\
\text { quadri/ } \\
\text { forelimb/ } \\
\text { hindlimb } \\
\text { involvement) }\end{array}$ \\
\hline Eyes & $\begin{array}{l}\text { Opens } \\
\text { spontaneously }\end{array}$ & $\begin{array}{l}\text { Opens with } \\
\text { stimulation }\end{array}$ & $\begin{array}{l}\text { Open to pain } \\
\text { stimulus }\end{array}$ & Does not open \\
\hline
\end{tabular}

*Highest total score, $11(4+3+4)$; Lowest total score, $3(1+1+1)$ 
Table 2-6. Additional signs of distress

\begin{tabular}{lcc}
\hline \multicolumn{1}{c}{ Sign } & Severity & Predicted Outcome \\
\hline $\begin{array}{l}\text { Sneezing/ coughing } \\
\text { Bowel changes (usually } \\
\text { diarrhea) }\end{array}$ & Low & Usually recover \\
$\begin{array}{l}\text { Porphyrin stain around } \\
\text { eyes }\end{array}$ & Low & \\
$\begin{array}{l}\text { Ruffled hair } \\
\text { Huddles in corner }\end{array}$ & Medium & Monitor weight and RCS \\
$\begin{array}{l}\text { Hunched } \\
\text { Chattering/ vocalizing }\end{array}$ & Medium & every day versus every 3 \\
Urine stained coat & Medium \\
$\begin{array}{l}\text { Late seizure activity } \\
\text { Increased work of } \\
\text { breathing }\end{array}$ & Medium & Pending death \\
\hline
\end{tabular}




\section{Cerebrospinal Fluid (CSF) Collection}

The procedure for CSF collection was adapted from the protocol described in Sharma et al, 2010 [368] and performed as a terminal procedure. Immediately following induction of deep anesthesia, animal was placed in position on the CSF collection apparatus provided by the UTHSC Department of Comparative Medicine, Laboratory Animal Care Unit, in order to maintain proper head positioning and easier identification of access to the cisterna magna, located between the occipital protuberance and the posterior tubercle of the atlas. Once secured, hair covering the occipital region was shaved. A winged 25 gauge $x$ 3/4 inch needle (BD Vacutainer Safe Lok) with infusion tubing was connected to a $1 \mathrm{cc}$ syringe. The needle was slowly inserted into the site of the cisterna magna directly below the occipital protuberance at an angle as close to parallel as possible to the position of the spine. Once dura mater was penetrated as detected by a subtle decrease in resistance, gentle negative pressure was applied by slow suction using the 1cc syringe. Approximately $50-70 \mu \mathrm{L}$ of CSF can be obtained by this method.

\section{Transcardiac Blood Collection}

Collection of blood by transcardiac method was performed as a terminal procedure following induction of deep anesthesia. A 1-2 cm incision was made on the skin of the abdomen and widened with scissors to make a skin flap for access to the intraabdominal cavity. After entering the abdomen, the xiphoid process was elevated and the diaphragm was appreciated. The diaphragm was pierced with the scissors and the incision was widened in order to enter the thoracic cavity inferiorly. Once inside the thoracic cavity, the left ventricle of the heart was identified and $0.2 \mathrm{cc}$ heparin was injected to prevent coagulation during blood collection. After $\sim 30-60$ seconds, a 27 gauge $\mathrm{x} 1 / 2$ needle attached to a $3 \mathrm{cc}$ syringe was inserted into the left ventricle and blood was collected and stored immediately on dry ice to be used for subsequent studies.

\section{Preparation of Concentrated Virus for Intracranial Injections}

Forty $150 \mathrm{~mm}$ plates of BHKs were grown to $80-90 \%$ confluency. Cells were infected at an MOI of 3 in 10mL of SF-DME (no penicillin or streptomycin, -P/S) for 1 hour at $31^{\circ} \mathrm{C}$. After one hour, $10 \mathrm{~mL}$ additional SF-DME -P/S was added to cells and cells were incubated at $37^{\circ} \mathrm{C}$ overnight. Supernatants from infected cells were harvested and cellular debris was removed by centrifugation (2550rpm for 10 minutes at $4^{\circ} \mathrm{C}$ using JS 4.2 rotor). A small aliquot of supernatant was collected and stored at $-20^{\circ} \mathrm{C}$ for later titering. Next supernatant was passed over a $20 \%$ sucrose cushion (TN pH 7.2) and virus was pelleted at $28,000 \mathrm{rpm}$ for 60 minutes at $4^{\circ} \mathrm{C}$. Viral pellet was resuspended in $100 \mu \mathrm{L}$ sterile TN pH $7.2+10 \%$ sucrose. A small aliquot of pelleted virus was tittered using a standard plaque assay on BHKs and viral yield was compared to titers of pre-pellet aliquot to determine efficiency of recovery. 


\title{
rNCP12.1 Single Injection
}

Three to five days post-tumor implantation, rats were divided randomly into groups and viral injection was administered using the same injection procedure as performed for tumor implantation. Through the pre-existing Burr hole, a single dose of rNCP12.1-GFP at either $10^{5}, 10^{6}, 10^{7}$, or $10^{8} \mathrm{pfu} / 25 \mu \mathrm{L}$ Tris-Saline $+10 \%$ sucrose $(\mathrm{TN}-$ 10) buffer was administered by Hamilton syringe. Vehicle only (TN-10) was given to control animals. Animals were monitored daily for changes in weight and for signs of illness or treatment-related neurological deficits. On day 15 post-tumor implantation, animals were sacrificed and the brains were harvested for further tissue processing. A second group of treated and control animals were followed until RCS score, weights, and/or other clinical signs called for euthanasia.

\begin{abstract}
Alzet Pump
On day five post- tumor implantation, ALZET pumps were prepared and filled with viral agent or viral vehicle in a sterile field according to manufacturer's instructions. Pumps were placed in $0.9 \%$ sterile saline and incubated for $4-6$ hours at $37^{\circ} \mathrm{C}$ as priming for the delivery of agent at the time of implantation. Animals were anesthetized and placed onto the stereotaxic frame. The previous incision site created during tumor implantation was opened and slightly widened to allow enough space for the pump implant. Injection of rNCP12.1-GFP was administered as described. Following injection, pre-filled ALZET pumps were implanted taking care to not damage the catheter and needle. The needle was inserted directly into the pre-made Burr hole. Once in place, the overlying skin was sutured with the pump in order to secure it into place. The incision site was again sutured with 4.0 monofilament. Animals were followed until RCS score, weights, and/or other clinical signs called for euthanasia.
\end{abstract}

\section{Serotype Switch}

On day 5, post-tumor implantation, rats were divided randomly into groups and viral injection was administered as described. A single dose of rNCP12.1-GFP, Indiana serotype was administered at a dose of $10^{9} \mathrm{pfu} / 10 \mu \mathrm{L}$ TN buffer by Hamilton syringe. Control animals received TN buffer vehicle only. On day 10, post-tumor implantation, a second dose of $10^{9} \mathrm{pfu} / 10 \mu \mathrm{L}$ rNCP12.1-GFP was administered. Half of treated animals received Indiana serotype as the second injection and the other half received New Jersey serotype. On day 15 post-tumor implantation, animals were sacrificed and the brains were harvested for further tissue processing. A second group of treated and control animals were followed until RCS score, weights, and/or other clinical signs called for euthanasia. 


\section{Test for Tumorigenicity of Pre-Infected Tumor Cells}

Three $10 \mathrm{~cm}$ dishes of F98-GFP cells were plated and grown overnight. Cells were infected at an MOI of 0.1 or 10 with rNCP12.1-GFP for one hour at $31^{\circ} \mathrm{C}$. Cells were washed three times with SF-DME, trypsinized, centrifuged and resuspended in $5 \mathrm{~mL}$ of PBS. Uninfected cells were also trypsinized, centrifuged and resuspended in $5 \mathrm{~mL}$ of PBS. Cells were counted and aliquots of infected (MOI of 0.1 and 10) to uninfected cells at a ratio of $1: 10$ or 1:1 were made based on cell counts using a hemocytometer. A total of 100,000 cells $/ 10 \mu 1$ were prepared and implanted by the standard protocol.

\section{Pre-Infected Tumor Cell Carriers in Tumor Bearing Rats}

F98-GFP glioma cells were plated in $10 \mathrm{~cm}$ dishes and grown overnight. Cells were infected at MOI of 3 for one hour at $31^{\circ} \mathrm{C}$. Cells were washed three times with SFDME, trypsinized, centrifuged, and resuspended in 5mL PBS. Cells were diluted to 1000 cells/ $10 \mu \mathrm{L}$ and implanted per usual protocol.

\section{Recovery of Virus from PC-Treated Animals}

Sera, CSF, and non-fixed brain tissue from several animals was harvested. A $0.5 \mathrm{~cm}^{3}$ area starting at the needle track continuing rostrally was excised from brain tissue and placed in $2 \mathrm{~mL}$ PBS in a $15 \mathrm{~mL}$ conical centrifuge tube. Tissue was homogenized for 2-3 minutes and cellular debris was removed by centrifugation. One hundred microliters of each sample was added to cultured BHK cells for 1 hour at $31^{\circ} \mathrm{C}$. Samples were removed, warm agar containing D5 was applied, and cells were incubated at $37^{\circ} \mathrm{C}$. At 24-36 hpi, agar was removed and cells were fixed and stained to detect VSV nucleocapsid using an $\alpha \mathrm{VSV}-\mathrm{N}$ mAb (10G4) conjugated to Rhodamine. Fluorescence images were captured and processed using Zeiss Axiovision.

\section{Tissue Processing}

Brains were harvested at the time of sacrifice following vascular perfusion and fixation. For all experiments, perfusion included a vascular flush of $200 \mathrm{~mL} 0.9 \% \mathrm{NaCl}$ followed by fixation with $200 \mathrm{~mL} 4 \%$ paraformaldehyde. For brain tissue harvested from animals treated with rNCP12.1-GFP pre-infected tumor cells, only a vascular flush of $400 \mathrm{~mL} 0.9 \%$ saline was done without fixative as tissue was needed for qRT-PCR analysis. Following fixation, brains were soaked in 4\% paraformaldehyde (except for brain tissue harvested for qRT-PCR) for 24 hours and then $20 \%$ sucrose for 2 days. Cryosectioning of brain tissue was done starting at the site of injection (needle track) in $10 \mu \mathrm{m}$ thick sections for samples used for Hematoxylin (Gill's) and Eosin Y (Fisher Scientific, Suwanee, GA) (H\&E) staining and at $14 \mu \mathrm{m}$ thick sections for immunohistochemistry (IHC). The site of sectioning at and adjacent to the needle track was chosen as we assume this is the most likely site for tumor implantation although 
variations do occur. For IHC staining of tissue, sections were treated with $2 \%$ NFDM (nonfat dry milk) in $0.2 \mathrm{M}$ PBS/0.3\% triton X-100 to block nonspecific staining. Tissue was washed three times for 15 minutes each in PBS at room temperature. The primary antibodies, rabbit $-\alpha$ GFAP at 1:80 and mouse- $\alpha$ VSV N protein (10G4) at 1:3, were used to probe for the astrocytic marker, GFAP, and VSV Nucleocapsid (N) protein, respectively. Signal was amplified using the secondary antibodies, goat- $\alpha$ rabbit conjugated to Cy5 (blue) and goat - $\alpha$ mouse conjugated to Cy3 (red) (Jackson ImmunoResearch Laboratories) both at a concentration of 1:100 for 1-2 hours at room temperature. All antibodies were diluted in $0.1 \%$ Triton-X /PBS containing the serum of the species from which antibody was derived. Lastly, slides were washed and coverslipped. Fluorescent markers were visualized using laser scanning confocal microscopy (Zeiss LSM 510 AIM version 3.2 software). Slices were captured at magnifications of $0.4 \mathrm{X}, 10 \mathrm{X}, 20 \mathrm{X}$, and $40 \mathrm{X}$.

\section{Tumor Measurement}

Tumor surface area was determined based on images collected from fluorescence microscopy (Leica). Using NIH Image J software, an outline of GFP- expressing tumors was traced and surface area was determined using a pre-set calibrated scale in millimeters (Set Scale command). For rNCP12.1 pre-infected tumor cell experiment only, ImageScope (Aperio ePathology Solutions) software was used to measure and calculate tumor load.

\section{Statistical Analysis}

All data were analyzed using Prism Graphpad 6.0 software and expressed as mean \pm standard deviation. A One-Way ANOVA with multiple comparisons to an untreated control group was done for experiments having 3 or more groups while a two tailed t-test was used for comparison of the means for experiments having no more than two groups. For the evaluation of rNCP12.1-GFP pre-infected cells, multiple t-tests were done comparing treated versus untreated groups at each time point. The Kaplan-Meier analysis of survival for animal experiments was done using a log-rank (Mantel-Cox) test. $\mathrm{P}$ values of $<0.05$ were considered to be statistically significant. 


\section{CHAPTER 3. RESULTS}

\section{In Vitro Characterization of rNCP12.1}

\section{rNCP12.1 Retains Its Non-Cytopathic Phenotype in Normal Brain Cells While Remaining Cytopathic in Most Tumor Cell Lines}

As discussed, rNCP12.1 is a recombinant VSV vector harboring mutations that prevent its ability to form the truncated forms of the matrix protein. This promotes its non-cytopathic phenotype in a number of cell lines including BHK21 cells. In evaluating rNCP12.1 as a potential oncolytic virus for the treatment of malignant brain tumors, we wanted to first establish the cell rounding phenotype in cells of glial origin as this is associated with viral-induced cytopathic effects. In order to do so, a cell rounding assay was performed in primary rat astrocytes (PRAs) and in two rat glioma cell lines, F98GFP and C6-GFP. Cells were infected with both wtVSV and rNCP12.1 at an MOI of 10 and cell rounding was observed at 24hpi. rNC12.1- infectedPRAs demonstrated minimal cell rounding at $24 \mathrm{hpi}$ in comparison to those infected with wtVSV. This was similar to cell rounding previously seen in BHK-21 cells. On the other hand, rNCP12.1 infection in F98 cells caused rounding and cells became detached from the culture plate by $24 \mathrm{hpi}$ comparable to wtVSV infection. C6 glioma cells demonstrated a cell rounding variant in which the majority of cells were rounded but not detached from the plate (data not shown). The cell rounding phenotype of rNCP12.1 infection was also determined using an MOI of 1 versus 10 in three human glioma cell lines, U87-GFP, A172, and T98G (Figure 3-1). As with rat glioma cells, there was variation in cell rounding. T98G glioma cells were least sensitive to rNCP12.1 infection with very few rounded cells. Though cells were not rounded, they did display a decrease in confluency at 24hpi in comparison to uninfected cells (Figure 3-1E and F). Both U87-GFP (Figure 3-1A and B) and A172 (Figure 3-1C and D) human glioma cells were highly sensitive to rNCP12.1 infection and demonstrated wildtype like cell rounding.

We next sought to determine if the observed cell rounding phenotypes with rNCP12.1 infection corresponded to differences in cell viability. An MTS cytotoxicity assay was used in order to measure viability following infection at an MOI of 10 over a time range of 6 hours to 1week (168 hours) (Figure 3-2A). In F98-GFP glioma cells, rNCP12.1 maintained close to wildtype cytotoxicity. Viability decreased at a similar rate to wildtype infection, dropping to $32 \%$ within $48 \mathrm{hpi}$. C6-GFP glioma cells were $\sim 90 \%$ viable relative to uninfected cells following infection with both viruses during the first day of infection, eventually dropping to approximately $40 \%$ by 48 hours. As expected, cytotoxicity in PRAs showed the greatest attenuation during rNCP12.1 infection, with cells maintaining 65-70\% viability at one week post-infection. In contrast, wt-VSV infection in PRAs displayed a similar cytotoxic profile to that observed in F98 glioma with viability dropping to nearly $30 \%$ by 48 hpi.

In addition to the lack of cell rounding, cell specific differences in viral yield were 

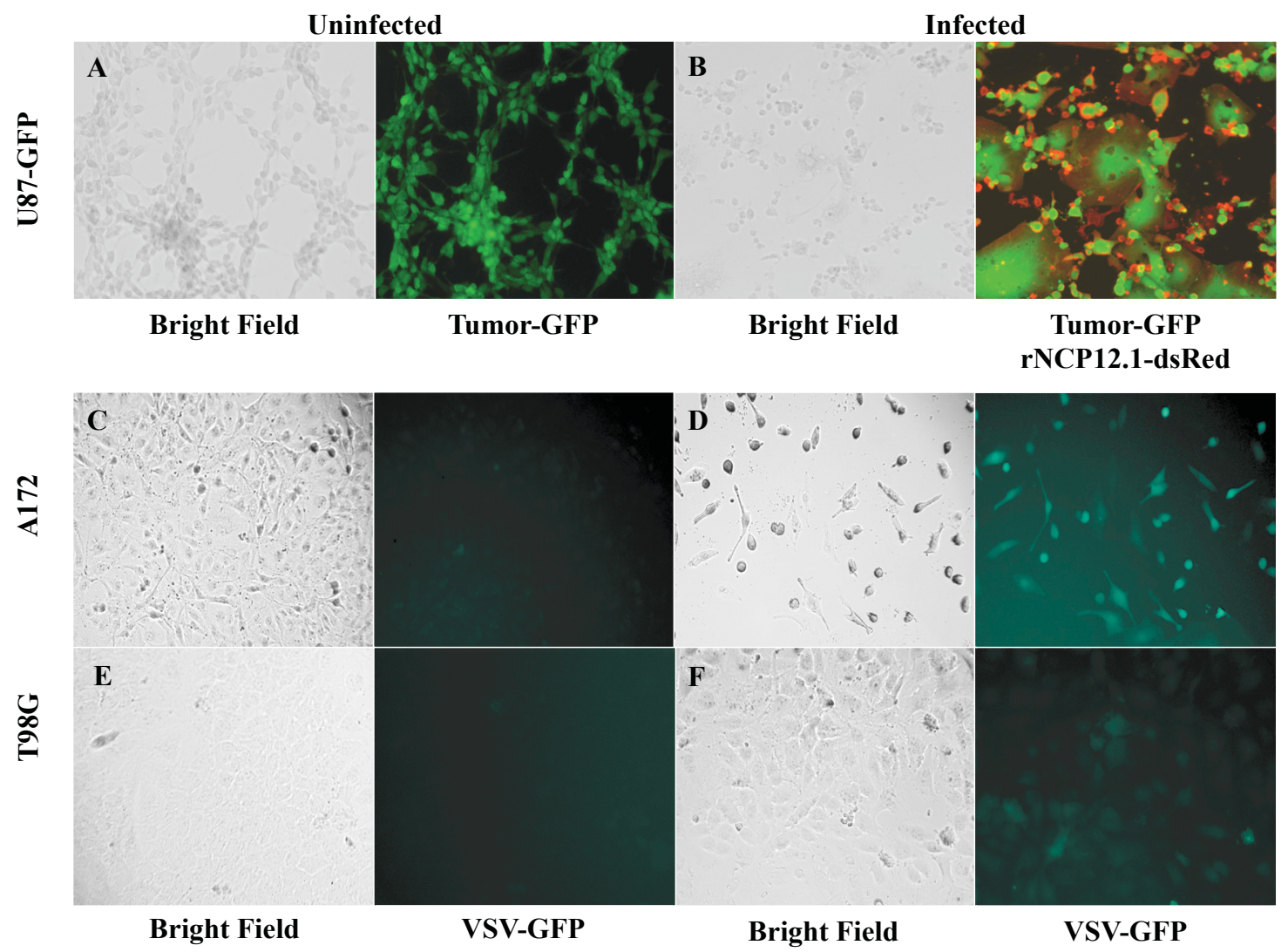

Figure 3-1. Cell rounding phenotype of rNCP12.1 in human glioma cells Human glioma cell lines, (A, B) U87-GFP (C, D) A172 (E, F) T98G were infected with either (B) rNCP12.1-dsRed or (D,F) rNCP12.1-GFP at an MOI of 10 for 1 hour at $31^{\circ} \mathrm{C}$. At $24 \mathrm{hpi}$, culture medium was removed and cells were fixed with $3 \%$ paraformaldehyde. Images were obtained by bright field and fluorescence microscopy at 10X magnification 

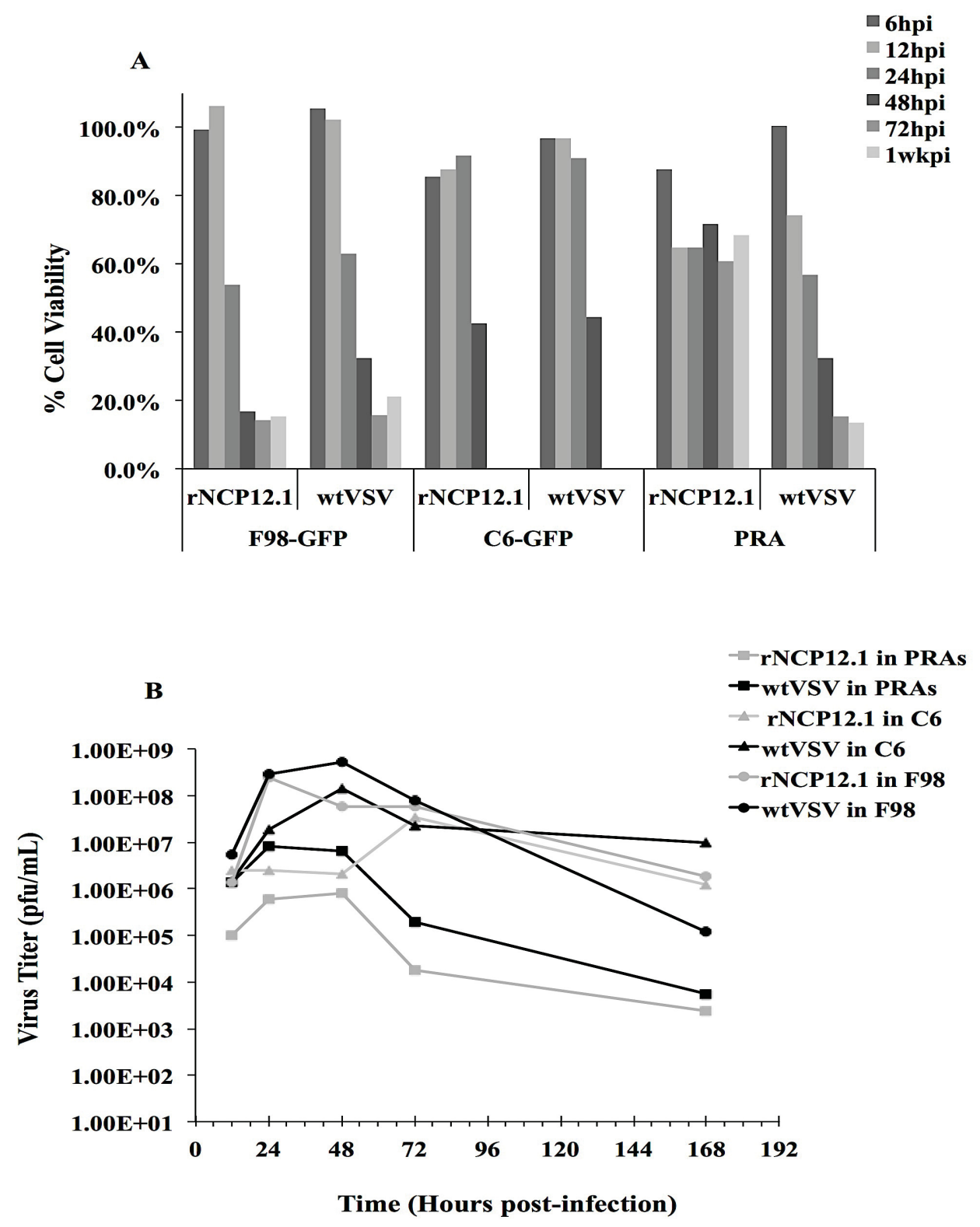

Figure 3-2. Characterization of rNCP12.1 in glioma cells

(A) MTS cell viability assay measuring cytotoxicity of F98-GFP glioma and primary rat astrocytes (PRAs) following infection with either wtVSV or rNCP12.1 at an MOI of 10. At $\mathrm{t}=6,12,24,48,72 \mathrm{hpi}$, and $1 \mathrm{wkpi}$, culture medium was removed and MTS reagents using Cell Titer Assay (Promega) were added to cells for 4 hours at $37^{\circ} \mathrm{C}$. Cell viability was calculated based on changes in absorbance at $490 \mathrm{~nm},\left(\mathrm{~A}_{490}\right)$. Values are reported as percent (\%) absorbance relative to uninfected cells at the same time point. (B) Growth Curve for rNCP12.1 versus wtVSV in PRAs and glioma cells. Astrocytes or glioma cells (F98-GFP, C6-GFP) were infected with either wtVSV or rNCP12.1-GFP at an MOI of 10. At $\mathrm{t}=12,24,48,72 \mathrm{hpi}$ and 1 wpi, supernatants were harvested and used to determine viral titer by standard plaque assay in BHK cells. [pfu, plaque forming units; hpi, hours post infection; wpi, week post infection] 
observed. To determine viral yield in glial cells, one step growth curves were obtained over the course of 1 week following infection in rat non-tumor and tumor glial cells (Figure 3-2B). Supernatants from cells infected at an MOI of 10 were collected at various time-points and titers were obtained using a standard plaque assay in BHK cells.

Previous studies have shown that rNCP12.1 viral yield in BHK cells is 10 fold lower than that of wtVSV [237]. Similar to these results, rNCP12.1 yields were 10-100 fold lower than wtVSV at several time points during infection in both tumor and nontumor cell lines. However, in glioma cells, rNCP12.1 titers eventually reached wtVSV titers by $72 \mathrm{hpi}$ whereas rNCP12.1 was consistently 10 fold lower throughout all time points in primary astrocytes. We also observed that wtVSV growth in primary astrocytes was attenuated with yields $10^{1}-10^{4}$ fold lower than titers obtained from wtVSV infection in tumor cell lines throughout the time course. Mutations in rNCP12.1 had the most effect on viral yield in primary astrocytes with titers also ranging between $10^{1}-10^{4}$ fold lower than rNCP12.1 titers in glioma cells. There were also cell specific differences in the rate of viral production. Though viral yields were different between wtVSV and rNCP12.1 in PRAs, the rate of infectious particles produced over time were congruent. Rates of viral yield in F98 cells were similar with exception of an earlier drop in yield during rNCP12.1 infection at 48hpi compared to a drop at 72hpi with wtVSV infection. On the other hand, consistent with results from the cell viability assay, C6 glioma cells lagged behind in viral yield during the first 48 hours of infection in comparison to F98. Whereas wtVSV slowly increased over 48 hours to yields similar in F98, rNCP12.1 titers remained stagnant at approximately $2 \times 10^{6} \mathrm{pfu} / \mathrm{mL}$ during this same time period, eventually increasing to wtVSV titers by $72 \mathrm{hpi}$.

These results confirm those of previous studies showing variations in VSVinduced CPE and growth based on cell type [237, 358, 366, 369]. In the glial cells tested, there was a correlation between cell rounding and cell viability, which paralleled viral growth kinetics. Growth and cytotoxicity of rNPC12.1 in PRAs were better controlled throughout infection whereas C6s revealed early but non-sustainable control that ultimately ended in cell death. F98s, however, were less effective at controlling infection and therefore were more sensitive to the cytopathic effects of rNCP12.1.

\section{rNCP12.1 Upregulates IFN $\beta$ Expression and Increases Production of Active IFN over wtVSV in a Cell Specific Manner}

It has been shown that VSV-induced cell rounding and apoptosis are genetically linked with inhibition of host cell gene expression during infection [245, 250-254, 275]. This inhibition is attributed to inhibition by the matrix protein at various stages of gene expression including transcription, nucleocytoplasmic transport, and protein translation. The expression of IFN and IFN stimulated genes are known to be greatly affected by $\mathrm{M}$ protein and VS virions harboring specific mutations in matrix have defects in the inhibition of IFN related antiviral responses [266, 289-291]. To determine if the matrix mutant, rNCP12.1, possessed the same inability to inhibit these responses following infection in both normal and tumor brain cells, we compared levels of IFN $\beta$ expression 
following infection with wtVSV and $\mathrm{rNCP} 12.1$ in the $\mathrm{C} 6$ glioma cell line compared to a rat fibroblast cell line (FR). Using semi-quantitative reverse transcriptase-polymerase chain reaction (RT-PCR), we first tested for presence of IFN $\beta$ message following infection with either wtVSV or rNCP12.1 at an MOI of 10 at 6 and $12 \mathrm{hpi}$ (Figure 3-3A). We saw that wtVSV induced very low levels of IFN $\beta$ expression at both time points in FR cells while almost negligible amounts were expressed in C6 cells. rNCP12.1, on the other hand, induced a robust expression of IFN $\beta$ which was present at both time points in FR cells. IFN $\beta$ levels were also induced in C6 cells comparable to levels in FR cells at 6 hpi however this signal decreased tremendously by $12 \mathrm{hpi}$. Expression levels were normalized to the housekeeping gene, $\beta$-actin, however expression levels of $\beta$-actin were affected by infection in C6 glioma cells. As a result, all subsequent RT-PCR experiments used Cyclophilin B as the housekeeping gene as its expression levels were not affected by infection with wtVSV or rNCP12.1 (discussed in Appendix A). We next used quantitative RT-PCR (qRT-PCR) to obtain more precise expression levels of IFN $\beta$ in FR cells, primary astrocytes, and both glioma cell lines at 24hpi (Figure 3-3B). We found that both wtVSV and rNCP12.1 upregulated transcription of IFN $\beta$ however, $\mathrm{rNCP} 12.1$ increased message $3 \times 10^{3}$ fold greater than wildtype in primary astrocytes. rNCP12.1 also upregulated IFN $\beta$ in glioma cells $\sim 10^{2}$ fold over wildtype though these levels were exponentially lower than levels observed in primary astrocytes.

We next determined if upregulation of message following infection corresponded with an increase in production of active IFN through the use of an IFN bioassay. In this assay, L929 murine cells are exposed to harvested supernatant of immuno-stimulated cells and evaluated for inhibition of viral-induced CPE. We first tested the ability of our bioassay to detect IFN produced by the glial cells in response to polyinosinic: polycytidylic acid (pI:C), a synthetic dsRNA molecule used to elicit antiviral responses predominantly through the TLR3 pathway. Cells were either treated for 6 hours with $\mathrm{pI}: \mathrm{C}$ containing media or by transfection of $\mathrm{pI}: \mathrm{C}$ at increasing concentrations. After 6 hours, fresh media was added and samples were harvested at 24 hours following $\mathrm{pI}: \mathrm{C}$ treatment. Mouse embryonic fibroblasts (MEF) were used as a positive control (Figure 3-3C). Cells exposed to $\mathrm{pI}: \mathrm{C}$ containing media were unable to produce detectable IFN regardless of cell type at any concentration of pI:C. In line with what is known about $\mathrm{pI}: \mathrm{C}$ induced antiviral responses, transfection of $\mathrm{pI}: \mathrm{C}$ was superior in inducing an interferon response in all cell types tested, with the highest dose of $10 \mu \mathrm{g}$ eliciting the most robust production of IFN. PRAs produced the greatest amount of active IFN $\left(5.3 \times 10^{3} \mathrm{U} / \mathrm{mL}\right)$, similar to control cells, while F98 levels were less than $10^{2} \mathrm{U} / \mathrm{mL}$. We then followed the induction of IFN using this predetermined dose of transfected pI:C over a 24 hour time period in glioma cells. FR cells were used as the positive control for this study (Figure 3-3D). Starting at 4 hours, a gradual production of biologically active IFN in FR cells was observed with amounts greater than $10^{4} \mathrm{U} / \mathrm{mL}$ being produced by 24 hours after treatment. F98 glioma cells again produced the least amount of IFN throughout the study with levels less than $10^{2} \mathrm{U} / \mathrm{mL}$ throughout the time course. C6 glioma cells produced comparable amounts of active IFN to F98 glioma at earlier timepoints with an increase in production, exceeding $10^{2} \mathrm{U} / \mathrm{mL}$ by 24 hours.

Once the ability to produce active IFN detectable by our IFN bioassay was 
Figure 3-3. Cell and viral specific comparisons of IFN responses (A) Semi-quantitative RT-PCR and (B) qRTPCR verifying specificity of primers in FR and C6 cells. Cells were infected with either rNCP12.1 or wtVSV at an MOI of 10 for $1 \mathrm{~h}$ at $31^{\circ} \mathrm{C}$. At 6 and $12 \mathrm{hpi}$, cells were harvested, RNA was extracted using trizol reagent, and extracted RNA was amplified. rNCP12.1 infection resulted in increased IFN signal at both time points similarly in FR cells while signal increased in C6 cells at $6 \mathrm{~h}$ with dissipation of signal by $12 \mathrm{hpi}$. Results were normalized to the housekeeping gene, $\beta$ actin. Of note, changes in basal levels of $\beta$-actin were observed following viral infection C6 glioma cells, therefore expression levels of IFN $\beta 1$ were unable to be normalized in this cell line. (B) For qRT-PCR, expression levels were normalized to Cyclophilin B gene. Values are presented as means of duplicates with standard errors. (C) IFN activity following treatment with $(\mathrm{C}, \mathrm{D}) \mathrm{pI}: \mathrm{C}$ or $(\mathrm{E})$ virus. (C) Cells were either treated with various amounts $(\mu \mathrm{g})$ of $\mathrm{pI}: \mathrm{C}$ in culture media or with transfected $\mathrm{pI}: \mathrm{C}$ using lipofectamine 2000 (Invitrogen). At 24h after treatment, supernatant was collected and used for detection of IFN activity using an IFN bioassay in L929 murine cells. Values obtained are based on comparisons to antiviral activity of the rat IFN (rIFN) standard for each bioassay. IFN activity is expressed in units (U)/mL. (D) Detection of IFN activity following treatment of $10 \mu \mathrm{g}$ transfected $\mathrm{pI}: \mathrm{C}$ at $\mathrm{t}=2.5,4,6,12,18,24$ hours post transfection. There is a gradual increase in IFN activity starting at 4 hours posttreatment, extending to 24 hours. (E) Detection of IFN activity following viral infection. Cell lines were infected with wtVSV or rNCP12.1-GFP at an MOI of 10 for $1 \mathrm{~h}$ at $31^{\circ} \mathrm{C}$. At several time points following infection $(\mathrm{t}=6,12,18,24 \mathrm{hpi})$, supernatants were collected and residual virus was removed by ultrafiltration using Amicon filters (Millipore). Virus free supernatant was then used to detect IFN activity. (F) Ratio of IFN gene expression: IFN activity. Using data obtained from qRT-PCR and IFN bioassay described above, a combined graph was constructed revealing cellular and viral differences in the translation of IFN message into biologically active IFN. $\mathrm{pI}: \mathrm{C}=$ polyinosinic:polycytidylic acid; Level of significance denoted by “*” to "****" from low to very high significance, respectively. 
A

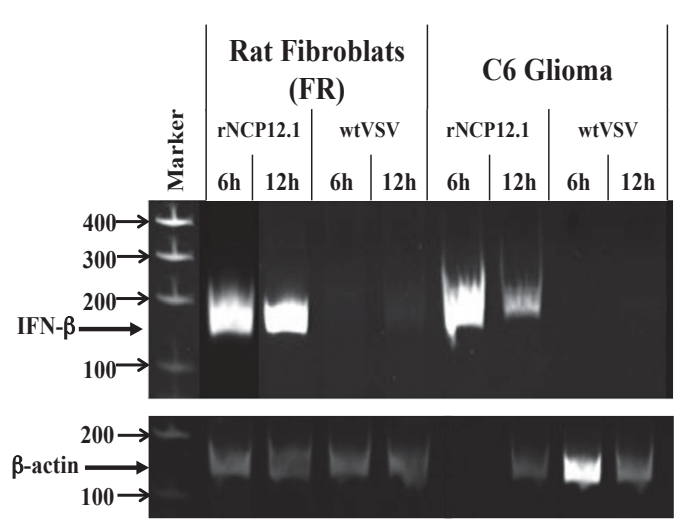

C

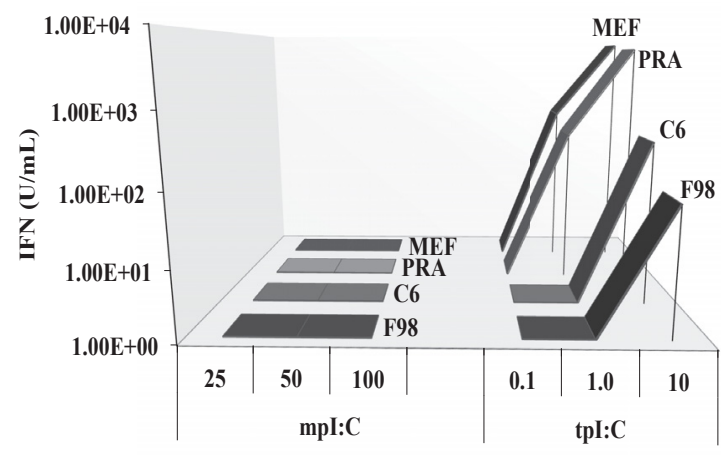

E

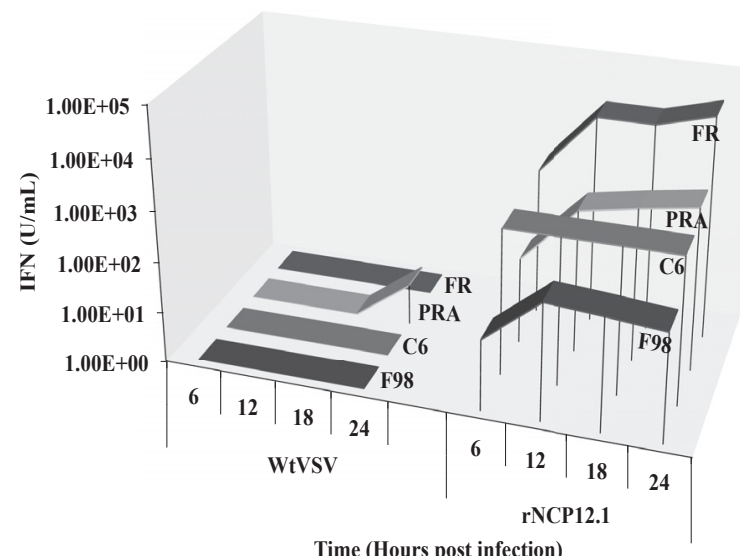

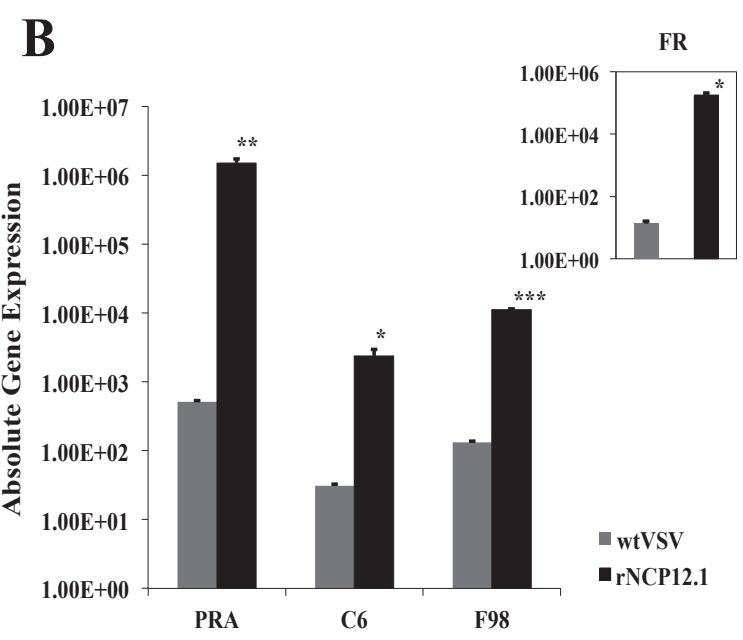

D

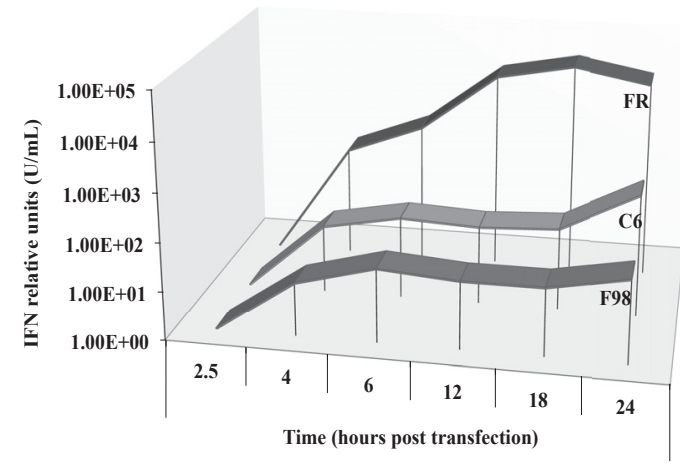

F

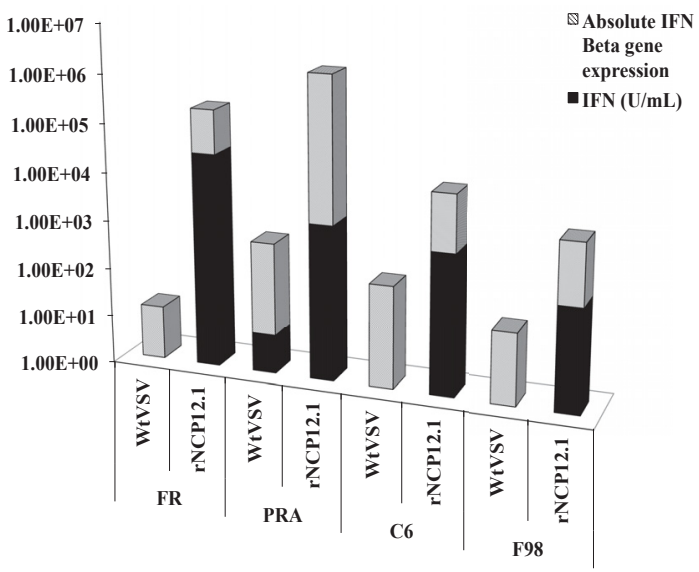


confirmed, we tested the supernatant from virally infected glial cells for presence of active IFN to determine if upregulation of IFN $\beta$ message corresponded with IFN activity.

Supernatant of virally stimulated cells was harvested from 6-24hpi and residual virus was separated by ultra-filtration so that IFN activity could be determined more accurately using the IFN bioassay (Figure 3-3E). We found that wtVSV infection did not result in detectable levels of IFN in the FR control cell line or in any glioma cell line, however a low amount of $6.25 \mathrm{U} / \mathrm{mL}$ IFN was detected at $24 \mathrm{hpi}$ in the primary astrocytes. Therefore, though wtVSV induced the synthesis of IFN $\beta$ message, this did not significantly translate into active protein in the glial cell lines. This observation is consistent with results from a number of studies that suggest matrix protein inhibits IFN responses at multiple levels of gene expression including nucleocytoplasmic transport of mRNA and protein translation. On the other hand, the rNCP12.1 matrix mutant stimulated the production of biologically active IFN in control cells, primary astrocytes, and glioma cell lines. FR cells produced copious amounts of IFN with levels totaling $2.56 \times 10^{4} \mathrm{U} / \mathrm{mL}$ at $24 \mathrm{hpi}$, comparable to induction of IFN gene expression in these cells. In comparison, though rNCP12.1-induced expression of IFN $\beta$ was 10 fold higher in primary astrocytes than FR cells, they produced a maximum of $1.6 \times 10^{3} \mathrm{U} / \mathrm{mL}$ of IFN at 24hpi, 16 fold less than FR cells. Not surprisingly, glioma cells produced active IFN during rNCP12.1 infection. F98-GFP produced the least active IFN at $24 \mathrm{hpi}\left(1.3 \times 10^{2}\right.$ $\mathrm{U} / \mathrm{mL}$ ) while C6s produced approximately 8 fold more IFN than F98 at the same time point despite its lower induction of IFN $\beta$ transcription. In addition, IFN levels in C6s remained steady, maintaining $800 \mathrm{U} / \mathrm{mL}$ of IFN over all time points. This steady state of IFN produced by $\mathrm{C} 6$ cells corresponds to the stagnant viral yield and delayed viralinduced cytotoxicity by rNCP12.1 infection over the same time period. In comparison to primary astrocytes, this amount was approximately 16 fold higher at the earliest time point tested (6hpi) however IFN production in PRAs, eventually increased to surpass C6 levels by $18 \mathrm{hpi}$. These results suggest that not only is the quantity of active IFN produced important in eliciting a protective antiviral state in infected cells during viral replication but the ability to amplify IFN signaling is important as well.

\section{IFN $\beta$ Gene Expression Does Not Correlate with Production of Active IFN in Primary Rat Astrocytes}

Based on a recent review by Vogel and Marcotte, $40 \%$ of the variability in protein synthesis can be attributed to the abundance of mRNA. The other $60 \%$ is due to posttranscriptional events such as protein stability or regulatory mechanisms of translation [370]. Generally, message in mammalian cells is less stable and is produced at a much lower rate than proteins. The average rate of synthesis of transcripts has been shown to be approximately two mRNA per hour compared to dozens of protein molecules/mRNA/h [370]. This rate tends to vary based on cell type, protein of interest (metabolic proteins versus proteins involved in transcriptional regulation), and cellular conditions. Nevertheless, most studies use mRNA abundance as a means of predicting the likelihood of detecting its coded protein. In our studies, all three parameters appear to play a role in the correlation between mRNA abundance and protein activity. It must be 
noted, however, that these studies do not aim to correlate mRNA message with the total amount of protein produced but rather to the amount of active protein produced under variable conditions. A comparison of IFN gene expression to production of active IFN following mutant and wildtype infection revealed notable cell and viral specific differences in the ability to elicit the IFN mediated antiviral pathway. In each cell type, though wtVSV induced gene expression of IFN $\beta$ over non-infected cells, this did not correlate with production of active IFN. rNCP12.1 however induced expression of IFN $\beta$ which translated to active IFN, though still not according to the expected rate of synthesis. Interestingly, this deviation was more pronounced in primary astrocytes. Whereas the ratio of active IFN to message corresponded to approximately 1:10 in the control cell line and in both glioma cell lines, values were more disproportionate in primary astrocytes with a ratio closer to $1: 10^{3}$ at 24 hpi (Figure 3-3F).

In evaluating the IFN response from this perspective, these results not only reinforce the influence of the matrix protein in the antiviral response pathway but they also confirm that a number of variables effect adherence to the rate of production of mRNA to protein. We specifically see the effects of cell type as primary astrocytes produced much lower amounts of active IFN relative to its level of induction of gene expression following rNCP12.1 infection. Despite this disparity, PRAs demonstrated a greater response to the active IFN produced which was evident not only in cell viability but in the production of infectious virus over time. On the other hand, though C6 glioma cells produced detectable amounts of active IFN following rNCP12.1 infection that correlated more with the induction of IFN $\beta$ gene expression, these cells responded less to IFN which was also evident in cell viability, cell rounding, and viral growth kinetics.

\section{Primary Astrocytes Are Highly Sensitive to the Anti-Viral Effects of IFN While Glioma Cells Are Not}

In order to determine if differences in the ability to produce IFN correlated with IFN sensitivity in glial cell lines, we performed a protection assay in which cells were pretreated with escalating doses of rat IFN $\beta$ (r IFN $\beta$ ) (0-1000 units) for 24 hours then infected with wtVSV at an MOI of 3. At 24hpi, cells were stained by immunofluorescence to detect VSV nucleocapsid (N). Response to interferon was graded by the gross detection of staining in IFN treated versus untreated cells. Protection was defined as samples with less than 50\% VSV-N positive cells (Table 3-1).

Primary astrocytes were protected starting at $3.125 \mathrm{U}$ of IFN. C6s were also protected from viral infection but at higher doses of IFN, starting close to 100U. F98s, however, were not protected from viral infection even at the highest dose of 1000 units (Table 3-1 and Figure 3-4). These results suggest the glioma cell lines are less sensitive to the antiviral effects of IFN and confirm the results of previous studies that the level of IFN sensitivity can vary among tumor cell types even when derived from the same histological cell of origin [358, 371]. 
Table 3-1. Sensitivity to antiviral effects of IFN

\begin{tabular}{|c|c|c|c|c|c|c|c|c|c|c|c|c|}
\hline \multirow{3}{*}{$\begin{array}{c}\text { Cell } \\
\text { Type }\end{array}$} & \multicolumn{12}{|c|}{ IFN (U) } \\
\hline & \multicolumn{4}{|c|}{ High Dose } & \multicolumn{8}{|c|}{ Low Dose } \\
\hline & 1000 & 250 & 125 & 62.5 & 100 & 50 & 25 & 12.5 & 6.25 & 3.125 & 2.562 & 1.562 \\
\hline PRA & + & + & + & + & + & + & + & + & + & + & + & - \\
\hline C6 & + & + & + & - & + & - & - & - & - & - & - & - \\
\hline F98 & - & - & - & - & - & - & - & - & - & - & - & - \\
\hline
\end{tabular}

Note: + , protection $(<50 \%$ cells positive for $\mathrm{N}$ protein staining); -, no protection $(>50 \%$ cells positive for $\mathrm{N}$ protein staining). 


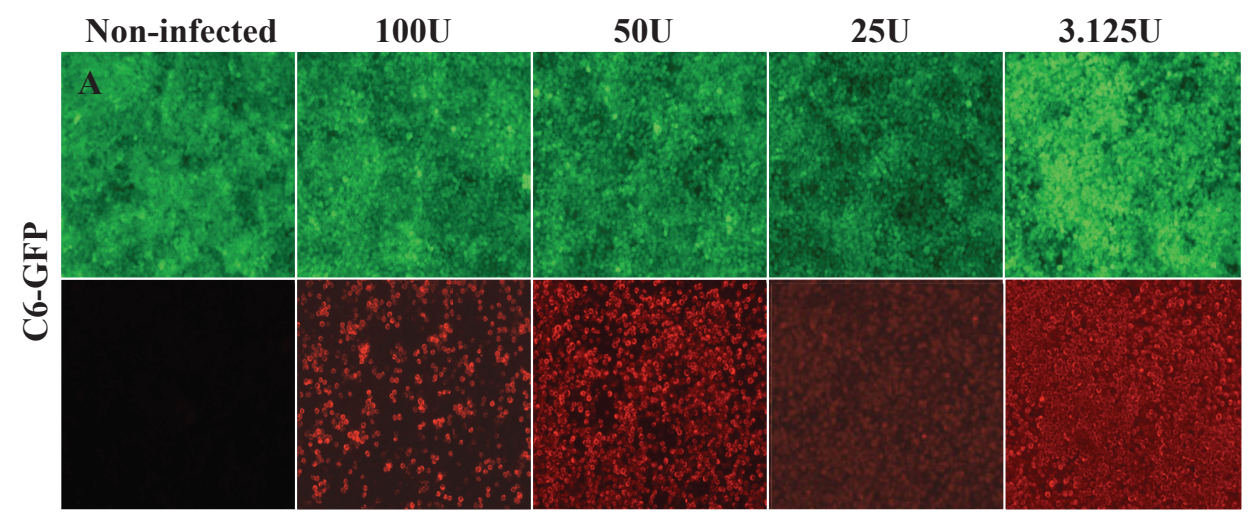

Tumor-

GFP

$\alpha-V S V ~ N$

Rhodamine

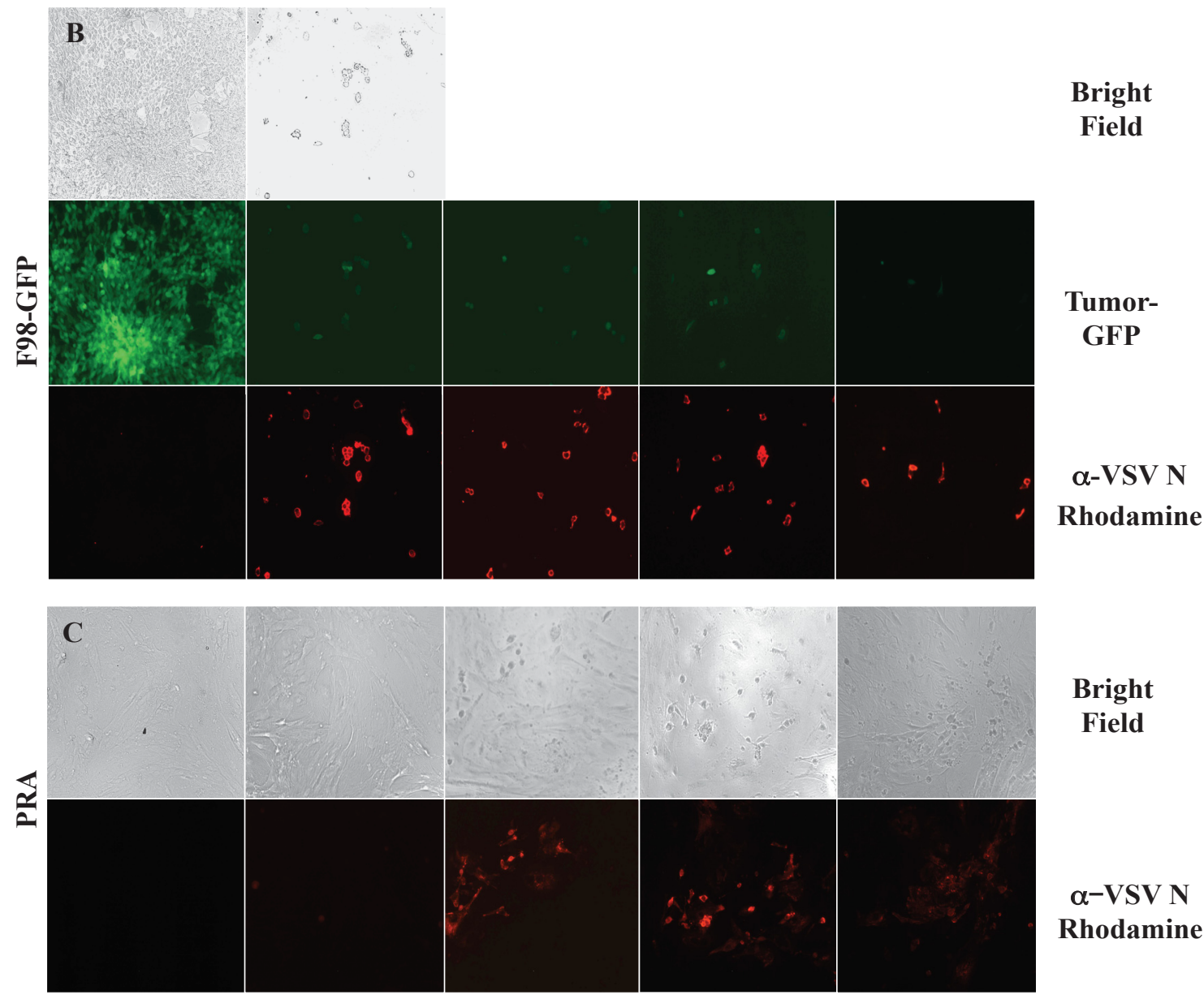

Figure 3-4. Representative fluorescence micrographs showing infection of cells with wtVSV following IFN pre-treatment

(A) C6-GFP, (B) F98-GFP, and (C) Primary rat astrocytes were pre-treated with various doses of IFN then infected with wtVSV at an MOI of 3. Cells were then labeled with anti-VSV N antibody conjugated to Rhodamine at 24hpi and visualized by fluorescence microscopy at $10 \mathrm{X}$ magnification. 
As the above experiments have demonstrated, matrix mutations in rNCP12.1 alter its ability to cause cell rounding and apoptosis in normal cells, which appears to be correlated with its ability to promote IFN $\beta$ gene expression and, ultimately, IFN production. On the other hand, these defects reveal a spectrum of phenotypes in tumor cells of glial origin with F98 displaying a high IFN resistance, high CPE phenotype and C6 glioma cells displaying a more intermediate IFN resistance with an intermediate CPE phenotype.

\section{IFN Activity Following rNCP12.1 Infection Is Associated with Induction of ISGs in Primary Astrocytes While Glioma Cells Vary in Their Ability to Elicit Downstream IFN Responses}

In order to evaluate involvement of specific elements of the IFN pathway and how they correlate with cytopathic effects and IFN production in tumor cells, we surveyed the expression of interferon stimulated genes by qRT-PCR at 24 hours following viral infection using the cell fraction collected from the previous IFN bioassay studies. Key ISGs that have been shown to be elevated following exposure to IFN and/or viral infection were considered [289, 333, 371, 372]. According to data obtained by Stojdl et al [289], expression of ISGs are time specific following VSV infection and this expression may vary depending on alterations in the matrix protein. For instance, primary transcriptional responders appear during early infection (3-6hpi). These include ISG15 and IFN $\beta$ and are dependent on factors that bind interferon stimulated response elements (ISRE) in the IFN $\beta$ promoter region such as IRF3 and NFKB. IRF-1 and RIG-I have been shown in separate studies to be upregulated early in infection and may therefore be considered primary transcriptional responders. In addition, these two ISGs have been shown to be constitutively active in a number of cell lines including astrocytes $[310,372]$ and are directly upregulated by viral infection even in the absence of interferon $[310,373]$. Mx-1/2, GBP-1, and Plscr-1 are considered secondary transcription responders, appearing 6-12 hpi. These responders are dependent on the activity of ISGF3, a heterotrimer formed by IRF9, Stat 1, and Stat2 as has been described. Lastly, responders such as IFN $\alpha 4$ appear latest in infection, usually after 12 hours. These genes are known as tertiary transcription responders and are dependent on IRF7 for their activation [289]. Other studies, however, consider IFN $\alpha 4$ an immediate early response gene, showing upregulation as early as 4hpi even in the absence of active protein synthesis [374]. Nevertheless, expression of secondary responders is inhibited by wtVSV infection whereas mutants defective in inhibition of host gene expression promote expression of these responders. By blocking this step, wtVSV consequently inhibits the transcription of tertiary responders as well.

As expected, rNCP12.1 induced the expression of all ISGs over wildtype in primary astrocytes, similar to induction in FR cells (Figure 3-5A-F). Of the ISGs tested, only plscrl was not significantly induced by infection and no significant differences in expression levels were observed between wtVSV and rNCP12.1 (Figure 3-5F). Glioma cell lines were more variable in their ISG expression. F98 glioma cells are both resistant to IFN and unable to produce amounts of active IFN that would be protective against 
Figure 3-5. Differences in interferon stimulated genes (ISGs) following viral infection with wtVSV or rNCP12.1-GFP

(A) IFNa4, (B) Ddx-58 (RIG-I), (C) IRF-1, (D) Mx1/Mx2, (E) GBP-1, (F) Plscr-1. Cells were infected at an MOI of 10 for 1 hour at $31^{\circ} \mathrm{C}$. Cells were then harvested, RNA was extracted, amplified, and quantified using qRTPCR at 24 hours of infection. Expression levels are normalized to the cellular Cyclophilin B gene and are presented as means of duplicates \pm SD. Expression levels vary based on gene tested (see y axis for different scales) 


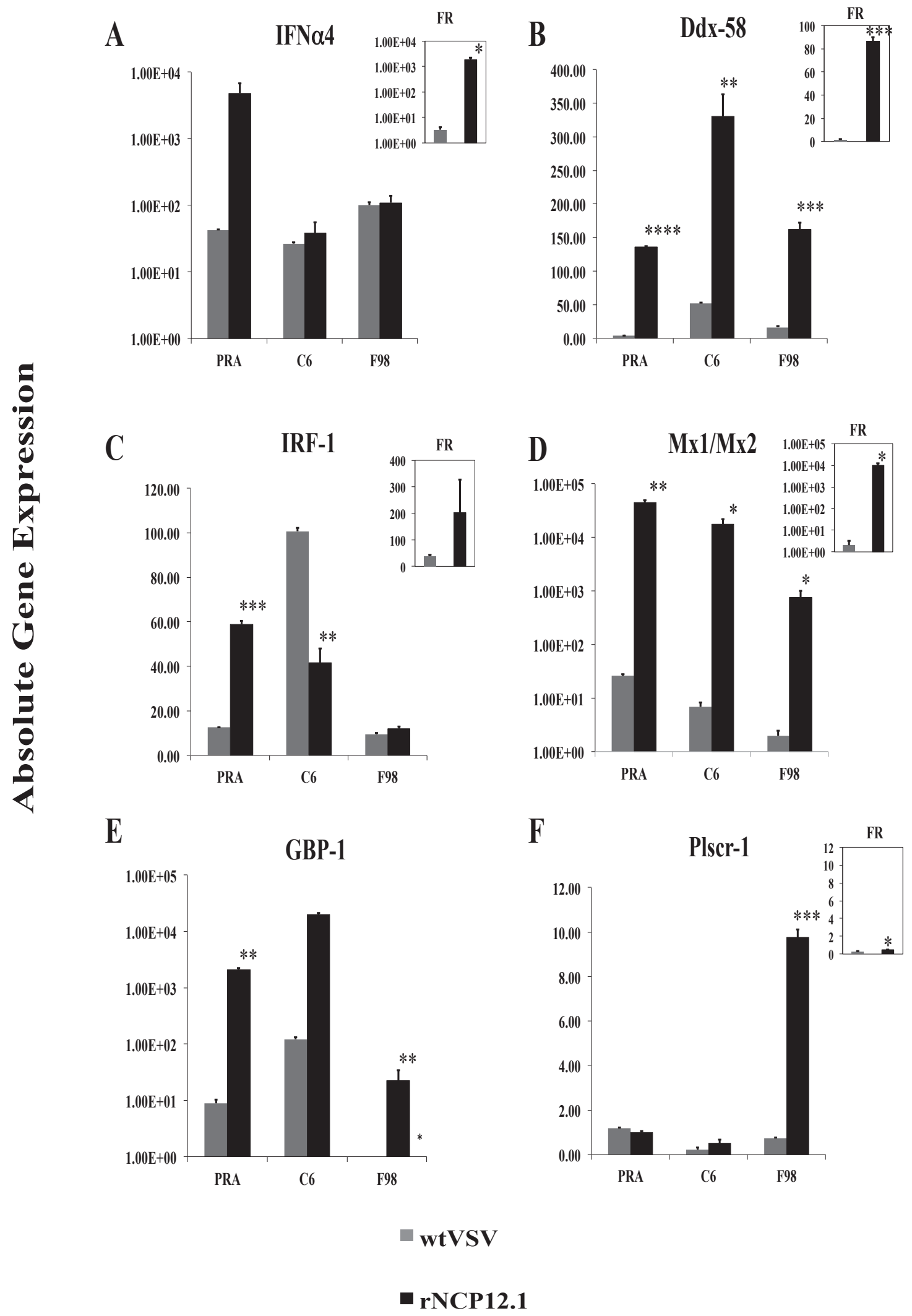


viral infection. In parallel, the induction of most ISGs tested was lowest in F98s following infection compared to other cell types. rNCP12.1 upregulated all secondary ISG responders significantly over wtVSV (Figure 3-5D-F). Interestingly, plscr1, a secondary responder that was not induced in the other cell lines, was expressed approximately 10 fold higher after rNCP12.1 infection relative to wtVSV in F98 and to expression in the other cell lines (Figure 3-5F). In addition, expression of the primary responder, IRF1, and tertiary responder, IFN $\alpha 4$, were induced by both viruses at similar levels in the glioma cell lines. C6 glioma cells, having a more intermediate resistance to IFN, also varied in ISG expression. Both GBP1 and Mx1/2, secondary ISGs, were induced by rNCP12.1 infection similar to induction in PRAs although the induction of GBP1 was not significant. Like PRAs, these cells were low in their induction of plscr1 with both wtVSV and rNCP12.1 (Figure 3-5D-F). Unexpectedly, IRF1 induction did not follow the pattern of higher induction with rNCP12.1 infection. Rather, wtVSV induced IRF-1 expression 2.5 fold higher than rNCP12.1 (Figure 3-5C). Another primary responder, Ddx58, had a greater induction in C6s after rNCP12.1 infection with expression approximately 2.5 fold higher than rNCP12.1 infection in primary astrocytes (Figure 3-5B). These data are represented by a heat map depicted in Figure 3-6.

\section{rNCP12.1 Alters the Temporal Expression of Ddx-58 (RIG-I) in Glial Cells}

Ddx58 or RIG-I is important in the detection of viral RNA and therefore in eliciting IFN mediated antiviral responses during infection. In addition, it is a known ISG that is upregulated early during infection. Ddx-58 is also a known activator of NFKB [372] that has been implicated as a determinant in resistance to the apoptotic effects of IFN. Like IRF-1, Ddx-58 was found to be constitutively expressed in primary human astrocytes with increased levels following wtVSV infection reaching optimal expression by 4 hpi then falling back to baseline by 8hpi [372]. Our results demonstrate that Ddx-58 expression is low but slightly elevated at 24 hours following wtVSV infection in both rat fibroblasts and primary rat astrocytes (Figure 3-5B). Both glioma cells showed higher expression following wtVSV infection with C6 expression 50 fold higher than uninfected cells. There was an even greater rise in expression in both glioma and normal cells following rNCP12.1 infection with levels more than 100 fold higher in all cell types over uninfected cells. As with wtVSV infection, rNCP12.1 induced expression of Ddx58 in C6 glioma that was double the levels of induction in other cell types. In comparison to results by Furr et al [372], our results showed that Ddx 58 expression was still elevated however at low levels even at $24 \mathrm{hpi}$ and this expression was greatly enhanced by the matrix mutations found in rNCP12.1. We also see that in C6 glioma, which demonstrated stagnant viral yield, fixed cell viability, and unwavering levels of active IFN during the first 24-48 hours of rNCP12.1 infection, Ddx58 levels are highly elevated even more so than normal cells. It could be that the upregulation of Ddx58 in these cells is involved in delaying apoptosis during the first 2 days of infection via downstream Ddx58 stimulated effectors.

Though there are obvious host specific differences in IFN responses following rNCP12.1 infection, the overall inability of glioma cells to produce a sustainable antiviral 


\begin{tabular}{|c|c|c|c|c|c|c|c|c|}
\hline \multirow[t]{2}{*}{$\begin{array}{l}\text { IFN Stimulated } \\
\text { Genes (ISGs) }\end{array}$} & \multicolumn{2}{|c|}{ Rat Fibroblasts } & \multicolumn{2}{|c|}{ Primary Rat Astrocytes } & \multicolumn{2}{|c|}{ F98-GFP Rat Glioma } & \multicolumn{2}{|c|}{ C6-GFP Rat Glioma } \\
\hline & wtVSV & rNCP12.1 & wtVSV & rNCP12.1 & wtVSV & rNCP12.1 & wtVSV & rNCP12.1 \\
\hline IFN- $\beta 1$ 1* & $1.3 \times 10^{1}$ & $1.8 \times 10^{5}$ & $5.1 \times 10^{2}$ & $1.5 \times 10^{6}$ & $1.3 \times 10^{2}$ & $1.1 \times 10^{4}$ & $3.0 \times 10^{1}$ & $2.4 \times 10^{3}$ \\
\hline IRF-1 & $3.8 \times 10^{1}$ & $2.0 \times 10^{2}$ & $1.3 \times 10^{1}$ & $5.9 \times 10^{1}$ & $10^{1}$ & $1.1 \times 10^{1}$ & $10^{2}$ & $4.1 \times 10^{1}$ \\
\hline $\begin{array}{l}\text { Ddx-58 } \\
(R I G-I)\end{array}$ & $1.7 \times 10^{0}$ & $8.6 \times 10^{1}$ & $4 \times 10^{0}$ & $1.3 \times 10^{2}$ & $1.6 \times 10^{1}$ & $1.6 \times 10^{2}$ & $5.2 \times 10^{1}$ & $3.3 \times 10^{2}$ \\
\hline Mx-1/2 & $1.9 \times 10^{0}$ & $9.9 \times 10^{3}$ & $2.6 \times 10^{1}$ & $4.5 \times 10^{4}$ & $2 \times 10^{0}$ & $7.7 \times 10^{2}$ & $6.8 \times 10^{0}$ & $1.7 \times 10^{4}$ \\
\hline GBP-1 & $\mathrm{N} / \mathrm{A}$ & N/A & $8.7 \times 10^{0}$ & $2 \times 10^{3}$ & $<1$ & $2 \times 10^{1}$ & $1.2 \times 10^{2}$ & $2 \times 10^{4}$ \\
\hline Plscr-1 & $<1$ & $<1$ & $10^{\circ}$ & $10^{\circ}$ & $<1$ & $10^{1}$ & $<1$ & $<1$ \\
\hline IFN- $\alpha 4$ & $3.3 \times 10^{0}$ & $1.8 \times 10^{3}$ & $4.2 \times 10^{1}$ & $4.6 \times 10^{3}$ & $10^{2}$ & $10^{2}$ & $2.7 \times 10^{1}$ & $3.7 \times 10^{1}$ \\
\hline
\end{tabular}

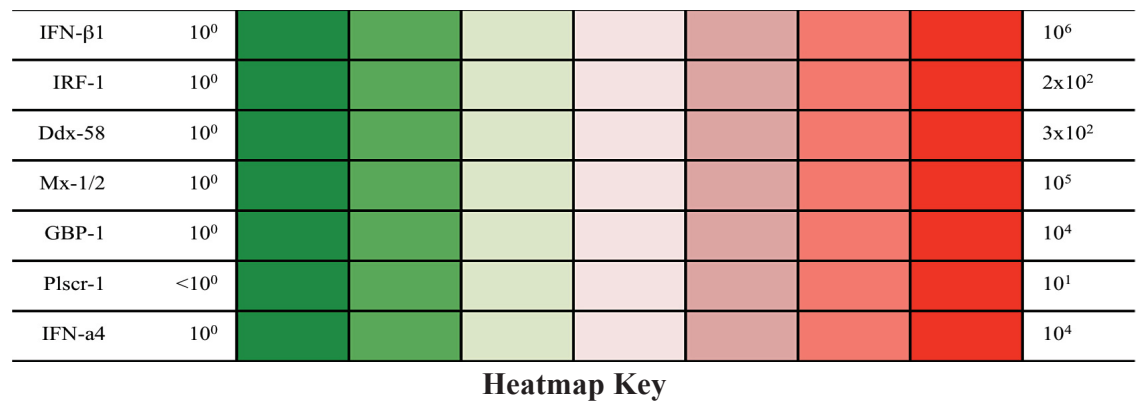

Figure 3-6. Heat map representation of IFN stimulated gene (ISG) expression in normal and tumor glial cells following viral infection

$\mathrm{N} / \mathrm{A}=$ not available for comparison 
IFN response in comparison to the protective antiviral responses elicited in normal cells contributes to the oncolytic capacity of this vector. Based on these differences, we next evaluated the oncolytic activity of rNCP12.1 in an in vivo model of intracranial glioma.

\section{Efficacy of rNCP12.1 in an Immunocompetent Intracranial Model of Glioma}

\section{Intracranial Administration of rNCP12.1 Is Safe Relative to wtVSV in Non-Tumor Bearing Animals}

The mortality and morbidity caused by VSV infection varies based on immune status of host as well as mode of administration. This has been studied using experimental murine models in which intranasal administration of VSV leads to a less fatal encephalitic disease whereas intracranial administration of virus usually results in a fatal disease marked by severe neurological symptoms within 2 weeks post-infection [219, 351, 375]. Younger animals are more susceptible to viral neurotoxicity; however, attenuated strains of VSV demonstrate mild disease regardless of age. Hence, we wanted to first determine mortality and morbidity in animals receiving rNCP12.1 intracranially relative to wildtype. This was carried out in an immunocompetent non-tumor bearing model. On day zero, six week old Wistar rats weighing $\sim 250-350$ grams were given an intracranial injection of $10^{9}$ pfu rNCP12.1-GFP or wtVSV-GFP. Along with survival, weight trends were followed throughout the study. Animals were sacrificed at day 15 post-infection or when criteria for euthanasia were met. As expected, we found that wtVSV caused severe neurotoxicity with all animals succumbing to infection by 8 days post-infection. On the other hand, neurotoxicity of rNCP12.1 was attenuated with animals surviving to the end of the study. In addition, average weight of rNCP12.1infected animals progressed at a comparable rate to animals receiving only viral vehicle (Tris-Saline $+10 \%$ sucrose, TN sucrose) (data not shown). Based on these studies we concluded that rNCP12.1 could safely be administered intracranially for the treatment of glioma in this rat model.

\section{rNCP12.1 Decreases Tumor Load in a Dose Dependent Manner Without Causing Morbidity in an Intracranial Model of C6-GFP Glioma}

Our initial evaluation of the oncolytic activity of rNCP12.1 against intracranially implanted glioma was first executed in a rat model using C6-GFP glioma cells in the immunocompetent Wistar rat. This model was chosen as previous studies by our lab demonstrated in vitro proof-of-principle of the oncolytic activity of a replication restricted rVSV vector in a co-culture of C6 glioma with normal CNS cells from various brain structures [354]. On day zero, cells were implanted intracranially as described and allowed to grow for three days. At three days post tumor implantation, animals were given varying doses of intratumorally injected virus. Animals were then euthanized at 15 days post infection in order to assess differences in tumor size based on fluorescence from GFP expressing tumor compared to animals treated with viral vehicle (TN-sucrose). 
Tumor sizes were measured from cryo-sections by circumscribing the tumor area defined by GFP fluorescence using NIH ImageJ software as described in Chapter 2.

Measurements based on fluorescence were compared to those obtained by H\&E staining of tissue and found to be indistinguishable (data not shown).

We found that tumor size inversely correlated with dose of VSV. The highest dose tested of $10^{8} \mathrm{pfu}$ demonstrated the greatest decrease in tumor load of $82 \%$ relative to vehicle treated tumors while the lowest dose of $10^{5} \mathrm{pfu}$ resulted in $15 \%$ tumor load reduction (Figure 3-7A). Surprisingly, three of five animals treated with $10^{8} \mathrm{pfu}$ had nearly undetectable tumors. In addition, weight trends were followed in animals receiving the highest dose of $\mathrm{rNCP} 12.1\left(10^{7}\right.$ and $\left.10^{8} \mathrm{pfu}\right)$. Weights decreased accordingly in all groups following tumor implantation but gradually increased over the next few days before virus administration. On the day following treatment, all animals decreased in weight with animals receiving a higher dose of virus decreasing the most over the first 24 hours of infection (5.2g versus $1.2 \mathrm{~g}$ in vehicle only). The animals in this treatment group also experienced an extended duration of weight decline seen on day 5 , whereas, an increase in weight was observed in the other two groups at this time point. However, by day six, average weights of all groups rose at similar rates though animals receiving the highest dose of virus had an overall lower average weight at each time point. Despite this, no group experienced more than a $5 \%$ decrease in weight during the study and by day 15, all animals had gained at least 50 grams over their pre-tumor implantation weight (Figure 3-7B). Together, these results suggest that one dose of rNCP12.1 does not cause significant morbidity or mortality as all animals survived to the end of the study with an overall increase in weight over time. Therefore this oncolytic virus appeared to be safe for administration in the brains of tumor bearing animals. In addition, we concluded a single dose of rNCP12.1 was effective at reducing tumor growth in a dose-dependent manner when given intracranially in the C6 glioma bearing Wistar rat model.

\section{Comparison of the F98 Glioma/ Fischer Rat Model}

Because C6 gliomas are derived from outbred Wistar rat, they have been shown to be immunogenic in inbred rats causing tumors to often regress spontaneously by 4 weeks post-implantation. As a result, this model, though appropriate for short term studies, is not appropriate for studies in which long term survival would be determined [376, 377]. Therefore, the syngeneic F98 glioma/ Fischer rat model was used for the remainder of our studies. The F98 glioma cell line was chemically induced by giving the mutagenic alkylating agent, ethylnitrosourea (ENU), to pregnant Fischer rats resulting in offspring harboring spontaneous brain tumors $[378,379]$. F98 tumors are refractory to most therapies and demonstrate an invasive pattern of growth. As few as 10-100 tumor cells are capable of developing into an aggressive tumor and, with an engraftment rate of $>90 \%$ following intracranial administration, this model is considered a reliable experimental model capable of mimicking human disease [376, 380]. Further, time of progression from implantation to adverse neurological changes caused by tumor mass effect is short, occurring in about 2 weeks post-implantation. Therefore this model is 
Figure 3-7. Dose-dependent reduction of C6 tumors after rNCP12.1 treatment C6-GFP glioma bearing adult Wistar rats were inoculated with a single dose of intratumoral (i.t.) virus on day 3 post tumor implantation (pti). Four treatment groups included a single injection of rNCP12.1-GFP at $10^{5}, 10^{6}, 10^{7}$, or $10^{8} \mathrm{pfu}(\mathrm{n}=5)$. Animals were euthanized at day $15 \mathrm{pti}$ and tumor load was determined based on tumor measurements from fluorescence images using NIH ImageJ software. (A) Tumor size is represented by percent (\%) reduction compared to control tumors. (B) Weight trends of animals treated in higher dose $\left(10^{7}\right.$ and $\left.10^{8} \mathrm{pfu}\right)$ of rNCP12.1 compared to animals in control (vehicle) group. Weights at each time point are an average of those obtained for each treatment group. Unpublished data from Himangi Jayakar and Qihong Zhou, 2005. 


\section{A}
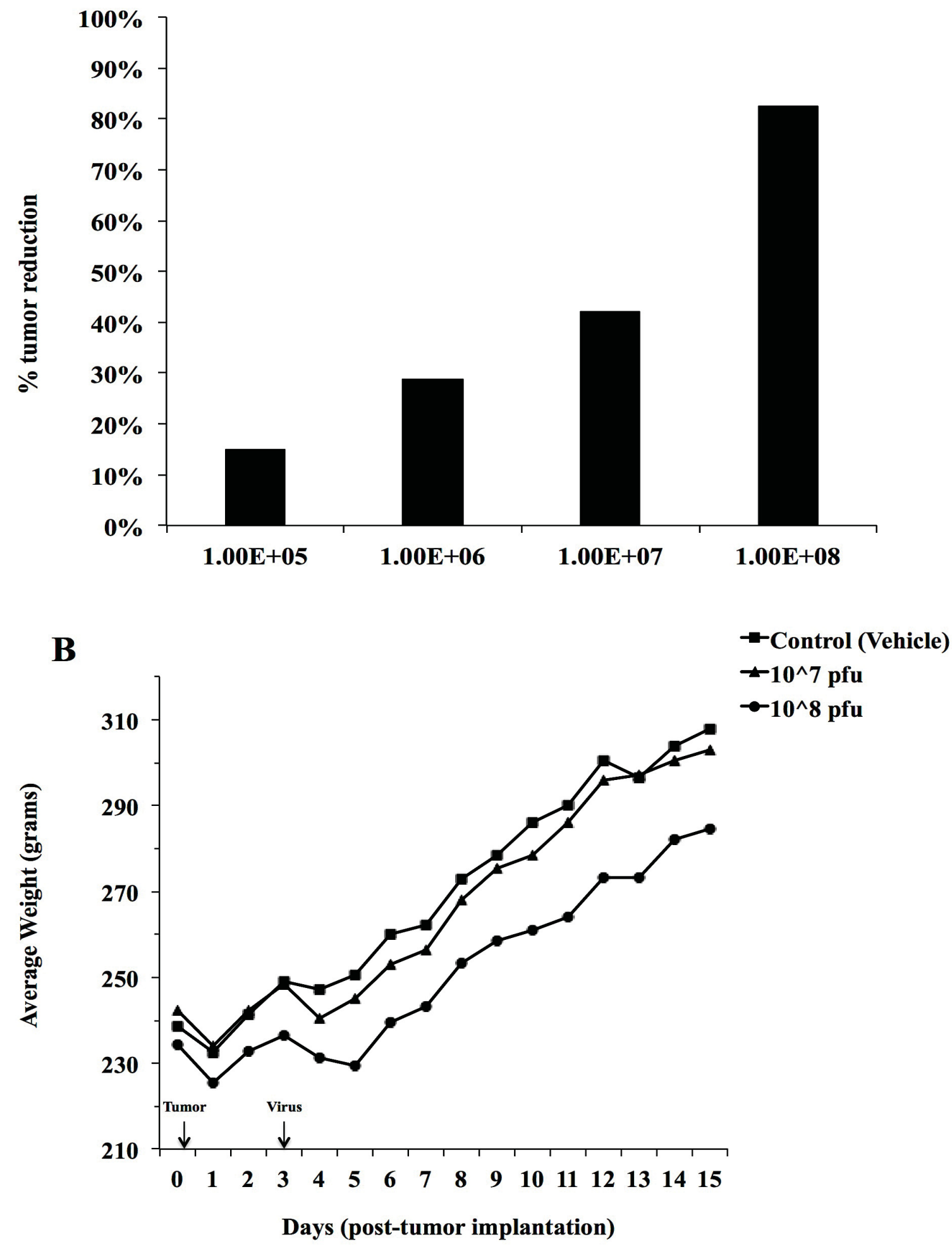
effective at allowing us to study survival as well as the possible immunological responses elicited against virus and/or against tumor as a result of viral treatment.

We carried out a comparative study of tumor anatomy and histology between the C6-Wistar rat model and F98-Fischer rat model. F98 glioma cell line was previously transduced with the $\mathrm{pFB}$ retrovirus expressing GFP (pFB-GFP) to allow visualization of tumor. As described, either $10^{5} \mathrm{C} 6$ glioma cells or F98 glioma cells were implanted intracranially in Wister or Fischer rat, respectively. On day 15, post-tumor implantation, animals were euthanized and brains were harvested for analysis of tumor load following H\&E staining and fluorescence microscopy for visualization of GFP expressing tumors. Representative images in Figure 3-8 demonstrate the gross and microscopic features of these tumors. While $\mathrm{C} 6$ gliomas are less aggressive and more immunogenic, forming a well-demarcated tumor mass at 15 days following implantation (Figure 3-8A-C), F98 gliomas are more infiltrative and tend to invade surrounding normal tissue much like high-grade human gliomas (Figure 3-8D-F).

We next sought to determine the growth kinetics of implanted F98 tumors to gauge changes in tumor size over several time points and the reproducibility of establishing tumors following implantation (Figure 3-9A). $10^{5}$ cells were implanted intracranially as decribed and animals were euthanized at 3, 6, 9, and 15 days post tumor implantation. Brains were harvested and assessed for tumor load based on GFP expression of F98 glioma cells. We found that establishment of tumors were reproducible at each time point and that tumor size gradually increased as expected over time. Measure of morbidity was determined not only by weight trends throughout the study but also by changes in neurological function as assessed by the rat coma scale (described in Chapter 2). In this study, average weight of animals never dropped below $10 \%$ of starting weight (Figure 3-9B) and neuroscores ranged between 9.5-11 with the lowest scores occurring at day 12 (Figure 3-9C). This range of neuroscores is expected as tumor load usually begins to cause neurological dysfunction within 2 weeks of implantation. As experienced in this study, there are often additional neurological signs and symptoms not measurable with the rat coma scale that may occur. These additional neurological signs, when observed, range from changes in grooming to staggering during ambulation (ataxia). Based on symptomology, animals may require euthanasia even when neuroscores and weights are within normal limits. An explanation of these additional symptoms that are considered in criteria for euthanasia can be found in Chapter 2.

\section{Intracranial Administration of rNCP12.1 Is Safe but Results in a Non-Durable Reduction of Tumor Load in the F98 Glioma Model}

With reproducibility of tumor implantation and growth established, we next tested the oncolytic activity of rNCP12.1 against F98 glioma in vivo in order to determine if the antitumor activity we observed in our in vitro studies translated into the intracranial animal model. Because the highest dose tested in C6-Wistar model of $10^{8}$ pfu was well tolerated and most effective, we decided to increase the dose to $10^{9} \mathrm{pfu}$ for subsequent 

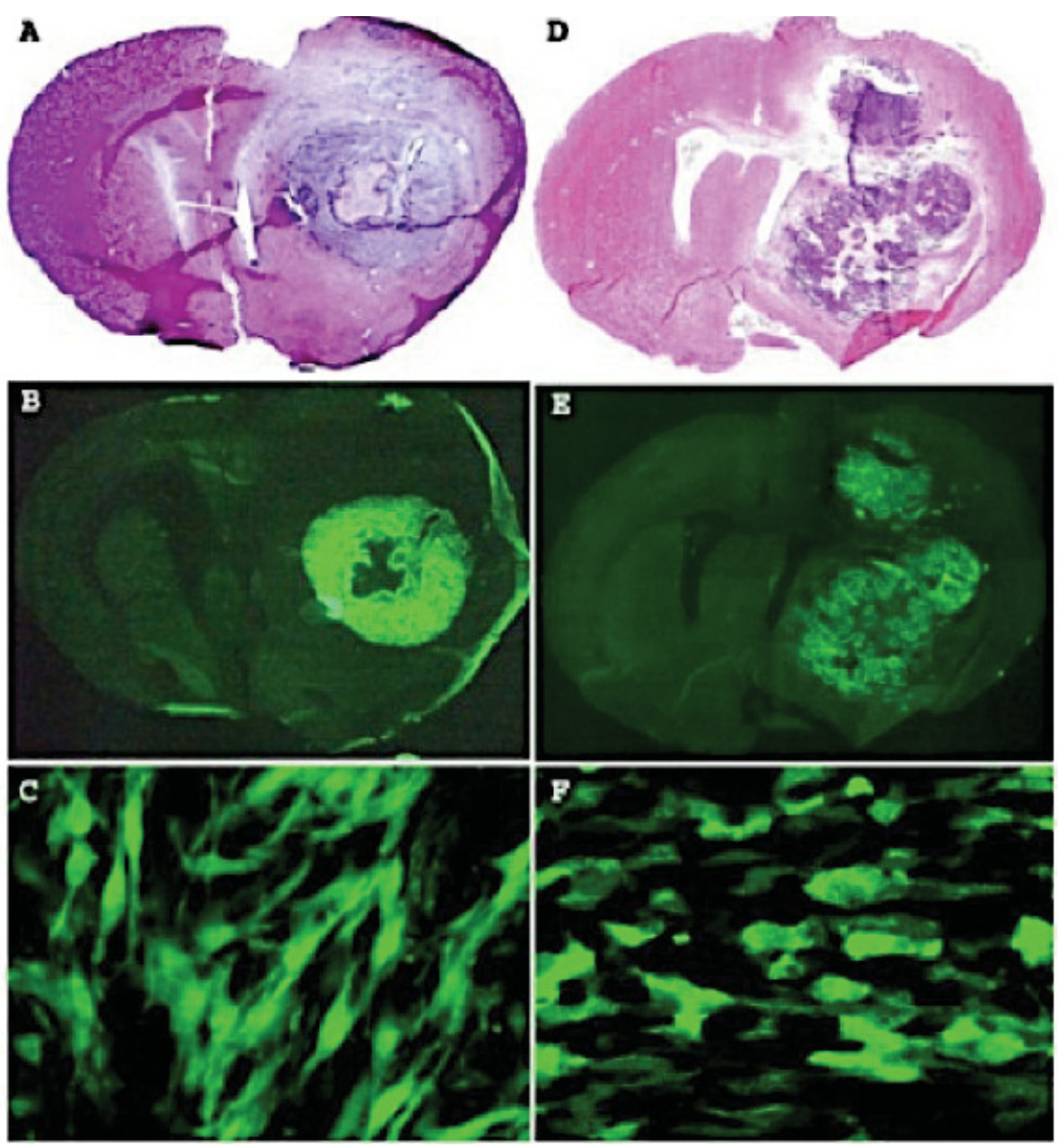

Figure 3-8. Comparison of the $\mathrm{C6}$ and $\mathrm{F98}$ rat intracranial glioma models

Coronal sections of brain tissue harboring (A-C) C6 and (D-F) F98 intracranial gliomas in Wistar and Fischer rats, respectively. (A, D) H\&E stained sections taken at 1.25X magnification. Brain tissue in the right hemisphere reveals increased cellular infiltration and a tumor mass that is causing compression of the ventricles and a midline shift toward the contralateral side of the brain. (B\&E) Fluorescence microscopy illuminating the GFP (green) expressing tumor. (C, F) Fluorescence confocal microscopy at 60X magnification of both C6 (C) and F98 (F) gliomas within brain tissue. 
A

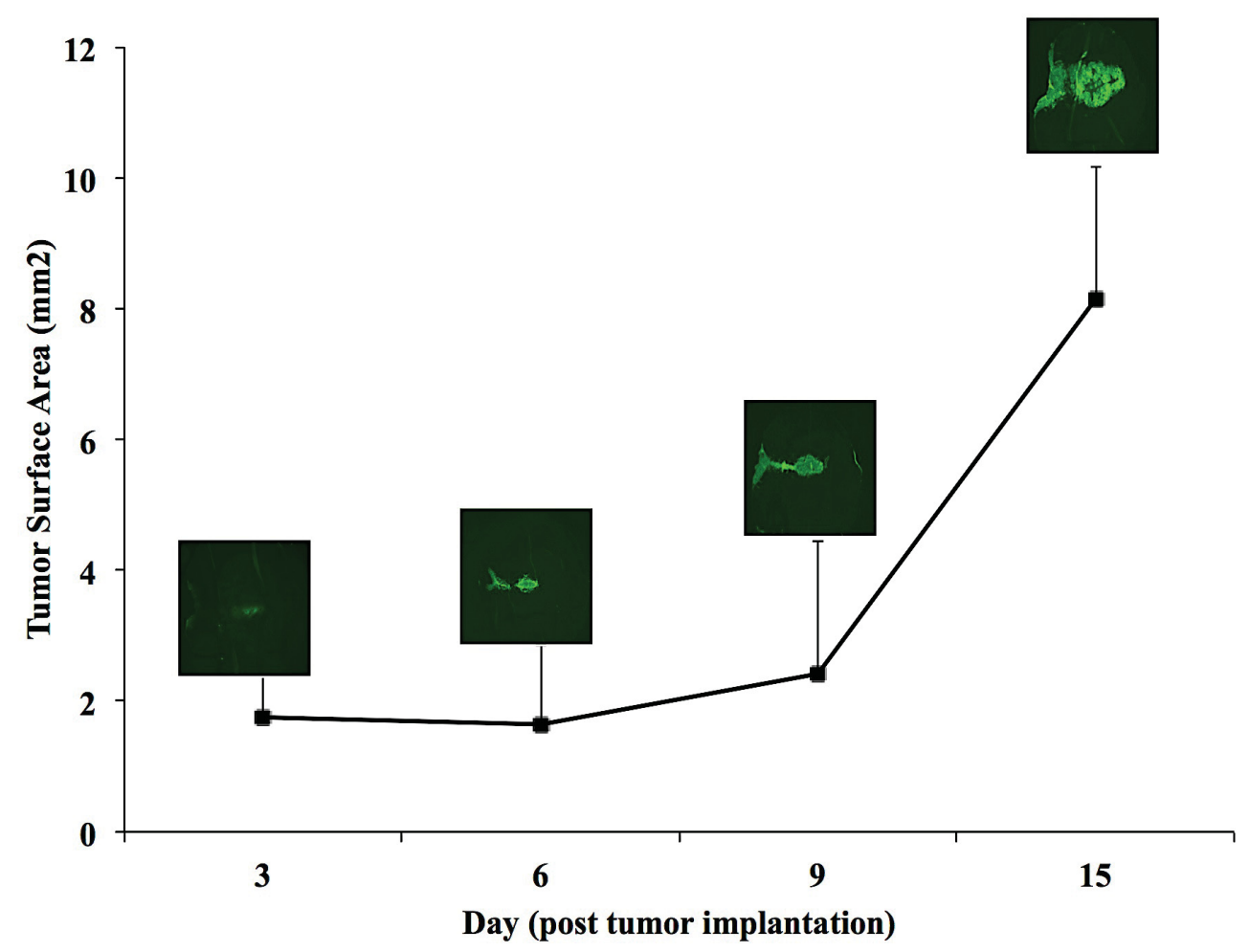

B

C
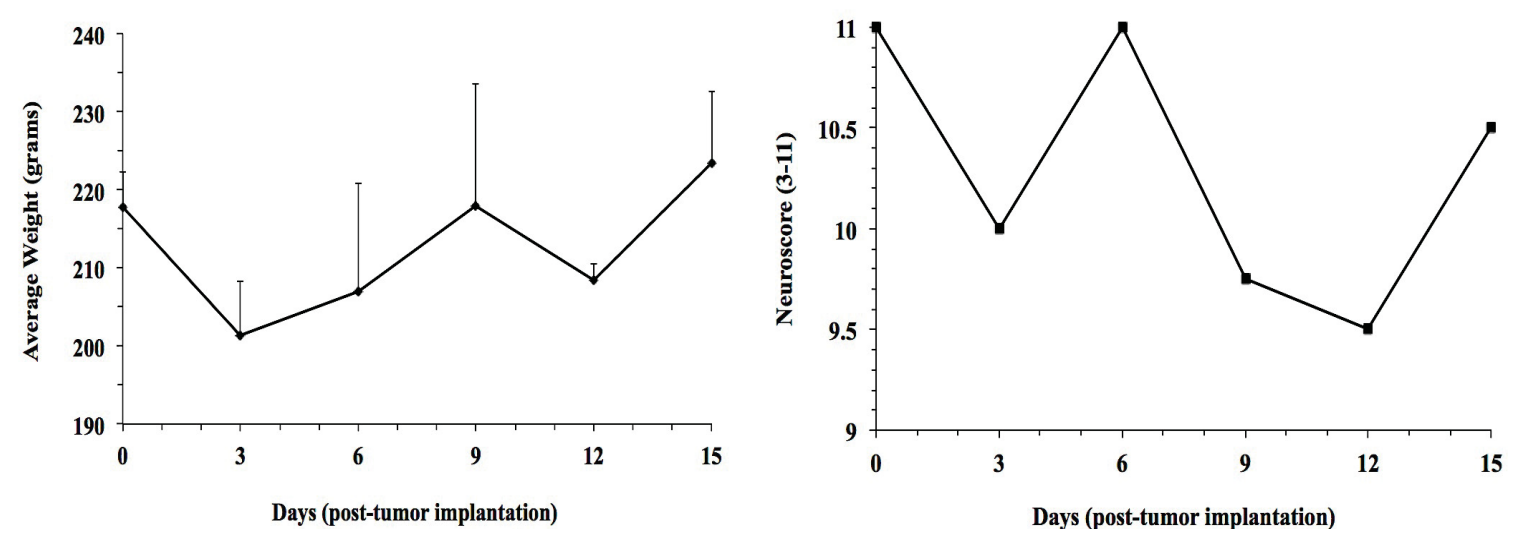

Figure 3-9. Tumor progression of F98-GFP glioma in Fischer rat over time (A) Fischer rat were implanted with 100,000 cells resuspended in $20 \mu \mathrm{L}$ of PBS on day zero. Animals were sacrificed every three days for 15 days in order to determine tumor load by GFP expressing tumor cells (See inset fluorescence micrographs). (B) Weight trends and $(C)$ Neuroscores measured every three days before euthanasia $(n=3)$. 
studies. As described in Chapter 2, F98-GFP gliomas were implanted in six week old male Fischer rat and allowed to grow for three days at which point one intratumoral injection of $10^{9}$ pfu rNCP12.1-GFP was administered (Figure 3-10).

At 15 days following tumor implantation, the first group of rNCP12.1 treated animals $(n=9)$ was sacrificed and brains were harvested and assessed for tumor size. We found that at 15 days post infection, tumor size was $66 \%$ smaller $(p<0.0013)$ than vehicle-treated controls (Figure 3-11A). Representative fluorescence micrographs are seen in Figure 3-11Bi, iii). Morbidity at time of sacrifice was minimal as determined by weight trends (Figure 3-12A) and neuroscore (Figure 3-12C). Weight trends following tumor implantation and up to one day post-treatment were as expected based on similar findings observed during the previous dose escalation study in Wistar rats. By 48 hours post-infection, weight trends in vehicle treated animals continued to increase and on average animals weighed approximately $20 \mathrm{~g}$ more than starting weights (day 0 ). On the other hand, virally treated animals continued to decline in weight up to 4 days postinfection at which point weights plateaued and slightly increased at several time points until the end of the study. At the most, weight of virally treated animals decreased $15.5 \%$ (day 11) of starting weight but never exceeded a $20 \%$ decline. Neuroscores for both vehicle only and rNCP12.1 treated animals ranged between 10 and 11 with the most common decrease in score attributed to changes in spontaneous movement.

Another group of treated animals $(n=9)$ were used to determine survival. These animals were allowed to live until criteria for euthanasia were met based on effects of viral treatment, weight trends, and/or tumor load. Based on log rank analysis of the Kaplan-Meier survival curve comparing vehicle treated to rNCP12.1 treated animals, there was a small yet significant difference in survival in animals receiving virus $(p=0.0063)$ (Figure 3-12E). Median survival of virus-treated animals was 29 days compared to 23 days in those treated with vehicle. Despite the remarkable decrease in tumor size due to rNCP12.1 treatment observed at 15 days post tumor implantation, at the time of sacrifice, tumors were not significantly different than vehicle-treated animals, displaying only an 11\% decrease in tumor load (Figure 3-11A and Bii, iv).

Immunohistochemical analysis of cryosections from an animal in the acute group was performed. H\&E stain shows areas of hypercellularity marked by necrotic tissue with infiltrating blood cells possibly marking areas where tumor became hemorrhagic

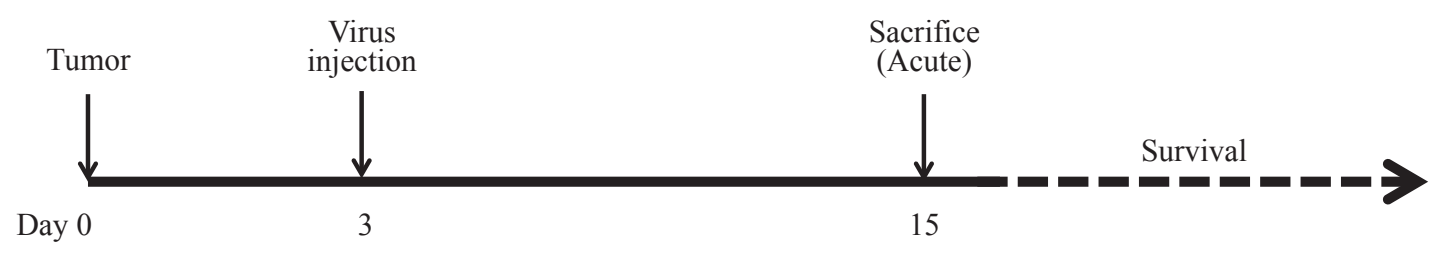

Figure 3-10. Timeline for the single rNCP12.1- GFP injection study in Fischer rat 

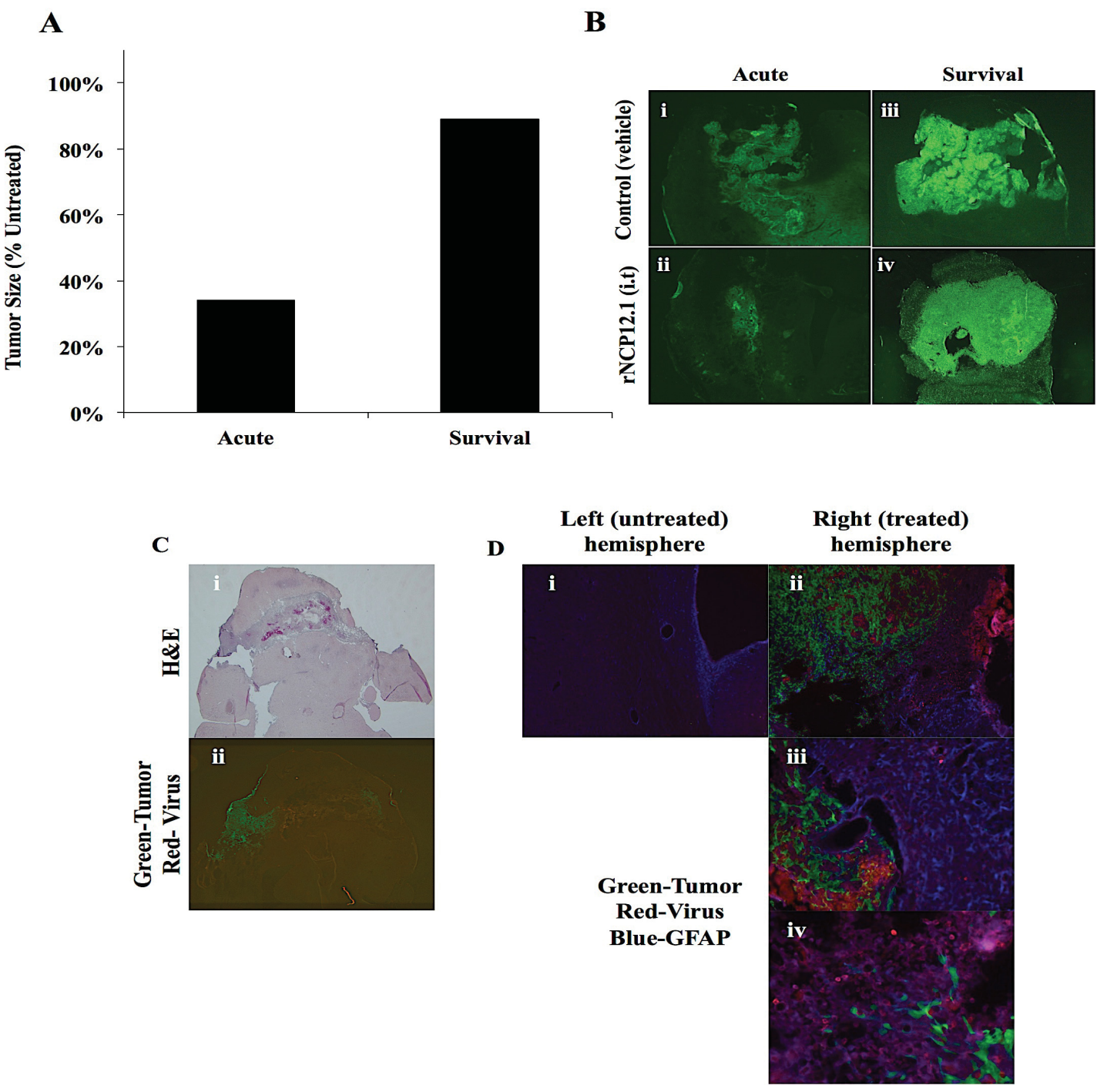

Figure 3-11. Comparisons of tumor properties in animals treated with a single i.t injection of rNCP12.1-GFP

Comparison of tumor load as a percentage of tumor load in uninfected animals in animals from the (A) acute arm of study sacrificed at 15 days following tumor implantation; $(p=0.0013)$ and survival arm, sacrificed when criteria for euthanasia were met $(p=0.2829$, ns). (B) Representative fluorescence micrographs demonstrating tumor size in (i) acute control animals (ii) acute virus-treated animals (iii) survival control animals (iv) survival virus-treated animals. (C) Micrographs of brain tissue from a treated animal in the acute arm, demonstrating patterns of inflammation and of viral infection relative to tumor. (i) H\&E stain (ii) Fluorescence; F98-GFP tumor cells (green); Virus (Red). (D) Additional labeled tissue samples from the same animal illustrating tumor (green), virus (red), and GFAP astrocytic marker (Blue) (i) left (untreated) hemisphere (ii) 10X magnification of right (treated) hemisphere, (iii) 20X magnification (iv) 40X magnification. 

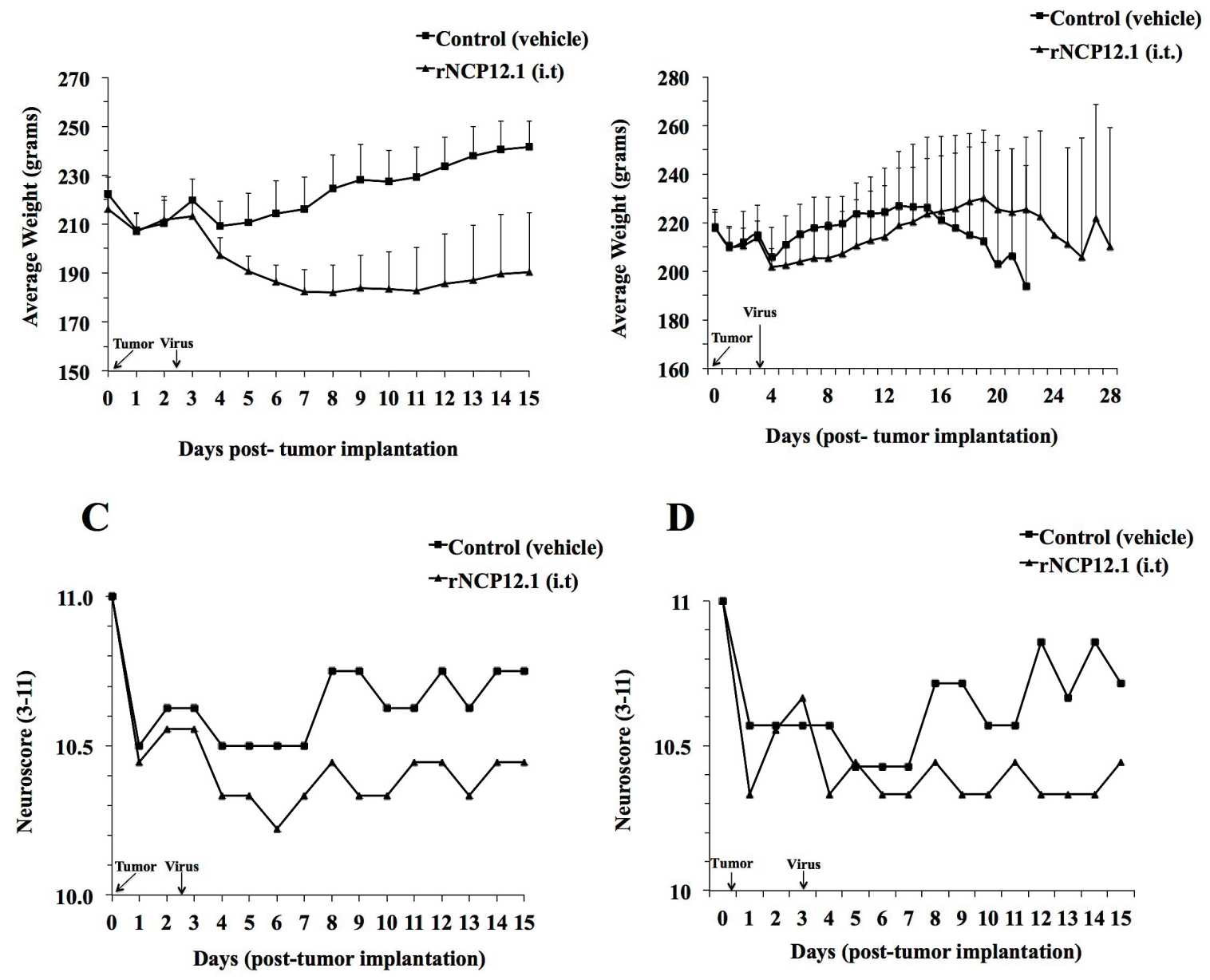

D
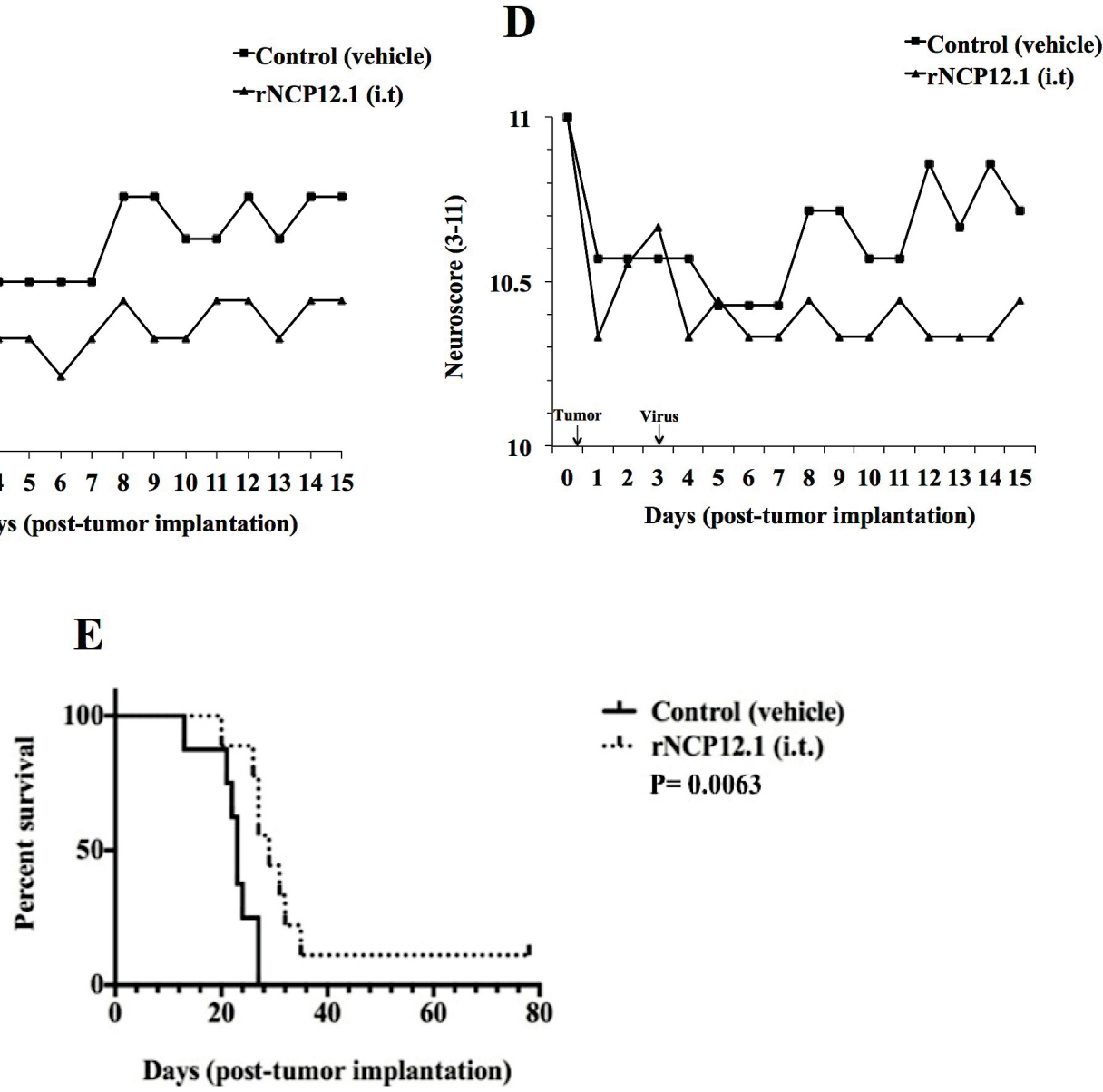

Figure 3-12. Measures of morbidity of animals treated with a single i.t. injection of rNCP12.1-GFP

Weight trends of animals in the (A) acute and (B) survival arms. Neuroscores of animals in (C) acute and (D) survival arms. (E) Kaplan-Meier survival analysis demonstrating a significant difference in survival between virus-treated animals and controls. Statistical data performed using Log-rank (Mantel-Cox) test; $\mathrm{P}=0.0063$. 
due to leaky tumor associated blood vessels (Figure 3-11Ci). In correlation, staining of virus for VSV-N using a rhodamine-conjugated antibody demonstrated areas of increased GFP expression near the surface of the brain with positive rhodamine (red) labeling intraparenchymally (Figure 3-11Cii). Additional tissue was double labeled for detection of the astrocytic marker, GFAP (glial fibrillary acidic protein) (blue) and virus (red). The uninfected hemisphere is shown for comparison and demonstrates the normal presence of GFAP astrocytic marker in the area of the subventricular zone (Figure 3-11Di). Staining of tissue in the right hemisphere reveals GFP expressing tumor with GFAP present within the tumor as well as surrounding normal tissue. Additionally, we see virus present specifically within the tumor mass (Figure 3-11Dii, iv) with some evidence of viral presence in the immediate surrounding normal tissue (Figure 3-11Div).

Morbidity based on weight trends (Figure 3-12B) and neuroscores (Figure 3-12D) were also followed in the survival arm. Weights in the survival study were followed for all animals throughout the study but only weights up to medium survival for both groups were plotted. Trends following tumor implantation and up to one day, post treatment, were consistent with the previously described studies. Unlike in the acute study, weights in survival study animals began increasing by day 2 post-treatment and at day 16 , weights of virally treated animals had surpassed those of control animals. They continued to increase for several more days while weights of control animals decreased until animals were sacrificed. Though delayed, weight trends of virally treated animals eventually decreased by day 19 and continued declining until animals were euthanized. Although weights never declined $>20 \%$ of starting weight in either group, the decreasing trend correlated with progression to death in each group. Neuroscores remained between 10-11 for all animals in both groups up to day 15. Scores were not recorded following this time point.

\section{One Intracranial Dose of rNCP12.1 Induces Both Innate and Adaptive Immune Responses in F98 Glioma Bearing Rats by 15 Days Post-Infection}

As discussed, immune responses play a very important role in the success of viral therapies. A balance between protecting the host from adverse viral effects and allowing optimal viral replication in order to kill tumor and elicit long term anti-tumor responses must exist. To this end, the immune system is often referred to as a double-edged sword in that oncolytic potential is maximized by both inhibition and promotion of various features of immunity. For example, previous studies showed that promoting IFN responses aids in protection of normal cells [289, 344], yet blocking IFN responses through the use of IFN pathway inhibitors such as rapamycin, can prolong viral activity allowing a longer therapeutic window [193]. The same is said for adaptive immune responses, particularly neutralizing antibodies that help clear virus for protection of the infected host while limiting the duration of antitumor activity.

In the present studies, we evaluated serum collected by transcardiac method from animals treated with $1 \times 10^{9}$ pfu rNCP12.1 in the acute study at the time of sacrifice. These results were also compared to sera from F98 tumor bearing animals of a subsequent study 
in which a single intracranial injection of a slightly higher dose $\left(2 \times 10^{9} \mathrm{pfu}\right)$ of $\mathrm{rNCP} 12.1$ was administered. Innate (IFN; Figure 3-13A) activity was determined using the described IFN bioassay. An adaptive (anti-rNCP12.1 antibodies; Figure 3-13B and C) response was determined by the presence of circulating antibodies using Western blot analysis and a neutralizing antibody assay. Antibodies were detected in the sera and were mostly directed against VSV glyocoprotein (G) as well as matrix (M), which are the two major antigens that give antibody responses during VSV infection. The anti-G response produces neutralizing antibody and is protective against infection in animals. Some anti$\mathrm{N}$ antibodies were observed but with a lower signal than those directed against the other viral antigens. Anti-rNCP12.1 neutralizing antibodies (nAbs) were detected using a neutralizing assay in which sera of infected animals was treated at $56^{\circ} \mathrm{C}$ for 35 minutes in order to inactivate any residual virus [344]. Serial dilutions (1:2 to 1:1000) of heatinactivated sera was combined with 200 pfu of wtVSV inoculum and incubated together for 45 minutes at $37^{\circ} \mathrm{C}$ to allow potential binding of antibody and viral antigen.

Serum/inoculum mix was then applied to BHK cells to determine the dilution at which $50 \%$ inhibition of CPE was observed relative to fetal bovine serum/inoculum (negative control). Neutralization is plotted as the inverse of the dilution required for $50 \%$ inhibition of infection at 24 hours post-treatment. The positive control is a polyclonal antibody developed in rabbits following immunization with intact UV- inactivated virus (TN-1). It is the antibody used by our lab for neutralization experiments and in the detection of VSV proteins for other molecular techniques. The dilution of TN-1 needed for $50 \%$ inhibition was 1:800 therefore the titer plotted in the neutralization assay is 800 .

Similar to in vitro studies, rNCP12.1 induces systemic production of detectable IFN in vivo. Regardless of dose, IFN activity ranged from $2-4 \times 10^{2} \mathrm{U} / \mathrm{mL}$. One of the 4 animals in the lower dose group displayed $\sim 10$ fold lower IFN activity than other animals. When compared to the nAb detection assay, nAb against rNCP12.1 were consistently present in almost all treated animals with titers ranging between 600-1000, with some animals having even greater neutralizing activity than the positive control. Not surprisingly, the one animal with the least detected IFN activity also expressed the lowest titer of anti-rNCP12.1 nAb signifying a possible error in delivery of initial dose of virus. All sera was evaluated for presence of virus using our standard plaque assay in BHKs and no infectious virus was detected in any samples (data not shown).

From these results, we conclude that in the first 2 weeks of treatment, an obvious decrease in tumor load is experienced in animals receiving one intratumoral dose of $10^{9}$ pfu rNCP12.1. These results were accompanied by low morbidity as seen by weight trends and neurological scoring as well as induction of both peripheral innate (IFN) and adaptive $(\mathrm{nAb})$ immune responses. Nonetheless, reduction in tumor load was not sustainable though it did result in a significant yet minor prolongation in survival of treated animals. Gliomas eventually recurred at an accelerated rate with tumors of virustreated animals being comparable in size to those treated with vehicle only. 
Figure 3-13. Detection of immune responses at day 15 in animals treated with a single injection of rNCP12.1

(A) IFN activity as measured by the IFN bioassay (units (U)/ $\mathrm{mL}$ ). Detection of antiVSV antibodies in sera of treated animals by (B) Western Blot analysis confirming presence of circulating antibodies directed against VSV antigens. Serum was diluted to 1:100 for each tested sample. R6F, diluted at 1:5000, was used as the positive control. It is a polyclonal antibody developed in rabbits immunized with detergent disrupted wtVSV. Negative (untreated) control was serum from tumor-bearing animals not treated with virus. (C) Detection of anti-VSV neutralizing antibodies $(\mathrm{nAb})$ in sera of treated animals using a neutralizing assay. Neutralizing titer was determined as the dilution of sample needed for $50 \%$ inhibition of wtVSV. TN-1, a polyclonal antibody developed in rabbits immunized with intact UV inactivated virus, served as the positive control. TN-1 requires at least a dilution of 1:800 for 50\% inhibition of wtVSV induced CPE therefore the $\mathrm{nAb}$ titer is 800 

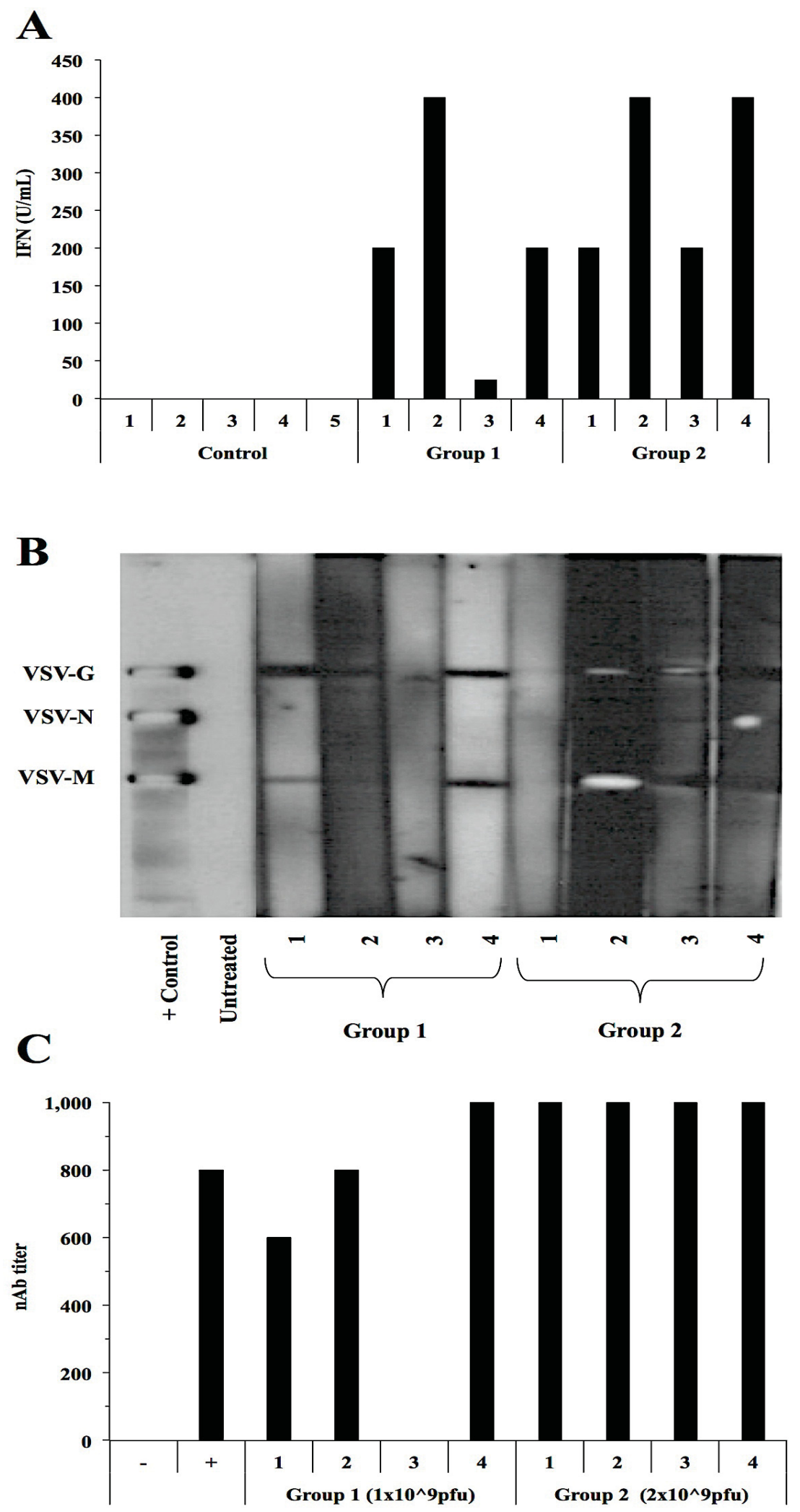


\section{Evaluation of Various Methods of Viral Administration on Safety and Efficacy of rNCP12.1 as an Oncolytic Treatment for Intracranial Glioma}

As demonstrated, components of both innate and adaptive immunity are elicited in response to rNCP12.1 infection in vivo. Due to this phenomenon, a number of delivery methods have been proposed as potential ways of enhancing effectiveness of viral therapy. These methods are based on their ability to either shield virus from the immune system or modulate the immune system by suppressing certain aspects of immunity long enough to achieve optimal oncolysis. Other methods consider the role of the tumor microenvironment in hindering spread of virus. This includes, but is not limited to, increases in pressure caused by the tumor mass and associated inflammation that can prevent infusion of virus within the tumor as well as sequestration of virus by components of the extracellular matrix [178-181]. In the following studies, we have tested a number of delivery methods and their effects on efficacy of treatment and safety as seen through weight trends, neuroscore, and overall survival, taking into account the role of both tumor microenvironment as well as components of the immune system.

\section{Constant Infusion of rNCP12.1 over Time by Infusion Pumps Is Not a More Effective Method of Viral Delivery}

Taking into account the effects of the microenvironment on the oncolytic efficacy of viral therapy, we tested the use of subcutaneously implanted micro-osmotic pumps (Alzet Model 1003D) in their ability to reduce tumor by infusing a small yet constant dose of virus over time. These implantable pumps are loaded with a pre-determined concentrated volume of therapy and implanted subcutaneously where they deliver a continuous flow ( $1 \mu \mathrm{L} /$ hour) of therapeutic agent over a limited time frame (three days). Considering our therapeutic agent of choice is a virus with a potency that is sensitive to temperature and time, we first performed a stability study in which we tested changes in initial titers of virus over 3 days at $37^{\circ} \mathrm{C}$. Alzet pumps containing $5 \times 10^{10} \mathrm{pfu} / \mathrm{mL}$ rNCP12.1 were incubated at $37^{\circ} \mathrm{C}$ for three days. Viral titers were tested at $\mathrm{t}=0,4,8$, and 24 hours then every 24 hours for the next 2 days using our standard plaque assay in BHK cells. We found that viral activity decreased approximately 10 fold every 24 hours for the first two days eventually dropping to no infectious virus by three days under the specified conditions (Figure 3-14). Though this temperature is an estimate of body temperature in the animal model and is likely lower given the superficial implantation site of the pumps, these results provided an idea of changes in virus concentration that should be expected during the study. We therefore assume that our delivered virus by the end of the first day of infusion would be approximately 10 fold lower than the loading dose with day two titers being 100 fold lower. In addition, it is likely that no infectious virus would be infused by the end of day three.

Next, we evaluated this delivery system using our oncolytic virus in an immunocompetent F98 glioma model. Fischer rats were implanted with F98 glioma cells on day zero as described. At five days post-implantation, animals were given a single dose of rNCP12.1 by intratumoral injection. On the same day as injection, Alzet pumps 


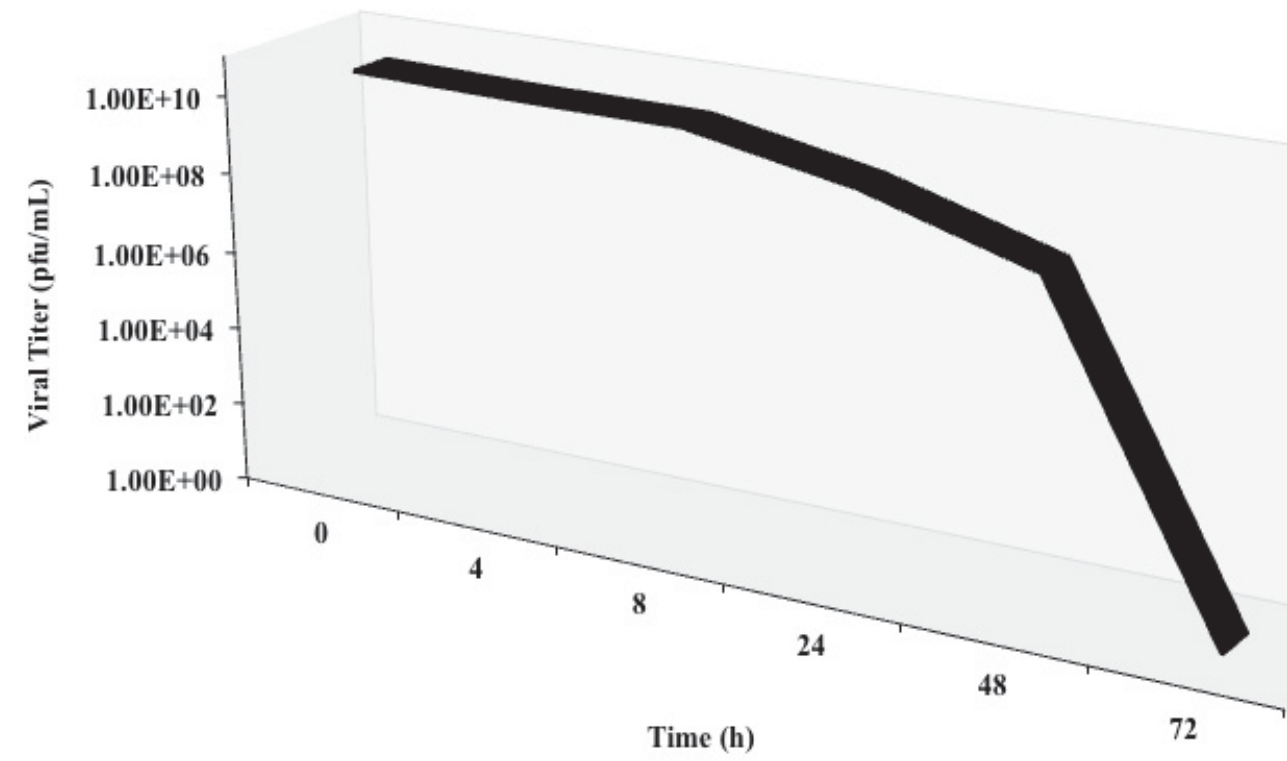

Figure 3-14. Stability of rNCP12.1 during the use of Alzet infusion pumps A starting titer of $5 \times 10^{10} \mathrm{pfu}$ of rNCP12.1 was added to Alzet pumps then incubated for three days at $37^{\circ} \mathrm{C}$. Titers were performed at $\mathrm{t}=0,4,8,24,48,72$ hours in order to determine viral stability over time. Titers decreased 10 fold every day for two days with no infectious virus detected at the end of the study 
containing varying doses of rNCP12.1 were secured subcutaneously and allowed to infuse virus at a rate of $1 \mu \mathrm{L} /$ hour $(24 \mu \mathrm{L} /$ day) from days 5-8 (Table 3-2; Figure 3-15A). Animals were followed carefully with weights and neuroscore checked every three days until criteria for euthanasia were met.

We observed a non-significant ( $\mathrm{p}=0.5932$ ) $27 \%$ decrease in tumor size of animals receiving the medium dose of virus versus $18 \%$ decrease in low dose and $4 \%$ decrease in high dose treated animals as compared to control animals (no vehicle). Interestingly, animals receiving vehicle only displayed the highest decrease in tumor load with tumors reduced by $34 \%$ by the end of the study (Figure 3-16A). Representative micrographs of tumors are shown in Figure 3-16B. Weight trends (Figure 3-16C) and neuroscores (Figure 3-16D) were measured every three days with more frequent checks if additional signs of distress were observed.

Specifically, weight trends compared to the previous study were much more affected. Control animals not receiving any vehicle demonstrated expected trends in weight with a slight fall after tumor implantation followed by a rise and then subsequent fall as animals started to experience effects of tumor burden. Animals receiving vehicle only displayed similar trends in weight as control animals, though at an approximately 15 grams lower weight at each time point. Virus treated animals all displayed a continuous decline in weight following therapy until time of sacrifice. Animals receiving low dose lost less weight than animals in the other two groups with weight at time of sacrifice approximately $27 \%$ lower than starting weight. Neuroscores using the RCS on average were lower compared to scores of animals that received only one intratumoral injection of rNCP12.1. Animals in the high dose group surprisingly maintained a neuroscore between 10-11 whereas scores averaged approximately 9-10 by the end of the study for all groups including control animals. Low and medium dosed animals experienced the most neurological decline with neuroscores ranging from 9.0 to 9.5 during the last few days of life. As we saw with a single injection, tumors were not significantly different between treated and untreated animals however unlike animals that received one injection of virus, survival was not significantly different between the treated groups nor were they significantly different from control (Figure 3-16E). The only significant difference in survival was observed by comparing survival of control animals that received vehicle and animals receiving the lowest dose of rNCP12.1 (Figure 3-16F). Median survival was 21 days and 27 days, respectively. Though animals receiving vehicle only had the smallest tumors on average, they also had the lowest overall survival (Table 3-3).

In summary, continuous infusion after initial injection of rNCP12.1 did not enhance anti-tumor activity or survival. There was no significant difference in tumor size based on dosing of initial injection or infusion. Survival was not enhanced compared to control animals. However it appears that animals receiving vehicle had an overall lower survival that was significantly different from animals treated with low dose virus. The infusion method appeared to have a negative effect on morbidity and animals in the study had an overall lower average weight and neuroscore than observed in previous studies not using this method. In conclusion, this method of delivery was not effective at enhancing tumor reduction and in improving morbidity in treated animals. 
Table 3-2. Description of treatment groups in Alzet pump study

\begin{tabular}{lcc}
\hline \multicolumn{1}{c}{ Treatment Group } & Injection Dose & $\begin{array}{c}\text { Overall Pump Infusion } \\
\text { Dose }\end{array}$ \\
\hline Low & $4 \times 10^{8} \mathrm{pfu}$ & $1.6 \times 10^{7} \mathrm{pfu}$ \\
Medium & $1 \times 10^{9} \mathrm{pfu}$ & $4 \times 10^{7} \mathrm{pfu}$ \\
High & $2.5 \times 10^{9} \mathrm{pfu}$ & $1 \times 10^{8 \mathrm{pfu}}$ \\
\hline
\end{tabular}

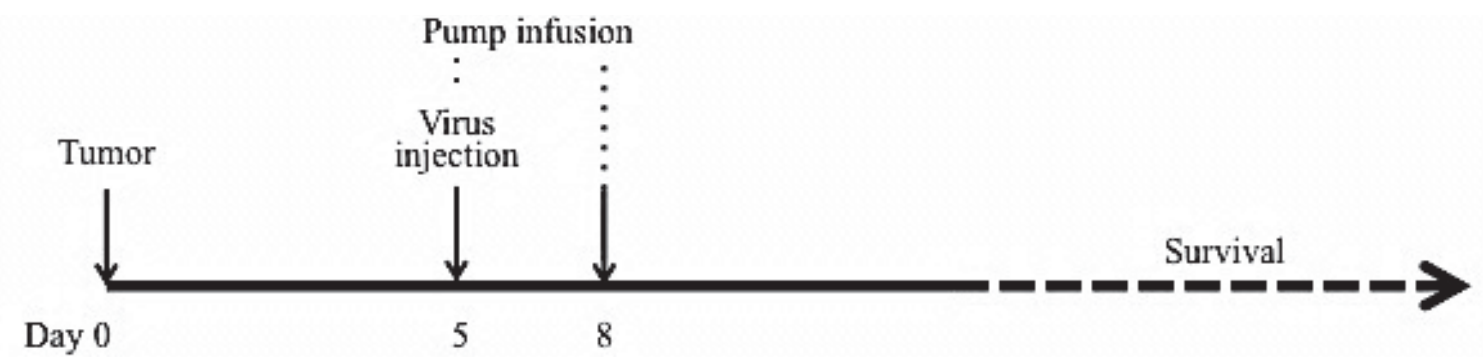

Figure 3-15. Timeline for Alzet pump delivery study 
Figure 3-16. Comparison of tumor load in animals treated with varying doses of rNCP12.1 delivered by Alzet pump infusion

At day five, post-tumor implantation, animals were given one i.t. injection of rNCP12.1 at varying doses per group. On the same day, Alzet pumps were implanted and infusion of virus occurred over three days. Animals were sacrificed when criteria for euthanasia were met. (A) There was no significant difference in size of tumors between treated and untreated $(p=0.5932)$. Level of significance determined by One Way ANOVA using mean values of exact tumor size $\left(\mathrm{mm}^{2}\right)$. (B) Representative fluorescence micrographs of each group demonstrating GFP expressing tumor. Morbidity of animals as determined by (C) Weight trends and (D) Neuroscore. (E) Kaplan-Meier survival analysis demonstrating a significant difference in survival. Statistical data performed using Logrank (Mantel-Cox) test; $\mathrm{P}=0.0140$. (F) Kaplan-Meier survival analysis demonstrating a significant difference in survival between Control (vehicle) animals and Low Dose animals; $\mathrm{P}=0.0073$. Statistical data performed using Log-rank (Mantel-Cox) test 

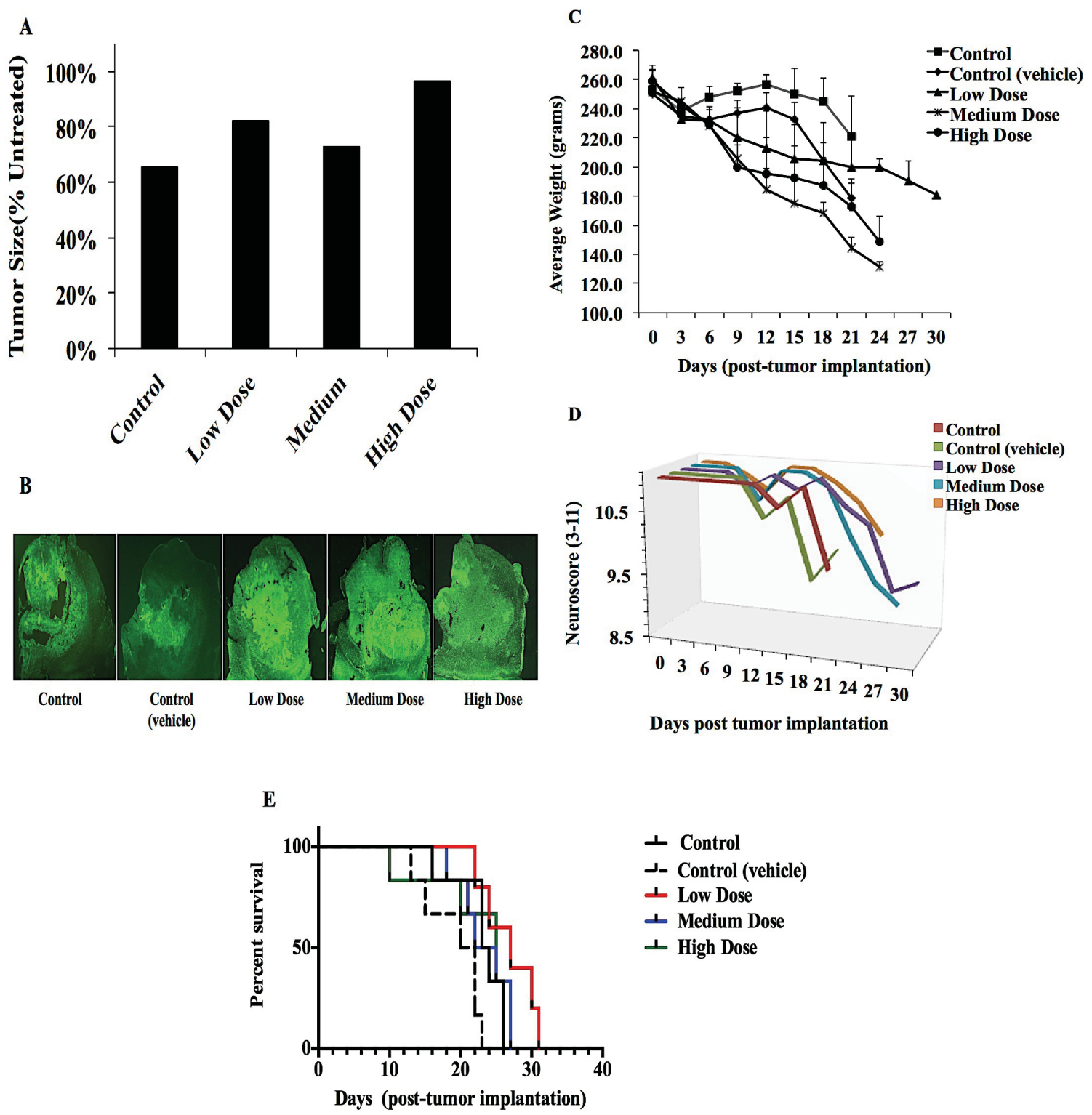

$\perp$ Control

\lrcorner - Control (vehicle)

$\perp$ Low Dose

$\perp$ Medium Dose

$\perp$ High Dose

F

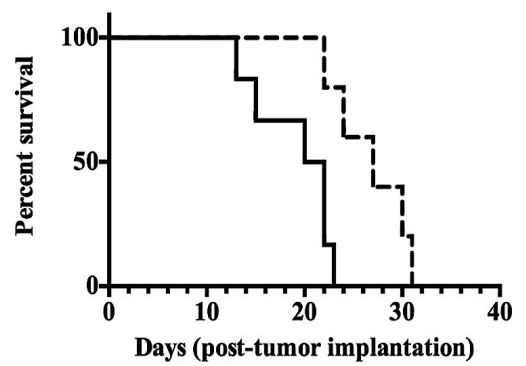

$\perp$ Control (vehicle)

- Low Dose 
Table 3-3. Summary of survival for animals treated in Alzet pump study relative to vehicle control

\begin{tabular}{lcc}
\hline \multicolumn{1}{c}{ Treatment Group* } & Median Survival (Days) & $\begin{array}{c}\text { Significant } \\
\text { Survival } \\
\text { (vs Control-Vehicle) }\end{array}$ \\
\hline Control & 23.5 & No \\
Control (Vehicle) & 21 & - \\
Low & 27 & Yes \\
Medium & 23.5 & No \\
High & 25 & No \\
\hline
\end{tabular}

*Control-tumors implanted on day 0 with no further surgeries; Vehicle Controlimplanted tumors on day 0 with injection of viral vehicle only (TN-sucrose) on day 5 


\section{Switching Serotype Enhances Survival and Prolongs Tumor Reduction in F98 Glioma Bearing Animals}

In an effort to overcome the effects of the adaptive immune system, specifically of neutralizing antibodies, on viral therapy, we performed a study in which we administered two separate doses of rNCP12.1, intratumorally. As previously discussed, VSV has two major serotypes, New Jersey and Indiana. The glycoprotein of these serotypes possess only 50\% amino acid sequence homology therefore infection of one serotype should not protect against subsequent infection with the other serotype. For the initial dose, all treated animals received $\mathrm{rNCP} 12.1$, Indiana serotype $\mathrm{G}$ protein $\left(\mathrm{G}_{\mathrm{I}}\right)$. For the second dose, animals received either the same $\mathrm{rNCP}^{2} .1\left(\mathrm{G}_{\mathrm{I}}\right)$ or $\mathrm{rNCP} 12.1$ having a $\mathrm{G}$ protein from the New Jersey serotype $\left(\mathrm{G}_{\mathrm{NJ}}\right)$. Hence, group Ind/Ind received two injections of the same serotype and Group Ind/NJ received the New Jersey serotype for the second injection. The timeline of the study is depicted in Figure 3-17 with a description of dosing in Table 3-4. There were two separate groups based on time of sacrifice, an acute study to determine tumor load at 15 days post-tumor implantation and a second group sacrificed when euthanasia criteria were met. At 15 days post-tumor implantation, tumors from animals in both treated groups were found to be $63-65 \%$ smaller than tumors from animals receiving vehicle only $(p=0.0149)$ (Figure 3-18A). Representative tumor images are shown in Figure 3-18B-G. Morbidity as determined by weight trends (Figure 3-19A) and neuroscores (Figure 3-19B) were assessed throughout the 15 days. Animals receiving treatment regardless of serotype experienced near identical weight trends with the largest decrease in weight of approximately $30 \%$ experienced at 12 days post-tumor implantation followed by a $25 \%$ increase in weight by day 15. Control animals displayed a gradual decline with weights at sacrifice approximately $15 \%$ lower than starting weight. All animals maintained a neuroscore of 11 in the first 15 days (data not shown). Past 15 days post-implantation, tumors were reduced in size though non-significantly (Figure 3-18A). Of note, animals in the Ind/NJ treatment arm had 54\% smaller tumors than control animals whereas, tumors in the Ind/Ind treatment arm were 19\% reduced. Weights were more varied later in the study with Ind/Ind animals weighing $\sim 15-25$ grams more than animals in the Ind/NJ group (Figure 3-19B). The largest decrease in weight of $22 \%$ of the starting weight in the Ind/NJ group was observed at day 15, post-tumor implantation, and the largest decrease of $13.4 \%$ in the Ind/Ind group observed at day 12 . Neuroscores were again maintained at 11 during the first 15 days with scores dropping as low as 7 and 8 by the time of sacrifice in Ind/Ind treated animals and Ind/NJ treated animals respectively (Figure 3-19C). For both treatment groups, there was an identical and significant increase in survival with median survival of 24 days compared to median survival of 20 days in control animals (Figure 3-19D).

From these studies we conclude that double injections of rNCP12.1 administered at a 5 day interval significantly decreased tumor load with minimal morbidity by 15 days some morbidity demonstrated by neurological deficits toward the very end of the study. 


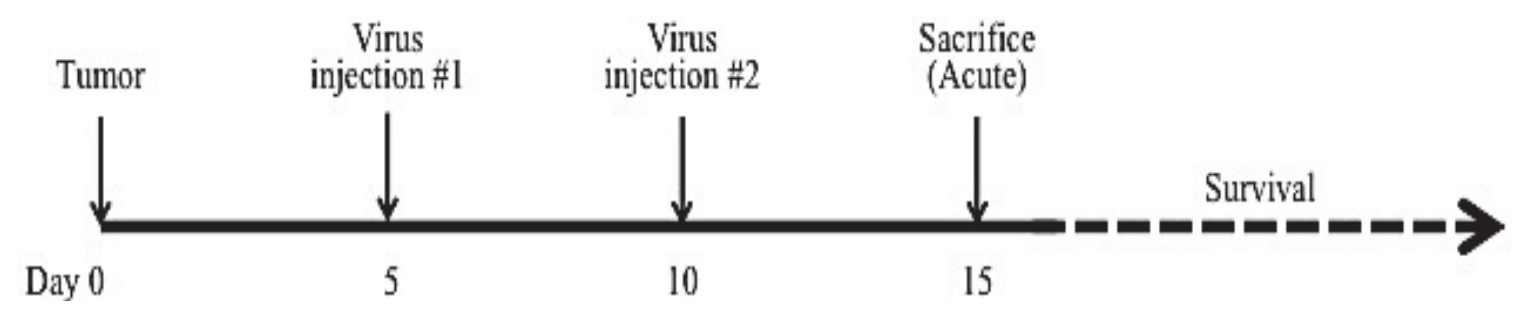

Figure 3-17. Time line of Indiana/New Jersey double injection study

Table 3-4. Description of treatment groups in Indiana/New Jersey double injection study

\begin{tabular}{lll}
\hline \multicolumn{1}{c}{ Treatment Group } & \multicolumn{1}{c}{ Serotype } & \multicolumn{1}{c}{ Dose } \\
\hline \multirow{2}{*}{ Ind/Ind } & 1. & Indiana \\
& 2. Indiana & \multirow{2}{*}{$10^{9} \mathrm{pfu}$ for ALL injections } \\
Ind/NJ & 1. Indiana & \\
& 2. New Jersey & \\
\hline
\end{tabular}


Figure 3-18. Evaluation of animals treated with Indiana/Indiana versus Indiana/New Jersey double injection

On day five, post-tumor implantation, animals were given one i.t. injection of $10^{9}$ pfu of rNCP12.1 1 -GFP. On day 10 post-tumor implantation, animals were given a second injection of a $10^{9} \mathrm{pfu}$ dose of either rNCP12.1 $1_{\mathrm{I}} \mathrm{GFP}$ or rNCP12.1 $1_{\mathrm{NJ}} \mathrm{GFP}$. Animals were either sacrificed at day 15 post-tumor implantation (acute arm) or when euthanasia criteria were met (survival arm). (A) Comparison of tumor load demonstrated significantly smaller tumors in both treated groups $(\mathrm{p}=0.0149)$ for animals sacrificed at day 15. However, there was no significant difference in the size of tumors in treated animals of the survival arm relative to control $(\mathrm{p}=0.1329)$, though tumors of animals receiving a serotype switch for the second injection did have 54\% smaller tumors.

Representative fluorescence micrographs of animals in acute arm receiving (B) no treatment, (C) two doses of Indiana serotype, or (D) one dose of Indiana serotype and one dose of New Jersey serotype and animals in the survival arm receiving (E) no treatment, (F) two doses of Indiana serotype, or $(\mathrm{G})$ one dose of Indiana serotype and one dose of New Jersey. Levels of significance were determined by One Way ANOVA using mean values of exact tumor size $\left(\mathrm{mm}^{2}\right)$. 

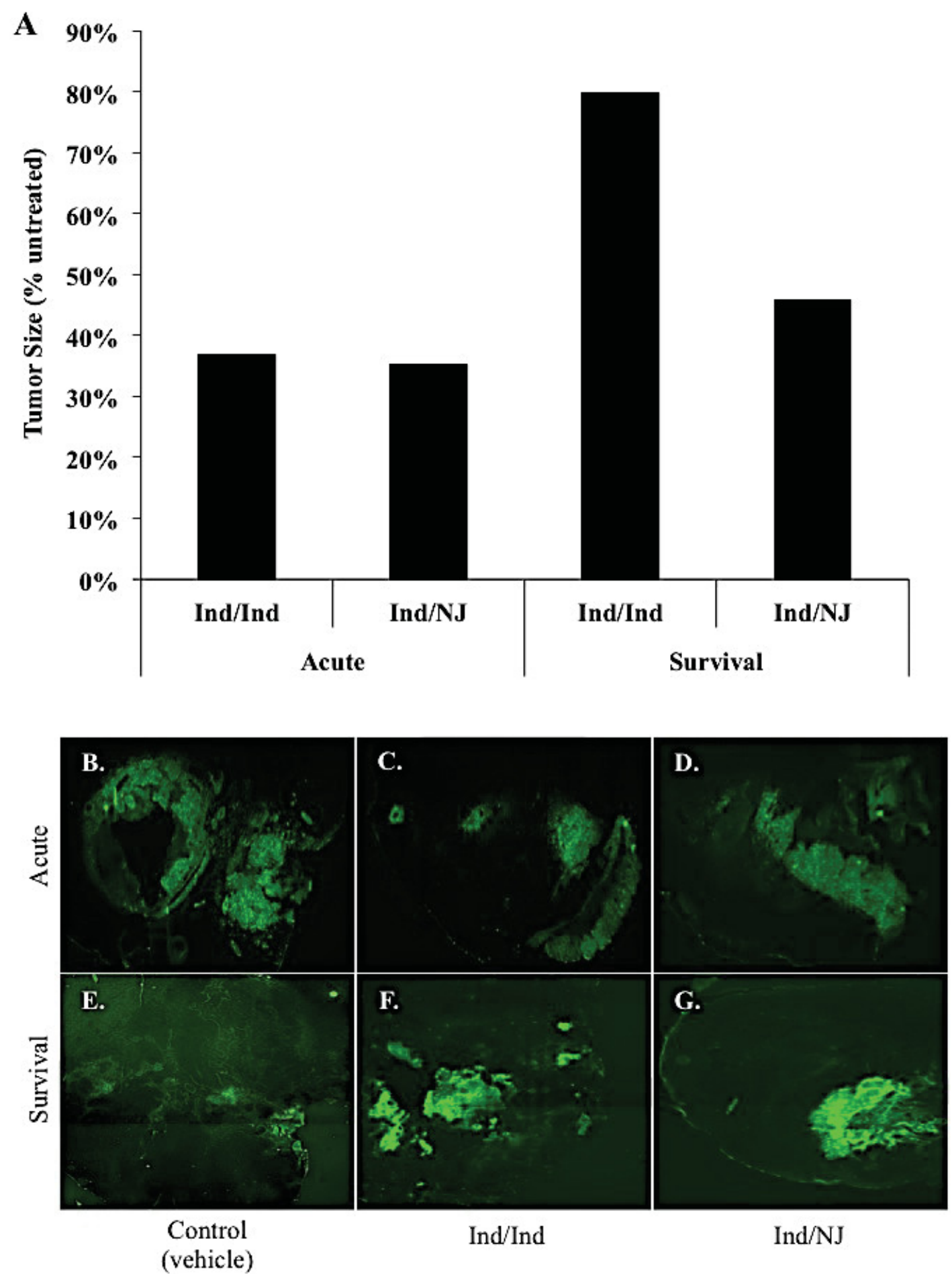

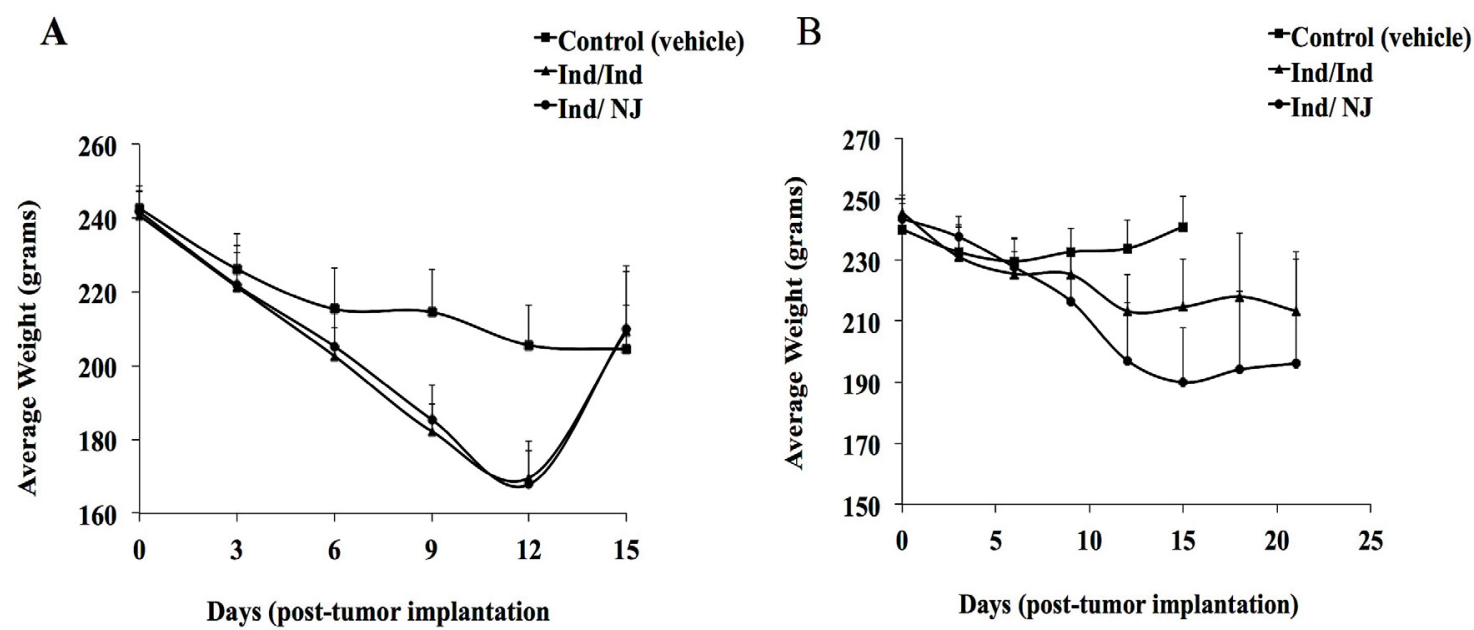

$\mathrm{C}$

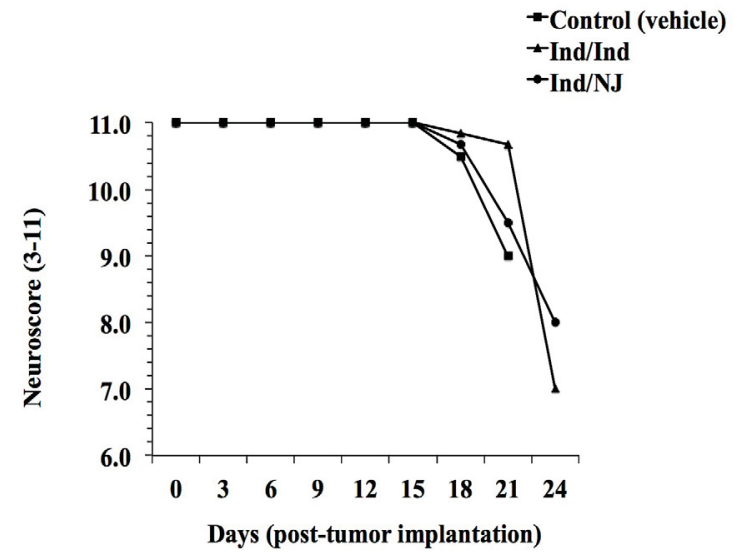

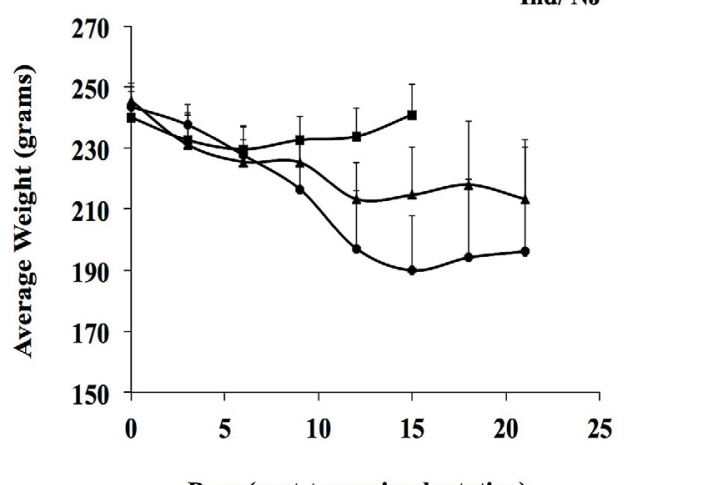

$\mathrm{D}$

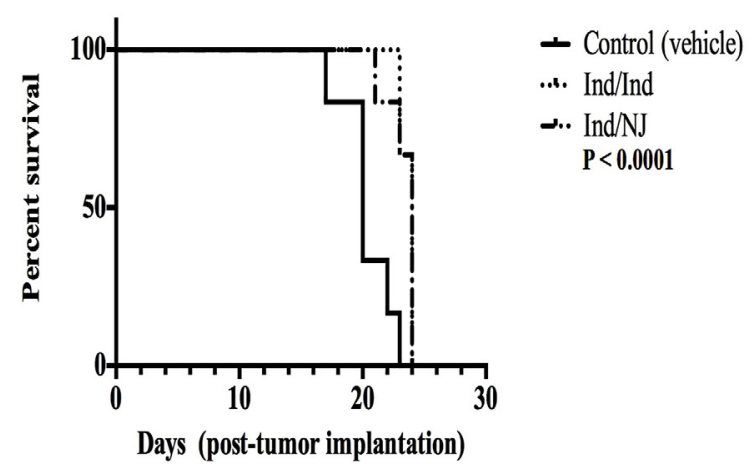

Figure 3-19. Morbidity of animals treated in the Indiana/Indiana versus Indiana/New Jersey double injection study

Morbidity of animals in acute arm as determined by (A) Weight trends. Morbidity of animals in survival arm as determined by (B) Weight trends and (C) Neuroscore (D) Kaplan-Meier analysis of survival demonstrating a significant difference in both treated groups relative to control (vehicle); $\mathrm{P}<0.0001$. Statistical data performed using Log-rank (Mantel-Cox) test. 


\section{Administration of rNCP12.1 by Tumor "Carrier Cells" as a Potential Method of Delivering Virus for the Treatment of Intracranial Glioma}

\section{Infected tumor carrier cells are unable to form intracranial glioma}

In order to determine if infected tumor cells maintained the ability to form tumors intracranially, we infected F98 glioma cells in vitro at an MOI of 0.1 or 10 . At one hour post-infection, cells were harvested and combined with uninfected cells at a ratio of 1:1 or 1:10 (infected: uninfected). Accordingly, 4 different treatment arms (Group 1-4) were prepared and tested (1. MOI of 1 at 1:1 infected: uninfected cells; 2 . MOI of 1 at 1:10 infected: uninfected cells; 3 . MOI of 10 at 1:1 infected: uninfected cells; 4. MOI of 10 at 1:10 infected: uninfected cells) (Table 3-5). On day zero, $10^{5}$ cells prepared for each treatment group were intracranially implanted in Fischer rats. Weight trends and neuroscores were assessed daily for morbidity, and at 15 days after implanting cells, animals were sacrificed and brains were harvested and processed for further analyses of tumor load and presence of virus (Figure 3-20).

We found that only implanted cells that were never infected in vitro (control) developed into visible tumors while all animals receiving pre-infected cells (PC) did not have visible tumors based on detection of GFP expressing cells (Figure 3-21A). All animals experienced a similar drop in weight, one day post tumor implantation of $\sim 10 \%$ which gradually increased in all groups except control animals and animals of the Group 1 treatment arm. Control animals continued to decline in weight until day 7 post implantation at which point weights increased until the end of the study. Group 1 animals maintained a steady weight following their initial drop on day one postimplantation (Figure 3-21B). Neuroscores were stable with an RCS of 11 throughout the study for all groups except animals in treatment Group 1 with scores averaging between 9.5-10 during the second half of the study (Figure 3-21C).

From these results, we concluded that implantation of pre-infected tumor cells lost tumorigenicity as a result of viral infection. Furthermore, following exposure to infected tumor cells, non-infected cells became infected also losing their ability to form tumors. Animals tolerated this treatment well without significant morbidity. This pilot study provided the information needed to next evaluate infected tumor cells as vehicles for oncolytic rNCP12.1 viral treatment.

\section{Pre-infected tumor cell carriers cause tumor load reduction with moderate morbidity at early time points with later time points inconclusive}

To test the efficacy and safety of using pre-infected tumor cells as carriers for virus in our glioma model, we first established F98-GFP gliomas in 6 week old male Fischer rats as described. On day four post-implantation, F98-GFP cells were infected in vitro at an MOI of 3 . At 1 hour post-infection, $10^{3}$ cells were harvested and re-suspended in $10 \mathrm{~mL}$ PBS vehicle. These cells were injected into the previous injection site used for 
Table 3-5. Description of treatment groups in evaluation of pre-infected tumor cell implantation study

\begin{tabular}{lccc}
\hline Treatment Group & MOI & $\begin{array}{c}\text { Ratio } \\
\text { Infected: Uninfected }\end{array}$ & \# of Cells Implanted \\
\hline $\mathbf{1}$ & $\mathbf{0 . 1}$ & $\mathbf{1 : 1}$ & \\
$\mathbf{2}$ & & $\mathbf{1 : 1 0}$ & $10^{5}$ cells $/ 10 \mu \mathrm{L}$ \\
$\mathbf{3}$ & $\mathbf{1 0}$ & $\mathbf{1 : 1}$ & vehicle $(\mathrm{PBS})$ \\
$\mathbf{4}$ & & $\mathbf{1 : 1 0}$ & \\
\hline
\end{tabular}

Infection and

implantation

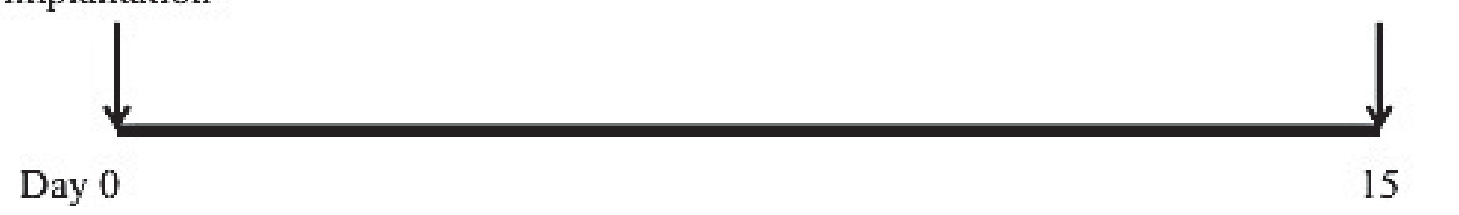

Figure 3-20. Timeline for evaluation of pre-infected tumor cell implantation study 
Figure 3-21. Evaluation of tumor implantation by rNCP12.1-GFP pre-infected F98 glioma

At day zero, F98 glioma cells were infected in vitro at an MOI of 0.1 or 10 and cells were harvested and combined with uninfected cells at a ratio of $1: 1$ or $1: 10$, infected:

uninfected. $10^{5}$ cells were prepared for each treatment group and intracranially implanted and on day 15, animals were sacrificed. (A) Comparison of tumor load. Only animals receiving vehicle only treated glioma cells (control) formed detectable tumors. Morbidity of animals as determined by (B) weight trends and (C) neuroscore. 

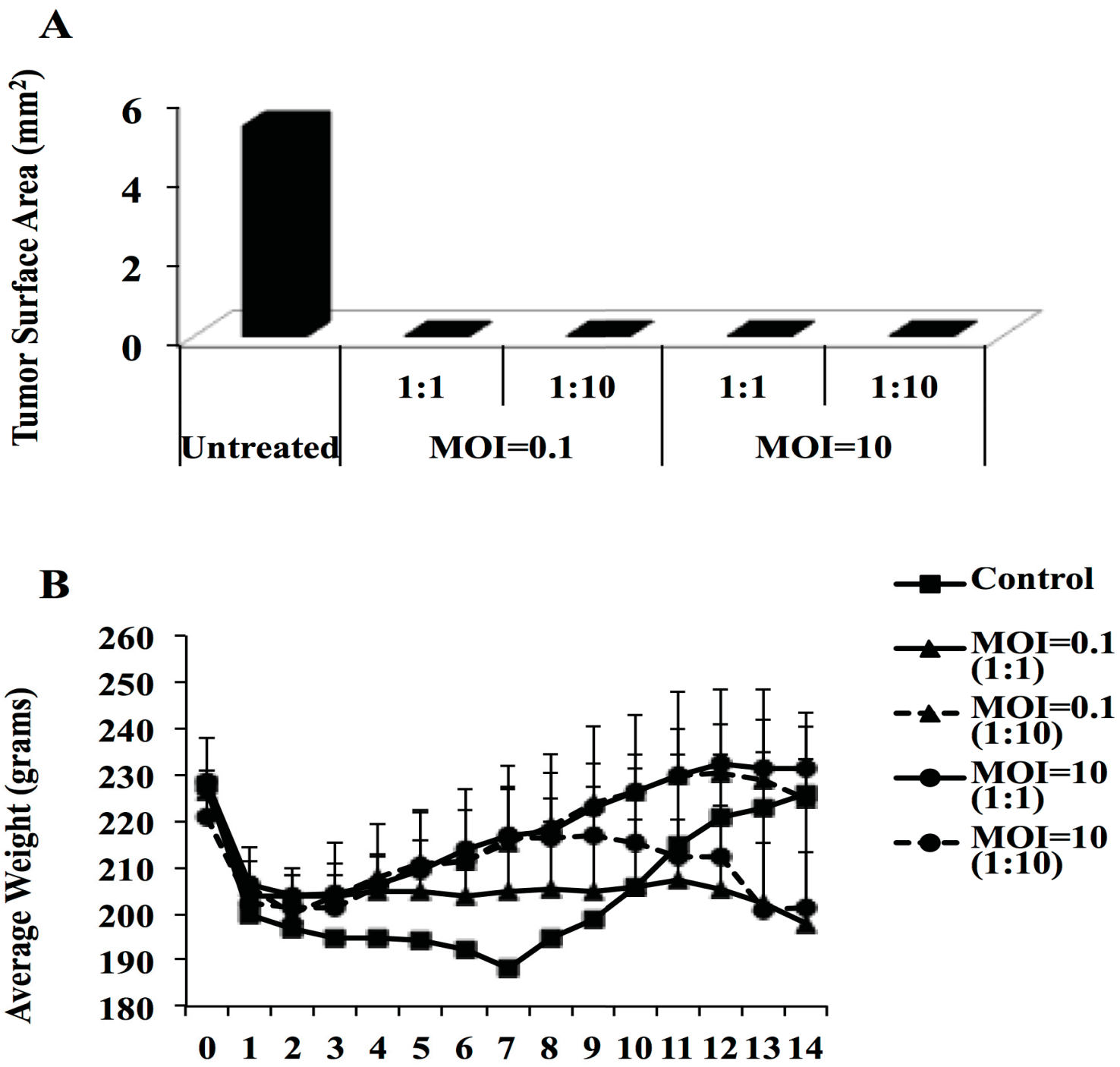

C

Days (post-tumor implantation)

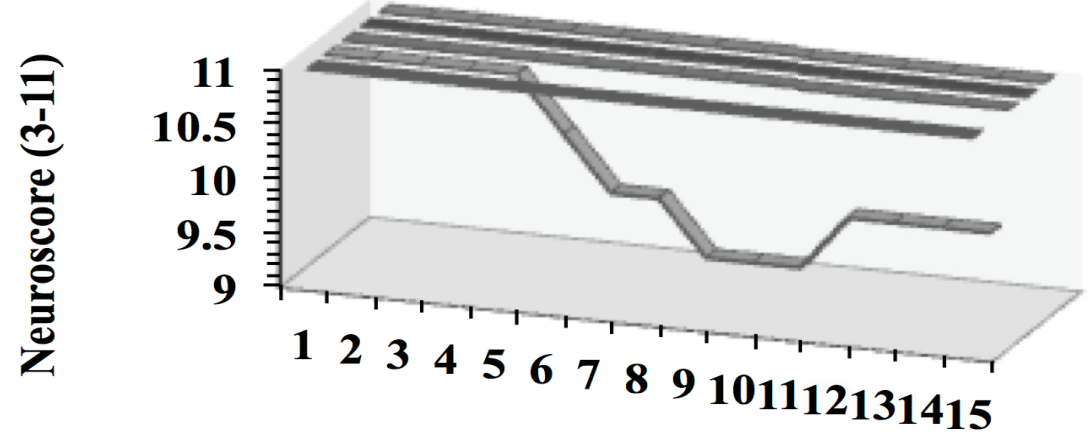

$\square$ Control

$\square \mathrm{MOI}=0.1$ (1:1)

$\square \mathrm{MOI}=\mathbf{0 . 1}$ (1:10)

MOI=10 (1:1)

$\mathrm{MOI}=10$ (1:10)

Days (post-tumor implantation) 
tumor implantation (Figure 3-22). For comparison, several animals received intratumoral injections of rNCP12.1 virus only (free virus, FV) similar to previous studies (Table 3-6). Control animals received 10 $\mu \mathrm{L}$ PBS vehicle only. Tumor sizes were calculated using ImageScope (Aperio ePathology Solutions) and were based on measurements obtained from H\&E stained tissue as well as fluorescence from GFP expressing tumors (Figure 3-23B-O). Up to 15 days post-treatment, PC treated tumor growth significantly lagged behind that of control with tumor growth reduced by as much as 69\% during the first 10 days after treatment $(p=0.013)$ (Figure 3-23A). By day 15, PC treated tumors were identical in size to control tumors while FV-treated tumors were reduced in size by $29 \%$. On day 20, all tumors had decreased in size including control tumors however in comparison to control, PC-treated tumors were $63 \%$ smaller than controls and the FV-treated tumor was $68 \%$ smaller, though not significantly. Interestingly, one animal that received pre-infected cells had no evidence of residual tumor at this time point (Figure 3-23L). Unfortunately, after day 20, tumors were unable to be analyzed as a consequence of tissue processing for this study. Whereas animals in all previous studies underwent perfusion with 4\% paraformaldehyde, animals in this study were perfused with normal saline in order to preserve tissue for qRT-PCR studies therefore brain samples were extremely fragile during cutting of tissue. Weight trends were also assessed and found to be similar to previous studies in the first week posttumor implantation (Figure 3-23P). Control animals maintained the greatest average weights with weights increasing until the end of the study whereas FV treated animals also experienced a gradual increase in weight though at a slower rate than in controls. On the other hand, weight trends in PC-treated animals gradually decreased until the end of study with average weights dropping approximately $30 \%$ from starting weights.

Neuroscores were stable early in the study with most animals maintaining a score of 11 for both control and treatment groups (Figure 3-23F). Neuroscores later in the study were lower for control and PC-treated animals, decreasing to 9 by day 25 post-treatment.

\section{Infectious virus can be recovered from brain tissue of PC-treated animals}

One advantage of using carrier cells includes the ability to not only shield virus from the immune system, but carrier cells also serve as in vivo "factories" of virus, amplifying treatment even after initial dose is given. To determine the presence of replicating virus in the CNS and in the periphery during treatment, several samples were collected at various time-points before euthanasia. Samples included sera, cerebrospinal fluid (CSF), and brain tissue. Brain tissue was homogenized in PBS and all samples were then centrifuged to remove cellular debris. Only cell-free supernatants were used in the detection of virus. One hundred microliters of each sample was added to cultured BHK cells for 1 hour at $31^{\circ} \mathrm{C}$. Samples were then removed and warm agar containing D5 was applied. At 24-36hpi, agar was removed and cells were fixed and stained to detect VSV nucleocapsid using a Rhodamine conjugated anti-VSV N monoclonal antibody. At three days post-treatment, infectious virus was recovered from homogenized brain tissue of PC treated animals (Figure 3-24A). Surprisingly, no virus was recovered after day three, however on day 24 and 25, infectious virus was again recovered from homogenized brain tissue (Figure 3-24B and C). Virus was not recovered from sera or CSF of PC-treated 


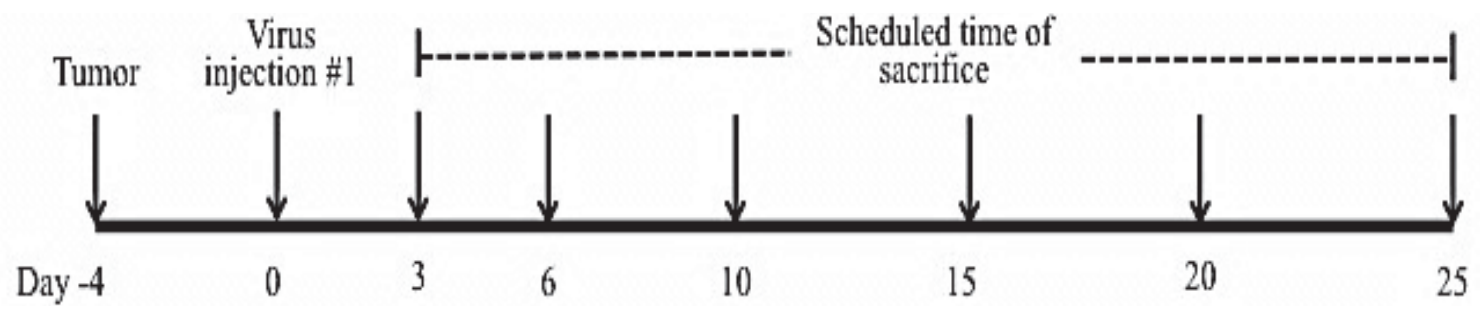

Figure 3-22. Timeline of rNCP12.1-GFP pre-infected cell study

Table 3-6. Description of treatment groups in rNCP12.1-GFP pre-infected cell study

\begin{tabular}{lc}
\hline \multicolumn{1}{c}{ Treatment Group } & Dose of rNCP12.1-GFP \\
\hline Pre-infected cells (PC) & 1000 cells infected at MOI of 3 \\
Free virus (FV) & 99 pfu free virus \\
\hline
\end{tabular}


Figure 3-23. Evaluation of rNCP12.1-GFP pre-infected F98 cells as treatment in rat intracranial model of glioma

On day four post-tumor implantation, F98 cells were infected with rNCP12.1-GFP in vitro at an MOI of 3 and 1000 infected cells were injected intratumorally. Animals were euthanized at $\mathrm{t}=3,6,10,15,20,25$ days post-treatment. (A) Comparison of tumor load demonstrated smaller tumors in PC-treated animals reaching significant levels at day 10 $(\mathrm{p}=0.01326)$. By day 15, PC-treated tumors reached the size of control tumors. FVtreated tumor was $29 \%$ smaller than controls, though not significantly. On day 20, PCtreated tumors regressed in size with tumors $63 \%$ smaller than controls while the FVtreated tumor was $68 \%$ smaller but not significantly. Representative H\&E and fluorescence micrographs of (B, D, F, H, K, M) control versus (C, E, G, I, L, N) PCtreated and $(\mathrm{J}, \mathrm{O}) \mathrm{FV}$ treated animals on $(\mathrm{B}, \mathrm{C})$ day $3,(\mathrm{D}, \mathrm{E})$ day $6,(\mathrm{~F}, \mathrm{G})$ day $10,(\mathrm{H}, \mathrm{I}, \mathrm{J})$ day $15,(\mathrm{~K}-\mathrm{O})$ day 20 . Images were taken at $0.4 \mathrm{X}$ magnification using ImageScope (Aperio ePathology Solutions). Morbidity of animals was determined by (P) Weight trends and (Q) Neuroscore.

$\S$, average tumor size of animals sacrificed at day 18 and day 20 post-treatment 


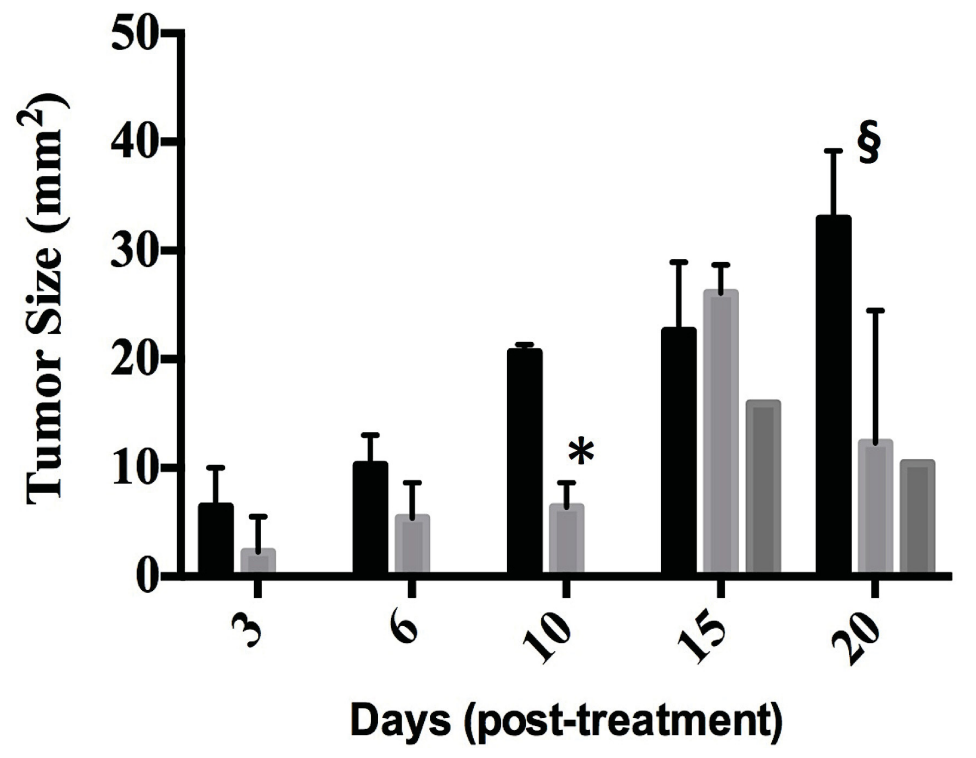

Control (vehicle) PC-treated FV-treated

$* p=0.01326$

Days (post-treatment) 


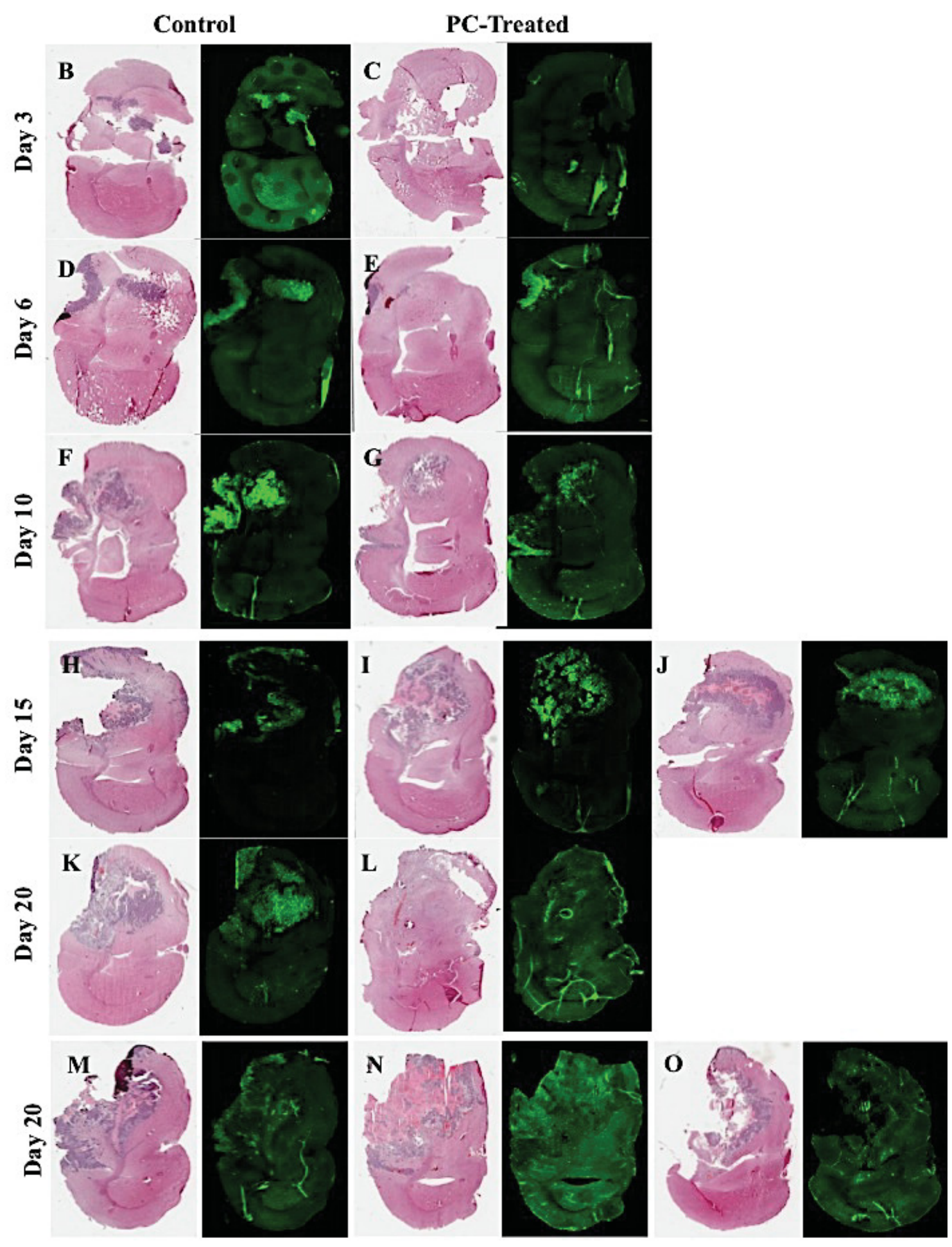

Figure 3-23. Continued 

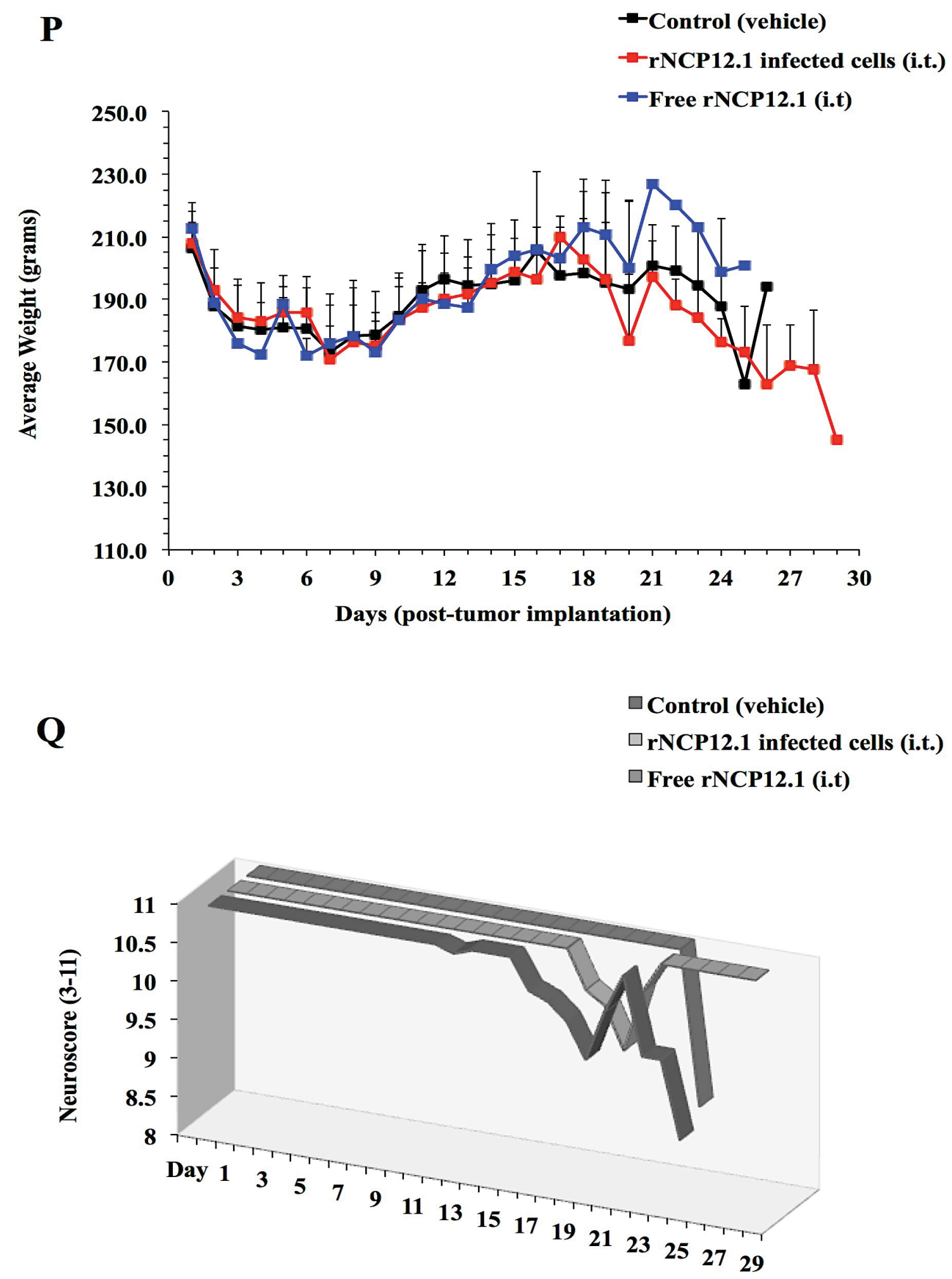

Days (post-tumor implantation)

Figure 3-23. Continued 
Figure 3-24. Detection of virus in PC- and FV-treated animals

Brain tissue and sera of treated animals were collected and used to recover infectious virus particles. Supernatant from homogenized brain tissue of animals treated with preinfected cells contained infectious virus particles at (A) day 3, (B) day 24, (C) day 25. Virus was recovered from serum of the FV-treated animal at (D) day 15. Images on the left are merges of fluorescence and bright field and images on the right are gray scale fluorescence micrographs. All images were taken at 10X magnification. Additional brain tissue was used for immunofluorescence staining for the detection of virus, tumor, and the astrocytic marker, GFAP. Representative fluorescence micrographs of (E-H) PCand (I-K) FV-treated animals show expression of GFAP (blue) localized to GFPexpressing tumor cells (green) and surrounding normal tissue. (E-G) Virus (red) in PCtreated tumors, at day three appears to be highly localized in an area surrounding the needle track within tumor tissue co-expressing GFP and GFAP. At day 15, (I-K) FVtreated tumors show virus within areas of GFP-expressing tumor. (E) Brain tissue of the untreated, non-tumor bearing hemisphere is included in order to appreciate non-specific background of green fluorescence. (H) Image of whole brain slice showing non-specific background of green fluorescence in the left hemisphere relative to GFP expressing tumor located in the right hemisphere.

E, F- 10X magnification; G, I- 20X magnification; $\mathrm{H}=0.4 \mathrm{X}$ magnification; J, K- 40X magnification 

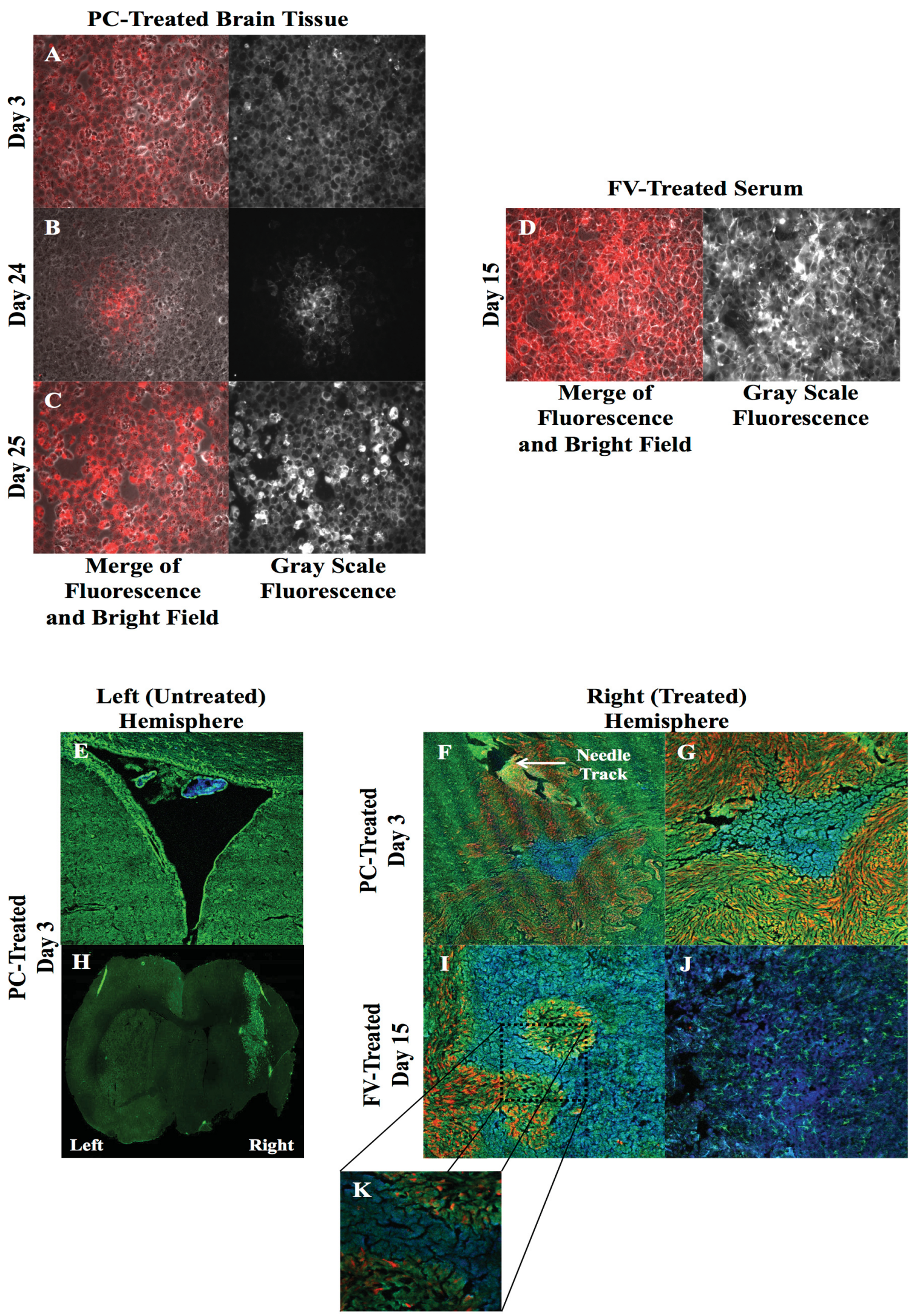
animals at any time point. Cryo-sections of brain tissue were also used for immunohistochemical analysis in the detection of GFAP and VSV-nucleocapsid. GFP expression was again used as a marker for tumor. The astrocytic marker, GFAP, was observed in multiple areas of the brain, localized both within tumor and in areas devoid of tumor. At day three post-treatment, brain tissue from PC-treated animals was positive for viral antigen. Increased VSV expression was observed in areas that correlated with high GFP expressing tumor. This area was surrounded by a GFAP positive/GFP negative (astrocytic/ non- tumor) tissue, which could correspond with normal astrocytic tissue or non-GFP expressing tumor (Figure 3-24F and G). Figure 3-24F shows that infected tumor is specifically located along the needle track, an artifact of tumor implantation. This suggests that rNCP12.1-infected cells were capable of migrating to areas of tumor to allow spread of virus within pre-established tumor. As we have seen from previous experiments, pre-infected cells lose the capacity to form tumors in vivo therefore infected cells at 3 days post-treatment are likely from previous implantation and not from treatment. Normal uninfected tissue of the left hemisphere is shown in Figure 3-24E and $\mathbf{H}$ for comparison.

At day 15, on the other hand, virus was only recovered from serum of the FVtreated animal and no virus was detected in the CSF or homogenized brain tissue (Figure 3-24D). Immunohistochemical analysis of cryosections from this same animal also demonstrated GFAP positive cells within tumor and surrounding normal tissue (Figure 3-24I-K). In addition, virus was also localized within tumor and not surrounding normal tissue.

\section{Innate and adaptive antiviral immune responses were not increased following carrier cell delivery}

Sera and CSF were also used to determine activity of both innate and acquired immunity. Using the IFN bioassay, we found that systemic IFN activity was not elevated relative to controls following treatment with pre-infected cells at any time-point. For comparison, IFN activity in serum of the FV treated animal at day 15 was elevated though not to levels observed in previous experiments (Figure 3-25A). CSF samples were also tested for presence of active IFN in the CNS, which resulted in no detectable IFN regardless of treatment (data not shown). In addition, neutralizing activity was tested using the previously described neutralization assay (Figure 3-25B and C). A control animal sacrificed on day 15 and one PC-treated animal sacrificed on day 20 were compared to levels of neutralizing antibody in the sera of FV-treated animals. Only animals receiving free virus demonstrated neutralizing activity with titers of 1000 , the same as titers confirmed in the single injection study.

In sum, the use of rNCP12.1 pretreated F98 glioma cells as a therapeutic vehicle in our rat glioma model demonstrated significant tumor load reduction. At early timepoints, it appears tumor growth was delayed relative to controls yet when tumors became more active, evident by an increase in tumor load, viral replication increased causing a second peak in tumor load reduction and viral presence at later time-points. Furthermore, 
Figure 3-25. Evaluation of innate and adaptive immune responses following treatment with pre-infected tumor cells

(A) Sera from 1-2 animals at various time points were collected and IFN activity determined by the IFN bioassay. Pre-infected F98 cells did not induce peripheral IFN activity in contrast to animals that received free virus. (B) Presence of neutralizing antibodies was first tested using Western blot analysis and sera of FV-treated animals only were found to have detectable antibodies directed against VSV G and M. (C) Neutralizing activity was evaluated using a neutralizing assay in which sera was serially diluted 2 fold and tested for the inhibition of VSV infection in BHK cells. Neutralizing titer was calculated as the inverse of the dilution found to cause inhibition of infection. + control, TN-1 (rabbit polyclonal antibody formed against intact UV-inactivated virus, nAb titer- of 800). 


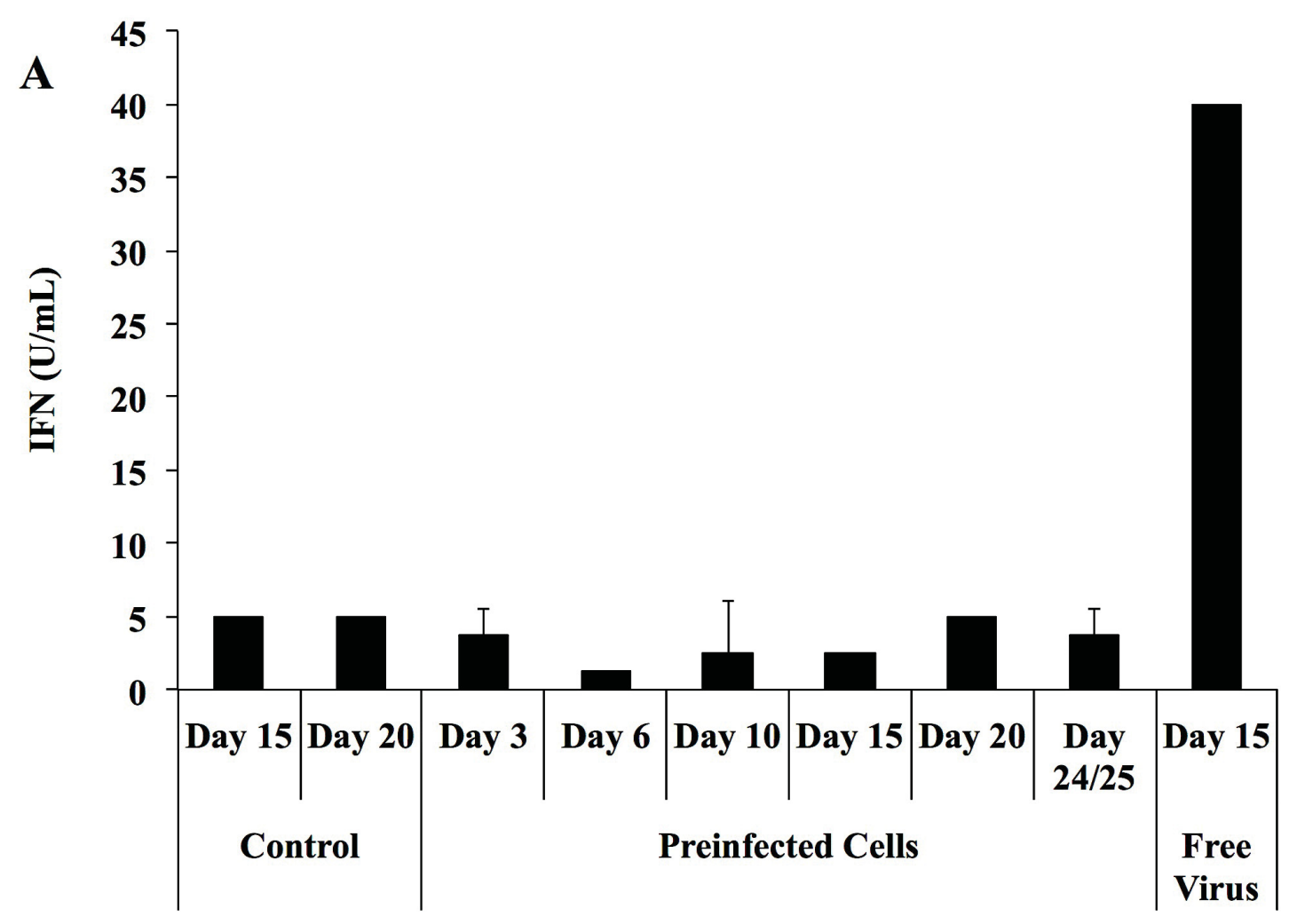

B

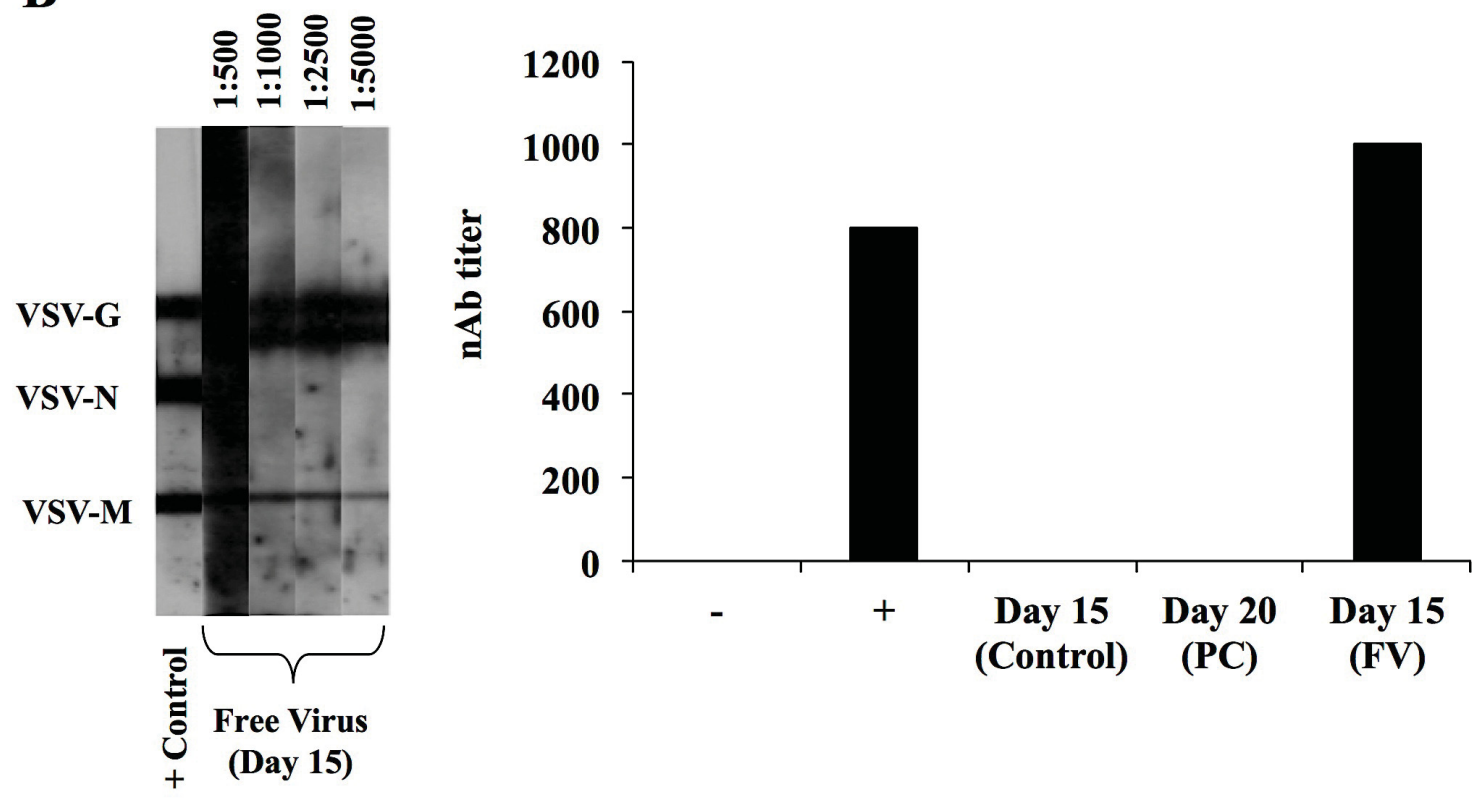


infection appeared to be localized therefore infectious virus was not present in the CSF or serum of treated animals. Not surprising, this translated to a lack of antiviral immune responses as seen by low IFN levels and absence of circulating nAbs. On the other hand, we demonstrated that direct injection of free virus elicits a robust antiviral response as seen by increased IFN levels relative to untreated animals and presence of $\mathrm{nAb}$ in the periphery. This immune response was directed against infectious virus recovered from the systemic circulation but not from brain tissue at 15 days following treatment. Despite elicited systemic immune responses, virus was still localized to areas of tumor and not normal tissue even at 15 days post infection, which corresponded with lower (though non-significant) tumor load at both 15 and 20 days post-treatment. 


\section{CHAPTER 4. DISCUSSION}

Glioblastoma multiforme is one of the most destructive of adult tumors. Prognosis is poor even when patients receive all possible therapies. Patients that are not suitable for surgical management, tend to do poorly and chemo-radiation alone may only extend life weeks to months at best resulting in a 2-year survival of only $10-18 \%[9,381]$. Median survival of patients with GBM continues to be approximately one year, even considering advancements of modern medicine. Long-term survivors (greater than 3 year survival) eventually succumb to disease as tumors typically recur and with a resistant phenotype, non-amenable to surgical or pharmacological therapy $[1,7,382]$. This shifts the focus of therapy from cure to comfort care as end of life issues become more critical. Not surprisingly, a push for therapies that have the potential to extend survival while maintaining a satisfactory quality of life have become an important focus for cancer researchers and medical practitioners of neuro-oncology, neurological surgery, and palliative care. Specifically, oncolytic viruses have moved more into the spotlight as potential therapies for cancer in general but also as a unique therapeutic agent in the treatment of high-grade gliomas. Multiple viruses along the vast spectrum of viral classes have been evaluated and have shown promise in both pre-clinical and clinical studies (reviewed in [63, 383, 384]). Ideal characteristics of oncolytic viruses being considered as therapy for GBM should include a specificity to infect tumor while maintaining the ability to and preference for replication and cytolysis in these cells over those of normal tissues. This is often challenging in the CNS especially considering the sensitive nature of neuronal tissue to damage and infection. Additionally, viruses that are naturally oncolytic often maintain the ability to infect surrounding normal tissues. Unlike non-biological therapies, viruses endure unique challenges that hinder their transition from cell culture and pre-clinical models to human patients. These challenges often involve balancing safety with optimal therapeutic efficacy, therefore, it is not surprising that understanding the immune responses elicited during and by viral therapy may help address these obstacles.

We have developed a novel viral therapy derived from a naturally selected noncytopathic variant of vesicular stomatitis virus [237,366]. VSV, an RNA virus of the order Mononegavirales is no newcomer to pre-clinical studies, and its selectivity has been tested in a number of different tumor models including breast, prostate, melanoma, liver, and brain [294, 358, 359, 361, 371, 385, 386]. VSV specificity for tumor stems from the role of the matrix protein, particularly through its ability to inhibit host gene expression especially of genes related to antiviral responses. Matrix binds at the nuclear pore complex (NPC) preventing the movement of message into the cytoplasm where it would be used to synthesize protein [275, 278]. Many tumor cells are vulnerable to these mutants due to inherent defects in their antiviral IFN responses rendering them unable to fight off viral infection while normal cells with intact IFN pathways are protected [289, 291, 292]. A number of matrix variants have been tested including those that have been genetically engineered to express defective matrix proteins to those that have developed a natural selection for growth in specific tumor cells [285, 289, 354, 355, 371].

Nevertheless, it is the inability to inhibit the innate IFN antiviral responses that is the 
main focus of tumor oncolysis. In addition to tumor specificity, other characteristics make VSV a suitable candidate for treatment of GBM. These include its quick replication cycle, ability to grow to high titers in most cell lines, broad tropism, and ease of genetic manipulation for the insertion of foreign genes or of mutations of VSV genes that help attenuate viral infection in normal cells [207, 291, 343, 344]. We have shown that our novel matrix mutant, rNCP12.1, possesses these characteristics. Specifically, viral growth and cytopathic effects represented by cell rounding vary between normal and tumor cells as well as between histologically identical tumor cells of different cell lines. Primary astrocytes have a more attenuated growth pattern with rNCP12.1 as well as wtVSV in comparison to growth in rat glioma cells however this mutant produced 10 fold less virus relative to wildtype. Cytotoxicity was also attenuated and primary astrocytes infected with rNCP12.1 had minimal cell rounding. On the other hand, in the majority of glioma cell lines, differences, though minimal, in growth of rNCP12.1 relative to wildtype virus were most noticeable in C6 earlier during infection with titers lagging behind but eventually catching up to wildtype titers at later timepoints. Human glioma cell lines also varied in their sensitivity to rNCP12.1 infection. For instance U87, known to have chromosomal aberrations in the IFN-I gene, is extremely sensitive to rNCP12.1 infection, with cells forming large syncytia indicative of membrane fusion that is characteristic of VSV infection in vitro. T98G human glioma cells, on the other hand, have an intact IFN signaling which correlated with an overall resistance to rNCP12.1 infection evident by minimal expression of viral antigen and almost negligible cell rounding.

With these results in mind, we were, naturally curious about the IFN activity in cells of our rat glioma model in order to determine if in fact IFN activity or lack thereof corresponded with cytopathic attenuation. Using semi-quantitative RT-PCR to check for the presence of IFN $\beta$ mRNA, we observed an induction of IFN $\beta$ following wtVSV infection in rat fibroblasts, though at extremely low levels whereas rNCP12.1 elicited a robust signal for IFN $\beta$ message at both 6 and 12hpi. IFN $\beta$ signal following wtVSV infection in $\mathrm{C} 6$ rat glioma was negligible while rNCP12.1 increased IFN expression to levels comparable to that found in rNCP12.1 infected rat fibroblasts; however, expression was not sustained and substantially dissipated by $12 \mathrm{hpi}$. Quantitative analysis provided even stronger evidence of differences between normal and tumor cells to elicit IFN responses. Though wtVSV induced some level of expression in all cell types, rNCP12.1 caused a much greater level of induction. This induction extended into tumor cell lines, as well, though at exponentially lower levels than normal cells. These results in most studies would be interpreted as corresponding to equivalent levels of protein synthesis. In order to determine whether expression levels indeed translated into active protein, we tested for the presence of biologically active IFN levels as seen by antiviral activity in the supernatant of infected cells and we found that wtVSV caused production of very low amounts of active IFN only in primary astrocytes. Robust antiviral IFN activity however was observed in normal rat fibroblasts infected with rNCP12.1. These levels gradually increased over time showing that normal astrocytes like rat fibroblasts are able to amplify signal with time. Not surprisingly, IFN activity was also observed in tumor cells however they lacked the ability to consistently amplify signal (C6, F98) or to elicit a robust production of IFN early during infection (F98). 
Generally, mRNA accounts for less than half of protein abundance and it is suggested that the ratio of mRNA abundance to that of protein leans more toward a higher prevalence of protein. This ratio is sensitive to cellular conditions and often depends on the specific role a protein plays in cellular activity [370]. Nonetheless, it is assumed that if a specific gene transcript is detected, likely the corresponding protein will be detected also. An interesting observation from these data was the imbalance between gene expression and protein activity in each cell line with mRNA abundance being more prominent than protein activity. More specifically, there appeared to be much tighter regulation on the production of active IFN by the primary astrocytes. There are a number of potential levels of regulation that could account for this divergence. As we know, VSV matrix protein inhibits nucleocytoplasmic transport (NCT) of message [278]. This is evident in our results with wtVSV, however mutants should be defective in their binding at the NPC therefore allowing transcripts to be exported from the nucleus. This in fact appears to be true, however, there may be a difference in sensitivity to this inhibition that is cell specific. For instance, a lower level, relative to other cells tested, of matrix binding to NPCs may be all that is required in astrocytes to block NCT. Regulation of post-transcriptional events including increased stability of IFN transcripts might also be at play. Several studies have indeed addressed mechanisms that affect the stability of IFN transcripts following infection. Johnsen et al demonstrated that IFN $\beta$ transcripts possess a 3' untranslated region that contains sequences involved in controlling mRNA stability. During infection or in the presence of synthetic inducers of the antiviral pathway such as $\mathrm{pI}$ C, IFN $\beta$ transcripts become more stable, enhancing production of protein during infection, which increases the likelihood of a protective antiviral state [387]. It is possible that at this time point we are observing the effects of more stable mRNA in combination with continued cellular control over translation. Interference at the translational and posttranslational level is also a possibility. These could include alterations in the half-life of protein due to increased degradation or protein stability as well as post-translational modifications rendering the protein inactive.

From these results, the question becomes, whether or not more is better; is overabundance of active IFN necessarily biologically relevant? Considering astrocytes are a vital component of the cellular network in the central nervous system, it is not surprising that there would be a tighter control over the availability of biologically active IFN. In fact, outside of its role in antiviral responses, IFNs are also important in cell survival serving as a major inducer of apoptotic responses [23, 330]. In comparison to neuronal cells, astrocytes do maintain some level of competency to proliferate and are the most important CNS cell that responds to brain trauma by proliferating in a process known as gliosis [388, 389]. Despite this proliferative capacity, uncontrolled promotion of cell death would be detrimental therefore controlling IFN could serve as a survival mechanism in the face of infection. A better understanding of the mechanisms involved in controlling IFN activity in primary astrocytes could aid in addressing these points. Future studies might first test whether control of active protein produced is the same as control of total protein in astrocytes. This can be done by ELISA analysis to quantify total protein concentration of IFN. If total protein adheres to the same pattern, it might be worthwhile to determine whether there is a noticeable gradient of mRNA from the nucleus to the cytoplasm as this would point more toward NCT as a cause for the 
disproportion between gene expression and protein activity.

IFN defects can happen at multiple points in the antiviral response pathway from the production of IFN to IFN sensitivity [331-333]. Therefore, it is possible that even though certain cells may not produce active IFN, they may in fact retain the ability to respond to IFN. This response is evident by the promotion of downstream interferon stimulated genes (ISGs) that amplify the IFN pathway, promoting the protection of cells during viral infection. We next tested the sensitivity of cells to exogenous IFN as determined by their ability to inhibit virus propagation in cell culture and found that primary astrocytes are highly sensitive to antiviral effects of IFN with protection observed at IFN levels $<5 \mathrm{U} / \mathrm{mL}$. On the other hand, glioma cells showed variable levels of sensitivity with F98 being completely resistant and C6 glioma having a more intermediate level of sensitivity requiring higher levels of exogenous IFN to create protection (summarized in Table 4-1). Generally speaking, cells that respond to IFN but do not produce IFN may likely have defects that are concentrated upstream of IFNAR activity. On the other hand, cells that produce IFN but are not able to respond to IFN may harbor more defects downstream of IFN binding to its respective receptor, such as in JAK/STAT signaling.

The focus has mostly been on whether individual cell types alone could elicit protective IFN responses by an endogenous pool of induced IFN. Endogenous levels become more important in cell culture however, in a living system, we must take both pools of IFN into account, IFN produced by that specific cell population and IFN produced by surrounding cells of different types. Hence, the combination of these data, which includes IFN production by cells correlated with viral titers and cell specific viral induced CPE versus protection by exogenous IFN suggest that they are both important.

In adding to the above studies, we also performed more in depth analysis of IFN

Table 4-1. Summary of in vitro results of VSV in normal and tumor glial cells

\begin{tabular}{|c|c|c|c|c|c|c|}
\hline \multirow{2}{*}{$\begin{array}{c}\text { Measurable } \\
\text { Response to } \\
\text { Infection } \\
\end{array}$} & \multicolumn{2}{|r|}{ PRA } & \multicolumn{2}{|c|}{ C6 } & \multicolumn{2}{|c|}{ F98 } \\
\hline & wt vs. & rNCP12.1 & wt vs. & P12.1 & wt vs. & P12.1 \\
\hline Cell Rounding & ++ & - & ++++ & +++ & ++++ & ++++ \\
\hline Cell Viability & + & ++++ & ++ & ++ & + & + \\
\hline Viral Yield & ++ & + & ++++ & $\begin{array}{l}+++/ \\
++++\end{array}$ & ++++ & ++++ \\
\hline $\begin{array}{l}\text { IFN } \\
\text { transcription* }\end{array}$ & ++ & ++++ & ++ & +++ & + & ++ \\
\hline IFN bioactivity* & $+/-$ & +++ & - & +++ & - & ++ \\
\hline
\end{tabular}

Note: Scale of + to ++++ signifies lowest to highest for each measure of response to infection; -, negligible

* IFN transcription and bioactivity are relative to control cells (FR, rat fibroblasts) 
responses as determined by induction of specific ISGs, several of which have been studied more than others as responders of VSV infection. Van den pol and associates tested the stimulation of genes in several human glioma cell lines (U87, U118, U373, A172, M059) as well as in primary glioma tissue compared to normal brain tissue and found that their glioma-adapted VSV mutant, VSV-rp30a (MOI of 2) and treatment with exogenous IFN $(100 \mathrm{U} / \mathrm{mL})$ both demonstrated significant increases in expression levels of IFN $\beta$ and MxA $6 \mathrm{~h}$ post treatment [371]. Another key study by Barber and associates involving some of the first engineered attenuated matrix variants of VSV (AV1 and AV2) showed that wtVSV blocks transcription at the level of secondary ISG responders such as GBP1 and MxA while mutants allow, and actually, induce expression levels of the same transcripts [289]. Expression of these ISGs goes on to activate tertiary responders (e.g IFN $\alpha 4$ ), which augment antiviral activity. This study tested ISG responses in an ovarian carcinoma cell until $12 \mathrm{hpi}$. Though our studies veered from the above studies in tumor cell type (human glial versus ovarian carcinoma versus rat glial), time post infection (6hpi versus $12 \mathrm{hpi} \mathrm{versus} \mathrm{24hpi)} \mathrm{and} \mathrm{specific} \mathrm{mutant} \mathrm{virus} \mathrm{(vsv-rp30} \mathrm{versus} \mathrm{AV1} \mathrm{and}$ AV2 versus rNCP12.1), overall, we observed increases in expression levels by our mutant in comparison to wildtype. Several differences in our results included that we did not see a total inhibition of secondary interferon responders however our study was carried out 12 hours after those showing inhibition and therefore it is possible that these responders eventually did become expressed as cells began to overcome the block of wtVSV. Several individual ISGs were also noted to not follow the expected increases after mutant infection in our study. Altogether, our non-tumor cell line, primary rat astrocytes, followed a pattern of ISG induction almost identical to that of control rat fibroblasts whereas glioma cell lines varied from this pattern and even from each other. Whether these differences affect rNCP12.1 related oncolysis of glioma cells will require additional studies. At any rate, though specific interpretation of the intricacies of these patterns is beyond the scope of the project, several ISGs are worth mentioning in detail.

RIG-I, for instance, is an important component of antiviral signaling through its role as a pattern recognition receptor. Particularly, RIG-I has been shown to be indispensible in the activation of antiviral IFN activity in fibroblasts after VSV infection, with RIG-I knockout cells having up to 100 fold higher viral titers than normal cells [306]. RIG-I is constitutively active and has been shown to be most elevated in astrocytes at 4hpi returning to basal levels by 8 hpi [372]. Our analysis of expression levels in astrocytes supported this as both PRAs and FR cells had very low levels at $24 \mathrm{~h}$ following wtVSV infection. In contrast, both glioma cell lines demonstrated a notable elevation following wild-type infection at the same time point. Even more, expression in all cell types following rNCP12.1 was significantly elevated at 24hpi. Though it is unknown whether this expression at $24 \mathrm{hpi}$ is due to a delayed induction of RIG-I or rather a constant, prolonged induction, it is of note that this contradicts what is known about RIG-I activity this late in infection. Many cells display autonomous control of RIG-I activity, specifically by a splice variant, which is upregulated during viral infection or following exposure to Type I IFN [390]. This splice variant is determined at the transcriptional level and amplification of RNA in cells treated with IFN or after viral infection shows both wildtype and variant bands. RIG-I responses have been shown to be inhibited by the heterodimer formation of the RIG-I splice variant with the wildtype 
form as a negative feedback mechanism to protect cells from unbalanced production of IFN [390, 391]. Our results specifically showed that wtVSV and rNCP12.1 induction of RIG-I was highest in C6 glioma but not normal cells. Therefore, future studies might address whether this variant is present in our glioma cell lines specifically in C6 cells thus possibly causing the observed levels of IFN gene expression and ultimately IFN activity. In addition, evaluation of RIG-I expression levels at earlier time points might reveal whether rNCP12.1 prolongs RIG-I expression or whether expression is actually delayed.

Expression of plscr1 was also notable following infection. This ISG is known to affect survival and terminal differentiation of myeloid cell lines [326]. The ability of plscr-1 to attach and insert into the cell plasma membrane is interesting as plscrl contains the PPXY N-terminus motif just as is found in the N-terminus of VSV matrix, which has been shown to interact with SH3 and WW domains found in a host of cellular proteins. Moreover, depending on post-translation palmitoylation, plscr-1 migrates to the nucleus where it binds to DNA as a regulator of transcription, therefore IFN-induced gene expression. It may be possible that, during VSV infection, especially events during the replication cycle such as assembly and budding where matrix is associated with the cell membrane, competition for similar cellular proteins might occur between matrix and plscr1 during viral infection. Except for F98 levels, our studies revealed very low expression in all other cell types regardless of infecting virus. F98 glioma demonstrated 10 fold higher expression levels of plscr1 following rNCP12.1 infection. Therefore, aside from results in F98 cells, expression levels in all other cell lines are in contrast to those observed by Barber et al in which plscr1 expression was inhibited by wtVSV but induced starting at 6hpi with attenuated VSV strains [289]. It is possible that like RIG-I, some cells especially non-tumor cells regulate the overexpression of plscrl whereas F98 cells are unable to, especially considering their role in effecting sensitivity to viral infection. Additionally, it is possible that plscr1 levels were indeed elevated at earlier time points and, like RIG-I, should decrease back to lower basal levels at later time points. Therefore, evaluation of plscr 1 expression at earlier time points leading up to 24hpi would allow us to determine which is more likely. It would also be of interest to see if in fact there are differences in localization of plscrl as a result of VSV infection particularly of displacement of plscr1 from the cell membrane by competition with matrix for binding of cellular proteins and whether this relationship correlates with IFN activated responses.

Lastly, IRF-1, an inducer and effector of the IFN pathway, is known to be directly upregulated in response to VSV infection, even in the absence of IFN [392]. IRF-1 levels are observed during states of cell cycle arrest as well as during apoptosis. IRF-1 increases expression not only of Type I IFN but also of a subset of ISGs including Mx1 and 2, GBP1, OAS, PKR, and RIG-I [310]. Sgorbissa et al demonstrated that U87 and T98G, two human glioma cell lines that lie on the opposite ends of the IFN spectrum, expressed biphasic elevations of IRF1 following treatment with IFN $\alpha$ at $6 \mathrm{~h}$ and later at 24h [331]. IRF1 expression went on to induce expression of TRAIL, a known activator of apoptosis, in T98G cells but not in U87. In our studies, glioma cells expressed similar levels of IFN $\alpha 4$ regardless of infecting virus. IRF-1 expression also demonstrated this 
same non-specific pattern in F98 as well with wtVSV inducing almost identical levels of expression as rNCP12.1. This demonstrated that the expected elevations in IRF1 expression following mutant infection was not observed in F98 glioma and that the relationship between IFN $\alpha 4$ and IRF1 induction was still intact. On the other hand, IRF1 levels showed a switch in expression with wtVSV inducing a higher expression over rNCP12.1 in C6 glioma even more so than increases in expression following rNCP12.1 in normal cells. This may be that IFN independent induction of IRF1 following wtVSV infection in C6 cells is more important following wtVSV than in other cell types.

Obviously, a thorough explanation for these results would require additional studies in order to determine the meaning of these patterns of induction and how they relate to the overall IFN phenotype of each cell line. In combination with IFN activity and protection assays, unique cell specific IFN phenotypes are revealed and could possibly serve as predictors of cells that may likely respond to VSV oncolytic therapy.

As we moved from in vitro studies into an immunocompetent animal model of glioma, we saw positive tumor response to rNCP12.1 therapy even after only a single injection of virus. This tumor reduction, though highly significant was not sustainable and tumors eventually recurred at an expedited rate over untreated animals so much that at the time of euthanasia, tumors were virtually the same size as animals that never received virus. Similar results have been observed in other studies particularly testing naturally oncolytic viruses like VSV. In their evaluation of Semliki Forest Virus as treatment for glioma, Maatta et al demonstrated that when using an immunocompetent model for systemically administered OV, tumor growth is inhibited early during treatment resulting in significantly smaller tumors than controls; however, treated tumors do eventually catch up to the size of untreated tumors at an accelerated pace. On the other hand, immunocompromised animals lacking an intact antibody response did not demonstrate this type of recurrence [393]. Non-published data from the same group, observed that in a different immunocompetent syngeneic model of glioma, SFV was more effective at the same dose of virus suggesting that specific tumor model characteristics play an important role in therapeutic efficacy [394]. Nonetheless, our evaluation of host innate and adaptive immune responses following intratumoral rNCP12.1 confirmed an obvious increase in both IFN and in VSV neutralizing activity. Specifically, neutralizing activity was observed at time points that coincide with natural progression of immune responses against VSV [395].

The cause for accelerated tumor recurrence in the presence of heightened immune responses is likely multi-factorial. As we stated, injury to normal brain tissue can activate proliferation of normal astrocytes. It is possible that whatever factors trigger this response can be a cause of increased growth of astrocytic tumors as well. Changes in the tumor microenvironment caused by virus may additionally serve as a factor and several studies have revealed key changes that occur with viral therapies. For instance, Kurozumi et al showed that oncolytic viral infection promoted enhanced leakage of vessels when administered intratumorally even over the pro-angiogenic responses already encouraged by the tumor itself. Enhanced tumor perfusion opens the flood gates for the movement of antiviral immune responders capable of inactivating virus therefore 
decreasing viral effect [396]. Another possible cause of enhanced growth could be due to tumor-acquired resistance to virus. With chemotherapeutic agents, resistance to therapy is a common occurrence during treatment of highly malignant brain tumors. It is possible that these cells are also capable of acquiring resistance to viral therapy in the same manner. In addition, cells have been known to acquire a tolerance to virus. As a matter of fact, many of the attenuated strains of VSV were discovered during persistent infections [356, 357]. Previous studies in our lab have demonstrated the ability of VSV to establish persistent infections in cell culture. This was even demonstrated in several studies involving the F98 glioma cell line (data not shown). Persistence of infectious virus in our tumor model would likely terminate oncolysis, therefore, increasing the possibility of tumor proliferation.

To test whether controlling immune mediated neutralization of virus could enhance tumor reduction and decrease or possibly inhibit later accelerated tumor growth, we performed another evaluation of intratumoral rNCP12.1 but by administering two separate injections. Two treatment arms were designed, one group receiving 2 injections of the same serotype (Ind/Ind) and the other receiving 2 injections, one from Indiana serotype and the other from New Jersey serotype (Ind/ NJ) of VSV. It is generally accepted that neutralizing activity against one serotype does not cross react with the other. More accurately, cross reactivity of one serotype over another was found rather to be dependent upon several factors: route of administration; amount of viral antigen present; co-administration with immune adjuvants. Live virus given into the peritoneal cavity only activates non-cross reactive antibodies whereas intravenous virus or virus given at high levels especially over a prolonged period of time (e.g. persistent infection) activates antibody with an assymetrical cross reactivity. This means that antibodies produced by the New Jersey serotype are more cross reactive to Indiana than are Indiana antibodies against the New Jersey serotype [397]. To our knowledge, no studies have shown whether an assymetric cross reactivity exists when virus is administered in the CNS. Based on our results, 2 doses of rNCP12.1 were just as effective at reducing tumor as one injection at earlier time points (up to 15 days). Differences at later time points showed that animals treated with the same serotype experienced a robust recurrence of tumor while tumors of animals receiving a switch in serotype for the second dose maintained smaller tumors until the time of euthanasia. Survival of both animals was identical. It was not determined in this experiment whether neutralizing antibodies were produced in response to virus or whether these responses possessed cross reactivity between serotypes. In addition, each injection was given 5 days apart, day 5 post-tumor implantation for the first injection and day 10 for the second injection. Therefore, presence of a substantial neutralizing antibody population likely had not occurred. Future studies should aim to determine the time course of $\mathrm{nAb}$ production from virus administration to neutralizing activity in order to optimize anti-tumor effect from the second dose of therapy.

As mentioned before, the immune system is a double-edged sword in which immune responses activated by virus can also serve as key anti-tumor effectors that contribute to what is known as the bystander effect seen during therapy. This means that non-infected tumor cells are killed by enhanced anti-tumor immunity triggered by an 
upregulation of immune mediators that, before viral infection, were not strong enough in their response to eliminate tumor. The other side of the sword involves the early elimination of viral treatment in an effort to protect the infected host. By amplifying the signal of danger to surrounding immune cells, viruses are able to disrupt the state of immune tolerance created by tumors and even increase visibility of tumor associated antigens (TAAs) that otherwise would go unnoticed [398].

Several studies have suggested the use of carrier cells as a mobile manufacturer of virus, shielding its product from immune regulators during transit. As tumor cells should retain susceptibility to infection, viral dose would theoretically be amplified during transit, as they home to sites of tumor attracted by their preferred tumor niche [197, 399]. One such study tested a combination of immunosuppressants with cell carriers of an oncolytic poxvirus in the treatment of a peritoneal carcinomatosis mouse model. MC38, autologous murine colon carcinoma cells were used as carriers. This therapy not only enhanced survival but also promoted recovery of virus from tumor tissue confirming the continued replication of virus within tumor even at eight days post treatment [400]. We tested the use of autologous F98 glioma cells as vehicles for rNCP12.1 in the treatment of glioma in our immunocompetent Fischer rat model. Tumors responded to treatment significantly as demonstrated by a reduction in tumor load until day 15 at which point treated tumors were equal in size to those of control animals. Though comparisons of tumor size following this time point were unable to be determined due to quality of tissue samples, of note, at day 20, one animal showed complete regression of tumor while tissue of other animals demonstrated areas of high cellular infiltrate on H\&E that did not correlate with GFP expression on fluorescence microscopy. In addition, analyses of immune responses proved that carrier cells were effective in their ability to shield virus from potential antiviral responses. There was an absence of neutralizing antibody in the serum of PC-treated animals even at 20 days post-treatment, despite recovery of virus from brain tissue at the same time point. This was opposite of what was observed in FVtreated animals which were positive for IFN and neutralizing antibodies in the periphery. Virus in these animals was not recovered from brain tissue but rather from systemic circulation. It appears from these results as well as of previous experiments that neutralizing antibody directly correlates with the size of tumor and that the concept of "the more, the better" pertaining to IFN activity during viral treatment may not necessarily be a marker for predicting effective response to therapy (See Table 4-2 for summary of in vivo experiments).

Another theory that has been proposed concerning autologous tumor carrier cells is that they could possibly initiate or encourage anti-tumor activity. A number of very successful therapies are based on this possibility including monoclonal antibodies that are directed against specific tumor proteins or those of the tumor microenvironment (i.e. VEGF, Her2). As a preliminary study, we evaluated immune recognition of tumor cells by testing for the presence of anti-tumor antibodies. Using Western blot analysis, we analyzed serum for the presence of systemic antibodies that may have been produced against F98 specific tumor antigens during treatment (See Appendix A). Results revealed a pattern of tumor antigen recognition that was specific to FV-versus PC-treated animals. The majority of antigens more immunogenic in FV-treated animals were antigens that 
Table 4-2. Summary of in vivo experiments of rNCP12.1 in the syngeneic F98 glioma/ Fischer rat model

\begin{tabular}{|c|c|c|c|c|}
\hline $\begin{array}{c}\text { Study } \\
\text { Treatment }\end{array}$ & $\begin{array}{l}\text { Acute, } \mathbf{A} \\
\text { or } \\
\text { Survival, } \\
\text { S }\end{array}$ & $\begin{array}{c}\text { Tumor Load } \downarrow ? \\
(\%)\end{array}$ & $\begin{array}{l}\text { Significant } \\
\text { Survival ? }\end{array}$ & $\begin{array}{c}\text { Median } \\
\text { Survival }\end{array}$ \\
\hline Single injection: & $\mathrm{A}$ & Yes $(65 \%)$ & N/A & $\mathrm{N} / \mathrm{A}$ \\
\hline $\begin{array}{l}10 \text { pfu rNCP12.1 - } \\
\text { GFP }\end{array}$ & $\mathrm{S}$ & No & Yes & $\begin{array}{c}23 \text { (C) vs. } \\
29 \text { (Tx) }\end{array}$ \\
\hline $\begin{array}{l}\text { Single injection w/ } \\
\text { Alzet Pump: } \\
\text { High Dose, HD; } \\
\text { Medium Dose, MD; } \\
\text { Low Dose, LD }\end{array}$ & S & No & $\begin{array}{c}\text { Yes } \\
\text { (CV vs LD) }\end{array}$ & $\begin{array}{l}21(\mathrm{CV}) \text { vs. } \\
27(\mathrm{LD})\end{array}$ \\
\hline $\begin{array}{l}\text { Double Injection/ } \\
\text { Serotype Switch: } \\
10 \text { pfu rNCP12.1 (I/I) }\end{array}$ & A & $\begin{array}{c}\text { Yes } \\
(63 \%, \mathrm{I} / \mathrm{I} \\
65 \%, \mathrm{I} / \mathrm{NJ})\end{array}$ & N/A & N/A \\
\hline $\begin{array}{l}\text { and } 109 \text { pfu rNCP12.1 } \\
(\mathrm{I} / \mathrm{NJ})\end{array}$ & $\mathrm{S}$ & $\begin{array}{c}\text { No } \\
(54 \%, \mathrm{I} / \mathrm{NJ})\end{array}$ & Yes & $\begin{array}{l}21(\mathrm{C}) \text { vs. } 24 \\
(\mathrm{I} / \mathrm{I} \text { and } \mathrm{I} / \mathrm{NJ})\end{array}$ \\
\hline $\begin{array}{l}\text { Pre-infected Tumor } \\
\text { Cell Carriers: } \\
10^{3} \text { cells infected at } \\
\text { MOI of } 3\end{array}$ & $\mathrm{~N} / \mathrm{A}^{*}$ & Yes** $^{* *}(69 \%)$ & N/A & $\mathrm{N} / \mathrm{A}$ \\
\hline
\end{tabular}

*This experiment was a time course in which animals were sacrificed at 3-5 day intervals.

**Tumor load was significantly decreased up to 10 days following treatment; Later time points will need to be repeated

N/A, not applicable; Tx, treated; C, control; CV, control with vehicle; LD, low dose; I/I, Indiana/ Indiana; I/NJ; Indiana/ New Jersey 
were not recognized in the other treatment groups whereas most antigens that were more immunogenic in PC-treated animals were also immunogenic in the other groups though at much higher levels of reactivity. Of note, the greatest level of tumor antigen recognition was observed at day 15 following treatment for both FV- and PC-treated animals. These preliminary data are encouraging and more in depth analysis into the importance of these immunogenic antigens could provide a platform for more sophisticated immunotherapeutic adjuvants for the treatment of high-grade brain tumors.

In summary, we have revealed unique cell specific patterns of gene expression in various cell types that must be taken into account in order to determine if rNCP12.1 would be an optimal therapy in that system. Just as every high-grade glioma does not respond as anticipated to temozolamide (TMZ), the standard chemotherapeutic agent for GBM, there is a subset of tumors especially sensitive to TMZ based on mutations in the DNA repair enzyme, MGMT. Therefore a pre-determined molecular signature can be extremely helpful in making sure that a particular treatment will have optimal effect as an anti-tumor agent. Similarly, determination of proposed IFN phenotypes that are defined by the ability of cells to produce IFN and respond to IFN, evident by the ability to upregulate the expression of particular ISGs as well as to create an antiviral state following exposure to exogenous IFN, could prove to be just as important in determining whether a specific tumor is right for rNCP12.1 therapy (Figure 4-1). The push for a less histologically oriented and more molecular signature based classification system of gliomas stems from the importance of these molecular signatures on therapeutic outcomes. Supporting evidence for this concept as it relates to VSV oncolytic therapy is evident in several studies that confirmed differences in response to viral therapy based on IFN expression profiles. One such study involved the evaluation of another RNA virus, Semliki Forest virus (SFV) as a cancer therapeutic in several cancer models including melanoma and glioma. GL261 and CT-2A glioma cells were shown to be producers of and responders to IFN and therefore were protected against in vitro infection with SFV. When pre-infected GL261 were implanted intracranially, these cells retained the ability to form aggressive intracranial tumors in immunocompetent mice [401]. On the other hand, a study by Ruotsalainen et al demonstrated the IFN resistant cell line CT26 were highly susceptible to SFV infection in a syngeneic model of colon carcinoma. This is not to say that VSV based OVs would not work in the face of IFN sensitive cells [401]. As Alain et al have shown, tumor models responsive to IFN benefit from the addition of adjuvant immunomodulatory therapies such as rapamycin which inhibits IFN activity. They demonstrate in this study that though rapamycin decreases the production of IFN, levels of IFN produced are still able to protect normal tissue resulting in a significant increase in survival over virus alone [193].

As oncolytic viruses continue down the road to clinical studies, it is important to remember that in vitro results as compelling as they may be are not always relevant in living models. As is the case of rNCP12.1 viral therapy, neutralizing activity proved to be as important if not more to IFN phenotype in the potential to reach optimal efficacy in vivo. In addition the ability to enhance anti-tumor activity may prove to be the most important factor related to long-term outcome measures resulting from viral therapy. 


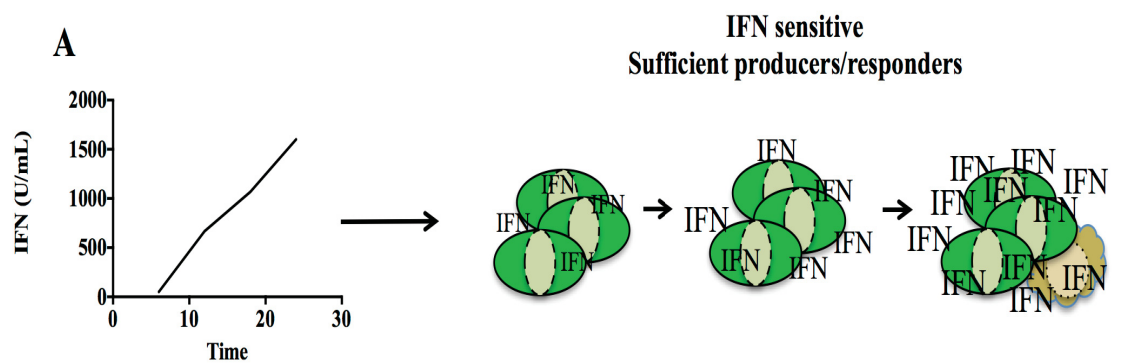

Examples
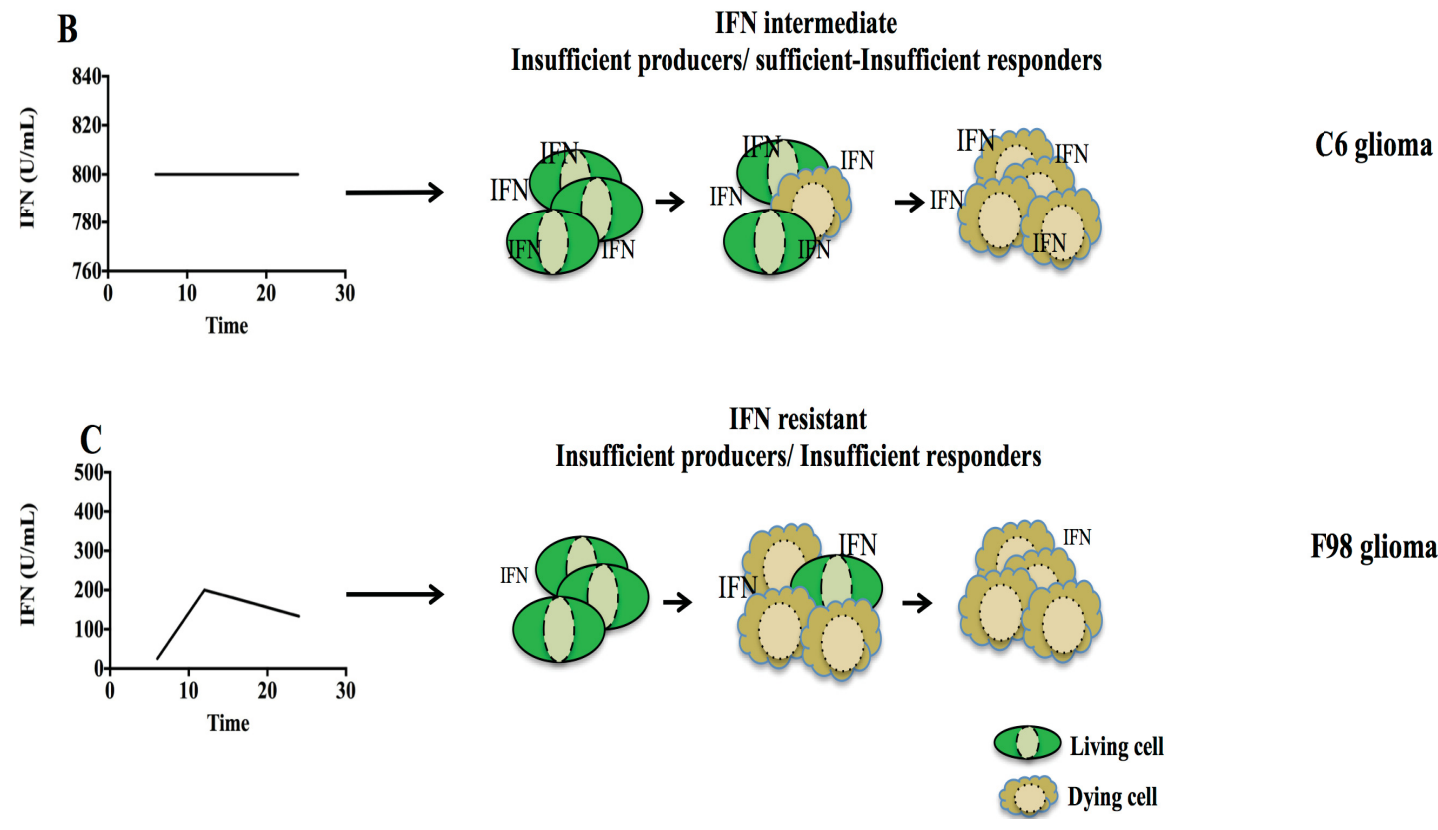

F98 glioma

Figure 4-1. Interferon phenotypes of glial cells in response to rNCP12.1 infection In summary, these normal and tumor rat glial cells have unique IFN phenotypes. These phenotypes can be described as either (A) IFN sensitive in which cells sufficiently produce and respond to exogenous IFN, (B) IFN intermediate in which cells insufficiently produce IFN at levels unable to create an antiviral state and they respond to exogenous IFN at much higher levels than are needed to protect normal cells. It is unknown whether this protective state is sustainable past 24 hours, (C) IFN resistant cells that insufficiently produce and respond to exogenous IFN. 


\section{LIST OF REFERENCES}

1. Krex, D., et al., Long-term survival with glioblastoma multiforme. Brain, 2007. 130(Pt 10): p. 2596-606.

2. Brat DJ, e.a., Pseudopalisades in glioblastoma are hypoxic, express extracellular matrix proteases, and are formed by an actively migrating cell population. Cancer Res 2004. 64: p. 8.

3. Louis DN, e.a., The 2007 WHO Classification of Tumours of the Central Nervous System. Acta Neuropathol 2007. 114: p. 13.

4. Dolecek TA, P.J., Stroup NE, and Kruchko C, CBTRUS Statistical Report: Primary Brain and Central Nervous System Tumors Diagnosed in the United States in 2005-2009. Neuro-Oncology 2012. 14: p. 50.

5. Ohgaki H, K.P., Epidemiology and etiology of gliomas. Acta Neuropathol, 2005. 109: p. 16.

6. Goodenberger ML, J.R., Genetics of adult glioma. Cancer Genet 2012. 205: p. 9.

7. Wen PY, K.S., Malignant gliomas in adults. N Engl J Med 2008. 359: p. 16.

8. Sanai, N., et al., An extent of resection threshold for newly diagnosed glioblastomas. J Neurosurg, 2011. 115(1): p. 3-8.

9. Bloch $\mathrm{O}$, e.a., Impact of extent of resection for recurrent glioblastoma on overall survival. J Neurosurg 2012. 117: p. 7.

10. Walker, M.D., et al., Evaluation of BCNU and/or radiotherapy in the treatment of anaplastic gliomas. A cooperative clinical trial. J Neurosurg, 1978. 49(3): p. 33343.

11. Johnson, D.R. and S.M. Chang, Recent medical management of glioblastoma. Adv Exp Med Biol, 2012. 746: p. 26-40.

12. Stupp, R., et al., Radiotherapy plus concomitant and adjuvant temozolomide for glioblastoma. N Engl J Med, 2005. 352(10): p. 987-96.

13. Louis, D.N., E.C. Holland, and J.G. Cairncross, Glioma classification: a molecular reappraisal. Am J Pathol, 2001. 159(3): p. 779-86.

14. Shirahata M, e.a., Using gene expression profiling to identify a prognostic molecular spectrum in gliomas. Cancer Sci, 2009. 100: p. 8.

15. Verhaak, R.G., et al., Integrated genomic analysis identifies clinically relevant subtypes of glioblastoma characterized by abnormalities in PDGFRA, IDHI, EGFR, and NF1. Cancer Cell, 2010. 17(1): p. 98-110.

16. Wuchty S, e.a., Gene pathways and subnetworks distinguish between major glioma subtypes and elucidate potential underlying biology. Journal of Biomedical Informatics, 2006. 43: p. 8.

17. Markoa NF, T.S., Gene H. Barnett GH, Weil R Genomic expression patterns distinguish long-term from short-term glioblastoma survivors: A preliminary feasibility study. Genomics, 2003. 91: p. 12.

18. van den Boom J, e.a., Characterization of gene expression profiles associated with glioma progression using oligonucleotide-based microarray analysis and real-time reverse transcription-polymerase chain reaction. Am J Pathol 2003. 163: p. 11. 
19. Heyman M, e.a., Deletions of the short arm of chromosome 9, including the interferon-alpha/-beta genes, in acute lymphocytic leukemia. Studies on loss of heterozygosity, parental origin of deleted genes and prognosis. Int J Cancer, 1993. 54: p. 6.

20. Heyman, M., et al., Interferon system defects in malignant T-cells. Leukemia, 1994. 8(3): p. 425-34.

21. Fountain, J.W., et al., Homozygous deletions within human chromosome band 9p21 in melanoma. Proc Natl Acad Sci U S A, 1992. 89(21): p. 10557-61.

22. James CD, e.a., Chromosome 9 Deletion Mapping Reveals Interferon $\alpha$ and Interferon $\beta-1$ Gene Deletions in Human Glial Tumors. Cancer Res 1991. 51: p. 5.

23. Olopade O, e.a., Molecular Analysis of Deletions of the Short Arm of Chromosome 9 in Human Gliomas. Cancer Res 1992. 52: p. 7.

24. Bello MJ, e.a., Molecular and cytogenetic analysis of chromosome 9 deletions in 75 malignant gliomas. Genes Chromosomes Cancer 1994. 9: p. 9.

25. Serrano M, H.G., Beach D, A new regulatory motif in cell cycle control causing specific inhibition of cyclin D/CDK4. Nature, 1993. 366: p. 4.

26. Cairns, P., et al., Rates of p16 (MTS1) mutations in primary tumors with 9p loss. Science, 1994. 265(5170): p. 415-7.

27. Okamoto, A., et al., Mutations and altered expression of p16INK4 in human cancer. Proc Natl Acad Sci U S A, 1994. 91(23): p. 11045-9.

28. Hartwell, L., Defects in a cell cycle checkpoint may be responsible for the genomic instability of cancer cells. Cell, 1992. 71(4): p. 543-6.

29. He, J., J.J. Olson, and C.D. James, Lack of p16INK4 or retinoblastoma protein $(p R b)$, or amplification-associated overexpression of cdk4 is observed in distinct subsets of malignant glial tumors and cell lines. Cancer Res, 1995. 55(21): p. 4833-6.

30. Nishikawa, R., et al., Loss of P16INK4 expression is frequent in high grade gliomas. Cancer Res, 1995. 55(9): p. 1941-5.

31. Taha, M., et al., Extra-cranial metastasis of glioblastoma multiforme presenting as acute parotitis. Br J Neurosurg, 2005. 19(4): p. 348-51.

32. Rajagopalan, V., et al., Bone marrow metastases from glioblastoma multiforme--A case report and review of the literature. J Neurooncol, 2005. 72(2): p. 157-61.

33. Seo, Y.J., et al., Extraneural metastasis of glioblastoma multiforme presenting as an unusual neck mass. J Korean Neurosurg Soc, 2012. 51(3): p. 147-50.

34. Nagpal, S., The role of BCNU polymer wafers (Gliadel) in the treatment of malignant glioma. Neurosurg Clin N Am, 2012. 23(2): p. 289-95, ix.

35. Chamberlain, M.C., Treatment options for glioblastoma. Neurosurg Focus, 2006. 20(4): p. E19.

36. Allhenn, D., M.A. Boushehri, and A. Lamprecht, Drug delivery strategies for the treatment of malignant gliomas. Int J Pharm, 2012. 436(1-2): p. 299-310.

37. Mrugala, M.M., J.E. Adair, and H.P. Kiem, Outside the box--novel therapeutic strategies for glioblastoma. Cancer J, 2012. 18(1): p. 51-8.

38. Rich, J.N., et al., Phase II trial of gefitinib in recurrent glioblastoma. J Clin Oncol, 2004. 22(1): p. 133-42. 
39. Franceschi, E., et al., Gefitinib in patients with progressive high-grade gliomas: a multicentre phase II study by Gruppo Italiano Cooperativo di Neuro-Oncologia (GICNO). Br J Cancer, 2007. 96(7): p. 1047-51.

40. Prados, M.D., et al., Phase-1 trial of gefitinib and temozolomide in patients with malignant glioma: a North American brain tumor consortium study. Cancer Chemother Pharmacol, 2008. 61(6): p. 1059-67.

41. http://www.iressa.com.

42. Mok, T.S., et al., Gefitinib or carboplatin-paclitaxel in pulmonary adenocarcinoma. N Engl J Med, 2009. 361(10): p. 947-57.

43. Akhavan, D., T.F. Cloughesy, and P.S. Mischel, mTOR signaling in glioblastoma: lessons learned from bench to bedside. Neuro Oncol, 2010. 12(8): p. 882-9.

44. Salphati, L., et al., Targeting the PI3K pathway in the brain--efficacy of a PI3K inhibitor optimized to cross the blood-brain barrier. Clin Cancer Res, 2012. 18(22): p. 6239-48.

45. Thomas, A.A., M.S. Ernstoff, and C.E. Fadul, Immunotherapy for the treatment of glioblastoma. Cancer J, 2012. 18(1): p. 59-68.

46. D'Costa, S.M., et al., Vaccinia H5 is a multifunctional protein involved in viral DNA replication, postreplicative gene transcription, and virion morphogenesis. Virology, 2010. 401(1): p. 49-60.

47. http://clinicaltrials.gov/show/NCT00589875.

48. Pulkkanen, K.J. and S. Yla-Herttuala, Gene therapy for malignant glioma: current clinical status. Mol Ther, 2005. 12(4): p. 585-98.

49. Duarte, S., et al., Suicide gene therapy in cancer: where do we stand now? Cancer Lett, 2012. 324(2): p. 160-70.

50. http://clinicaltrials.gov/show/NCT01156584.

51. Tambuyzer, B.R., P. Ponsaerts, and E.J. Nouwen, Microglia: gatekeepers of central nervous system immunology. J Leukoc Biol, 2009. 85(3): p. 352-70.

52. Heimberger, A.B. and J.H. Sampson, Immunotherapy coming of age: what will it take to make it standard of care for glioblastoma? Neuro Oncol, 2011. 13(1): p. 3-13.

53. Hickey, W.F., B.L. Hsu, and H. Kimura, T-lymphocyte entry into the central nervous system. J Neurosci Res, 1991. 28(2): p. 254-60.

54. Cserr, H.F., C.J. Harling-Berg, and P.M. Knopf, Drainage of brain extracellular fluid into blood and deep cervical lymph and its immunological significance. Brain Pathol, 1992. 2(4): p. 269-76.

55. Jackson, C., et al., Challenges in immunotherapy presented by the glioblastoma multiforme microenvironment. Clin Dev Immunol, 2011. 2011: p. 732413.

56. Murphy, A.M. and S.D. Rabkin, Current status of gene therapy for brain tumors. Transl Res, 2013. 161(4): p. 339-54.

57. Parker, J.N., et al., Engineered herpes simplex virus expressing IL-12 in the treatment of experimental murine brain tumors. Proc Natl Acad Sci U S A, 2000. 97(5): p. 2208-13.

58. Ryu, C.H., et al., Gene therapy of intracranial glioma using interleukin 12secreting human umbilical cord blood-derived mesenchymal stem cells. Hum Gene Ther, 2011. 22(6): p. 733-43.

59. http://www.abedia.com/wiley/record_detail.php?ID=1552. 
60. Ohgaki, H., Genetic pathways to glioblastomas. Neuropathology, 2005. 25(1): p. $1-7$.

61. D'Avenia, P., et al., Tp53-gene transfer induces hypersensitivity to low doses of Xrays in glioblastoma cells: a strategy to convert a radio-resistant phenotype into a radiosensitive one. Cancer Lett, 2006. 231(1): p. 102-12.

62. Lang, F.F., et al., Phase I trial of adenovirus-mediated $p 53$ gene therapy for recurrent glioma: biological and clinical results. J Clin Oncol, 2003. 21(13): p. 2508-18.

63. Parker, J.N., et al., Oncolytic viral therapy of malignant glioma. Neurotherapeutics, 2009. 6(3): p. 558-69.

64. Vaha-Koskela, M.J., J.E. Heikkila, and A.E. Hinkkanen, Oncolytic viruses in cancer therapy. Cancer Lett, 2007. 254(2): p. 178-216.

65. Parato, K.A., et al., Recent progress in the battle between oncolytic viruses and tumours. Nat Rev Cancer, 2005. 5(12): p. 965-76.

66. Thorne, S.H., T. Hermiston, and D. Kirn, Oncolytic virotherapy: approaches to tumor targeting and enhancing antitumor effects. Semin Oncol, 2005. 32(6): p. 537-48.

67. Cattaneo, R., et al., Reprogrammed viruses as cancer therapeutics: targeted, armed and shielded. Nat Rev Microbiol, 2008. 6(7): p. 529-40.

68. Nguyen, T.L., et al., The emergence of combinatorial strategies in the development of RNA oncolytic virus therapies. Cell Microbiol, 2009. 11(6): p. 889-97.

69. Kelly, E. and S.J. Russell, History of oncolytic viruses: genesis to genetic engineering. Mol Ther, 2007. 15(4): p. 651-9.

70. Hammill, A.M., J. Conner, and T.P. Cripe, Oncolytic virotherapy reaches adolescence. Pediatr Blood Cancer, 2010. 55(7): p. 1253-63.

71. Dock, G., Rabies virus vaccination in a patient with cervical carcinoma. American Jouranl of Medical Sciences, 1904. 127: p. 563.

72. DePace, N., Sulla scomparsa di un enorme cancro vegetante del collo dell'utero senza cura chirurgica. Ginecologia, 1912. 9: p. 82-89.

73. Southam, C.M. and A.E. Moore, Clinical studies of viruses as antineoplastic agents with particular reference to Egypt 101 virus. Cancer, 1952. 5(5): p. 102534.

74. Huebner, R.J., et al., Studies on the use of viruses in the treatment of carcinoma of the cervix. Cancer, 1956. 9(6): p. 1211-8.

75. Zielinski, T. and E. Jordan, [Remote results of clinical observation of the oncolytic action of adenoviruses on cervix cancer]. Nowotwory, 1969. 19(3): p. 217-21.

76. Georgiades, J., et al., Research on the oncolytic effect of APC viruses in cancer of the cervix uteri; preliminary report. Biul Inst Med Morsk Gdansk, 1959. 10: p. 49-57.

77. Asada, T., Treatment of human cancer with mumps virus. Cancer, 1974. 34(6): p. 1907-28.

78. Hoster, H.A., R.P. Zanes, Jr., and E. Von Haam, Studies in Hodgkin's syndrome; the association of viral hepatitis and Hodgkin's disease; a preliminary report. Cancer Res, 1949. 9(8): p. 473-80. 
79. dos Reis, M., et al., Charting the host adaptation of influenza viruses. Mol Biol Evol, 2011. 28(6): p. 1755-67.

80. Lynch, J.P., 3rd, M. Fishbein, and M. Echavarria, Adenovirus. Semin Respir Crit Care Med, 2011. 32(4): p. 494-511.

81. de Gruijl, T.D. and R. van de Ven, Chapter six--Adenovirus-based immunotherapy of cancer: promises to keep. Adv Cancer Res, 2012. 115: p. 147220.

82. Steele, T.A., Recent developments in the virus therapy of cancer. Proc Soc Exp Biol Med, 2000. 223(2): p. 118-27.

83. Asadi-Moghaddam, K. and E.A. Chiocca, Gene- and viral-based therapies for brain tumors. Neurotherapeutics, 2009. 6(3): p. 547-57.

84. Ganly, I., et al., A phase I study of Onyx-015, an E1B attenuated adenovirus, administered intratumorally to patients with recurrent head and neck cancer. Clin Cancer Res, 2000. 6(3): p. 798-806.

85. Shen, Y., et al., Analyses of single-amino-acid substitution mutants of adenovirus type 5 E1B-55K protein. J Virol, 2001. 75(9): p. 4297-307.

86. Geoerger, B., et al., Oncolytic activity of the E1B-55 kDa-deleted adenovirus ONYX-015 is independent of cellular p53 status in human malignant glioma xenografts. Cancer Res, 2002. 62(3): p. 764-72.

87. Heise, C., et al., ONYX-015, an E1B gene-attenuated adenovirus, causes tumorspecific cytolysis and antitumoral efficacy that can be augmented by standard chemotherapeutic agents. Nat Med, 1997. 3(6): p. 639-45.

88. Fueyo, J., et al., Preclinical characterization of the antiglioma activity of a tropism-enhanced adenovirus targeted to the retinoblastoma pathway. J Natl Cancer Inst, 2003. 95(9): p. 652-60.

89. Lamfers, M.L., et al., Potential of the conditionally replicative adenovirus Ad5Delta24RGD in the treatment of malignant gliomas and its enhanced effect with radiotherapy. Cancer Res, 2002. 62(20): p. 5736-42.

90. http://clinicaltrials.gov/show/NCT01582516.

91. Chiocca, E.A., et al., A phase I open-label, dose-escalation, multi-institutional trial of injection with an E1B-Attenuated adenovirus, ONYX-015, into the peritumoral region of recurrent malignant gliomas, in the adjuvant setting. Mol Ther, 2004. 10(5): p. 958-66.

92. Kanai, R., et al., Effect of gamma34.5 deletions on oncolytic herpes simplex virus activity in brain tumors. J Virol, 2012. 86(8): p. 4420-31.

93. Cassady, K.A., M. Gross, and B. Roizman, The second-site mutation in the herpes simplex virus recombinants lacking the gamma134.5 genes precludes shutoff of protein synthesis by blocking the phosphorylation of eIF-2alpha. J Virol, 1998. 72(9): p. 7005-11.

94. Harrow, S., et al., HSV1716 injection into the brain adjacent to tumour following surgical resection of high-grade glioma: safety data and long-term survival. Gene Ther, 2004. 11(22): p. 1648-58.

95. http://www.virttu.com/clinical-studies

96. Sundaresan, P., et al., Attenuated, replication-competent herpes simplex virus type 1 mutant G207: safety evaluation in mice. J Virol, 2000. 74(8): p. 3832-41. 
97. Mineta, T., et al., Attenuated multi-mutated herpes simplex virus-1 for the treatment of malignant gliomas. Nat Med, 1995. 1(9): p. 938-43.

98. Mineta, T., S.D. Rabkin, and R.L. Martuza, Treatment of malignant gliomas using ganciclovir-hypersensitive, ribonucleotide reductase-deficient herpes simplex viral mutant. Cancer Res, 1994. 54(15): p. 3963-6.

99. Markert, J.M., et al., Phase Ib trial of mutant herpes simplex virus G207 inoculated pre-and post-tumor resection for recurrent GBM. Mol Ther, 2009. 17(1): p. 199-207.

100. http://clinicaltrials.gov/show/NCT00028158.

101. http://clinicaltrials.gov/show/NCT00157703.

102. Rhode, S.L., 3rd, Replication process of the parvovirus H-1. II. Isolation and characterization of H-1 replicative form DNA. J Virol, 1974. 13(2): p. 400-10.

103. Gerson SL, L.E., ed. Translational Approaches from Preclinical Studies to Clinical Implementation. 2nd ed. Parvovirus Vectors for the Gene Therapy of Cancer, ed. J.K.a.C.S. Wong. 2002, Academic Press. 53-71.

104. Rommelaere, J., et al., Oncolytic parvoviruses as cancer therapeutics. Cytokine Growth Factor Rev, 2010. 21(2-3): p. 185-95.

105. Di Piazza, M., et al., Cytosolic activation of cathepsins mediates parvovirus $H-1$ induced killing of cisplatin and TRAIL-resistant glioma cells. J Virol, 2007. 81(8): p. 4186-98.

106. Geletneky, K., et al., Regression of advanced rat and human gliomas by local or systemic treatment with oncolytic parvovirus $H-1$ in rat models. Neuro Oncol, 2010. 12(8): p. 804-14.

107. Geletneky, K., et al., Phase I/IIa study of intratumoral/intracerebral or intravenous/intracerebral administration of Parvovirus H-1 (ParvOryx) in patients with progressive primary or recurrent glioblastoma multiforme: ParvOryx01 protocol. BMC Cancer, 2012. 12: p. 99.

108. Elankumaran, S., et al., Type I interferon-sensitive recombinant newcastle disease virus for oncolytic virotherapy. J Virol, 2010. 84(8): p. 3835-44.

109. Freeman, A.I., et al., Phase I/II trial of intravenous NDV-HUJ oncolytic virus in recurrent glioblastoma multiforme. Mol Ther, 2006. 13(1): p. 221-8.

110. Fiola, C., et al., Tumor selective replication of Newcastle disease virus: association with defects of tumor cells in antiviral defence. Int J Cancer, 2006. 119(2): p. 328-38.

111. http://clinicaltrials.gov/show/NCT01174537.

112. Coffey, M.C., et al., Reovirus therapy of tumors with activated Ras pathway. Science, 1998. 282(5392): p. 1332-4.

113. Figova, K., J. Hrabeta, and T. Eckschlager, Reovirus - possible therapy of cancer. Neoplasma, 2006. 53(6): p. 457-62.

114. Yang, W.Q., et al., Efficacy and safety evaluation of human reovirus type 3 in immunocompetent animals: racine and nonhuman primates. Clin Cancer Res, 2004. 10(24): p. 8561-76.

115. Russell, S.J., RNA viruses as virotherapy agents. Cancer Gene Ther, 2002. 9(12): p. 961-6. 
116. Forsyth, P., et al., A phase I trial of intratumoral administration of reovirus in patients with histologically confirmed recurrent malignant gliomas. Mol Ther, 2008. 16(3): p. 627-32.

117. http://clinicaltrials.gov/show/NCT00528684.

118. Tatsuo, H., et al., SLAM (CDw150) is a cellular receptor for measles virus. Nature, 2000. 406(6798): p. 893-7.

119. Naniche, D., et al., Human membrane cofactor protein (CD46) acts as a cellular receptor for measles virus. J Virol, 1993. 67(10): p. 6025-32.

120. Galanis, E., Therapeutic potential of oncolytic measles virus: promises and challenges. Clin Pharmacol Ther, 2010. 88(5): p. 620-5.

121. Haralambieva, I., et al., Engineering oncolytic measles virus to circumvent the intracellular innate immune response. Mol Ther, 2007. 15(3): p. 588-97.

122. Myers, R., et al., Toxicology study of repeat intracerebral administration of a measles virus derivative producing carcinoembryonic antigen in rhesus macaques in support of a phase I/II clinical trial for patients with recurrent gliomas. Hum Gene Ther, 2008. 19(7): p. 690-8.

123. http://clinicaltrials.gov/show/NCT00390299.

124. Goetz, C., et al., Oncolytic poliovirus against malignant glioma. Future Virol, 2011. 6(9): p. 1045-1058.

125. Ochiai, H., et al., Treatment of intracerebral neoplasia and neoplastic meningitis with regional delivery of oncolytic recombinant poliovirus. Clin Cancer Res, 2004. 10(14): p. 4831-8.

126. de Breyne, S., et al., Direct functional interaction of initiation factor eIF4G with type 1 internal ribosomal entry sites. Proc Natl Acad Sci U S A, 2009. 106(23): p. 9197-202.

127. Goetz, C. and M. Gromeier, Preparing an oncolytic poliovirus recombinant for clinical application against glioblastoma multiforme. Cytokine Growth Factor Rev, 2010. 21(2-3): p. 197-203.

128. Gromeier, M., L. Alexander, and E. Wimmer, Internal ribosomal entry site substitution eliminates neurovirulence in intergeneric poliovirus recombinants. Proc Natl Acad Sci U S A, 1996. 93(6): p. 2370-5.

129. Gromeier, M., et al., Intergeneric poliovirus recombinants for the treatment of malignant glioma. Proc Natl Acad Sci U S A, 2000. 97(12): p. 6803-8.

130. Toyoda, H., et al., Oncolytic treatment and cure of neuroblastoma by a novel attenuated poliovirus in a novel poliovirus-susceptible animal model. Cancer Res, 2007. 67(6): p. 2857-64.

131. http://clinicaltrials.gov/show/NCT01491893.

132. Maetzig, T., et al., Gammaretroviral vectors: biology, technology and application. Viruses, 2011.3(6): p. 677-713.

133. Tai, C.K., et al., Single-shot, multicycle suicide gene therapy by replicationcompetent retrovirus vectors achieves long-term survival benefit in experimental glioma. Mol Ther, 2005. 12(5): p. 842-51.

134. Ostertag, D., et al., Brain tumor eradication and prolonged survival from intratumoral conversion of 5-fluorocytosine to 5-fluorouracil using a nonlytic retroviral replicating vector. Neuro Oncol, 2012. 14(2): p. 145-59. 
135. Perez, O.D., et al., Design and selection of Toca 511 for clinical use: modified retroviral replicating vector with improved stability and gene expression. Mol Ther, 2012. 20(9): p. 1689-98.

136. Rainov, N.G., A phase III clinical evaluation of herpes simplex virus type 1 thymidine kinase and ganciclovir gene therapy as an adjuvant to surgical resection and radiation in adults with previously untreated glioblastoma multiforme. Hum Gene Ther, 2000. 11(17): p. 2389-401.

137. http://clinicaltrials.gov/show/NCT01470794.

138. Zemp, F.J., et al., Oncolytic viruses as experimental treatments for malignant gliomas: using a scourge to treat a devil. Cytokine Growth Factor Rev, 2010. 21(2-3): p. 103-17.

139. Garber, K., China approves world's first oncolytic virus therapy for cancer treatment. J Natl Cancer Inst, 2006. 98(5): p. 298-300.

140. Miller, C.R., et al., Differential susceptibility of primary and established human glioma cells to adenovirus infection: targeting via the epidermal growth factor receptor achieves fiber receptor-independent gene transfer. Cancer Res, 1998. 58(24): p. 5738-48.

141. Li, Y., et al., Loss of adenoviral receptor expression in human bladder cancer cells: a potential impact on the efficacy of gene therapy. Cancer Res, 1999. 59(2): p. 325-30.

142. Barnett, B.G., C.J. Crews, and J.T. Douglas, Targeted adenoviral vectors. Biochim Biophys Acta, 2002. 1575(1-3): p. 1-14.

143. Zhang, Y. and J.M. Bergelson, Adenovirus receptors. J Virol, 2005. 79(19): p. 12125-31.

144. Arduino, P.G. and S.R. Porter, Herpes Simplex Virus Type 1 infection: overview on relevant clinico-pathological features. J Oral Pathol Med, 2008. 37(2): p. 10721.

145. Lu, Y., et al., Binding of the influenza virus NS1 protein to double-stranded RNA inhibits the activation of the protein kinase that phosphorylates the elF-2 translation initiation factor. Virology, 1995. 214(1): p. 222-8.

146. Markert, J.M., et al., Conditionally replicating herpes simplex virus mutant, G207 for the treatment of malignant glioma: results of a phase I trial. Gene Ther, 2000. 7(10): p. 867-74.

147. Bloom, M.E., et al., Analysis of parvovirus infections using strand-specific hybridization probes. Virus Res, 1989. 14(1): p. 1-25.

148. Maxwell, I.H., K.L. Terrell, and F. Maxwell, Autonomous parvovirus vectors. Methods, 2002. 28(2): p. 168-81.

149. Herrero, Y.C.M., et al., Parvovirus H-1 infection of human glioma cells leads to complete viral replication and efficient cell killing. Int J Cancer, 2004. 109(1): p. 76-84.

150. Seal, B.S., D.J. King, and H.S. Sellers, The avian response to Newcastle disease virus. Dev Comp Immunol, 2000. 24(2-3): p. 257-68.

151. Knipe, D.a.h.P., ed. Fields' Virology. 4th ed. Reoviruses and their replication, ed. N.M.a.S. LA. 2001, Lippincott-Raven Press: Philadelphia. 1679-1728.

152. Moss, W.J. and D.E. Griffin, Measles. Lancet, 2012. 379(9811): p. 153-64. 
153. Okwo-Bele, J.M. and T. Cherian, The expanded programme on immunization: a lasting legacy of smallpox eradication. Vaccine, 2011. 29 Suppl 4: p. D74-9.

154. Centers for Disease, C. and Prevention, Progress toward measles elimination-region of the Americas, 2002-2003. MMWR Morb Mortal Wkly Rep, 2004. 53(14): p. 304-6.

155. Noyce, R.S., et al., Tumor cell marker PVRL4 (nectin 4) is an epithelial cell receptor for measles virus. PLoS Pathog, 2011. 7(8): p. e1002240.

156. Noyce, R.S. and C.D. Richardson, Nectin 4 is the epithelial cell receptor for measles virus. Trends Microbiol, 2012. 20(9): p. 429-39.

157. Jurianz, K., et al., Complement resistance of tumor cells: basal and induced mechanisms. Mol Immunol, 1999. 36(13-14): p. 929-39.

158. Ulasov, I.V., et al., CD46 represents a target for adenoviral gene therapy of malignant glioma. Hum Gene Ther, 2006. 17(5): p. 556-64.

159. Fabre-Lafay, S., et al., Nectin-4 is a new histological and serological tumor associated marker for breast cancer. BMC Cancer, 2007. 7: p. 73.

160. Takano, A., et al., Identification of nectin-4 oncoprotein as a diagnostic and therapeutic target for lung cancer. Cancer Res, 2009. 69(16): p. 6694-703.

161. Derycke, M.S., et al., Nectin 4 overexpression in ovarian cancer tissues and serum: potential role as a serum biomarker. Am J Clin Pathol, 2010. 134(5): p. 835-45.

162. Koga, R., et al., Measles virus-induced immunosuppression in SLAM knock-in mice. J Virol, 2010. 84(10): p. 5360-7.

163. Aylward, B. and R. Tangermann, The global polio eradication initiative: lessons learned and prospects for success. Vaccine, 2011. 29 Suppl 4: p. D80-5.

164. Knipe, D., Howley PM, ed. Fields' Virology. 4 ed. Picornaviridae: the viruses and their replication, ed. R. VR. Vol. 1. 2001, Lippincott, WIlliams and Wilkins: Philadelphia. 685-772.

165. Masson, D., et al., Overexpression of the CD155 gene in human colorectal carcinoma. Gut, 2001. 49(2): p. 236-40.

166. Campbell, S.A., et al., Genetic determinants of cell type-specific poliovirus propagation in HEK 293 cells. J Virol, 2005. 79(10): p. 6281-90.

167. Neplioueva, V., et al., Tissue type-specific expression of the dsRNA-binding protein 76 and genome-wide elucidation of its target mRNAs. PLoS One, 2010. 5(7): p. e11710.

168. Goetz, C., et al., MAPK signal-integrating kinase controls cap-independent translation and cell type-specific cytotoxicity of an oncolytic poliovirus. Mol Ther, 2010. 18(11): p. 1937-46.

169. Horie, H., et al., Analysis of the accumulation of mutants in Sabin attenuated polio vaccine viruses passaged in Vero cells. Vaccine, 2001. 19(11-12): p. 14569.

170. Wimmer, E., C.U. Hellen, and X. Cao, Genetics of poliovirus. Annu Rev Genet, 1993. 27: p. 353-436.

171. Braoudaki, M. and F. Tzortzatou-Stathopoulou, Tumorigenesis related to retroviral infections. J Infect Dev Ctries, 2011. 5(11): p. 751-8. 
172. Howe, S.J., et al., Insertional mutagenesis combined with acquired somatic mutations causes leukemogenesis following gene therapy of SCID-X1 patients. J Clin Invest, 2008. 118(9): p. 3143-50.

173. Stein, S., et al., Genomic instability and myelodysplasia with monosomy 7 consequent to EVIl activation after gene therapy for chronic granulomatous disease. Nat Med, 2010. 16(2): p. 198-204.

174. Hacein-Bey-Abina, S., et al., Insertional oncogenesis in 4 patients after retrovirus-mediated gene therapy of $S C I D-X 1$. J Clin Invest, 2008. 118(9): $\mathrm{p}$. 3132-42.

175. Miller, D.G., M.A. Adam, and A.D. Miller, Gene transfer by retrovirus vectors occurs only in cells that are actively replicating at the time of infection. Mol Cell Biol, 1990. 10(8): p. 4239-42.

176. Hein, A., et al., Effects of adoptive immune transfers on murine leukemia virusinfection of rats. Virology, 1995. 211(2): p. 408-17.

177. Lun, X., et al., Myxoma virus virotherapy for glioma in immunocompetent animal models: optimizing administration routes and synergy with rapamycin. Cancer Res, 2010. 70(2): p. 598-608.

178. Wojton, J. and B. Kaur, Impact of tumor microenvironment on oncolytic viral therapy. Cytokine Growth Factor Rev, 2010. 21(2-3): p. 127-34.

179. Cheng, J., et al., Human matrix metalloproteinase-8 gene delivery increases the oncolytic activity of a replicating adenovirus. Mol Ther, 2007. 15(11): p. $1982-$ 90.

180. McKee, T.D., et al., Degradation of fibrillar collagen in a human melanoma xenograft improves the efficacy of an oncolytic herpes simplex virus vector. Cancer Res, 2006. 66(5): p. 2509-13.

181. Muldoon, L.L., et al., Chemotherapy delivery issues in central nervous system malignancy: a reality check. J Clin Oncol, 2007. 25(16): p. 2295-305.

182. Green, N.K. and L.W. Seymour, Adenoviral vectors: systemic delivery and tumor targeting. Cancer Gene Ther, 2002. 9(12): p. 1036-42.

183. Liu, R., R.L. Martuza, and S.D. Rabkin, Intracarotid delivery of oncolytic HSV vector G47Delta to metastatic breast cancer in the brain. Gene Ther, 2005. 12(8): p. 647-54.

184. Vlachaki, M.T., et al., Impact of preimmunization on adenoviral vector expression and toxicity in a subcutaneous mouse cancer model. Mol Ther, 2002. 6(3): p. 3428.

185. Miest, T.S., et al., Envelope-chimeric entry-targeted measles virus escapes neutralization and achieves oncolysis. Mol Ther, 2011. 19(10): p. 1813-20.

186. Lorence, R.M., et al., Complete regression of human neuroblastoma xenografts in athymic mice after local Newcastle disease virus therapy. J Natl Cancer Inst, 1994. 86(16): p. 1228-33.

187. Power, A.T., et al., Carrier cell-based delivery of an oncolytic virus circumvents antiviral immunity. Mol Ther, 2007. 15(1): p. 123-30.

188. Green, N.K., et al., Extended plasma circulation time and decreased toxicity of polymer-coated adenovirus. Gene Ther, 2004. 11(16): p. 1256-63. 
189. Sistigu, A., et al., Immunomodulatory effects of cyclophosphamide and implementations for vaccine design. Semin Immunopathol, 2011. 33(4): p. 36983.

190. Peng, K.W., et al., Using clinically approved cyclophosphamide regimens to control the humoral immune response to oncolytic viruses. Gene Ther, 2013. 20(3): p. 255-61.

191. Ikeda, K., et al., Oncolytic virus therapy of multiple tumors in the brain requires suppression of innate and elicited antiviral responses. Nat Med, 1999. 5(8): p. 881-7.

192. Qiao, J., et al., Cyclophosphamide facilitates antitumor efficacy against subcutaneous tumors following intravenous delivery of reovirus. Clin Cancer Res, 2008. 14(1): p. 259-69.

193. Alain, T., et al., Vesicular stomatitis virus oncolysis is potentiated by impairing mTORC1-dependent type I IFN production. Proc Natl Acad Sci U S A, 2010. 107(4): p. 1576-81.

194. Lun, X., et al., Efficacy and safety/toxicity study of recombinant vaccinia virus JX-594 in two immunocompetent animal models of glioma. Mol Ther, 2010. 18(11): p. 1927-36.

195. Cole, C., et al., Tumor-targeted, systemic delivery of therapeutic viral vectors using hitchhiking on antigen-specific T cells. Nat Med, 2005. 11(10): p. 1073-81.

196. Coukos, G., et al., Use of carrier cells to deliver a replication-selective herpes simplex virus-1 mutant for the intraperitoneal therapy of epithelial ovarian cancer. Clin Cancer Res, 1999. 5(6): p. 1523-37.

197. Raykov, Z., et al., Carrier cell-mediated delivery of oncolytic parvoviruses for targeting metastases. Int J Cancer, 2004. 109(5): p. 742-9.

198. Komarova, S., et al., Mesenchymal progenitor cells as cellular vehicles for delivery of oncolytic adenoviruses. Mol Cancer Ther, 2006. 5(3): p. 755-66.

199. Thaci, B., et al., Pharmacokinetic study of neural stem cell-based cell carrier for oncolytic virotherapy: targeted delivery of the therapeutic payload in an orthotopic brain tumor model. Cancer Gene Ther, 2012. 19(6): p. 431-42.

200. Platten, M., W. Wick, and M. Weller, Malignant glioma biology: role for TGFbeta in growth, motility, angiogenesis, and immune escape. Microsc Res Tech, 2001. 52(4): p. 401-10.

201. Grauer, O.M., et al., CD4+FoxP3+ regulatory T cells gradually accumulate in gliomas during tumor growth and efficiently suppress antiglioma immune responses in vivo. Int J Cancer, 2007. 121(1): p. 95-105.

202. Okada, H., et al., Immunotherapeutic approaches for glioma. Crit Rev Immunol, 2009. 29(1): p. 1-42.

203. Dunn, G.P., L.J. Old, and R.D. Schreiber, The immunobiology of cancer immunosurveillance and immunoediting. Immunity, 2004. 21(2): p. 137-48.

204. Sinkovics, J.G. and J.C. Horvath, Natural and genetically engineered viral agents for oncolysis and gene therapy of human cancers. Arch Immunol Ther Exp (Warsz), 2008. 56 Suppl 1: p. 3s-59s. 
205. Batliwalla, F.M., et al., A 15-year follow-up of AJCC stage III malignant melanoma patients treated postsurgically with Newcastle disease virus (NDV) oncolysate and determination of alterations in the CD8 T cell repertoire. Mol Med, 1998. 4(12): p. 783-94.

206. Steiner, H.H., et al., Antitumor vaccination of patients with glioblastoma multiforme: a pilot study to assess feasibility, safety, and clinical benefit. J Clin Oncol, 2004. 22(21): p. 4272-81.

207. Rose, J.K. and M.A. Whitt, Rhabdoviridae: The viruses and their replication, in Field's Virology, D. Knipe and P.M. Howley, Editors. 2001, Lippincott Williams \& Wilkins: Philadelphia. p. 1221-1244.

208. Kinnunen, P.M., et al., Epidemiology and host spectrum of Borna disease virus infections. J Gen Virol, 2013. 94(Pt 2): p. 247-62.

209. Bourhy, H., B. Kissi, and N. Tordo, Molecular diversity of the Lyssavirus genus. Virology, 1993. 194(1): p. 70-81.

210. Albertini, A.A., R.W. Ruigrok, and D. Blondel, Rabies virus transcription and replication. Adv Virus Res, 2011. 79: p. 1-22.

211. Hanson, R.P., Discussion of the natural history of vesicular stomatitis. Am J Epidemiol, 1968. 87(2): p. 264-6.

212. Rodriguez, L.L., Emergence and re-emergence of vesicular stomatitis in the United States. Virus Res, 2002. 85(2): p. 211-9.

213. Vernon, S.D., L.L. Rodriguez, and G.J. Letchworth, Vesicular stomatitis New Jersey virus glycoprotein gene sequence and neutralizing epitope stability in an enzootic focus. Virology, 1990. 177(1): p. 209-15.

214. Letchworth, G.J., L.L. Rodriguez, and J. Del cbarrera, Vesicular stomatitis. Vet J, 1999. 157(3): p. 239-60.

215. http://www.aphis.USDA.gov.

216. http://www.aphis.usda.gov/vs/nahss/equine/vsv/.

217. Howerth, E.W., et al., Experimental vesicular stomatitis in swine: effects of route of inoculation and steroid treatment. J Vet Diagn Invest, 1997. 9(2): p. 136-42.

218. Bruno-Lobo, M., et al., Pathogenesis of vesicular stomatitis virus infection in the infant hamster and mouse. An Microbiol (Rio J), 1968. 15: p. 53-68.

219. Cornish, T.E., et al., Pathogenesis of experimental vesicular stomatitis virus (New Jersey serotype) infection in the deer mouse (Peromyscus maniculatus). Vet Pathol, 2001. 38(4): p. 396-406.

220. Ozduman, K., et al., Peripheral immunization blocks lethal actions of vesicular stomatitis virus within the brain. J Virol, 2009. 83(22): p. 11540-9.

221. Wilks, C.R. and J.A. House, Susceptibility of various animals to the vesiculoviruses Isfahan and Chandipura. J Hyg (Lond), 1986. 97(2): p. 359-68.

222. Marriott, A.C., Complete genome sequences of Chandipura and Isfahan vesiculoviruses. Arch Virol, 2005. 150(4): p. 671-80.

223. Ogino, T. and A.K. Banerjee, An unconventional pathway of mRNA cap formation by vesiculoviruses. Virus Res, 2011. 162(1-2): p. 100-9.

224. Coll, J.M., Synthetic peptides from the heptad repeats of the glycoproteins of rabies, vesicular stomatitis and fish rhabdoviruses bind phosphatidylserine. Arch Virol, 1997. 142(10): p. 2089-97. 
225. Albertini, A.A., et al., Molecular and cellular aspects of rhabdovirus entry. Viruses, 2012. 4(1): p. 117-39.

226. Coil, D.A. and A.D. Miller, Phosphatidylserine is not the cell surface receptor for vesicular stomatitis virus. Journal of Virology, 2004. 78(20): p. 10920-6.

227. Bloor, S., et al., Endoplasmic reticulum chaperone gp96 is essential for infection with vesicular stomatitis virus. Proc Natl Acad Sci U S A, 2010. 107(15): p. 69705 .

228. Mire, C.E., J.M. White, and M.A. Whitt, A spatio-temporal analysis of matrix protein and nucleocapsid trafficking during vesicular stomatitis virus uncoating. PLoS Pathog, 2010. 6(7): p. e1000994.

229. Ge, P., et al., Cryo-EM model of the bullet-shaped vesicular stomatitis virus. Science, 2010. 327(5966): p. 689-93.

230. Pfeiffer, S., S.D. Fuller, and K. Simons, Intracellular sorting and basolateral appearance of the $G$ protein of vesicular stomatitis virus in Madin-Darby canine kidney cells. J Cell Biol, 1985. 101(2): p. 470-6.

231. Jayakar, H.R., E. Jeetendra, and M.A. Whitt, Rhabdovirus assembly and budding. Virus Research, 2004. 106(2): p. 117-132.

232. Brown, E.L. and D.S. Lyles, A novel method for analysis of membrane microdomains: vesicular stomatitis virus glycoprotein microdomains change in size during infection, and those outside of budding sites resemble sites of virus budding. Virology, 2003. 310(2): p. 343-58.

233. Bergmann, J.E. and P.J. Fusco, The M protein of vesicular stomatitis virus associates specifically with the basolateral membranes of polarized epithelial cells independently of the G protein. J Cell Biol, 1988. 107(5): p. 1707-15.

234. Pornillos, O., J.E. Garrus, and W.I. Sundquist, Mechanisms of enveloped RNA virus budding. Trends Cell Biol, 2002. 12(12): p. 569-79.

235. Hale, B.G., et al., The multifunctional NS1 protein of influenza A viruses. J Gen Virol, 2008. 89(Pt 10): p. 2359-76.

236. Blackford, A.N. and R.J. Grand, Adenovirus E1B 55-kilodalton protein: multiple roles in viral infection and cell transformation. J Virol, 2009. 83(9): p. 4000-12.

237. Jayakar, H.R. and M.A. Whitt, Identification of two additional translation products from the matrix $(M)$ gene that contribute to vesicular stomatitis virus cytopathology. J Virol, 2002. 76(16): p. 8011-8.

238. Gaudier, M., Y. Gaudin, and M. Knossow, Crystal structure of vesicular stomatitis virus matrix protein. EMBO J, 2002. 21(12): p. 2886-92.

239. Gomez-Puertas, P., et al., Influenza virus matrix protein is the major driving force in virus budding. J Virol, 2000. 74(24): p. 11538-47.

240. Timmins, J., et al., Vesicular release of ebola virus matrix protein VP40. Virology, 2001. 283(1): p. 1-6.

241. Jayakar, H.R., K.G. Murti, and M.A. Whitt, Mutations in the PPPY motif of vesicular stomatitis virus matrix protein reduce virus budding by inhibiting a late step in virion release. J Virol, 2000. 74(21): p. 9818-27.

242. Black, B.L., et al., The role of vesicular stomatitis virus matrix protein in inhibition of host-directed gene expression is genetically separable from its function in virus assembly. J Virol, 1993. 67(8): p. 4814-21. 
243. Connor, J.H., M.O. McKenzie, and D.S. Lyles, Role of residues 121 to 124 of vesicular stomatitis virus matrix protein in virus assembly and virus-host interaction. J Virol, 2006. 80(8): p. 3701-11.

244. Mire, C.E. and M.A. Whitt, The protease-sensitive loop of the vesicular stomatitis virus matrix protein is involved in virus assembly and protein translation. Virology, 2011. 416(1-2): p. 16-25.

245. Coulon, P., et al., Genetic evidence for multiple functions of the matrix protein of vesicular stomatitis virus. J Gen Virol, 1990. 71 ( Pt 4): p. 991-6.

246. Lyles, D.S., et al., Complementation of M gene mutants of vesicular stomatitis virus by plasmid-derived M protein converts spherical extracellular particles into native bullet shapes. Virology, 1996. 217(1): p. 76-87.

247. Justice, P.A., et al., Membrane vesiculation function and exocytosis of wild-type and mutant matrix proteins of vesicular stomatitis virus. J Virol, 1995. 69(5): p. 3156-60.

248. Harty, R.N., et al., A proline-rich motif within the matrix protein of vesicular stomatitis virus and rabies virus interacts with $W W$ domains of cellular proteins: implications for viral budding. J Virol, 1999. 73(4): p. 2921-9.

249. Flamand, A. and D.H. Bishop, Primary in vivo transcription of vesicular stomatitis virus and temperature-sensitive mutants of five vesicular stomatitis virus complementation groups. J Virol, 1973. 12(6): p. 1238-52.

250. Blondel, D., G.G. Harmison, and M. Schubert, Role of matrix protein in cytopathogenesis of vesicular stomatitis virus. J Virol, 1990. 64(4): p. 1716-25.

251. Melki, R., Y. Gaudin, and D. Blondel, Interaction between tubulin and the viral matrix protein of vesicular stomatitis virus: possible implications in the viral cytopathic effect. Virology, 1994. 202(1): p. 339-47.

252. Kopecky, S.A. and D.S. Lyles, The cell-rounding activity of the vesicular stomatitis virus matrix protein is due to the induction of cell death. J Virol, 2003. 77(9): p. 5524-8.

253. Lyles, D.S. and M.O. McKenzie, Activity of vesicular stomatitis virus M protein mutants in cell rounding is correlated with the ability to inhibit host gene expression and is not correlated with virus assembly function. Virology, 1997. 229(1): p. 77-89.

254. Black, B.L. and D.S. Lyles, Vesicular stomatitis virus matrix protein inhibits host cell-directed transcription of target genes in vivo. J Virol, 1992. 66(7): p. 405864.

255. Raux, H., et al., The matrix protein of vesicular stomatitis virus binds dynamin for efficient viral assembly. J Virol, 2010. 84(24): p. 12609-18.

256. Gu, C., et al., Direct dynamin-actin interactions regulate the actin cytoskeleton. EMBO J, 2010. 29(21): p. 3593-606.

257. Koyama, A.H., Induction of apoptotic DNA fragmentation by the infection of vesicular stomatitis virus. Virus Res, 1995. 37(3): p. 285-90.

258. Gadaleta, P., M. Vacotto, and F. Coulombie, Vesicular stomatitis virus induces apoptosis at early stages in the viral cycle and does not depend on virus replication. Virus Res, 2002. 86(1-2): p. 87-92. 
259. Kopecky, S.A., M.C. Willingham, and D.S. Lyles, Matrix protein and another viral component contribute to induction of apoptosis in cells infected with vesicular stomatitis virus. J Virol, 2001. 75(24): p. 12169-81.

260. Kurilla, M.G., H. Piwnica-Worms, and J.D. Keene, Rapid and transient localization of the leader RNA of vesicular stomatitis virus in the nuclei of infected cells. Proc Natl Acad Sci U S A, 1982. 79(17): p. 5240-4.

261. McGowan, J.J., S.U. Emerson, and R.R. Wagner, The plus-strand leader RNA of VSV inhibits DNA-dependent transcription of adenovirus and SV40 genes in a soluble whole-cell extract. Cell, 1982. 28(2): p. 325-33.

262. Kurilla, M.G. and J.D. Keene, The leader RNA of vesicular stomatitis virus is bound by a cellular protein reactive with anti-La lupus antibodies. Cell, 1983. 34(3): p. 837-45.

263. Dunigan, D.D., S. Baird, and J. Lucas-Lenard, Lack of correlation between the accumulation of plus-strand leader RNA and the inhibition of protein and RNA synthesis in vesicular stomatitis virus infected mouse L cells. Virology, 1986. 150(1): p. 231-46.

264. Clinton, G.M., et al., The matrix (M) protein of vesicular stomatitis virus regulates transcription. Cell, 1978. 15(4): p. 1455-62.

265. Lyles, D.S., L. Puddington, and B.J. McCreedy, Jr., Vesicular stomatitis virus $M$ protein in the nuclei of infected cells. J Virol, 1988. 62(11): p. 4387-92.

266. Ferran, M.C. and J.M. Lucas-Lenard, The vesicular stomatitis virus matrix protein inhibits transcription from the human beta interferon promoter. J Virol, 1997. 71(1): p. 371-7.

267. Weck, P.K. and R.R. Wagner, Inhibition of RNA synthesis in mouse myeloma cells infected with vesicular stomatitis virus. J Virol, 1978. 25(3): p. 770-80.

268. Ahmed, M. and D.S. Lyles, Effect of vesicular stomatitis virus matrix protein on transcription directed by host RNA polymerases I, II, and III. J Virol, 1998. 72(10): p. 8413-9.

269. Yuan, H., B.K. Yoza, and D.S. Lyles, Inhibition of host RNA polymerase IIdependent transcription by vesicular stomatitis virus results from inactivation of TFIID. Virology, 1998. 251(2): p. 383-92.

270. Yuan, H., S. Puckett, and D.S. Lyles, Inhibition of host transcription by vesicular stomatitis virus involves a novel mechanism that is independent of phosphorylation of TATA-binding protein (TBP) or association of TBP with TBPassociated factor subunits. J Virol, 2001. 75(9): p. 4453-8.

271. Connor, J.H. and D.S. Lyles, Inhibition of host and viral translation during vesicular stomatitis virus infection. eIF2 is responsible for the inhibition of viral but not host translation. J Biol Chem, 2005. 280(14): p. 13512-9.

272. Connor, J.H. and D.S. Lyles, Vesicular stomatitis virus infection alters the eIF4F translation initiation complex and causes dephosphorylation of the eIF4E binding protein 4E-BP1. J Virol, 2002. 76(20): p. 10177-87.

273. Whitlow, Z.W., J.H. Connor, and D.S. Lyles, New mRNAs are preferentially translated during vesicular stomatitis virus infection. J Virol, 2008. 82(5): p. 2286-94. 
274. Glodowski, D.R., J.M. Petersen, and J.E. Dahlberg, Complex nuclear localization signals in the matrix protein of vesicular stomatitis virus. J Biol Chem, 2002. 277(49): p. 46864-70.

275. von Kobbe, C., et al., Vesicular stomatitis virus matrix protein inhibits host cell gene expression by targeting the nucleoporin Nup98. Mol Cell, 2000. 6(5): p. 1243-52.

276. Enninga, J., et al., Role of nucleoporin induction in releasing an $m R N A$ nuclear export block. Science, 2002. 295(5559): p. 1523-5.

277. Petersen, J.M., L.S. Her, and J.E. Dahlberg, Multiple vesiculoviral matrix proteins inhibit both nuclear export and import. Proc Natl Acad Sci U S A, 2001. 98(15): p. 8590-5.

278. Faria, P.A., et al., VSV disrupts the Rael/mrnp41 mRNA nuclear export pathway. Mol Cell, 2005. 17(1): p. 93-102.

279. Rajani, K.R., et al., Complexes of vesicular stomatitis virus matrix protein with host Rael and Nup98 involved in inhibition of host transcription. PLoS Pathog, 2012. 8(9): p. e1002929.

280. Pauszek, S.J., R. Allende, and L.L. Rodriguez, Characterization of the full-length genomic sequences of vesicular stomatitis Cocal and Alagoas viruses. Arch Virol, 2008. 153(7): p. 1353-7.

281. Martin-Serrano, J., T. Zang, and P.D. Bieniasz, HIV-1 and Ebola virus encode small peptide motifs that recruit Tsg101 to sites of particle assembly to facilitate egress. Nat Med, 2001. 7(12): p. 1313-9.

282. Nagashima, S., et al., A PSAP motif in the ORF3 protein of hepatitis E virus is necessary for virion release from infected cells. J Gen Virol, 2011. 92(Pt 2): p. 269-78.

283. Obiang, L., et al., Phenotypes of vesicular stomatitis virus mutants with mutations in the PSAP motif of the matrix protein. J Gen Virol, 2012. 93(Pt 4): p. 857-65.

284. Irie, T., et al., Functional analysis of late-budding domain activity associated with the PSAP motif within the vesicular stomatitis virus M protein. J Virol, 2004. 78(14): p. 7823-7.

285. Irie, T., et al., Modifications of the PSAP region of the matrix protein lead to attenuation of vesicular stomatitis virus in vitro and in vivo. J Gen Virol, 2007. 88(Pt 9): p. 2559-67.

286. Irie, T., et al., Cytopathogenesis of vesicular stomatitis virus is regulated by the PSAP motif of M protein in a species-dependent manner. Viruses, 2012. 4(9): p. 1605-18.

287. Lun, X., et al., Effects of intravenously administered recombinant vesicular stomatitis virus (VSV(deltaM51)) on multifocal and invasive gliomas. J Natl Cancer Inst, 2006. 98(21): p. 1546-57.

288. Petersen, J.M., et al., The matrix protein of vesicular stomatitis virus inhibits nucleocytoplasmic transport when it is in the nucleus and associated with nuclear pore complexes. Mol Cell Biol, 2000. 20(22): p. 8590-601.

289. Stojdl, D.F., et al., VSV strains with defects in their ability to shutdown innate immunity are potent systemic anti-cancer agents. Cancer Cell, 2003. 4(4): p. 26375. 
290. Ahmed, M., et al., Ability of the matrix protein of vesicular stomatitis virus to suppress beta interferon gene expression is genetically correlated with the inhibition of host RNA and protein synthesis. J Virol, 2003. 77(8): p. 4646-57.

291. Lichty, B.D., et al., Vesicular stomatitis virus: re-inventing the bullet. Trends Mol Med, 2004. 10(5): p. 210-6.

292. Stojdl, D.F., et al., Exploiting tumor-specific defects in the interferon pathway with a previously unknown oncolytic virus. Nat Med, 2000. 6(7): p. 821-5.

293. Lichty, B.D., et al., Vesicular stomatitis virus: a potential therapeutic virus for the treatment of hematologic malignancy. Hum Gene Ther, 2004. 15(9): p. 821-31.

294. Balachandran, S. and G.N. Barber, Vesicular stomatitis virus (VSV) therapy of tumors. IUBMB Life, 2000. 50(2): p. 135-8.

295. Steinhoff, U., et al., Antiviral protection by vesicular stomatitis virus-specific antibodies in alpha/beta interferon receptor-deficient mice. J Virol, 1995. 69(4): p. 2153-8.

296. Stark, G.R., et al., How cells respond to interferons. Annu Rev Biochem, 1998. 67: p. 227-64.

297. Hardy, M.P., et al., Characterization of the type I interferon locus and identification of novel genes. Genomics, 2004. 84(2): p. 331-45.

298. Randall, R.E. and S. Goodbourn, Interferons and viruses: an interplay between induction, signalling, antiviral responses and virus countermeasures. J Gen Virol, 2008. 89(Pt 1): p. 1-47.

299. Theofilopoulos, A.N., et al., Type I interferons (alpha/beta) in immunity and autoimmunity. Annu Rev Immunol, 2005. 23: p. 307-36.

300. Takaoka, A., et al., Integration of interferon-alpha/beta signalling to p53 responses in tumour suppression and antiviral defence. Nature, 2003. 424(6948): p. 516-23.

301. Gerlach, N., et al., Anti-retroviral effects of type I IFN subtypes in vivo. Eur J Immunol, 2009. 39(1): p. 136-46.

302. Heiber, J.F. and G.N. Barber, Evaluation of innate immune signaling pathways in transformed cells. Methods Mol Biol, 2012. 797: p. 217-38.

303. Perry, A.K., et al., The host type I interferon response to viral and bacterial infections. Cell Res, 2005. 15(6): p. 407-22.

304. Lund, J.M., et al., Recognition of single-stranded RNA viruses by Toll-like receptor 7. Proc Natl Acad Sci U S A, 2004. 101(15): p. 5598-603.

305. Hornung, V., et al., 5'-Triphosphate RNA is the ligand for RIG-I. Science, 2006. 314(5801): p. 994-7.

306. Kato, H., et al., Cell type-specific involvement of RIG-I in antiviral response. Immunity, 2005. 23(1): p. 19-28.

307. de Veer, M.J., et al., Functional classification of interferon-stimulated genes identified using microarrays. J Leukoc Biol, 2001. 69(6): p. 912-20.

308. Schoggins, J.W., et al., A diverse range of gene products are effectors of the type I interferon antiviral response. Nature, 2011. 472(7344): p. 481-5.

309. Zhou, A., et al., Interferon action in triply deficient mice reveals the existence of alternative antiviral pathways. Virology, 1999. 258(2): p. 435-40.

310. Kroger, A., et al., Activities of IRF-1. J Interferon Cytokine Res, 2002. 22(1): p. 514. 
311. Stevens, A.M. and L.Y. Yu-Lee, The transcription factor interferon regulatory factor-1 is expressed during both early $G 1$ and the G1/S transition in the prolactin-induced lymphocyte cell cycle. Mol Endocrinol, 1992. 6(12): p. 223643.

312. Kirchhoff, S., F. Schaper, and H. Hauser, Interferon regulatory factor 1 (IRF-1) mediates cell growth inhibition by transactivation of downstream target genes. Nucleic Acids Res, 1993. 21(12): p. 2881-9.

313. Fujita, T., et al., Induction of endogenous IFN-alpha and IFN-beta genes by a regulatory transcription factor, IRF-1. Nature, 1989. 337(6204): p. 270-2.

314. Tamura, T., et al., The IRF family transcription factors in immunity and oncogenesis. Annu Rev Immunol, 2008. 26: p. 535-84.

315. Brien, J.D., et al., Interferon regulatory factor-1 (IRF-1) shapes both innate and CD8(+) T cell immune responses against West Nile virus infection. PLoS Pathog, 2011. 7(9): p. e1002230.

316. Haller, O. and G. Kochs, Interferon-induced mx proteins: dynamin-like GTPases with antiviral activity. Traffic, 2002. 3(10): p. 710-7.

317. Staeheli, P. and J. Pavlovic, Inhibition of vesicular stomatitis virus $m R N A$ synthesis by human MxA protein. J Virol, 1991. 65(8): p. 4498-501.

318. Stertz, S., et al., The antiviral potential of interferon-induced cotton rat Mx proteins against orthomyxovirus (influenza), rhabdovirus, and bunyavirus. J Interferon Cytokine Res, 2007. 27(10): p. 847-55.

319. Olszewski, M.A., J. Gray, and D.J. Vestal, In silico genomic analysis of the human and murine guanylate-binding protein $(G B P)$ gene clusters. J Interferon Cytokine Res, 2006. 26(5): p. 328-52.

320. Kresse, A., et al., Analyses of murine GBP homology clusters based on in silico, in vitro and in vivo studies. BMC Genomics, 2008. 9: p. 158.

321. Carter, C.C., V.Y. Gorbacheva, and D.J. Vestal, Inhibition of VSV and EMCV replication by the interferon-induced GTPase, $m G B P$-2: differential requirement for wild-type GTP binding domain. Arch Virol, 2005. 150(6): p. 1213-20.

322. MacMicking, J.D., IFN-inducible GTPases and immunity to intracellular pathogens. Trends Immunol, 2004. 25(11): p. 601-9.

323. Anderson, S.L., et al., Interferon-induced guanylate binding protein-1 (GBP-1) mediates an antiviral effect against vesicular stomatitis virus and encephalomyocarditis virus. Virology, 1999. 256(1): p. 8-14.

324. Degrandi, D., et al., Extensive characterization of IFN-induced GTPases mGBP1 to $\mathrm{mGBP} 10$ involved in host defense. J Immunol, 2007. 179(11): p. 7729-40.

325. Li, M., et al., Guanylate binding protein 1 is a novel effector of EGFR-driven invasion in glioblastoma. J Exp Med, 2011. 208(13): p. 2657-73.

326. Dong, B., et al., Phospholipid scramblase 1 potentiates the antiviral activity of interferon. J Virol, 2004. 78(17): p. 8983-93.

327. Zhou, Q., et al., Molecular cloning of human plasma membrane phospholipid scramblase. A protein mediating transbilayer movement of plasma membrane phospholipids. J Biol Chem, 1997. 272(29): p. 18240-4.

328. Sims, P.J. and T. Wiedmer, Unraveling the mysteries of phospholipid scrambling. Thromb Haemost, 2001. 86(1): p. 266-75. 
329. Sahu, S.K., et al., Phospholipid scramblases: an overview. Arch Biochem Biophys, 2007. 462(1): p. 103-14.

330. Chawla-Sarkar, M., et al., Apoptosis and interferons: role of interferon-stimulated genes as mediators of apoptosis. Apoptosis, 2003. 8(3): p. 237-49.

331. Sgorbissa, A., et al., Type I IFNs signaling and apoptosis resistance in glioblastoma cells. Apoptosis, 2011. 16(12): p. 1229-44.

332. Haybaeck, J., et al., STAT-1 expression in human glioblastoma and peritumoral tissue. Anticancer Res, 2007. 27(6B): p. 3829-35.

333. Du, Z., et al., Inhibition of type I interferon-mediated antiviral action in human glioma cells by the IKK inhibitors BMS-345541 and TPCA-1. J Interferon Cytokine Res, 2012. 32(8): p. 368-77.

334. Yoshino, A., et al., Therapeutic implications of interferon regulatory factor (IRF)-1 and IRF-2 in diffusely infiltrating astrocytomas (DIA): response to interferon (IFN)-beta in glioblastoma cells and prognostic value for DIA. J Neurooncol, 2005. 74(3): p. 249-60.

335. Versteeg, G.A. and A. Garcia-Sastre, Viral tricks to grid-lock the type I interferon system. Curr Opin Microbiol, 2010. 13(4): p. 508-16.

336. Andrejeva, J., et al., The V proteins of paramyxoviruses bind the IFN-inducible RNA helicase, mda-5, and inhibit its activation of the IFN-beta promoter. Proc Natl Acad Sci U S A, 2004. 101(49): p. 17264-9.

337. Kubota, T., et al., Mumps virus V protein antagonizes interferon without the complete degradation of STAT1. J Virol, 2005. 79(7): p. 4451-9.

338. Fan, L., T. Briese, and W.I. Lipkin, Z proteins of New World arenaviruses bind RIG-I and interfere with type I interferon induction. J Virol, 2010. 84(4): p. 178591.

339. Basu, D., et al., Novel influenza virus NS1 antagonists block replication and restore innate immune function. J Virol, 2009. 83(4): p. 1881-91.

340. Kamitani, W., et al., Severe acute respiratory syndrome coronavirus nsp 1 protein suppresses host gene expression by promoting host $m R N A$ degradation. Proc Natl Acad Sci U S A, 2006. 103(34): p. 12885-90.

341. Kopecky-Bromberg, S.A., et al., Severe acute respiratory syndrome coronavirus open reading frame $(O R F) 3 b, O R F$, and nucleocapsid proteins function as interferon antagonists. J Virol, 2007. 81(2): p. 548-57.

342. Brzozka, K., S. Finke, and K.K. Conzelmann, Identification of the rabies virus alpha/beta interferon antagonist: phosphoprotein $P$ interferes with phosphorylation of interferon regulatory factor 3. J Virol, 2005. 79(12): p. 767381.

343. An, H.Y., et al., Genetically modified VSV(NJ) vector is capable of accommodating a large foreign gene insert and allows high level gene expression. Virus Res, 2013. 171(1): p. 168-77.

344. Obuchi, M., M. Fernandez, and G.N. Barber, Development of recombinant vesicular stomatitis viruses that exploit defects in host defense to augment specific oncolytic activity. J Virol, 2003. 77(16): p. 8843-56.

345. Diaz, R.M., et al., Oncolytic immunovirotherapy for melanoma using vesicular stomatitis virus. Cancer Res, 2007. 67(6): p. 2840-8. 
346. van den Pol, A.N., K.P. Dalton, and J.K. Rose, Relative neurotropism of a recombinant rhabdovirus expressing a green fluorescent envelope glycoprotein. J Virol, 2002. 76(3): p. 1309-27.

347. Steinhauer, D.A. and J.J. Holland, Direct method for quantitation of extreme polymerase error frequencies at selected single base sites in viral RNA. J Virol, 1986. 57(1): p. 219-28.

348. Jackson, A.C., Rabies. Neurol Clin, 2008. 26(3): p. 717-26, ix.

349. Kennedy, P.G., Viral encephalitis. J Neurol, 2005. 252(3): p. 268-72.

350. Schneider-Schaulies, J., et al., Measles virus in the CNS: the role of viral and host factors for the establishment and maintenance of a persistent infection. $\mathrm{J}$

Neurovirol, 1999. 5(6): p. 613-22.

351. Dal Canto, M.C. and S.G. Rabinowitz, Murine central nervous system infection by a viral temperature-sensitive mutant: a subacute disease leading to demyelination. Am J Pathol, 1981. 102(3): p. 412-26.

352. Thomsen, A.R., et al., Cooperation of $B$ cells and $T$ cells is required for survival of mice infected with vesicular stomatitis virus. Int Immunol, 1997. 9(11): p. 1757-66.

353. Wollmann, G., P. Tattersall, and A.N. van den Pol, Targeting human glioblastoma cells: comparison of nine viruses with oncolytic potential. J Virol, 2005. 79(10): p. 6005-22.

354. Duntsch, C.D., et al., Recombinant vesicular stomatitis virus vectors as oncolytic agents in the treatment of high-grade gliomas in an organotypic brain tissue slice-glioma coculture model. J Neurosurg, 2004. 100(6): p. 1049-59.

355. Wollmann, G., et al., Some attenuated variants of vesicular stomatitis virus show enhanced oncolytic activity against human glioblastoma cells relative to normal brain cells. J Virol, 2010. 84(3): p. 1563-73.

356. Francoeur, A.M., L. Poliquin, and C.P. Stanners, The isolation of interferoninducing mutants of vesicular stomatitis virus with altered viral $P$ function for the inhibition of total protein synthesis. Virology, 1987. 160(1): p. 236-45.

357. Desforges, M., et al., Different host-cell shutoff strategies related to the matrix protein lead to persistence of vesicular stomatitis virus mutants on fibroblast cells. Virus Res, 2001. 76(1): p. 87-102.

358. Ahmed, M., S.D. Cramer, and D.S. Lyles, Sensitivity of prostate tumors to wild type and M protein mutant vesicular stomatitis viruses. Virology, 2004. 330(1): p. 34-49.

359. Cary, Z.D., M.C. Willingham, and D.S. Lyles, Oncolytic vesicular stomatitis virus induces apoptosis in U87 glioblastoma cells by a type II death receptor mechanism and induces cell death and tumor clearance in vivo. J Virol, 2011. 85(12): p. 5708-17.

360. Ozduman, K., et al., Systemic vesicular stomatitis virus selectively destroys multifocal glioma and metastatic carcinoma in brain. J Neurosci, 2008. 28(8): p. 1882-93.

361. Jenks, N., et al., Safety studies on intrahepatic or intratumoral injection of oncolytic vesicular stomatitis virus expressing interferon-beta in rodents and nonhuman primates. Hum Gene Ther, 2010. 21(4): p. 451-62. 
362. Naik, S., et al., Potent systemic therapy of multiple myeloma utilizing oncolytic vesicular stomatitis virus coding for interferon-beta. Cancer Gene Ther, 2012. 19(7): p. 443-50.

363. http://clinicaltrials.gov/show/NCT01628640.

364. Guertin, D.A. and D.M. Sabatini, Defining the role of mTOR in cancer. Cancer Cell, 2007. 12(1): p. 9-22.

365. Costa-Mattioli, M. and N. Sonenberg, RAPping production of type I interferon in pDCs through mTOR. Nat Immunol, 2008. 9(10): p. 1097-9.

366. Jayakar, H.R., VSV M protein domains involved in assembly and cytopathogenesis, in Microbiology2001, University of Tennessee Health Science Center: Memphis, TN. p. 216.

367. Familletti, P.C., S. Rubinstein, and S. Pestka, A convenient and rapid cytopathic effect inhibition assay for interferon. Methods Enzymol, 1981. 78(Pt A): p. 38794.

368. Sharma, A.K., et al., Development of a percutaneous cerebrospinal fluid collection technique in F-344 rats and evaluation of cell counts and total protein concentrations. Toxicol Pathol, 2006. 34(4): p. 393-5.

369. Ahmed, M., S. Puckett, and D.S. Lyles, Susceptibility of breast cancer cells to an oncolytic matrix $(M)$ protein mutant of vesicular stomatitis virus. Cancer Gene Ther, 2010. 17(12): p. 883-92.

370. Vogel, C. and E.M. Marcotte, Insights into the regulation of protein abundance from proteomic and transcriptomic analyses. Nat Rev Genet, 2012. 13(4): p. 22732.

371. Wollmann, G., M.D. Robek, and A.N. van den Pol, Variable deficiencies in the interferon response enhance susceptibility to vesicular stomatitis virus oncolytic actions in glioblastoma cells but not in normal human glial cells. J Virol, 2007. 81(3): p. 1479-91.

372. Furr, S.R., et al., RIG-I mediates nonsegmented negative-sense RNA virusinduced inflammatory immune responses of primary human astrocytes. Glia, 2010. 58(13): p. 1620-9.

373. Onoguchi, K., M. Yoneyama, and T. Fujita, Retinoic acid-inducible gene-I-like receptors. J Interferon Cytokine Res, 2011. 31(1): p. 27-31.

374. Marie, I., J.E. Durbin, and D.E. Levy, Differential viral induction of distinct interferon-alpha genes by positive feedback through interferon regulatory factor7. EMBO J, 1998. 17(22): p. 6660-9.

375. $\mathrm{Bi}, \mathrm{Z}$., et al., Vesicular stomatitis virus infection of the central nervous system activates both innate and acquired immunity. J Virol, 1995. 69(10): p. 6466-72.

376. Barth, R.F. and B. Kaur, Rat brain tumor models in experimental neuro-oncology: the C6, 9L, T9, RG2, F98, BT4C, RT-2 and CNS-1 gliomas. J Neurooncol, 2009. 94(3): p. 299-312.

377. Parsa, A.T., et al., Limitations of the C6/Wistar rat intracerebral glioma model: implications for evaluating immunotherapy. Neurosurgery, 2000. 47(4): p. 993-9; discussion 999-1000.

378. Clendenon, N.R., et al., Boron neutron capture therapy of a rat glioma. Neurosurgery, 1990. 26(1): p. 47-55. 
379. Barth, R.F., Rat brain tumor models in experimental neuro-oncology: the 9L, C6, T9, F98, RG2 (D74), RT-2 and CNS-1 gliomas. J Neurooncol, 1998. 36(1): p. 91102.

380. http://www.atcc.org/products/all/CRL-2397.

381. Nieder, C., et al., Treatment of unresectable glioblastoma multiforme. Anticancer Res, 2005. 25(6C): p. 4605-10.

382. Liu, Q., et al., Molecular properties of CD133+ glioblastoma stem cells derived from treatment-refractory recurrent brain tumors. J Neurooncol, 2009. 94(1): p. $1-19$.

383. Wollmann, G., K. Ozduman, and A.N. van den Pol, Oncolytic virus therapy for glioblastoma multiforme: concepts and candidates. Cancer J, 2012. 18(1): p. 6981 .

384. Auffinger, B., A.U. Ahmed, and M.S. Lesniak, Oncolytic virotherapy for malignant glioma: translating laboratory insights into clinical practice. Front Oncol, 2013. 3: p. 32.

385. Bergman, I., et al., Treatment of implanted mammary tumors with recombinant vesicular stomatitis virus targeted to Her2/neu. Int J Cancer, 2007. 121(2): p. 425-30.

386. Wollmann, G., et al., Vesicular stomatitis virus variants selectively infect and kill human melanomas but not normal melanocytes. J Virol, 2013. 87(12): p. 6644-59.

387. Johnsen, I.B., et al., Toll-like receptor 3-elicited MAPK activation induces stabilization of interferon-beta mRNA. Cytokine, 2012. 57(3): p. 337-46.

388. Norton, W.T., et al., Quantitative aspects of reactive gliosis: a review. Neurochem Res, 1992. 17(9): p. 877-85.

389. Kawano, H., et al., Role of the lesion scar in the response to damage and repair of the central nervous system. Cell Tissue Res, 2012. 349(1): p. 169-80.

390. Gack, M.U., et al., Roles of RIG-I N-terminal tandem CARD and splice variant in TRIM25-mediated antiviral signal transduction. Proc Natl Acad Sci U S A, 2008. 105(43): p. 16743-8.

391. Eisenacher, K. and A. Krug, Regulation of RLR-mediated innate immune signaling--it is all about keeping the balance. Eur J Cell Biol, 2012. 91(1): p. 3647.

392. Stirnweiss, A., et al., IFN regulatory factor-1 bypasses IFN-mediated antiviral effects through viperin gene induction. J Immunol, 2010. 184(9): p. 5179-85.

393. Maatta, A.M., et al., Evaluation of cancer virotherapy with attenuated replicative Semliki forest virus in different rodent tumor models. Int J Cancer, 2007. 121(4): p. 863-70.

394. Vaha-Koskela, M.J., et al., Resistance to two heterologous neurotropic oncolytic viruses, Semliki Forest virus and vaccinia virus, in experimental glioma. J Virol, 2013. 87(4): p. 2363-6.

395. Pinschewer, D.D., et al., Kinetics of protective antibodies are determined by the viral surface antigen. J Clin Invest, 2004. 114(7): p. 988-93.

396. Kurozumi, K., et al., Effect of tumor microenvironment modulation on the efficacy of oncolytic virus therapy. J Natl Cancer Inst, 2007. 99(23): p. 1768-81. 
397. Charan, S., H. Hengartner, and R.M. Zinkernagel, Antibodies against the two serotypes of vesicular stomatitis virus measured by enzyme-linked immunosorbent assay: immunodominance of serotype-specific determinants and induction of asymmetrically cross-reactive antibodies. J Virol, 1987. 61(8): p. 2509-14.

398. Prestwich, R.J., et al., The case of oncolytic viruses versus the immune system: waiting on the judgment of Solomon. Hum Gene Ther, 2009. 20(10): p. 1119-32.

399. Raykov, Z. and J. Rommelaere, Potential of tumour cells for delivering oncolytic viruses. Gene Ther, 2008. 15(10): p. 704-10.

400. Guo, Z.S., et al., The combination of immunosuppression and carrier cells significantly enhances the efficacy of oncolytic poxvirus in the pre-immunized host. Gene Ther, 2010. 17(12): p. 1465-75.

401. Ruotsalainen, J., et al., Interferon-beta sensitivity of tumor cells correlates with poor response to VA7 virotherapy in mouse glioma models. Mol Ther, 2012. 20(8): p. 1529-39. 


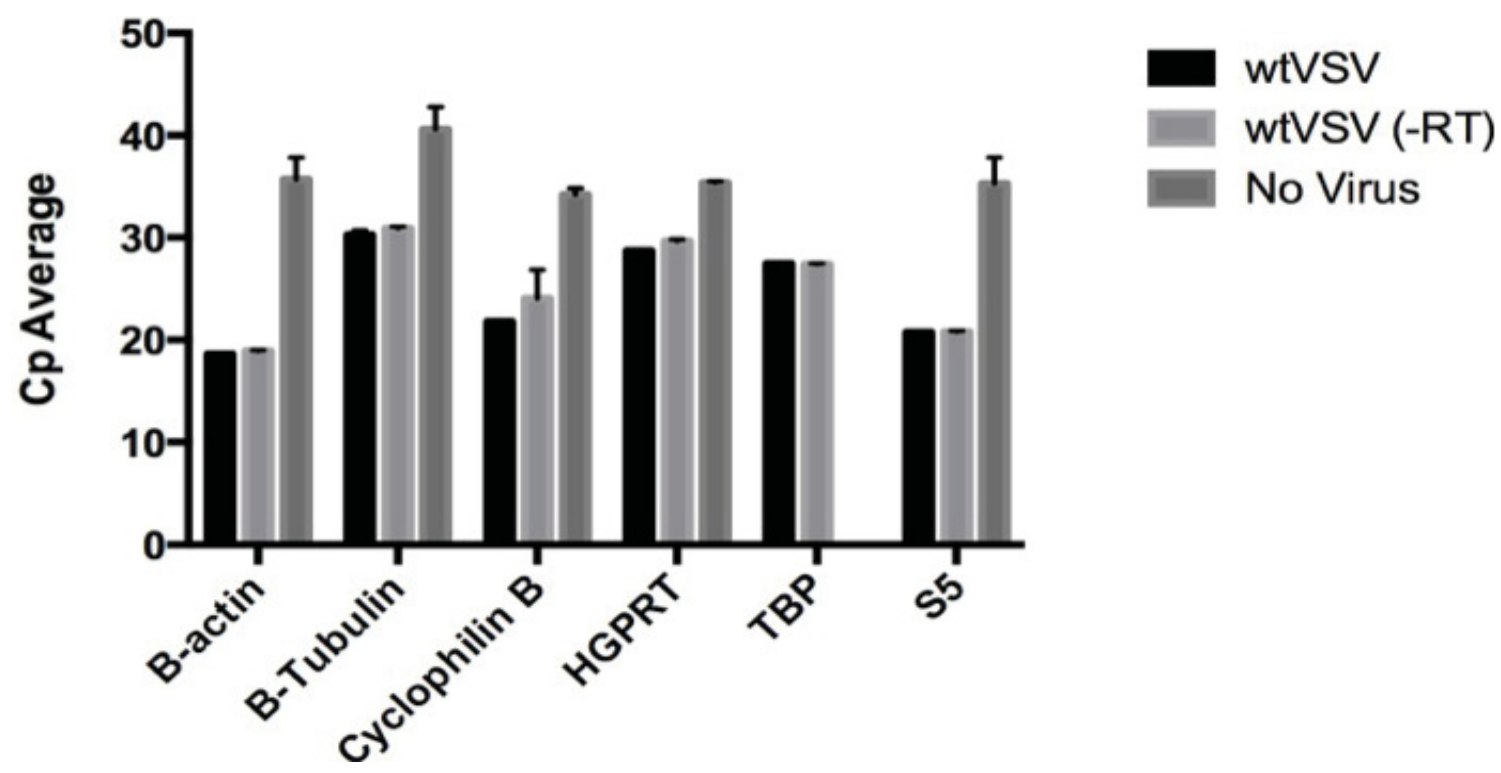

Figure A-1. Expression of housekeeping genes in FR cells

The expression of several housekeeping genes was tested in order to determine the best gene for normalization of interferon stimulated genes studies. FR cells were transfected with pI:C. At 24 hours post-treatment, cells were harvested and total RNA was extracted using Trizol reagent. cDNA was synthesized using M-MLV reverse transcriptase (Invitrogen) and random hexamers as primer. Quantitative PCR was performed on the LightCycler 480 (Molecular Resource Center, UTHSC) using Universal Probe Library (UPL) protocol for rat gene expression (Roche) (Table A-1). Universal probes are specific for the detection of the rat transcriptome and are labeled with fluorescein at the 5' end and a dark quencher dye at the 3'end that allow detection by standard SYBR Green I filters. The LightCycler 480 Taqman90 protocol was used which is preprogrammed to perform the following cycles: activation of DNA polymerase for 5 minutes at $95^{\circ} \mathrm{C}$; amplification of cDNA for 40 cycles which includes 10 seconds at $95^{\circ} \mathrm{C}$ denature, 20 seconds at $60^{\circ} \mathrm{C}$ annealing, and 10 seconds at $72^{\circ} \mathrm{C}$ extension; cool down for 30 seconds at $40^{\circ} \mathrm{C}$. From these results, $\beta$-actin was chosen as the housekeeping gene to use in further experiments. Each experiment was done in duplicate and results are expressed as the mean $\pm \mathrm{SD}$. 
Table A-1. qRT-PCR UPL primers for housekeeping genes

\begin{tabular}{|c|c|c|c|}
\hline Gene & Left Primer & Right Primer & $\begin{array}{c}\text { UPL } \\
\text { Probe \# }\end{array}$ \\
\hline Cyclophilin B & Acgtggttttcggcaaagt & Cttggtgttctccaccttcc & 97 \\
\hline$\beta$-actin & Cccgcgagtacaaccttct & cgtcatccatggcgaact & 17 \\
\hline $\begin{array}{l}\text { HPRT1 (Hypoxanthine } \\
\text { phosphoribosyl- } \\
\text { transferase 1) }\end{array}$ & Gaccggttctgtcatgtcg & acctggttcatcatcactaatcac & 95 \\
\hline$\beta$-tubulin & Cagagccattctggtggac & Gccagcaccactctgacc & 116 \\
\hline $\begin{array}{l}\text { TBP (TATA Box binding } \\
\text { protein) }\end{array}$ & $\begin{array}{l}\text { Cccaccagcagttcagtag } \\
\text { c }\end{array}$ & Cattctgggtttgatcattctg & 129 \\
\hline S5 (ribosomal protein S5) & Gactgagaagcccggtttg & cttgatgtccggggtctct & 5 \\
\hline
\end{tabular}




\section{Cyclophilin B}

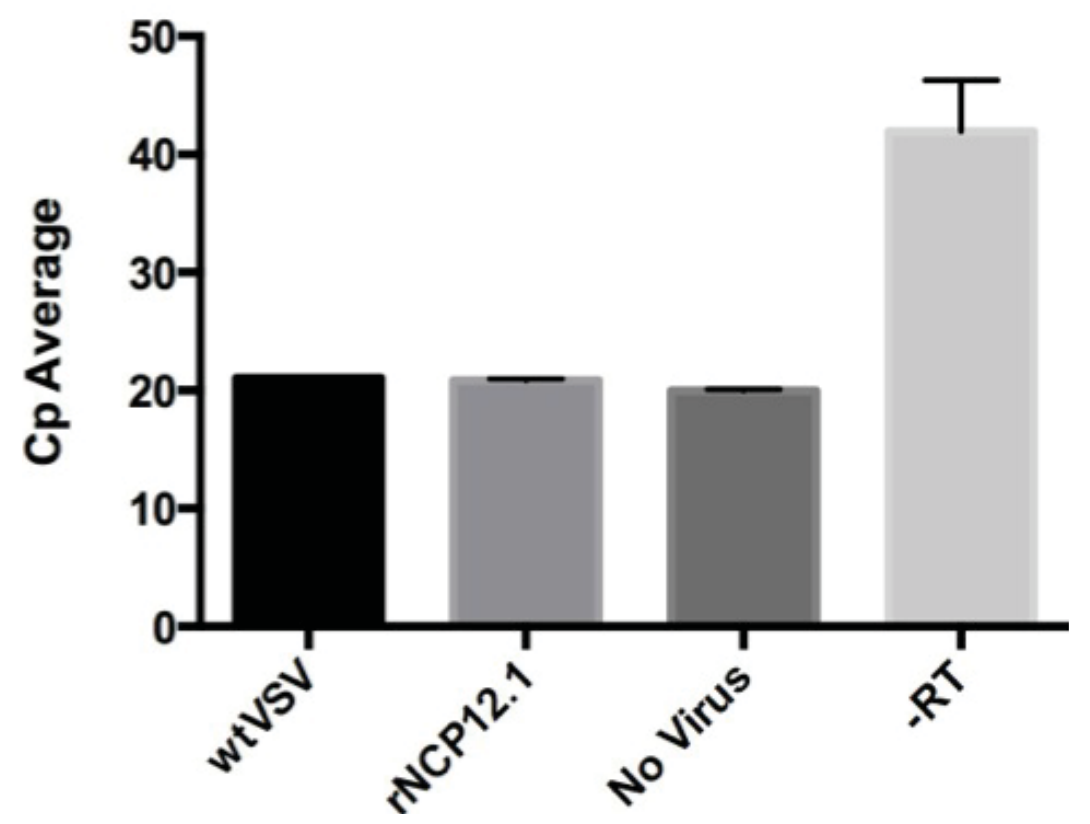

Figure A-2. Expression of Cyclophilin B in infected FR cells

Due to results observed following infection in C6 cells discussed in Chapter 3, Cyclophin $\mathrm{B}$ was chosen for further testing to determine the appropriate housekeeping gene for subsequent qRT-PCR studies. FR cells were infected with either wtVSV or rNCP12.1GFP at an MOI of 10 for one hour at $31^{\circ} \mathrm{C}$. At $24 \mathrm{hpi}$, cells were harvested and qRTPCR was performed as describe on the LightCycler 480 (Molecular Resource Center, UTHSC) using Universal Primary Library (UPL) protocol for rat gene expression (Roche) (Table A-1). Expression levels of Cyclophilin B were consistent despite infection. (-)RT controls demonstrated high $\mathrm{Cp}$ values as expected for samples not contaminated with chromosomal DNA. Therefore, Cyclophilin B was determined to be the most appropriate housekeeping gene for use in further qRT-PCR studies. Each experiment was done in duplicate and results are expressed as the mean $\pm \mathrm{SD}$ 


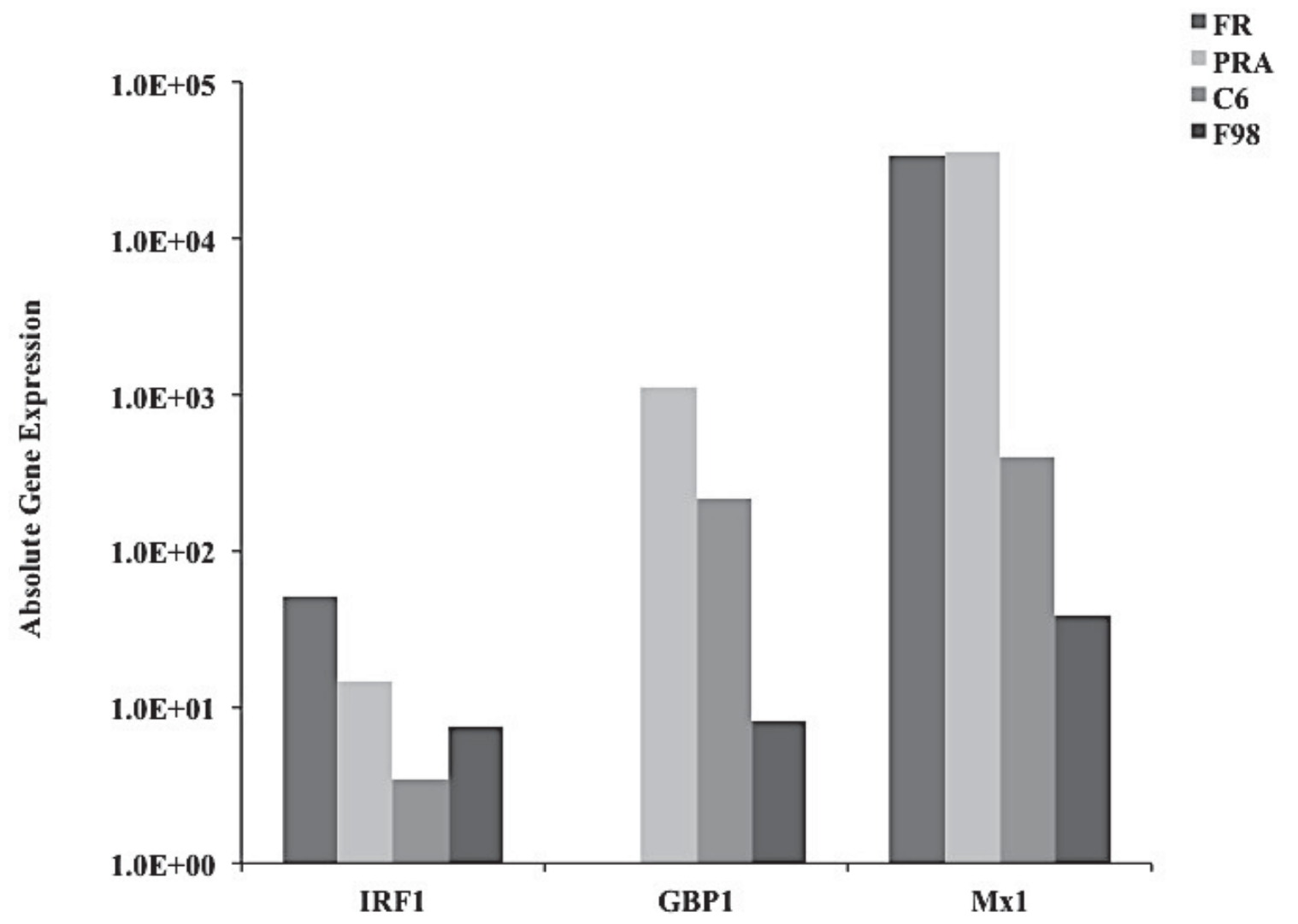

Figure A-3. Test of UPL primer sets for the expression of ISGs in multiple cell lines

Non-tumor (PRA) and tumor (C6, F98) glial cell lines were treated with 10ug transfected pI:C for 4 hours. At 24 hours post-transfection, cells were harvested and total RNA was extracted and used to quantify expression levels of several interferon stimulated genes including IRF-1, GBP-1, and Mx1/2. qRT-PCR was performed as described on the LightCycler 480 (Molecular Resource Center, UTHSC) using Universal Primary Library (UPL) protocol for rat gene expression (Roche) (See Chapter 2 for description of primer sets). Levels of expression were compared to those induced in FR rat fibroblasts.

Results were normalized to the housekeeping gene, Cyclophilin B. From these results, we were able to confirm that the designed primer sets worked in these cell models and that non-tumor cells demonstrate higher levels of expression for each of the ISGs tested over glioma cell lines. 


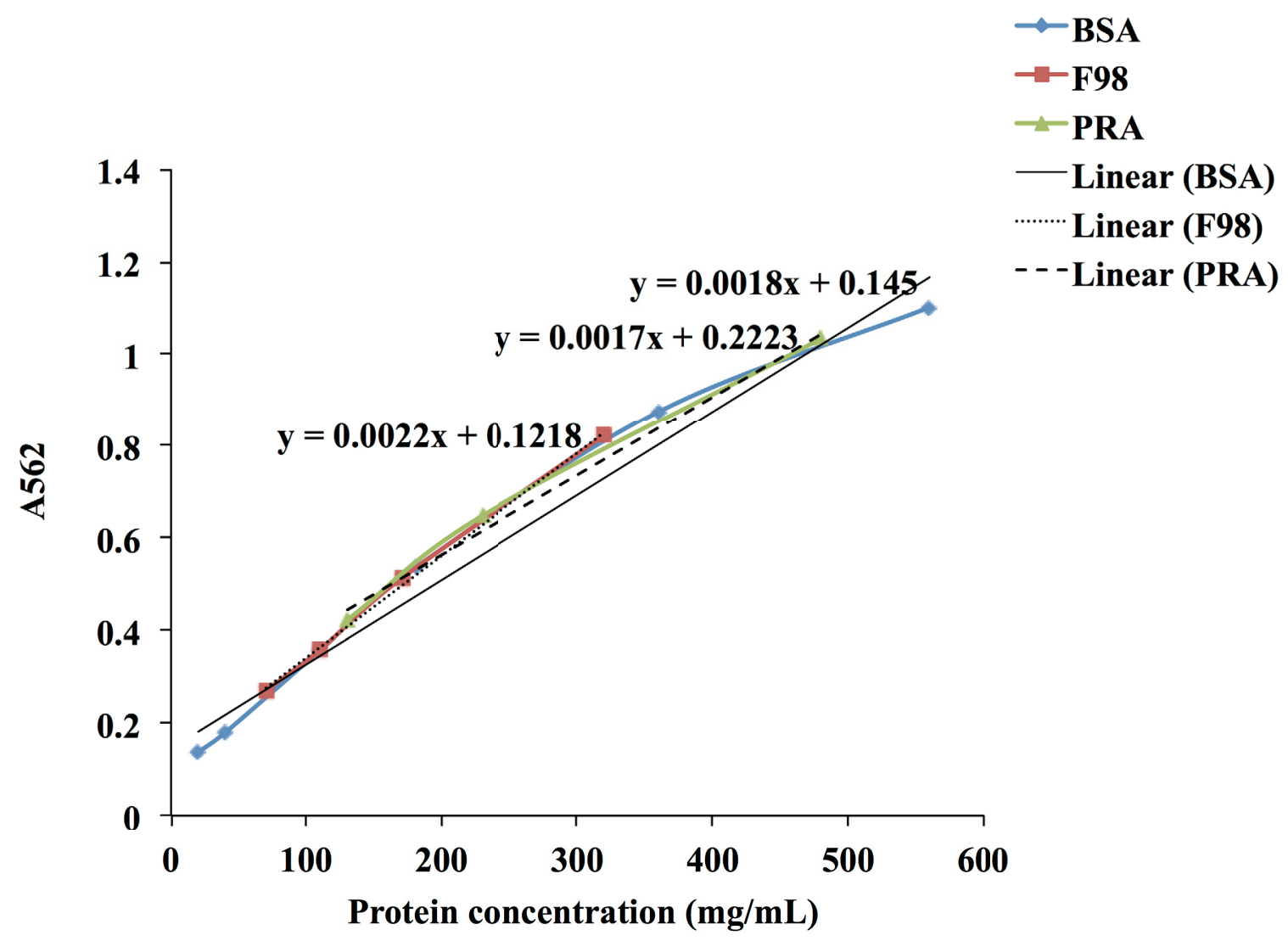

Figure A-4. BCA protein assay of proteins derived from glial cell lysate In order to determine the concentration of proteins derived from cell lysate of several glial cell lines, a BCA colorimetric assay was performed according to manufacturer instructions (BCA Protein Assay Reagent, Pierce). Cells were lysed in RIPA buffer (25mM Tris- $\mathrm{HCl}, 0.1 \%$ SDS, $1 \%$ Triton X-100, 1\% sodium deoxycholate, $0.15 \mathrm{M} \mathrm{NaCl}$, $1 \mathrm{mM}$ EDTA) and measurements were taken by spectrophotometer at $\mathrm{A}_{562}$. 


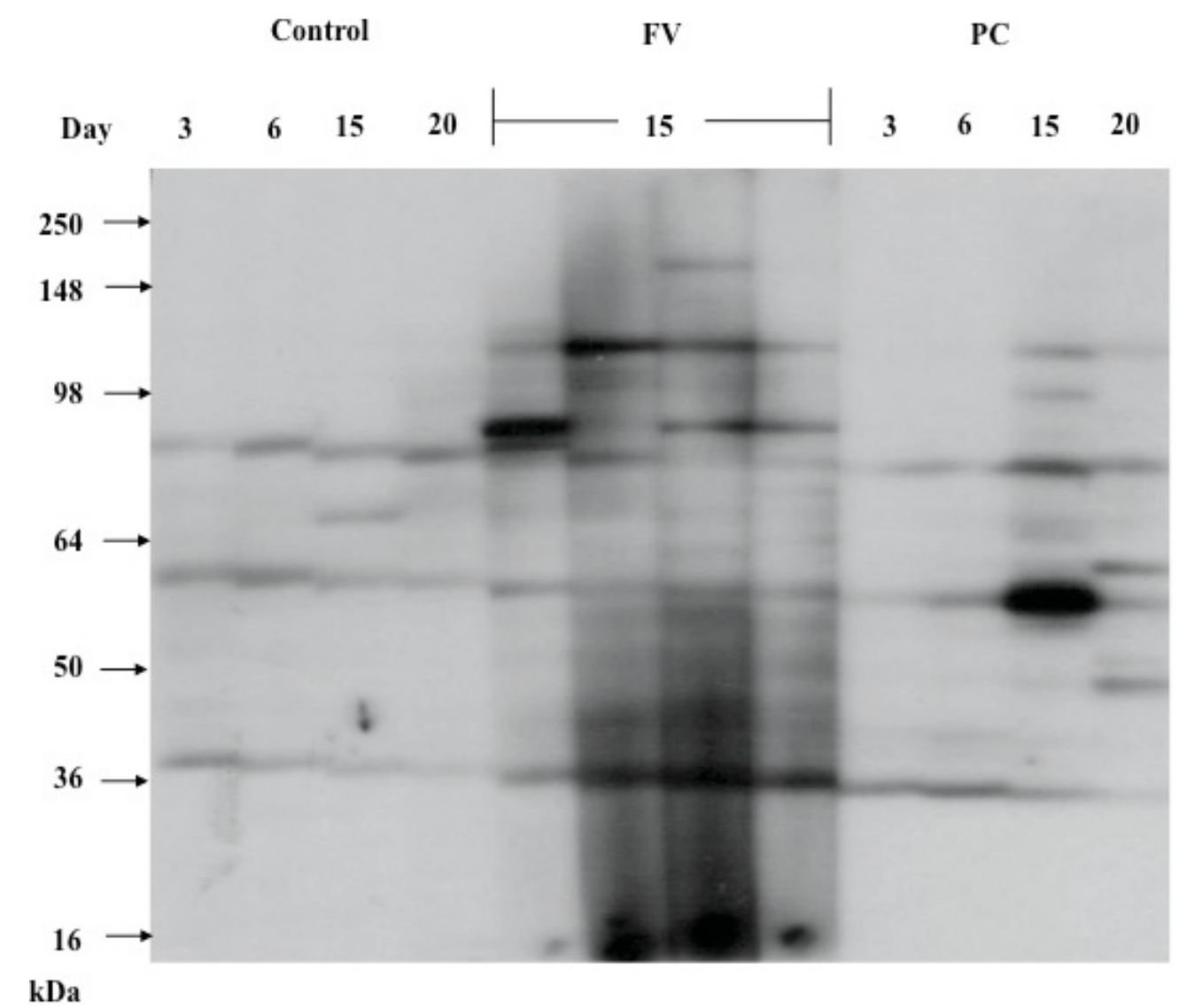

Figure A-5. Detection of circulating anti-tumor antibodies in animals treated with pre-infected cells versus free virus

Sera from intracranial F98 glioma bearing animals that were not treated or treated with either pre-infected (PC) F98 tumor cells or Free Virus (FV) were collected at the time of euthanasia as described. Cultured F98 cells from a 10 cm dish were harvested and lysed in $1 \mathrm{~mL}$ RIPA buffer. Protein concentrations were determined using the BCA colorimetric method against known concentrations of BSA (Pierce, Rockford, IL) as described. For SDS-PAGE, lysates were suspended in Laemmli reducing sample buffer and heated in $100{ }^{\circ} \mathrm{C}$ sand bath for 5 minutes. Samples were then centrifuged at $15,000 \mathrm{xg}$ for one minute and left on the bench to come to room temperature. Ten microliters of SeeBlue Plus2 protein standard (Invitrogen) were loaded next to $10 \mu \mathrm{g}$ of lysate and resolved on $0.75 \mathrm{~mm} \mathrm{9 \%} \mathrm{SDSPAGE} \mathrm{gel.} \mathrm{Gels} \mathrm{were} \mathrm{transferred} \mathrm{to} \mathrm{PVDF}$ membrane which were then blocked in 5\% NFDM/TTBS at room temperature for one hour. Membranes were then cut and incubated at room temperature for two hours in sera at 1:500 in 5\% NFDM/TTBS. Membranes were washed in TTBS and then incubated at room temperature for two hours in goat $\alpha$-rat secondary antibody conjugated to HRP (Jackson Immunoresearch Laboratories) at a dilution of 1:5000. Signal was visualized using chemiluminescence (SuperSignal West Dura, Pierce) followed by exposure to and development of X-ray film 


\section{APPENDIX B. STANDARDIZING THE IFN BIOASSAY AFTER VSV INFECTION}

\section{Introduction and Purpose}

A number of studies have evaluated IFN activity following infection with wtVSV as well as with a number of VSV matrix (M) mutants that are thought to be interferon inducers [276, 289, 291, 292]. Very often these studies determine IFN activity based on levels of gene expression using semi-quantitative or quantitative RT-PCR [289, 371] or by quantifying total IFN protein using methods such as ELISA [193, 289]. As we have seen, the induction of IFN gene expression does not necessarily correlate with the amount of biologically active IFN produced. In fact, from our results, the production of active IFN appears to be highly regulated in non-tumor cells such as primary astrocytes regardless of the high levels of induced IFN gene expression. In order to test for IFN activity, we have used an IFN bioassay in which IFN in a sample is determined by its ability to inhibit VSV-induced CPE [367]. In samples already containing infectious virus, the IFN bioassay is impossible to interpret. Various neutralizing techniques have been used in other studies such as acid treatment [290] or heat inactivation [344], however, results do not take into account the effects these methods potentially have on the stability of IFN. In the following studies, we evaluated several neutralizing methods including acid treatment, heat neutralization, and ultracentrifugation. The following results show the effectiveness of these methods by determining the amount of residual infectious particles post-treatment and by testing the effect of these treatments on IFN activity.

\section{Materials and Methods}

\section{Cells}

L929 murine fibroblasts were provided by Dr. Lawrence Pfeffer. BHK21 cells were supplied by Dr. Michael Whitt.

\section{Neutralization Methods}

Based on the high viral progeny produced by F98 glioma cells during rNCP12.1 infection in vitro $\left(10^{7}-10^{9} \mathrm{pfu} / \mathrm{mL}\right)$, we tested several methods of neutralization against samples containing high titers of virus, specifically $10^{8} \mathrm{pfu}$ wtVSV. For each technique, residual virus was detected by standard plaque assay on BHKs. Effects on IFN activity were determined by comparing 200U of treated IFN with non-treated rIFN using the IFN bioassay in L929 murine fibroblasts. rIFN units were previously determined with respect to the international reference standard for mouse interferon alpha/beta (mouse IFN- $\alpha / \beta$ (NIH). 


\section{Neutralization by anti-VSV neutralizing antibody (TN-1)}

TN-1 is a polyclonal antibody developed in rabbits immunized with intact UVinactivated wtVSV. In previous studies, $10 \mu \mathrm{L}$ of TN-1 neutralized $10^{5} \mathrm{pfu}$ wtVSV (data not shown). For these studies, $20 \mu \mathrm{L}$ of TN-1 was incubated at $37^{\circ} \mathrm{C}$ for one hour with $10^{8}$ pfu wtVSV. Residual virus was detected using a standard plaque assay on BHKs.

\section{Heat inactivation} [344].

Virus and rIFN were both heated separately in a $56^{\circ} \mathrm{C}$ water bath for 30 minutes

\section{Acid neutralization}

Virus and rIFN were treated with $50 \mu \mathrm{L}$ of $0.5 \mathrm{M} \mathrm{HCl}(\mathrm{pH}=2)$ and incubated for $2 \mathrm{~h}$ at $4^{\circ} \mathrm{C}$ then neutralized by adding $45-50 \mu \mathrm{L}$ of $0.5 \mathrm{M} \mathrm{NaOH}[290]$.

\section{Combination of heat inactivation and acid neutralization}

We evaluated combination treatment of heat $\left(56^{\circ} \mathrm{C}\right)$ and acid neutralization $(\mathrm{pH}$ of 2 ) on wtVSV and IFN activity. Samples were first heated for 30 minutes then treated with acid for 2 hours at $4^{\circ} \mathrm{C}$.

\section{$20 \%$ sucrose cushion}

$10^{8} \mathrm{pfu}$ wtVSV was suspended in $2 \mathrm{~mL}$ of serum free DMEM, over-layed onto a $20 \%$ sucrose cushion, and centrifuged for 35 minutes at 45,000 rpm using an AH650 rotor at $4^{\circ} \mathrm{C}$. $200 \mathrm{U}$ IFN diluted into $2 \mathrm{~mL}$ SF-DMEM was also spun through a $20 \%$ sucrose cushion in the same manner.

\section{Combination of $20 \%$ sucrose cushion and acid neutralization}

$10^{8}$ pfu virus was treated with $0.5 \mathrm{M} \mathrm{HCl}$ at a pH of 2 for 2 hours at $4^{\circ} \mathrm{C}$ then brought back to neutral $\mathrm{pH}$ using $\mathrm{NaOH}$. Acid treated samples were then spun through a $20 \%$ sucrose cushion as described.

\section{Ultra centrifugal filtration}

Virus was suspended in $1 \mathrm{~mL}$ of SF DMEM. Samples were centrifuged in Amicon Millipore Ultracel Filters with a pore cutoff 100,000 NMWL. This cutoff would 
ensure that viral particles would be separated from IFN in the media based on the inability of intact virus particles to move through the membrane pores. IFN, on the other hand, having a size of approximately $20 \mathrm{kDa}$, would be able to pass freely. Samples were spun at $5000 \mathrm{rpm}$ for 10 minutes at $4^{\circ} \mathrm{C}$ using JS 4.2 rotor. Following one spin, filtered supernatant was titered using a standard plaque assay on BHKs. Five hundred microliters of IFN at a concentration of $1 \mathrm{U} / \mu \mathrm{L}$ was also filtered as described.

\section{Results}

Following treatment with $\mathrm{TN}-1$, no residual virus was detected by plaque assay at 24hpi however between 36-48 hours, multiple plaques began to form. Heat inactivation resulted in no remaining infectious virions for one sample and $2 \mathrm{pfu} / \mathrm{mL}$ in another, however, IFN activity was decreased 4 fold relative to the untreated IFN standard. Plaque analysis of acid treated virus resulted in a titer of $3.1 \times 10^{3} \mathrm{pfu} / \mathrm{mL}$. A subsequent study using acid neutralization however resulted in no detectable infectious virus. Results showed that acid decreased IFN activity 2 fold. A combination of heat and acid treatment neutralized all virus however IFN activity was decreased 4 fold relative to the IFN standard in one sample and was undetectable following heat and acid treatment in another sample. A titer of $1.6 \times 10^{2} \mathrm{pfu} / \mathrm{mL}$ was detected following centrifugation through a $20 \%$ sucrose cushion and IFN activity was shown to be 2 fold lower relative to untreated rIFN $\beta$. A combination of acid with $20 \%$ sucrose cushion was able to remove and inactivate all virus from one sample with $40 \mathrm{pfu} / \mathrm{mL}$ virus remaining in another. However IFN activity was decreased 4 fold. Lastly, following one spin using the ultracentrifugal filters, supernatants were found to have $26 \mathrm{pfu} / \mathrm{mL}$ of virus remaining however a second spin resulted in no residual virus. IFN activity was found to be unaffected by this technique after one or two cycles of filtration.

\section{Discussion}

Because the IFN bioassay requires that L929 cells be treated for 24 hours in neutralized supernatant followed by another 24 hours to score inhibition of viral induced $\mathrm{CPE}$, the likelihood that results would be contaminated by non- neutralized virus in samples treated with neutralizing antibody would be high. Therefore, this strategy would not be appropriate for our purposes. Heat, acid, and sucrose treatments all diminished IFN activity between two and four-fold with one sample having undetectable IFN activity. In addition, inactivation of infectious virus was inconsistent as some samples had no detectable virus while others demonstrated infectious viral particles as seen by plaque assay following treatments. Therefore, these treatments would not be appropriate as well for our studies. Ultra-centrifugal filtration was found to be the most appropriate, effective, and reliable method. Though one spin allowed some virus to filter likely through defects in the membrane, a second spin was able to separate all viral particles without any reduction in IFN activity. Therefore, we concluded that two spins using the Amicon Millipore filters at 5000rpm for 10 minutes each would be used for subsequent studies. 


\section{VITA}

Erika Alicia Dillard-Cannon was born in 1979 in Memphis, Tennessee. She graduated from S.R. Butler High School in Huntsville, Alabama in 1997 and, later, from Vanderbilt University in Nashville, Tennessee in 2001 with a Bachelor of Science in Neuroscience. After graduation, she worked in several labs at Vanderbilt University, the University of Memphis, and the University of Tennessee Health Science Center (UTHSC) on projects ranging from neurobiology to pharmacology. Erika then enrolled in the College of Medicine with the entering class of 2004 at UTHSC and, in 2006, matriculated into the $\mathrm{MD} / \mathrm{PhD}$ dual degree program in the College of Graduate Health Sciences at UTHSC where she joined the Integrated Program of Biomedical Sciences (IPBS) in the Cancer and Developmental Biology track. Under the guidance of Dr. Michael A. Whitt (Microbiology, Immunology, and Biochemistry) Erika has worked on developing an oncolytic virus for the treatment of glioblastoma multiforme.

Erika will graduate from the $\mathrm{MD} / \mathrm{PhD}$ dual degree program in December 2013 and will be pursuing a career in neurosurgery with a research focus in experimental therapies for malignant brain tumors. 